\title{
Digitale Bildanalyse zur Messung fraktaler Eigenschaften der Bodenstruktur
}

\author{
Dissertation \\ zur Erlangung des Doktorgrades \\ der Mathematisch-Naturwissenschaftlichen Fakultäten \\ der Georg-August-Universität zu Göttingen
}

vorgelegt von

Diplom-Geoökologin Annette Dathe

aus Braunschweig

Göttingen 2001 
D 7

Referent: $\quad$ Prof. Dr. Gerhard Gerold

Korreferent: PD Dr. Jürgen Niemeyer

Tag der mündlichen Prüfung: 27.06.2001 


\section{Vorwort}

Die vorliegende Dissertation entstand im Rahmen des SFB 468 „Wechselwirkungen an Geologischen Grenzflächen“ an der Fakultät für Geowissenschaften und Geographie der Universität Göttingen. Seit dem 01.10.00 erfolgte die Förderung seitens der DFG im Rahmen einer Sachbeihilfe unter der Fördernummer GE 431/14-1. Der Deutschen Forschungsgemeinschaft sei für die finanzielle Unterstützung gedankt, sowie der Universität Göttingen, die in der Überganszeit die Finanzierung sicherte.

Herrn Prof. G. Gerold sei gedankt für die Übernahme des Referats, die Überlassung des Themas und die allgemeine Unterstützung, auf die jederzeit zurückgegriffen werden konnte. Ohne seine großzügige Gewährung wissenschaftlichen Freiraums wäre diese Arbeit nicht in der vorliegenden Form möglich gewesen. Herrn PD Dr. J. Niemeyer möchte ich für die Übernahme des Korreferats und viele interessante Diskussionen danken und für seinen Elan, die Arbeit voranzutreiben.

Mein besonderer Dank gilt Herrn Dr. S. Eins, der mich in die Arbeitsweisen der digitalen Bildanalyse und in die Wissenschaft der fraktalen Geometrie einführte. Die in dieser Untersuchung verwendeten Messprogramme sind seiner „Feder“ zu verdanken und er hat das Projekt auch im Ruhestand weiterhin gewissenhaft betreut.

In der Anfangszeit des Teilprojekts A6 „Grenzflächenreaktivität: Aufbau und Zerfall von Mikroaggregaten“ standen mir Frau N. Walter und Herr O. Friedrichs zur Seite. Frau Walter unterstützte mich intensiv und unermüdlich bei der Erstellung der Aufnahmen am Rasterelektronenmikroskop und den mittels digitaler Bildanalyse durchzuführenden Messungen. Herr Friedrichs führte den Großteil der bodenphysikalischen Untersuchungen mit enormer Selbstständigkeit und großer Wissbegierigkeit durch. Beide waren immer zu fruchtbaren Diskussionen bereit. Frau C. Burkard sei gedankt für die Präparation der Dünnschliffe und die Erstellung vieler lichtmikroskopischer Aufnahmen. Herr Ph. Burgess und Herr M. Tienken übernahmen Teile der umfangreichen Datenverarbeitung, wofür ihnen herzlich gedankt sei.

Frau M. Müller und die Herren F. Stange, J. Klingauf, J. Sutmöller, S. Eins und U. Birkel übernahmen das Korrekturlesen, entdeckten die ein oder andere Unstimmigkeit und brachten viele Sachverhalte klarer zum Ausdruck. Ihnen allen sei herzlich gedankt.

Herrn A. Ringeler möchte ich für das Bereitstellen des $\mathrm{C}^{++}$Codes für das robuste Schätzverfahren mittels lokalen M-Schätzern danken.

Bedanken möchte ich mich auch bei allen Kolleginnen und Kollegen, die wissentlich und unwissentlich zu dieser Arbeit beigetragen haben, indem sie Tips und Anregungen gaben oder mich in interessante Dispute verwickelten. 



\section{Inhaltsverzeichnis}

Vorwort............................................................................................................................ I

Verzeichnis der Abkürzungen und Symbole.......................................................................IV

Verzeichnis der Abbildungen ..............................................................................................VI

Verzeichnis der Tabellen ................................................................................................ X

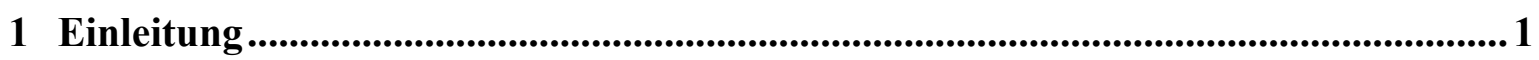

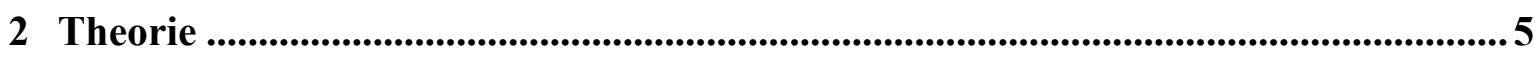

2.1 Theorie der Fraktale ................................................................................

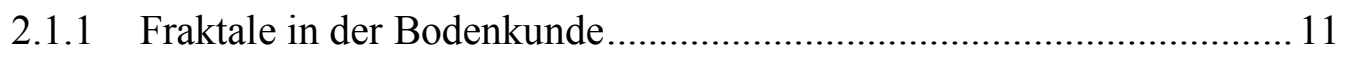

2.2 Bodenphysikalische Grundlagen ………………………………………..... 13

2.2.1 Parametrisierung von Retentions- und Leitfähigkeitskurven ................. 17

3 Material und Präparation .........................................................................................21

3.1 Herkunft des verwendeten Bodenmaterials ....................................................21

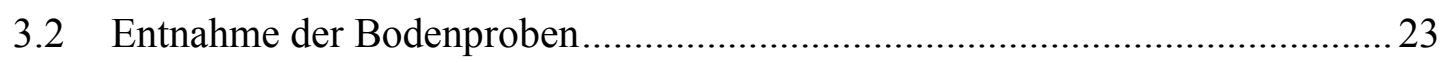

3.3 Präparation der Bodenproben für Dünnschliffe ............................................. 23

4 Methoden .................................................................................................................25

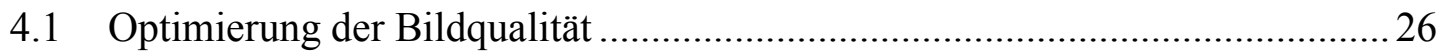

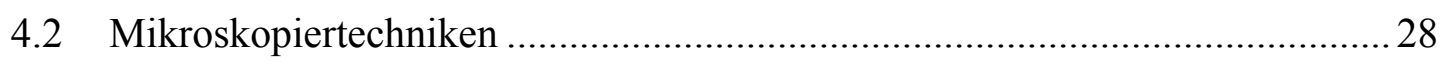

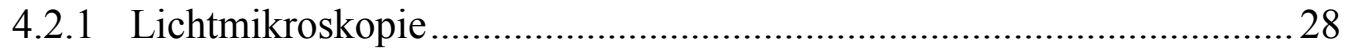

4.2.2 Feldemissions-Rasterelektronenmikroskopie......................................28

4.3 Anwendung der verschiedenen Mikroskopiertechniken....................................30

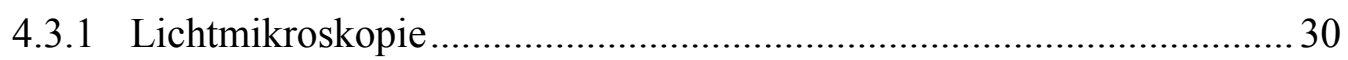

4.3.2 Feldemissions-Rasterelektronenmikroskopie...........................................33

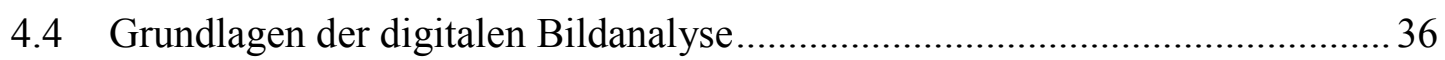

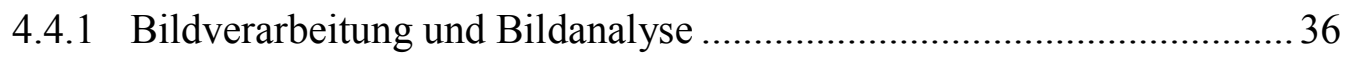

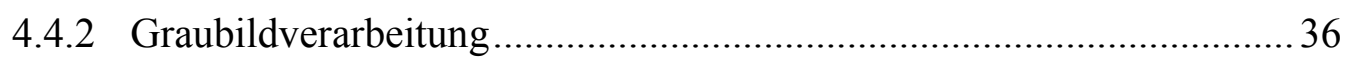

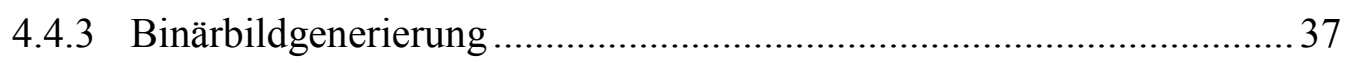

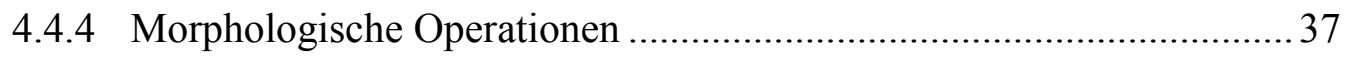

4.4.5 Objektmessung und stereologische Verfahren ............................................ 38

4.4.6 Erstellung von Anwendungsprogrammen für das KS400 .......................38

4.5 Anwendung der digitalen Bildverarbeitung ..................................................... 38

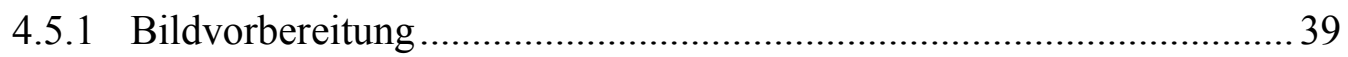

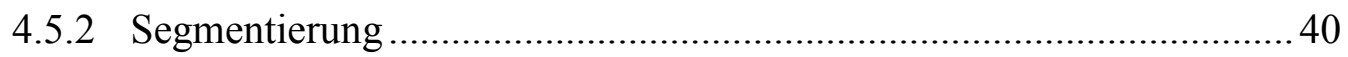

4.5.3 Bildnachbereitung.......................................................................... 40

4.6 Messung der fraktalen Dimension der Porenraumgrenzlinie............................. 41

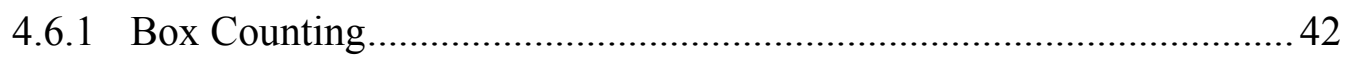

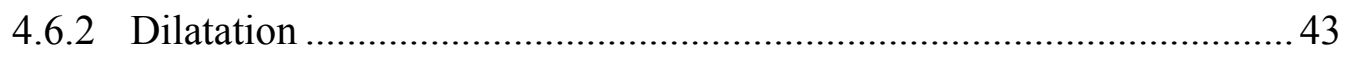


4.6.3 Untersuchung der Auswirkung verschiedener Auflösungen ................. 44

4.6.4 Messung der Porengrößenverteilung................................................. 44

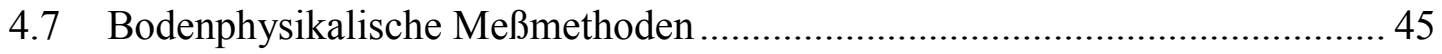

4.7.1 Bestimmung der Phasenanteile ......................................................... 46

4.7.2 Ermittlung von Retentionskurven ................................................. 46

4.7.3 Messung der gesättigten Wasserleitfähigkeit.................................... 46

4.7.4 Messung der ungesättigten Wasserleitfähigkeit ................................ 46

5 Mathematische Auswertung und Statistik ........................................................... 49

5.1 Berechnung der fraktalen Dimension durch lineare Regression .....................50

5.1.1 Bestimmung des verwendeten Datenbereichs (Cutoffs) ...................... 50

5.1.1.1 Bestimmung der Cutoffs für Boxcounting ..................................... 52

5.1.1.2 Bestimmung der Cutoffs für Dilatation .......................................... 53

5.1.2 Berechnung der Mittelwerte mit den Methoden „A“ und „B“.............. 54

5.1.3 Anpassung der Daten verschiedener Vergrößerungsstufen eines

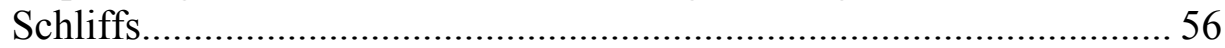

5.1.4 Lineare und exponentielle Schrittweiten ........................................... 57

5.1.5 Anpassung von Regressionsgeraden mit lokalen M-Schätzern ............ 57

5.2 Parameterschätzung für die Abhängigkeit der fraktalen Dimension von der

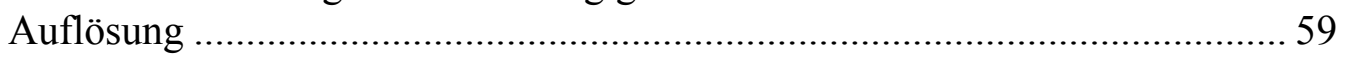

5.3 Berechnung der Porengrößenverteilung ..................................................... 59

5.3.1 Summierung der Porengrößenverteilung verschiedener Vergrößerungsstufen 60

5.4 Parametrisierung der im Labor ermittelten Retentions- und

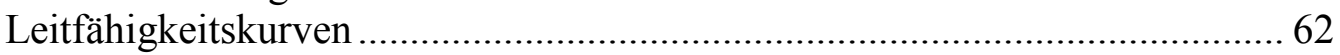

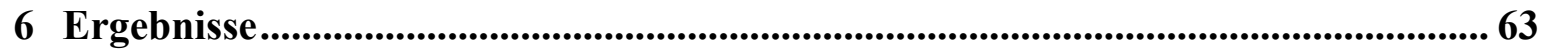

6.1 Die fraktale Dimension der Porenraumgrenzlinie .......................................... 63

6.1.1 Messungen an FE-REM Aufnahmen ................................................. 63

6.1.1.1 Abweichung der Steigung D von der Linearität................................ 67

6.1.1.2 Anpassung von Regressionsgeraden an alle Vergrößerungsstufen .... 69

6.1.2 Messungen an lichtmikroskopischen Aufnahmen................................ 73

6.1.2.1 Untersuchung der für die REM-Aufnahmen verwendeten Dünnschliffe75

6.1.2.2 Untersuchung von horizontal und vertikal ausgerichteten

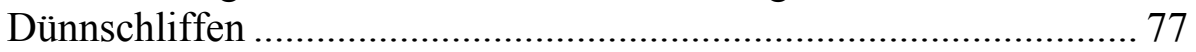

6.1.3 Abhängigkeit der fraktalen Dimension von der Bildauflösung............. 81

6.2 Abhängigkeit der fraktalen Dimension von der Auflösung bei verschiedenen

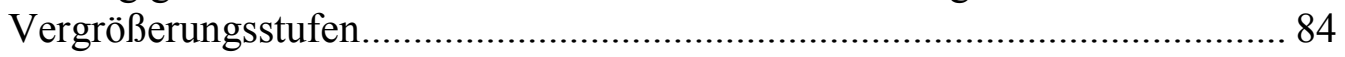

6.3 Ergebnisse der Messung der Porengrößenverteilung ..................................... 88

6.3.1 Messungen an FE-REM Aufnahmen ................................................ 89

6.3.2 Vergleich der kumulierten Porengrößenverteilung mit der Retentionskurve.

6.3.3 Darstellung der Porengrößenverteilung aller Vergrößerungsstufen...... 93

6.3.4 Messungen an lichtmikroskopischen Aufnahmen................................ 94

6.4 Die Steigung der Ableitung der Porengrößenverteilung ................................. 96 
6.5 Ergebnisse der bodenphysikalischen Untersuchungen 101

6.5.1 Bestimmung der Phasenanteile........................................................ 101

6.5.2 Messung der gesättigten und der ungesättigten Wasserleitfähigkeit... 102

6.5.3 Schätzung der Parameter für die Retentionskurve 103

6.5.4 Berechnung der ungesättigten Wasserleitfähigkeit und Vergleich mit den Messdaten

6.6 Vergleich zwischen den Parametern der Retentionskurve und den bildanalytisch gemessenen fraktalen Dimensionen .... 105

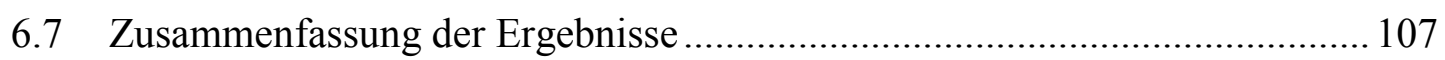

6.7.1 Die fraktale Dimension der Porenraumgrenzlinie ............................. 107

6.7.2 Die Messung der Porengrößenverteilung .......................................... 110

6.7.3 Vergleich mit der Parametrisierung der Retentionskurve ................... 110

7 Diskussion 113

7.1 Diskussion der verwendeten präparativen und mikroskopischen Methoden. 113

7.2 Diskussion der digitalen Bildanalyse. 114

7.3 Diskussion der verwendeten mathematischen und statistischen Methoden... 115

7.4 Diskussion der bodenphysikalischen Methoden ............................................. 116

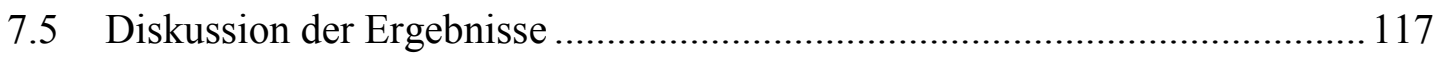

8 Zusammenfassung....................................................................................................... 121

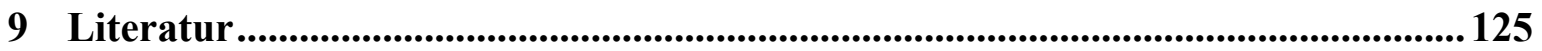

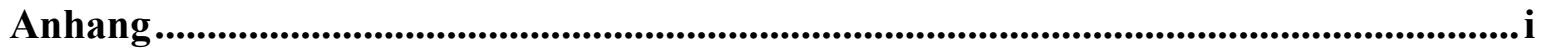

\section{Lebenslauf}




\section{Verzeichnis der Abkürzungen und Symbole}

$\mathrm{d}_{\mathrm{B}} \quad$ Lagerungsdichte des Bodens $\left[\mathrm{ML}^{-3}\right]$

$\mathrm{d}_{\mathrm{F}} \quad$ Dichte der Festsubstanz $\left[\mathrm{ML}^{-3}\right]$

$\mathrm{g} \quad$ Erdbeschleunigung $\left[9.81 \mathrm{~ms}^{-2}\right]$

$\mathrm{h}_{\mathrm{c}}$ : Steighöhe der Wassersäule in der Kapillare [L]

i Laufindex für Summen [1]

$\mathrm{n} \quad$ Laufindex der Iteration [1]

$\mathrm{n} \quad$ Anpassungsparameter der van Genuchten-Gleichung [1]

$\mathrm{m}$ Anpassungsparameter der van Genuchten-Gleichung [1]

q Flußdichte oder Filtergeschwindigkeit $\left[\mathrm{LT}^{-1}\right]$

$r_{c} \quad$ Kapillarenradius [L]

sd Standardabweichung

se Standardfehler

$\mathrm{t} \quad$ Zeit [T]

$\mathrm{x} \quad$ Richtungsvektor [L]

y Richtungsvektor [L]

z Richtungsvektor, Tiefe [L]

A Fläche $\left[\mathrm{L}^{2}\right]$

D fraktale Dimension

$\mathrm{D}^{\mathrm{b}} \quad$ Boxcounting-Dimension

$\mathrm{D}^{\mathrm{d}} \quad$ Dilatations-Dimension

$\mathrm{D}_{\mathrm{L}} \quad$ fraktale Dimension einer Länge

$D_{S} \quad$ fraktale Dimension einer Fläche

$\mathrm{D}_{\mathrm{H}}$ Hausdorff Dimension

$\mathrm{D}_{\mathrm{c}} \quad$ Kapillarenradius [L]

F Menge [1]

F Fläche $\left[\mathrm{L}^{2}\right]$

$\mathrm{H}^{\mathrm{s}} \quad$ allgemeines s-dimensionales Hausdorff $\mathrm{Ma} \beta$

$\mathrm{H}_{\varepsilon}^{\mathrm{s}} \quad$ s-dimensionales Hausdorff Maß

$\mathrm{K}$ Wasserleitfähigkeit [L/T, $\left.\mathrm{cm} \mathrm{d}^{-1}\right]$

$\mathrm{K}_{\mathrm{r}} \quad$ relative hydraulische Leitfähigkeit [1]

$\mathrm{K}_{\mathrm{s}}$ gesättigte hydraulische Leitfähigkeit $\left[\mathrm{LT}^{-1}, \mathrm{~cm} \mathrm{~d}^{-1}\right]$

L Länge [L]

$\mathrm{N} \quad$ Anzahl [1]

Q Volumenstrom pro Querschnittsfläche $\left[\mathrm{L}^{3} \mathrm{~L}^{-2} \mathrm{~T}^{-1}=\mathrm{LT}^{-1}\right]$

$\mathrm{U} \quad$ Umfang [L]

$\mathrm{V}$ n-dimensionales Volumen $\left[\mathrm{L}^{\mathrm{n}}\right]$

$V_{\mathrm{P}} \quad$ Porenvolumen $\left[\mathrm{L}^{3}\right]$

$\alpha \quad$ Anpassungsparameter der van Genuchten-Gleichung $\left[\mathrm{hPa}^{-1}\right]$

$\hat{\alpha} \quad$ Schätzer für den $\mathrm{y}$-Achsenabschnitt der linearen Regression

$\beta \quad$ Benetzungswinkel $\left[{ }^{\circ}\right]$

$\hat{\beta} \quad$ Schätzer für die Steigung der linearen Regression

$\varepsilon \quad$ Größe des skalierenden Elementes [L, $\mu \mathrm{m}]$ 
$\lambda$ Anpassungsparameter nach Brooks und Corey

$\psi \quad$ Gesamtpotential $\left[\mathrm{ML}^{-1} \mathrm{~T}^{-2}, \mathrm{hPa}\right]$

$\psi_{\mathrm{b}} \quad$ Lufteintrittspunktl $\left[\mathrm{ML}^{-1} \mathrm{~T}^{-2}, \mathrm{hPa}\right]$

$\psi_{\mathrm{g}} \quad$ Gaspotential $\left[\mathrm{ML}^{-1} \mathrm{~T}^{-2}, \mathrm{hPa}\right]$

$\psi_{\mathrm{H}}$ hydraulisches Potential $\left.\mathrm{ML}^{-1} \mathrm{~T}^{-2}, \mathrm{hPa}\right]$

$\psi_{\mathrm{m}} \quad$ Matrixpotential $\left[\mathrm{ML}^{-1} \mathrm{~T}^{-2}, \mathrm{hPa}\right]$

$\psi_{\mathrm{o}} \quad$ osmotisches Potential $\left[\mathrm{ML}^{-1} \mathrm{~T}^{-2}, \mathrm{hPa}\right]$

$\psi_{\mathrm{z}} \quad$ Gravitationspotential $\left[\mathrm{ML}^{-1} \mathrm{~T}^{-2}, \mathrm{hPa}\right]$

$\rho \quad$ Dichte der Flüssigkeit $\left[\mathrm{ML}^{-3}\right]$

$\sigma \quad$ Grenzflächenspannung $\left[\mathrm{MT}^{-2}\right]$

$\sigma^{2} \quad$ Varianz

$\theta \quad$ volumetrischer Wassergehalt $\left[\mathrm{L}^{3} \mathrm{~L}^{-3}\right]$

$\theta_{\mathrm{r}}$ residualer Wassergehalt $\left[\mathrm{L}^{3} \mathrm{~L}^{-3}\right]$

$\theta_{\mathrm{s}}$ gesättigter Wassergehalt $\left[\mathrm{L}^{3} \mathrm{~L}^{-3}\right]$

$\Theta \quad$ effektive Sättigung [1]

$\partial \quad$ partielle Ableitung

IR+ Menge der positiven reellen Zahlen 


\section{Verzeichnis der Abbildungen}

Abb. 2.1: Die ersten fünf Iterationsschritte der triadischen Kochkurve...................................................6

Abb. 2.2: Der vierte Iterationsschritt der Koch'schen Schneeflocke. ......................................................... 8

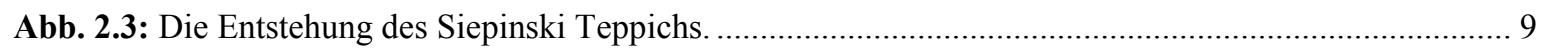

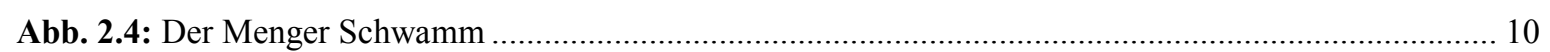

Abb. 2.5: Schematische Retentionskurven eines Sand-, Schluff- und Tonbodens .................................... 16

Abb. 3.1: Herkunft des verwendeten Bodenmaterials sudöstlich von Göttingen........................................ 22

Abb. 4.1: Fließdiagramm der vorbereitenden Arbeiten für die digitale Bildanalyse. ................................. 26

Abb. 4.2: Licht- und rasterelektronenmikroskopische Aufnahmen eines Bodendünnschliffs mit den entsprechenden Grauwertverteilungen. ............................................................................... 27

Abb. 4.3: Schematische Darstellung der Bestrahlung einer Probe mit dem Elektronenstrahl ...................... 29

Abb. 4.4: Lichtmikroskopische Aufnahme einer Tonablagerung (Zeiss Axiophot)................................... 30

Abb. 4.5: Aufnahmen am Leitz Orthoplan. (a) Im Durchlicht; (b) mit gekreuzten Polarisatoren; (c) zusätzlich mit Quarz Rot I Kompensator; (d) Binärbild von (b) . ........................................................... 31

Abb. 4.6: Durchlichtaufnahmen des Dünnschliffes Wild7 mit dem Wild-Photomakroskop.

(a) Im Hellfeld; (b) mit polarisiertem Licht; (c) Binärbild von (b) ............................................ 32

Abb. 4.7 a-c: Beispielhafte Darstellung von FE-REM Bildern der Serie Leo8.......................................... 34

Abb. 4.7 d-e: Beispielhafte Darstellung von FE-REM Bildern der Serie Leo8......................................... 35

Abb. 4.8: Ablaufschema der digitalen Bildverarbeitung und Bildanalyse................................................. 39

Abb. 4.9: Schematische Darstellung der Wahl der Segmentierungsschwelle............................................ 40

Abb. 4.10: Konturen der Grenzlinie Pore/Matrix über dem Originalbild ............................................... 41

Abb. 4.11: Beispiel (Momentaufnahme) für das Boxcounting-Verfahren.................................................. 42

Abb. 4.12: Dilatation am Beispiel des vernetzten Porensystems im Boden ............................................ 43

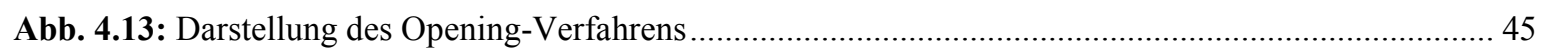

Abb. 4.14: Schematischer Aufbau der Ku-Apparatur zur Messung der ungesättigten Wasserleitfähigkeit. .. 47

Abb. 5.1: Methoden der Datenverarbeitung zur Bestimmung der fraktalen Dimension.............................. 49

Abb. 5.2 a-b: Richardson Plots für die in Abb. 4.2 d und e dargestellten REM Aufnahme.

(a) Alle Messwerte und (b) innerhalb der Cutoffs. ................................................................... 51

Abb. 5.2 c: Entsprechender Richardson Plot für das Dilatations-Verfahren............................................ 52

Abb. 5.3: Bestimmung des unteren Cutoffs für Boxcounting am Beispiel der Serie Leo8, für jeden Messschritt gemittelte Daten

Abb. 5.4: Bestimmung des unteren Cutoffs für Dilatation am Beispiel eines mit der feinsten Auflösung aufgenommenen Einzelbildes. 54

Abb. 5.5: Datenblatt für die lineare Regression nach Methode B (Mittelwerte einer Vergrößerungsstufe)... 55

Abb. 5.6: Ergebnisse des Boxcounting-Verfahrens für die einzelnen Vergrößerungsstufen innerhalb der unteren und oberen Cutoffs 56

Abb. 5.7: Anpassung einer Geraden für alle Vergrößerungsstufen des Schliffes Leo8. 57

Abb. 5.8: Absolute Abweichung für die sukzessive Anpassung von zwei Regressionsgeraden für das Boxcounting-Verfahren und den Schliff Leo8.

Abb. 5.9: Anpassung von zwei Geraden an die in Abb. 5.7 gezeigten Daten des Schliffes Leo8 59 
Abb. 5.10: Vergleich der Porengrößenverteilungen für Leo8 mit der Retentionskurve.

(a) Einzeln dargestellt; (b) aufsummiert und (c) Darstellung ohne die ersten fünf Messwerte bis zu der Größenklasse, die auf 50 \% aller Bilder vorkommt.

Abb. 6.1: Ergebnisse der Boxcounting-Methode für die verschiedenen Auflösungsstufen innerhalb des unteren und oberen Cutoffs des Schliffes Leo7 (Methode B)..

Abb. 6.2: Ergebnisse der Boxcounting Methode für die verschiedenen Auflösungsstufen innerhalb des unteren und oberen Cutoff des Schliffes Leo9 (Methode B).

Abb. 6.3: Besonders ausgeprägte Abweichung der Messpunkte von der Regressionsgeraden. (a) Linearer Abstand der Schrittweiten und (b) mit exponentieller Schrittweite.

Abb. 6.4: REM-Aufnahme mit deutlich unterschiedlichen Porengrößen. Die Grenzlinie ist mit einem Pixel Breite schwarz markiert

Abb. 6.5: Alle Vergrößerungsstufen des Schliffes Leo8, nach der Methode B gemittelte und bezüglich des yAchsenabschnitts angepasste Messdaten der Dilatations-Methode .....

Abb. 6.6: Anpassung von zwei Geraden an die in Abb. 6.5 gezeigten Daten des Schliffes Leo8.

Abb. 6.7: Anpassung von zwei Geraden an die Boxcounting-Verfahren gewonnenen Daten für das Einzelbild des für die Serie Leo8 verwendeten Dünnschliffs

Abb. 6.8: Die gleiche Aufnahme wie für Abb. 6.7, jedoch mit Dilatation gemessen.

Abb. 6.9: Anpassung von zwei Geraden an die Boxcounting-Daten für die LM-Aufnahmen der vertikal ausgerichteten Dünnschliffe einer Kubiena-Box

Abb. 6.10: Die gleichen Aufnahmen wie für Abb. 6.9, gemessen mit Dilatation. 78

Abb. 6.11: Anpassung von zwei Geraden an die Boxcounting-Daten für die LM-Aufnahmen der horizontal ausgerichteten Dünnschliffe einer Kubiena-Box.....

Abb. 6.12: Die gleichen Aufnahmen wie für Abb. 6.11, gemessen mit Dilatation.

Abb. 6.13: REM-Aufnahme der 500x Vergrößerungsstufe, die mit unterschiedlichen Auflösungen gespeichert wurde.

Abb. 6.14: Der mittlere Cutoff am Beispiel von Leo8, dargestellt über der Bildauflösung sowie die mit linearer Regression geschätzten mittleren Cutoffs (s. Tab. 6.13).

Abb. 6.15: Abhängigkeit der gemessenen fraktalen Dimension von der Auflösung.

(a) Für Leo7, Boxcounting; (b) für Leo8, Boxcounting und (c) für alle drei Messserien und Boxcounting und Dilatation.

Abb. 6.16: Ergebnisbild des Opening-Verfahrens über dem Originalbild.

Abb. 6.17 a-c: Distributive Porengrößenverteilung der Serie Leo7 (siehe Tab A1) .................................. 90

Abb. 6.17 d-e: Fts.

Abb. 6.18: Vergleich der Porengrößenverteilungen für Leo7 mit der am selben Bodenhorizont aufgenommenen Retentionskurve.

Abb. 6.19: Vergleich der Porengrößenverteilungen für Leo8 mit der am selben Bodenhorizont aufgenommenen Retentionskurve.

Abb. 6.20: Klassennormierte Porengrößenverteilung aller Vergrößerungsstufen für den Dünnschliff Leo7. 93

Abb. 6.21: Klassennormierte Porengrößenverteilung aller Vergrößerungsstufen für den Dünnschliff Leo8. 94

Abb. 6.22: Distributive Porengrößenverteilung der Serie WK2P hor (Wild Klotz 2, horizontale Ausrichtung).

Abb. 6.23: Distributive Porengrößenverteilung der Serie WK2P ver (Wild Klotz 2, vertikale Ausrichtung). 95

Abb. 6.24: Anpassung von einer Geraden mit dem robusten Schätzverfahren an die logarithmisch dargestellte distributive Porengrößenverteilung von Leo7. 
Abb. 6.25: Anpassung von einer Geraden mit dem robusten Schätzverfahren an die logarithmisch dargestellte distributive Porengrößenverteilung von Leo8

Abb. 6.26: Anpassung von zwei Geraden an die logarithmisch dargestellte distributive Porengrößenverteilung von Leo7. (a) Die absoluten Abweichungen zeigen zwei Minima. (b) Anpassung von zwei Geraden für das erste Minimum und (c) für das zweite Minimum.

Abb. 6.27: Anpassung von zwei Geraden an die logarithmisch dargestellte distributive Porengrößenverteilung von Leo8. (a) Die absoluten Abweichungen zeigen zwei Minima. (b) Anpassung von zwei Geraden für das erste Minimum und (c) für das zweite Minimum..... 99

Abb. 6.28: Ungesättigte Wasserleitfähigkeiten von 8 an der Ku-Apparatur gemessenen Parallelproben .... 102

Abb. 6.29: Im Labor gemessene Werte von Wassergehalt und Wasserspannung und die Anpassung von Retentionskurven nach van Genuchten unter der Annahme eines uni- und eines bimodalen Porensystems. Anpassungsparameter s. Tab. 6.18.

Abb. 6.30: Im Labor gemessene Werte von Wassergehalt und Wasserspannung und die Anpassung einer Retentionskurve nach Brooks und Corey unter der Annahme eines unimodalen Porensystems. Anpassungsparameter s. Tab. 6.18.

Abb. 6.31: Berechnung der ungesättigten Wasserleitfähigkeit nach Mualem und van Genuchten unter der Annahme eines uni- und eines bimodalen Porensystems. Zum Vergleich sind die gemessenen ungesättigten Wasserleitfähigkeiten dargestellt.

Abb. 6.32: Schematische Darstellung von gemessenen fraktalen Dimensionen für drei typische Partikelformen. 


\section{Abbildungen im Anhang}

Abb. A1: Durchlichtaufnahmen des Dünnschliffes Wild 8 (horizontale Ausrichtung) mit dem Photomakroskop (Wild), Objektiv 6.3. (a) im Hellfeld, (b) mit polarisiertem Licht, (c) nach Grauwertsegmentierung erhaltenes Binärbild von (b).

Abb. A2: Durchlichtaufnahmen des Dünnschliffes Wild 9 (horizontale Ausrichtung) mit dem Photomakroskop (Wild), Objektiv 6.3. (a) im Hellfeld, (b) mit polarisiertem Licht, (c) nach Grauwertsegmentierung erhaltenes Binärbild von (b).

Abb. A.3-1: Ergebnisse des robusten Schätzverfahrens für alle Vergrößerungsstufen des Schliffes Leo7.

(a) Anpassung einer Geraden für Boxcounting; (b) Absolute Abweichungen für die Anpassung von zwei Geraden; (c) Anpassung von zwei Geraden

Abb. A.3-2: Ergebnisse des robusten Schätzverfahrens (lokale M-Schätzer) für alle Vergrößerungsstufen des Schliffes Leo7. (d) Anpassung einer Geraden für die nach der Methode B gemittelten Daten des Dilatationsverfahrens und (e) Anpassung von zwei Geraden für die Daten von (d).... iv

Abb. A.4-1: Ergebnisse des robusten Schätzverfahrens (lokale M-Schätzer) für alle Vergrößerungsstufen des Schliffes Leo9. (a) Anpassung einer Regressionsgeraden für die nach der Methode B gemittelten Daten des Boxcounting-Verfahrens; (b) die gleichen Daten wie in (a), die Anpassung von zwei Geraden erfolgte für die geringste absolute Abweichung; (c) Anpassung einer Geraden für die nach der Methode B gemittelten Daten des Dilatationsverfahrens; ....

Abb. A.4-2: Ergebnisse des robusten Schätzverfahrens (lokale M-Schätzer) für alle Vergrößerungsstufen des Schliffes Leo9. (d) Darstellung der absoluten Abweichungen für die Anpassung von zwei Geraden für Dilatation und (e) Anpassung von zwei Geraden für die Daten von (c), die Optimierung erfolgte durch die Minimierung der absoluten Abweichung.

Abb. A.5: Alle Vergrößerungsstufen des Schliffes Leo7 unter Verwendung einer exponentiellen Schrittweite.

(a) Anpassung von zwei Geraden für Boxcounting und (b) für Dilatation.. vii

Abb. A.6: Alle Vergrößerungsstufen des Schliffes Leo8 unter Verwendung einer exponentiellen Schrittweite.

(a) Anpassung von zwei Geraden für Boxcounting und (b) für Dilatation.. viii

Abb. A.7: Alle Vergrößerungsstufen des Schliffes Leo9 unter Verwendung einer exponentiellen Schrittweite.

(a) Anpassung von zwei Geraden für Boxcounting und (b) für Dilatation..

Abb. A.8: Anpassung von zwei Geraden (lokale M-Schätzer) für die LM-Aufnahme Wild7. (a) Mit Boxcounting und (b) mit Dilatation erhobene Messdaten.

Abb. A.9: Anpassung von zwei Geraden (lokale M-Schätzer) für die LM-Aufnahme Wild9. (a) Mit Boxcounting und (b) mit Dilatation erhobene Messdaten. xi

Abb. A.10: Abhängigkeit der gemessenen fraktalen Dimension von der Auflösung (s. Tab. 6.14).

(a) Darstellung für Leo7, Dilatation und (b) für Leo8, Dilatation. xii

Abb. A.11: Abhängigkeit der gemessenen fraktalen Dimension von der Auflösung (s. Tab. 6.14).

(a) Graphische Darstellung für Leo9, Boxcounting und (b) für Leo9, Dilatation...... .xiii

Abb. A.12: Distributive Porengrößenverteilung der Serie Leo8 (s. Tab. A2).. xiv

Abb. A.13: Vergleich der Porengrößenverteilungen für Leo7 mit der Retentionskurve. (a) Einzeln dargestellt; (b) aufsummiert und (c) Darstellung ohne die ersten fünf Messwerte bis zu der Größenklasse, die auf $50 \%$ aller Bilder vorkommt.. 


\section{Verzeichnis der Tabellen}

Tab. 2.1: Korngrößenfraktionen nach der deutschen Nomenklatur.

Tab. 2.2: Einteilung der Porengrößenbereiche nach dem Porendurchmesser und der korrelierenden Wasserspannung.....

Tab. 3.1: Feldanspracheergebnisse und bodenchemische Merkmale der untersuchten Parabraunerde......... 21

Tab. 3.2: Im Labor ermittelte bodenphysikalische Parameter der Parabraunerde (Profil Waldschlößchen).. 23

Tab. 4.1: Vergrößerungsstufen, Auflösungen und Bildgrößen der verschiedenen Mikroskopietechniken. ... 33

Tab. 6.1: Fraktale Dimensionen für jede einzelne Vergrößerungsstufe der Messserie Leo7 und für deren Berechnung relevante Parameter

Tab. 6.2: Fraktale Dimensionen für jede einzelne Vergrößerungsstufe der Messserie Leo 8 und für deren Berechnung relevante Parameter

Tab. 6.3: Fraktale Dimensionen für jede einzelne Vergrößerungsstufe der Messserie Leo9 und für deren Berechnung relevante Parameter.

Tab. 6.4: Fraktale Dimensionen der Serie Leo7, vertikale Schliffausrichtung. Die Berechnung erfolgte über alle Vergrößerungsstufen.

Tab. 6.5: Fraktale Dimensionen der Serie Leo8, horizontale Schliffausrichtung. Die Berechnung erfolgte über alle Vergrößerungsstufen.

Tab. 6.6: Fraktale Dimensionen der Serie Leo9, horizontale Schliffausrichtung. Die Berechnung erfolgte über alle Vergrößerungsstufen.

Tab. 6.7: Fraktale Dimensionen der für die Leo-Serien 7, 8 und 9 benutzten Schliffe, gemessen an mit dem Wild-Photomakroskop aufgenommenen Einzelbildern. Die Anpassung von zwei Geraden erfolgte mit einer Optimierung durch lokale M-Schätzer .

Tab. 6.8: Fraktale Dimensionen der für die Leo-Serien 7, 8 und 9 benutzten Schliffe, gemessen an mit dem Wild-Photomakroskop aufgenommenen Einzelbildern. Die Anpassung durch Minimierung der Abweichungsquadrate erfolgte für einen optimierten Bereich.

Tab. 6.9: Fraktale Dimensionen der Serie Wild Klotz 2, gemessen mit Boxcounting und Dilatation. Die Berechnung erfolgte differenziert in horizontale und vertikale Schliffausrichtungen mit einer Optimierung durch lokale M-Schätzer .....

Tab. 6.10: Fraktale Dimensionen und für deren Berechnung relevante Parameter der Serie Wild Klotz 2. Die Berechnung erfolgte differenziert in horizontale und vertikale Schliffausrichtungen nach der Methode der kleinsten Abweichungsquadrate.

Tab. 6.11: Fraktale Dimensionen und für deren Berechnung relevante Parameter eines mit 150x Vergrößerung und verschiedenen Auflösungen am FE-REM aufgenommenen Einzelbildes der Serie Leo8.

Tab. 6.12: Wie Tab. 6.10 für ein mit 500x Vergrößerung und verschiedenen Auflösungen am FE-REM aufgenommenes Einzelbild der Serie Leo8.

Tab. 6.13: Zusammenhang zwischen dem mittleren logarithmischen Cutoff (y) und der für die jeweilige Vergrößerungsstufe verwendeten Auflösung (x).

Tab. 6.14: Mit dem Levenberg-Marquard-Verfahren und einer Optimierung durch Minimierung der Abweichungsquadrate geschätzte Parameter für die minimale $\left(\mathrm{D}_{\text {Smin }}\right)$ und die maximale $\left(\mathrm{D}_{\text {Smax }}\right)$ fraktale Dimension sowie den Anpassungsparameter k. ...

Tab. 6.15: Nach den Gl. 6.3 und 6.4 berechnete optimale Auflösung $\varepsilon_{\text {opt }}$ und der kritische Cutoff, bei dem die maximale fraktale Dimension $\mathrm{D}_{\max }$ erreicht ist.....

Tab. 6.16: Steigungen der logarithmierten distributiven Porengrößenverteilung der Serien Leo7 und Leo8100 
Tab 6.17: Ergebnisse der Korngrößenanalyse (s. Tab. 2.1) und der Bestimmung der Phasenanteile. 101

Tab. 6.18: Anpassungsparameter zur Beschreibung der Retentionskurve unter der Annahme eines uni- und eines bimodalen Porensystems 103

Tab. 6.19: Aus den Parametrisierungen der Retentionskurve berechnete fraktale Dimensionen. 106

\section{Tabellen im Anhang}

Tab. A.1: Auflösungen und Reichweiten bzw. Porengrößen für die Messung der Porengrößenverteilung am Schliff Leo7.. xvii

Tab. A.2: Auflösungen und Reichweiten bzw. Porengrößen für die Messung der Porengrößenverteilung am Schliff Leo8. xix 



\section{$1 \quad$ Einleitung}

Im Boden durchdringen sich Lithosphäre, Hydrosphäre, Atmosphäre und Biosphäre und formen ein Gebilde, das an Komplexität kaum zu übertreffen ist, die Pedosphäre. Im Kleinen betrachtet besteht ein Boden aus Mineralkörnern unterschiedlicher Art und Größe und der organischen Substanz, dem Humus. Wenngleich für die Bodengenese von großer Bedeutung, soll hier nicht weiter auf die organische Substanz eingegangen werden. Die Mineralkörner formen ein Gefüge im Raum, welches von einem Hohlraumsystem, den Poren, durchzogen ist.

Die Poren im Boden können mit Wasser oder Bodenluft gefüllt sein. Sie dienen als Transportsystem für Wasser und darin gelöste oder mit dem Wasser konvektiv mitgeführte Stoffe. Die Belüftung des Bodens findet ebenfalls durch das Porensystem statt und damit der Transport gasförmiger Stoffe. Die Untersuchung der Bodenstruktur hat eine lange Tradition, wobei sie sich der direkten Betrachtung entzieht. Zur Charakterisierung des Wasserhaltevermögens eines Bodenhorizonts und um den zur Lösung der Wassertransportgleichung benötigten Zusammenhang zwischen Unterdruck und Wassergehalt zu erlangen, werden Retentionskurven erstellt.

Dies erfolgt üblicherweise an Stechzylinderproben im Labor, kann aber auch aus im Gelände erhobenen Daten abgeleitet werden, wenn Wassergehalt und Matrixpotential (welches die Bindung des Bodenwassers an die Matrix beschreibt) zeitgleich erfasst werden. Das Matrixpotential (oder, mit positivem Vorzeichen, die Wasserspannung) verhält sich umgekehrt proportional zum Porenradius, der bei diesem bestimmten Unterdruck entwässern würde. So wird häufig aus dem Volumen des bei einer definierten Saugspannung ausgetriebenen Wassers auf die Verteilung der Poren bestimmter Größenklassen im Boden geschlossen. Dabei geht die Struktur des Bodens nur indirekt über die Erfassung der hydraulisch wirksamen Kapillaren ein.

Die fraktale Dimension (D) beschreibt das Skalierungsverhalten eines selbstähnlichen Musters, das sich über mehrere Größenordnungen wiederholt. Die Kennzahl D beschreibt das Verhältnis zwischen einem grundlegenden Muster oder Generator und einer Iterationsregel. Viele in der Natur vorkommende Systeme können so beschrieben werden, wie z. B. Flusseinzugssysteme, Bäume und die Verästelungen des Kapillarsystems im Blutkreislauf. Die fraktale Dimension einer Struktur kann gemessen werden, wobei die digitale Bildanalyse ein machtvolles Werkzeug darstellt, das die schnelle Verarbeitung großer Datenmengen erlaubt (Eins 1995). Der erhaltene Wert für D drückt die Komplexität einer Struktur aus und liefert somit ein quantitatives Ergebnis im Gegensatz zu einer qualitativen Beschreibung.

In der vorliegenden Arbeit erfolgt die direkte Erfassung der inneren Geometrie des Bodens mit der Methode der digitalen Bildanalyse. Als Untersuchungsgegenstand dienen Dünnschliffpräparate des Tonanreicherungshorizonts einer Parabraunerde aus Löss. Gemessen werden die Porengrößenverteilung und die fraktale Dimension der Grenzfläche zwischen Poren und Matrix. Die Untersuchungen werden an digitalen Bildern durchgeführt, die für Übersichtsaufnahmen mit einem Polarisations-Mikroskop im Durchlicht und für detaillierte Untersuchungen mit verschiedenen Vergrößerungsstufen eines Feldemmissions-Rasterelektronenmikroskops erstellt werden. 
Der Grenzfläche zwischen Pore und Matrix kommt für die Charakterisierung des Porenraums eine besondere Bedeutung zu. Dies gilt sowohl für die hydraulischen Eigenschaften des porösen Mediums als auch für die Beschreibung der inneren Oberfläche des Porensystems, das sich über mehrere Größenordnungen erstreckt. Nach Perrier et al. (1999) besteht ein direkter Zusammenhang zwischen dem Massenfraktal der Bodenpartikel, dem Porenfraktal und dem Fraktal der Grenzfläche zwischen beiden Phasen im Boden. In diesem Sinne stellt die bildanalytische Messung der Porengrößenverteilung, die hier analog zur Retentionskurve aufgetragen wird, auch eine Messung des Porenfraktals dar.

Für die Messung der fraktalen Dimension der Porenraumgrenzlinie werden die Methoden Boxcounting und Dilatation benutzt. Das Boxcounting-Verfahren legt ein Gitter von Kästchen mit wachsender Kantenlänge über das Bild und zählt jeweils die Kästchen, die die untersuchte Struktur beinhalten. Beim Dilatations-Verfahren wird die Grenzlinie dilatiert und jeweils die Länge der Umhüllenden in Abhängigkeit ihres Durchmessers gemessen. Wird die Messgröße über dem Durchmesser des Strukturelements aufgetragen, ergibt sich in logarithmischer Auftragung der Theorie entsprechend eine Gerade, deren Steigung die fraktale Dimension darstellt.

Für eine nachvollziehbare Berechnung von D aus den Rohdaten ist große Sorgfalt nötig. Natürliche Objekte weisen eine Grenze der Selbstähnlichkeit auf, z. B. im Größenbereich von Molekülen. Für kleine Strukturelemente weist der log-log-Plot eine systematische Abweichung von einer Geraden auf. So ist die Entscheidung wichtig, welche Datenpaare zur Berechnung der Steigung beitragen, im Größenbereich weniger Pixel führt bereits das Weglassen eines einzigen Datenpaares zu signifikanten Änderungen für $\mathrm{D}$ im Größenbereich der ersten Nachkommastelle. Die als Entscheidungskriterium benutzten Methoden werden vorgestellt. Es werden zwei verschiedene Methoden zur Mittelwertbildung pro untersuchtem Dünnschliff und Vergrößerungsstufe verwendet, (A) der Mittelwert der für einzelne Bilder ermittelten Steigungen und (B) die Mittelung der Rohdaten, woraus die Berechnung der Steigung erfolgt.

Die Untersuchung der mit dem Polarisations-Mikroskop erstellten Übersichtsaufnahmen von benachbarten, horizontal und vertikal ausgerichteten Schliffen erfolgt in Hinblick auf eine mögliche Differenz der gemessenen D für verschieden Schliffrichtungen.

Parallel zu den bildanalytischen Methoden werden an direkt benachbart entnommenen Proben desselben Bodenhorizontes klassische bodenphysikalische Untersuchungen durchgeführt. Neben einer Korngrößenanalyse wird aus der Lagerungsdichte und der Dichte der Festsubstanz das Gesamtporenvolumen bestimmt. Die Erfassung von Retentions- und Wasserleitfähigkeitskurven an Stechzylinderproben im Labor erfolgt mit mehreren Parallelen. Die gesättigte Wasserleitfähigkeit wird an vertikal und horizontal entnommenen Stechzylindern gemessen. Die Parametrisierung der Retentionskurve erfolgt nach den gängigen Verfahren von Brooks \& Corey (1964) und van Genuchten (1980). Für die Parameter der van Genuchten-Gleichung wird nach Mualem (1976) die relative Wasserleitfähigkeit berechnet und mit den Messwerten verglichen. Die Anpassungsparameter werden in Hinblick auf fraktale Dimensionen diskutiert und mit den bildanalytisch gemessenen Porengrößenverteilungen in Beziehung gebracht. 
In der vorliegenden Arbeit kommen Methoden aus unterschiedlichen Fachgebieten zum Einsatz. Daher erfolgt im theoretischen Teil zunächst eine Beschreibung des Konzepts der fraktalen Geometrie, dann wird auf ihren Einsatz in der Bodenkunde eingegangen. Es folgen die klassischen bodenphysikalischen Grundlagen einschließlich ausgewählter Gleichungen zur Parametrisierung von Retentions- und Leitfähigkeitskurven. In den darauf folgenden Kapiteln wird das untersuchte Bodenmaterial genauer charakterisiert und die verwendeten Methoden werden beschrieben. Den mathematischen und statistischen Methoden ist ein eigenes Kapitel gewidmet. Die Darstellung der Ergebnisse und ihre Diskussion schließen sich an. 



\section{Theorie}

\subsection{Theorie der Fraktale}

Fraktale Geometrien stellen den Schlüssel zur Beschreibung selbstähnlicher Strukturen dar. Es handelt sich um Strukturen, deren Muster sich über mehrere Größenordnungen wiederholt, das heißt, unabhängig vom Abbildungsmaßstab (Vergrößerung oder Verkleinerung) zeigt sich im Idealfall immer dasselbe Muster. Beispiele in der Natur sind Blumenkohlröschen, Farnblätter, verzweigte Nervenzellfortsätze, Kapillarsysteme von Blutgefäßen, Küstenlinien (aber nicht in jedem Fall) und Ländergrenzen, weil sie häufig geografischen Grenzlinien wie Flußläufen und Gebirgskämmen folgen. Die Brownsche Molekularbewegung kann ebenso durch Fraktale beschrieben werden wie Zeitreihen von Börsenkursen. Seit den grundlegenden Arbeiten von Mandelbrot (1967) sind Fraktale (von lat. fractus $=$ gebrochen) sehr populär geworden. Es zeigt sich, dass fraktale Geometrien in der Natur die Regel und nicht die Ausnahme darstellen. Viele Wachstumsprozesse vor allem in der Biologie finden nach einem festgelegten Schema statt. Neuesten Kenntnissen zufolge (Sernetz 2000) liegt der Grund in dem geringen Speicherbedarf, den diese Art von Struktur auf dem genetischen Code benötigt. Im Grunde werden nur zwei Angaben gebraucht: Die Länge bzw. Form der Grundeinheit und eine Regel, die ihre Iteration beschreibt. Dadurch entstehen äußerst komplizierte Gebilde, die z. B. Kapillarsysteme dazu befähigen, durch feine Verästelungen eine sehr hohe Austauschrate zwischen zwei Systemen mit begrenzten Volumina zu erreichen, wobei der Materialverbrauch minimal bleibt. Selbstähnliche Muster in der unbelebten Natur bilden sich häufig durch konkurrierende Prozesse wie Diffusion und Aggregation (DLA $=$ Diffusion Limited Aggregation, Witten \& Sander 1981).

Bereits zu Beginn des 20. Jahrhunderts entdeckte die Mathematik die ungeheure Komplexizität geometrischer Gebilde, die durch fortlaufende Iteration aus einfachen Grundformen hervorgehen können. Beispiele sind das Cantor Set von Georg Cantor, die Kochkurve und Schneeflocke des schwedischen Mathematikers Helge von Koch und Sierpinskis Dreieck und Teppich. Die mathematischen Fraktale weisen eine Reihe erstaunlicher Eigenschaften auf, weshalb sie auch als mathematische Monster bezeichnet werden.

Um das Konzept von Fraktalen zu verdeutlichen, wird angenommen, N sei eine Anzahl von Volumenelementen (Intervalle, Quadrate, Würfel, Würfel höherer Ordnung der Größe $\varepsilon$ oder Intervalle, Kreise, Kugeln, Kugeln höherer Ordnung der Größe $\varepsilon$ ), die benötigt wird, um eine Menge $\mathrm{F}$ zu bedecken. Für kleine $\varepsilon$ entspricht die Summe der Volumenelemente dem Volumen V (F), wobei D die Dimension des Volumenelementes darstellt.

$$
\mathrm{V}(\mathrm{F})=\varepsilon^{\mathrm{D}} \cdot \mathrm{N}(\varepsilon)
$$

Intuitiv lässt sich für die Messung einer Länge, Fläche oder eines Volumens folgern, dass eine Dimension $\mathrm{D}=1,2$ oder 3 die Gl. 2.1 erfüllt. Tatsächlich kann gezeigt werden, dass dies auch für $\mathrm{D} \in \mathrm{IR}^{+}$gilt. Aus G1. 2.1 erhält man (Comegna et al. 1998) 


$$
\mathrm{D}=\frac{\log \mathrm{V}}{\log \varepsilon}-\frac{\log \mathrm{N}(\varepsilon)}{\log \varepsilon}
$$

Für sehr kleine Volumenelemente $(\varepsilon \rightarrow 0)$ konvergiert der erste Term $(\log V / \log \varepsilon)$ gegen 0 und man erhält für die fraktale Dimension D

$$
\mathrm{D}=\lim _{\varepsilon \rightarrow 0} \frac{\log \mathrm{V}}{\log \varepsilon}-\frac{\log \mathrm{N}(\varepsilon)}{\log \varepsilon}=-\lim _{\varepsilon \rightarrow 0} \frac{\log \mathrm{N}(\varepsilon)}{\log \varepsilon}
$$

Dies soll am Beispiel der triadischen Kochkurve gezeigt werden.

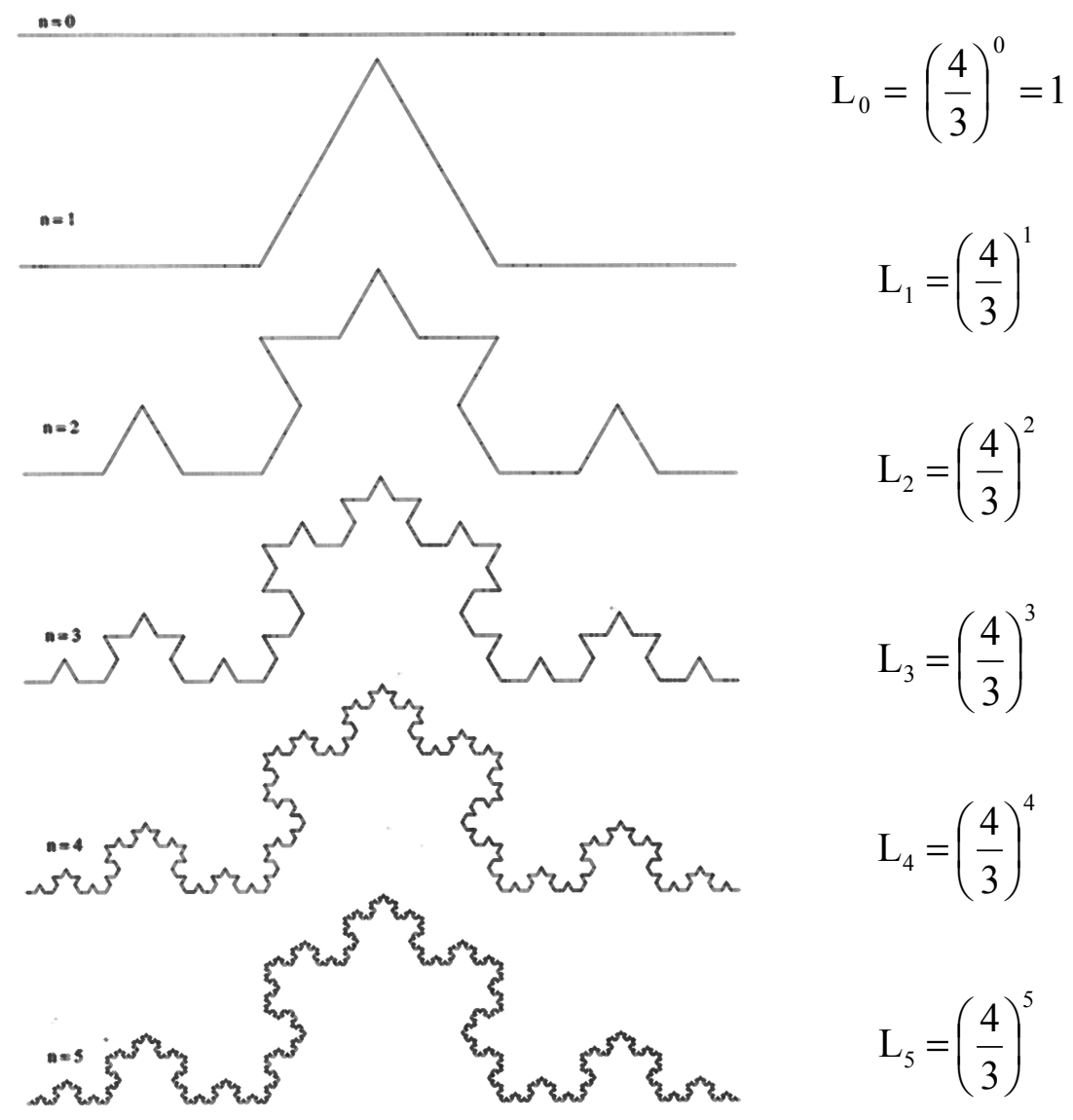

Abb. 2.1: Die ersten fünf Iterationsschritte der triadischen Kochkurve. Auf den Initiator wird ein Generator angewendet, der aus 4 Teilstrichen der Länge $\varepsilon$ (1/3 der Länge des Generators) besteht. Dieser Vorgang wird iteriert, indem der Generator fortlaufend auf jeden Teilstrich der vorangegangenen Iteration angewendet wird. Aus Feder 1988, verändert.

Sie besteht aus einem Initiator der Länge 1 und dem Generator $(n=1)$, der den Initiator in 4 Teilstriche der Länge $\varepsilon=1 / 3$ teilt. Jeder Teilstrich wird nach demselben Muster erneut in 4 Teilstriche zerlegt, so dass für $n=2$ die Kurve aus $4^{2}$ Teilstrichen der Länge $(\varepsilon=1 / 3)^{2}$ besteht. Wiederum wird auf jeden Teilstrich der herunterskalierte Generator angewendet. Die Länge L der Kochkurve für den n-ten Schritt errechnet sich nach der Formel 


$$
\mathrm{L}(\varepsilon)=4^{\mathrm{n}} \cdot \varepsilon^{\mathrm{n}}=\left(\frac{4}{3}\right)^{\mathrm{n}} .
$$

Die Anzahl der Teilstrecken beträgt demnach $N=4^{\mathrm{n}}$

und die fraktale Dimension errechnet sich für $\varepsilon \rightarrow 0$ bzw. $n \rightarrow \infty$ nach

$$
\mathrm{D}=-\lim _{\varepsilon \rightarrow 0}\left(\frac{\log 4^{\mathrm{n}}}{\log \left(\frac{1}{3}\right)^{\mathrm{n}}}\right)=\frac{\log 4}{\log 3} \approx 1,2619 .
$$

Hier ist $1<\mathrm{D}<2$, da die fraktale Dimension einer Kurve (topologische Dimension 1) in einer Fläche (topologische Dimension 2) errechnet wurde. Die Länge der Kochkurve kann nach Gleichung 2.1 aus der Anzahl der Teilstriche $\mathrm{N}$ und der bei der n-ten Iteration aktuellen Länge eines Teilstrichs potenziert mit D bestimmt werden. Mit $\varepsilon=3^{-\mathrm{n}}$ kann der Laufindex der Iteration als

$$
\mathrm{n}=\frac{-\log \varepsilon}{\log 3}
$$

geschrieben werden (s. Feder 1988, S. 17). Eingesetzt in Gleichung 2.4 ergibt sich

$$
\begin{aligned}
\mathrm{L}(\varepsilon)=\left(\frac{4}{3}\right)^{\mathrm{n}}=10^{\left(\frac{-\log \varepsilon[\log 4-\log 3]}{\log 3}\right)} & =\varepsilon^{-(\log 4-\log 3) / \log 3} \\
& =\varepsilon^{1-(\log 4 / \log 3)}=\varepsilon^{1-\mathrm{D}}
\end{aligned}
$$

was einem Potenzgesetz zwischen der Länge des gesamten Objekts und der Länge des Segments $\varepsilon$ entspricht. Die Anzahl $N_{n}$ der Segmente $\varepsilon_{n}$ läßt sich ebenfalls als Potenzfunktion darstellen (Baveye \& Boast 1998). Aus $L(n)=N_{n} \varepsilon_{n}$ folgt

$$
\mathrm{N}=\varepsilon^{-\log 4 / \log 3}=\varepsilon^{-\mathrm{D}}
$$

Auf die Bedeutung der Gleichungen 2.8 und 2.9 wird in dem Kapitel 4.5.2 - Messung der fraktalen Dimension der Porenraumgrenzlinie - genauer eingegangen.

Die Gleichung 2.6 hat die allgemeine Form

$$
\mathrm{D}_{\mathrm{H}}:=\lim _{\varepsilon \rightarrow 0} \frac{\log \mathrm{N}}{\log (1 / \varepsilon)}
$$

Die Hausdorff Dimension $\mathrm{D}_{\mathrm{H}}$ ist nach dem deutschen Mathematiker Felix Hausdorff (1868-1942) benannt. Sie ist genau der kritische Exponent, an dem das Produkt $\mathrm{L}=\varepsilon^{\mathrm{D}_{\mathrm{H}}} \cdot \mathrm{N}$ gegen einen stabilen Wert konvergiert. Für Exponenten kleiner als $\mathrm{D}_{H}$ divergiert das Produkt gegen Unendlich, für Exponenten größer als $\mathrm{D}_{\mathrm{H}}$ konvergiert das 
Produkt gegen Null (Feder 1988, Schroeder 1991). Für $\varepsilon>0$ lautet die allgemeine Definition für das s-dimensionalen Hausdorff-Maß $\mathrm{H}_{\varepsilon}^{\mathrm{s}}$ (Falconer 1990, S. 25):

$$
\mathrm{H}_{\varepsilon}^{\mathrm{s}}(\mathrm{F})=\inf \left\{\sum_{\mathrm{i}=1}^{\infty}\left|\mathrm{U}_{\mathrm{i}}\right|^{\mathrm{s}}:\left\{\mathrm{U}_{\mathrm{i}}\right\} \text { ist eine } \varepsilon \text { - Bedeckung von } \mathrm{F}\right\} \text {. }
$$

Dabei bedeutet inf das infinum, die größte untere Grenze einer Menge. In Gleichung 2.11 betrachten wir die $\varepsilon$-Bedeckung von F und versuchen, die Summe der s-ten Potenz der Durchmesser $U_{i} \mathrm{zu}$ minimieren. Dieser Sachverhalt lässt sich noch allgemeiner ausdrücken,

$$
\mathrm{H}^{\mathrm{s}}(\mathrm{F}):=\lim _{\varepsilon \rightarrow 0} \mathrm{H}_{\varepsilon}^{\mathrm{s}}(\mathrm{F})
$$

was bedeutet, $\mathrm{H}^{\mathrm{s}}$ ist das s-dimensionale Hausdorff $\mathrm{Ma} ß$ der Menge $\mathrm{F}$, wobei $\mathrm{F}$ in jede euklidsche Dimension eingebettet sein kann.

Formen die mit einer fraktalen Dimension skalieren weisen eine Reihe erstaunlicher Eigenschaften auf. Als Beispiel sei hier die Koch'sche Schneeflocke dargestellt (s. Abb. 2.2).

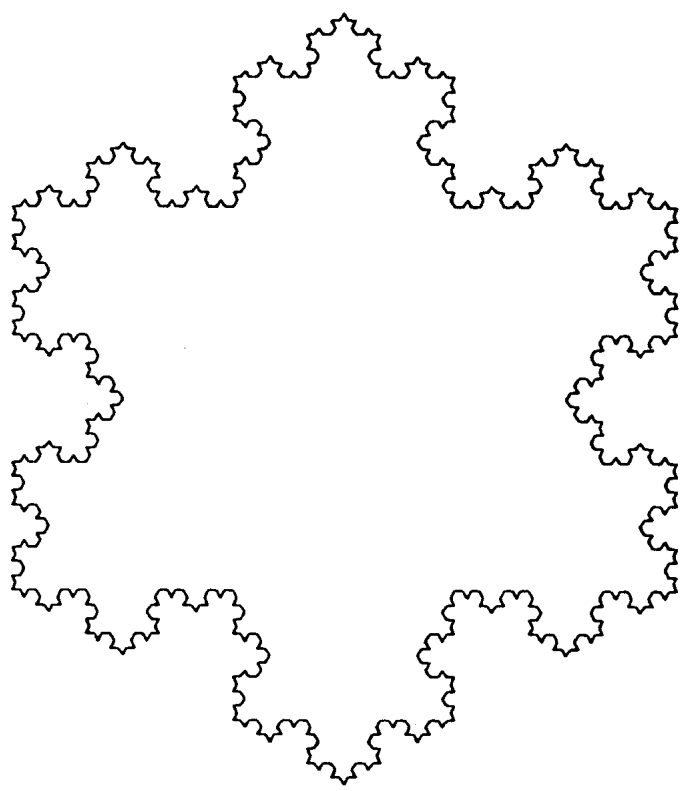

Abb. 2.2: Der vierte Iterationsschritt der Koch'schen Schneeflocke. Sie entsteht, wenn der in Abb. 2.1 gezeigte Iterator auf jede Seite eines gleichschenkligen Dreiecks angewendet wird.

Sie entsteht, wenn die triadische Kochkurve auf ein gleichseitiges Dreieck angewendet wird. Die fraktale Dimension ihrer Kontur entspricht dem der triadischen Kochkurve. Die Koch'sche Schneeflocke besitzt die erstaunliche Eigenschaft, dass ihr Umfang U mit jedem Iterationsschritt $\mathrm{n}$ zunimmt, es gilt

$$
\mathrm{U}_{\mathrm{n}}=\left(\frac{4}{3}\right)^{\mathrm{n}} \mathrm{U}_{0} \quad \text { und somit } \quad \lim _{\mathrm{n} \rightarrow \infty} \mathrm{U}_{\mathrm{n}}=\infty
$$


Die eingeschlossene Fläche $F_{n}$ dagegen läuft gemäß der geometrischen Reihe (Meyberg \& Vachenauer, 1993)

$$
\mathrm{F}_{\mathrm{n}}=\frac{\sqrt{3}}{4}+\sum_{\mathrm{i}=0}^{\mathrm{n}-1}\left(4^{\mathrm{i}} \cdot 3 \cdot\left(\frac{1}{9}\right)^{\mathrm{i}+1} \frac{\sqrt{3}}{4}\right)=\frac{\sqrt{3}}{4}\left(1+\frac{1}{3} \sum_{\mathrm{i}=0}^{\mathrm{n}-1}\left(\frac{4}{9}\right)^{\mathrm{i}}\right)
$$

gegen den Grenzwert

$$
\lim _{n \rightarrow \infty} F_{n}=\frac{\sqrt{3}}{4}\left(1+\frac{1}{3} \cdot \frac{9}{9-4}\right)=\frac{2}{5} \sqrt{3}
$$

Weitere, in der Bodenkunde beliebte Beispiele sind der Sierpinski Teppich (in der zweidimensionalen Fläche, s. Abb. 2.3) und der Menger Schwamm (im dreidimensionalen Raum, s. Abb. 2.4).
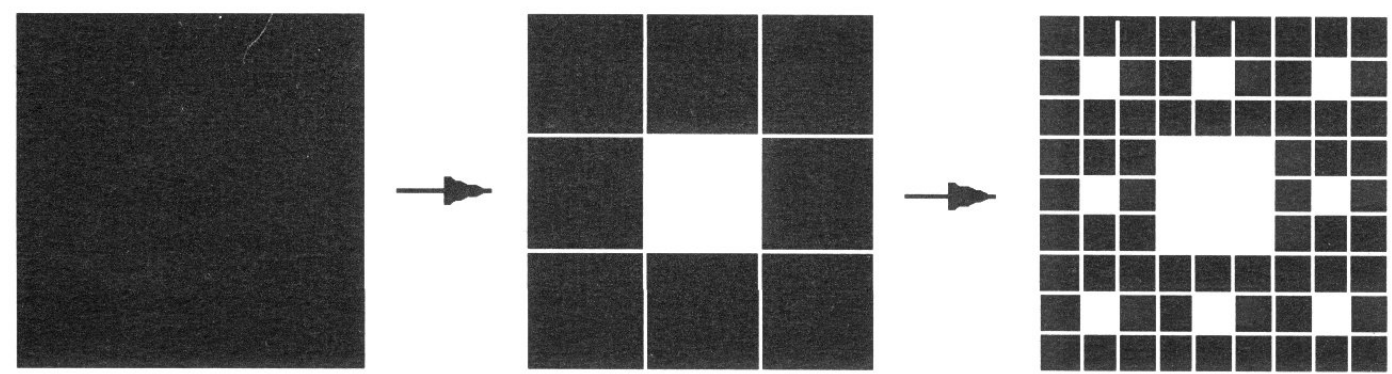

Abb. 2.3: Der Siepinski Teppich entsteht aus einem Quadrat, welches in $3 \times 3=9$ kleinere Quadrate unterteilt wird. Das mittlere Quadrat bleibt frei. Dieser Vorgang wird wiederholt. Aus Gouyet 1996, S. 17.

Für den Sierpinski-Teppich ist der Skalierungsfaktor 3 und das Massenverhältnis 8:1, die fraktale Dimension ergibt sich nach Gleichung 2.10 als

$$
\mathrm{D}=\frac{\log 8}{\log 3} \approx 1,893
$$

Der Menger Schwamm wird nach demselben Muster als Würfel gebildet, wobei beim ersten Iterationsschritt von jeder Seite aus bis zur gegenüberliegenden Seite die mittleren Quadrate herausgenommen werden. Die fraktale Dimension beträgt

$$
\mathrm{D}=\frac{\log 20}{\log 3} \approx 2,727
$$

d. h., beim i-ten Iterationsschritt bleiben für jeden Würfel der Kantenlänge $3^{\mathrm{i}-1} 20$ kleinere Würfel der Kantenlänge $3^{-i}$ stehen. Die Dimension von $\log 20 / \log 3$ entspricht einem Massenfraktal. Für die Poren (in Abb. 2.4 weiß dargestellt) ist der Sachverhalt komplizierter. Das Volumen der Poren $\mathrm{V}_{\mathrm{P}}$ beträgt bei der $\mathrm{n}$-ten Iteration

$$
\mathrm{V}_{\mathrm{P}, \mathrm{n}}=7 \sum_{\mathrm{i}=1}^{\mathrm{n}}\left(20^{\mathrm{i}-1} \cdot 3^{-3 \mathrm{i}}\right)
$$


bei jedem Iterationsschritt kommt eine Porenklasse hinzu. Wird die Iteration beliebig oft durchgeführt, geht die Masse der festen Substanz gegen Null bzw. die der Poren gegen 1. Zwischen beiden Phasen befindet sich die Porenraumgrenzfläche.

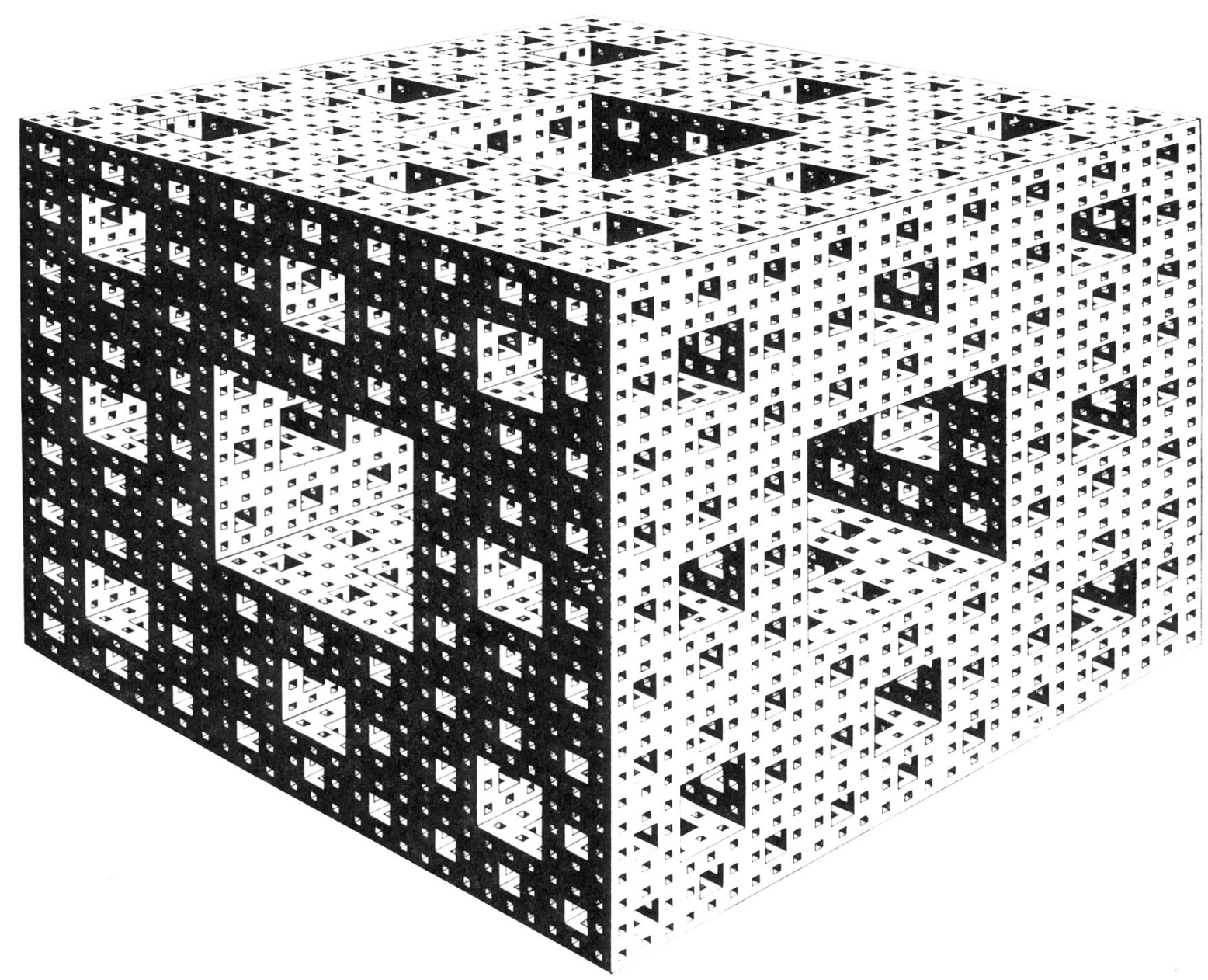

Abb. 2.4: Der Menger Schwamm besteht aus einem Würfel, der mit der ersten Iteration in 27 Würfel zerteilt wird, von denen 20 Masse besitzen und 7 leer sind. Aus Mandelbrot, 1983.

Die oben angeführten Beispiele stellen mathematisch erzeugte fraktale Muster dar. Hier kann die fraktale Dimension einfach nach obigen Gleichungen berechnet werden kann, wenn Skalierungsfaktor und Massenverhältnis bekannt sind.

Zur Messung eines natürlichen Fraktals, bei dem immer eine natürliche Grenze, wie z. B. durch die Größe von Molekülen verursacht, vorhanden ist, muß die Abweichung vom angelegten Maßstab berücksichtigt werden. Ein klassisches Beispiel ist die Messung der Länge der Küste von England (Richardson 1961, Mandelbrot 1967). Je größer der verwendete Maßstab (bzw. je kleiner der gedachte angelegte Zollstock), desto größer wird die ermittelte Länge. Wie oben ausführlich dargestellt, besteht für Fraktale ein direkter mathematischer Zusammenhang in Form eines Potenzgesetzes zwischen dem verwendeten Maßstab (bzw. der Länge des Zollstocks $\varepsilon$ ) und der ermittelten Länge. Für die Küste von England fand Richardson bereits 1939, publiziert 1961, die empirische Formel $\mathrm{L}(\varepsilon)=\mathrm{N}(\varepsilon) \varepsilon \propto \varepsilon^{-\mathrm{D}}$, was den Gleichungen 2.1 und 2.8 entspricht. Nach ihm wird die logarithmische Auftragung zur Ermittlung der Steigung Richardson-Plot genannt. Mit bildanalytischen Methoden kann die fraktale Dimension natürlicher Objekte gemessen 
werden. Dabei erhalten wir je nach verwendeter Methode unterschiedliche fraktale Dimensionen, z.B die Boxcounting-, Dilatation- und Yardstick-Dimension. Eine detaillierte Beschreibung der verwendeten Methoden findet sich in Kap. 4.5.

In der Praxis ist die fraktale Dimension von Bedeutung für die Charakterisierung komplexer Strukturen durch die Messung der Kennzahl D, die sich in ihrer Aussage von bisher verfügbaren Parametern unterscheidet (z. B. Kaye 1989). Darüber hinaus kann die fraktale Geometrie herangezogen werden, um die dynamischen Prozesse zu verstehen, die der Bildung natürlicher irregulärer Strukturen zugrunde liegen. Dieser Schritt von der gemessenen fraktalen Dimension zum kausalen Verständnis eines Prozesses (Strukturbildung und -veränderung) steckt noch in den Anfängen (Korvin 1992, Kruhl 1994). Ansätze existieren z. B. für Strukturbildungsprozesse natürlicher fraktaler biologischer Objekte (Weibel 1991).

\subsubsection{Fraktale in der Bodenkunde}

Im Bereich der Bodenkunde existieren unterschiedliche Ansätze für die Anwendung der fraktalen Geometrie, die in Korvin (1992), Anderson et al. (1998), Baveye \& Boast (1998), und Crawford et al. (1999) im Überblick dargestellt sind. Auch hier sind beide Herangehensweisen (mathematische Modellierung und direkte Messung) vertreten. Mathematische Modellierungsansätze beziehen sich meist auf die Parametrisierung von Retentions- und Leitfähigkeitsfunktionen mit einer fraktalen Dimension D im Exponenten. Dabei werden bereits Modellvorstellungen über die Bodenstruktur zugrunde gelegt. Normalerweise wird die Retentionsfunktion (s. Kap. 2.2) an im Labor oder auch im Gelände aufgenommene Daten des Wassergehalts in Abhängigkeit von der Wasserspannung angepaßt. Zum Einsatz kommen empirische Funktionen wie z. B. bei Brooks \& Corey (1964) und van Genuchten (1980). Die ungesättigte Wasserleitfähigkeit kann aus der Retentionskurve berechnet werden. Dafür werden physikalisch basierte Modellvorstellungen der Porenstruktur verwendet (Mualem 1976, van Genuchten 1980). Die Bodenstruktur selbst wird seltener untersucht. Auch Korn- und Aggregatgrößenverteilungen können als fraktal skalierende Summenkurven dargestellt werden. Die direkte bildanalytische Messung fraktaler Eigenschaften der Bodenstruktur, wie sie in dieser Arbeit vorgestellt wird, wurde erst von wenigen Autoren untersucht

Eine sehr klare Differenzierung über die verschiedenen fraktalen Eigenschaften, die von diversen Autoren untersucht wurden, bieten Perrier et al. (1999) in ihrer Arbeit über die Generalisierung verschiedener Modellansätze (Massenfraktal, Porenfraktal und Fraktal der Porenraumgrenzfläche). Es kann in theoretische und praktische Modellierungsansätze mittels

(i) fraktaler Massenverteilung (Rieu \& Sposito 1991c, Crawford et al. 1993b, Crawford 1994, Crawford et al. 1995, Bird et al. 1996),

(ii) fraktaler Porenverteilung (Ahl \& Niemeyer 1989, Rieu \& Sposito 1991a,b,c, Perrier et al. 1995, Bird et al. 1996, Perfect et al. 1996, Bird \& Dexter 1997, Giménez et al. 1997, Niemeyer \& Machulla 1999),

(iii) fraktaler Grenzflächen (Bird et al. 1996, Giménez et al. 1997; Bartoli et al. 1999),

(iv) fraktaler Partikelgrößenverteilung (number/size distribution; Tyler \& Wheatcraft 1989, Bartoli et al. 1991, Crawford et al. 1993b, Perrier et al. 1995, Comegna et al. 1998), 
(v) fraktaler Aggregatverteilung (Rieu \& Sposito 1991a,b, Crawford et al. 1997) unterschieden werden.

Perrier et al. (1999) entwickelten, basierend auf einem Ansatz von Neimark (1989), ein Poren-Matrix Modell, welches die Vorteile einerseits einer definierten Porosität und andererseits eine mathematisch exakte Beschreibung der Zusammenhänge zwischen den oben aufgeführten Verteilungen bietet. Bird \& Dexter (1997) benutzen ein fraktales Modell um ein theoretisches Porensystem zu beschreiben. Die Anordnung der Poren erfolgt dabei zufällig (random network). Die Autoren zeigen, dass aus der Retentionskurve nicht ohne Vorbehalt auf die Porengrößenverteilung geschlossen werden kann und umgekehrt die Porengrößenverteilung nicht ausreicht, um den Wassertransport zu beschreiben. Der Grund liegt in der Konnektivität der Poren, Porenbäuche können erst entwässern, wenn die Wasserspannung der sie verbindenden Porenhälse erreicht ist. Moreau et al. (1999) kommen durch bildanalytische Messungen im zwei- und dreidimensionalen Raum zu einem ähnlichen Ergebnis. Während die fraktale Dimension eines präparierten Bodenblockes mit wenigen Schnitten erfaßt werden kann, trifft dies für die Konnektivität nicht zu.

Die direkte Messung fraktaler Dimensionen durch digitale Bildanalyse kann unterschieden werden in die Messung der

(i) fraktalen Massenverteilung (Bartoli et al. 1991, Crawford et al. 1993b),

(ii) fraktalen Porenverteilung (Bartoli et al. 1991, Crawford et al. 1993a, Anderson et al. 1996, Giménez et al. 1997),

(iii) fraktalen Partikelgrößenverteilung (number/size distribution),

(iv) fraktalen Porengrößenverteilung (number/size distribution),

(v) fraktalen Dimension der Porenraumgrenzfläche (Pachepsky et al. 1996, Giménez et al. 1997, Bartoli et al. 1999, Dathe et al. 2001)

und in die Messung der

(vi) fraktalen Dimension der Aggregatgrößenverteilung (Crawford et al 1993b).

Ogawa et al. (1999) messen die fraktale Dimension der Grenzfläche eines mit einem Farbtracer erzeugten Musters von bevorzugten Fließbahnen. Crawford et al. (1993a) messen das fraktale Muster von Pilzhyphen im Boden, das dem der DLA (Diffusionlimited aggregation, Witten \& Sander 1981) nahekommt. Bei den verschiedenen zu messenden Größen werden unterschiedliche Messverfahren angewendet, von denen eine Auswahl in Baveye \& Boast (1998) mathematisch hergeleitet wird. Im Grunde genommen wird mit jeder Meßmethode eine spezifische fraktale Dimension ermittelt. Verschiedene Autoren messen mit unterschiedlichen Methoden wie

(i) Boxcounting (Bartoli et al. 1991, Bartoli et al. 1999, Moreau et al. 1999, Ogawa et al. 1999),

(ii) Dilatation (Dathe et al. 2001),

(iii) der Bestimmung der spektralen Dimension (Beschreibung der virtuellen Bewegung eines Teilchens durch ein Netzwerk; Crawford et al 1993a, Anderson et al. 1996, Giménez et al. 1997), 
(iv) Messung des Verhältnisses Fläche/Umfang von einzelnen Partikeln (Orford und Whalley 1983), oder um die Oberflächenrauhigkeit von Poren zu bestimmen (Pachepsky et al. 1996, Crawford et al 1997, Giménez et al. 1997)

(v) Messung des Verhältnisses Masse/Radius (Crawford et al. 1993a, Crawford et al. 1997),

(vi) Porensehnenverteilung (pore chord distribution; Bartoli et al. 1999),

(vi) Openingverfahren (Erosion gefolgt von Dilatation; Moreau et al. 1999),

(vii) Closingverfahren (Dilatation gefolgt von Erosion; Anderson et al. 1996)

(viii) Bestimmung der Informationsdimension (Ogawa et al. 1999) und der

(ix) Bestimmung der Korrelationsdimension (Ogawa et al. 1999).

Auf die in dieser Arbeit angewendeten Methoden Boxcounting, Dilatation und Opening wird in den Kapiteln 4.4 und 4.6 genauer eingegangen. Die Übersicht aller Methoden soll einen Eindruck davon vermitteln, wie unterschiedlich die Herangehensweisen einzelner Autoren sind und wie schwierig es sein kann, Ergebnisse zu vergleichen die mit verschiedenen Methoden für unterschiedliche Meßobjekte erzielt wurden. In dieser Hinsicht bieten Anderson et al. (1998) einen bemerkenswerten Überblick.

Bartoli und Mitarbeiter (1991, 1999) verwenden zusätzlich die Quecksilberporosimetrie. Celis et al. (1996) untersuchen die Oberflächen von Tonen (Kaolinit und Montmorillonit) mit Quecksilberporosimetrie und Stickstoffadsorption. Bei einem höheren Anteil an zugefügten Eisenhydroxiden messen sie eine höhere fraktale Dimension der inneren Oberfläche. Pape et al. (1987) stellen das ,pigeon-hole model“ vor, das als fraktales Netzwerk beschrieben werden kann und durch eine fraktale Oberfläche charakterisiert wird. Es bezieht sich allerdings auf geologische Formationen (Sandstein) und nicht auf den Boden. Später geht Pape (1996) auf zeitliche Prozesse ein, die sich in räumlichen Strukturen widerspiegeln und ebenfalls durch fraktale Geometrien beschreibbar sind.

\subsection{Bodenphysikalische Grundlagen}

Theoretische Aspekte der Bodenphysik sollen hier nur in Kürze beschrieben werden, da der Schwerpunkt der Arbeit auf der digitalen Bildanalyse liegt. Die Bodenphysik betrachtet die physikalischen Gesichtspunkte von Böden. Der Boden stellt ein poröses Medium dar, das aus Mineralkörnern und mit Wasser oder Bodenluft gefüllten Poren besteht. Organische Substanz, Mikro- und Makroorganismen tragen wesentlich zur Strukturierung bei, sollen hier jedoch nicht weiter betrachtet werden.

Tab. 2.1: Korngrößenfraktionen nach der deutschen Nomenklatur. Verändert aus Schachtschabel et al. 1989.

\begin{tabular}{|c|c|c|c|}
\hline Fraktion & fein & mittel & grob \\
\hline \multicolumn{4}{|c|}{ Korngrößendurchmesser } \\
\hline Ton & $<0,2 \mu \mathrm{m}$ & $<0,63 \mu \mathrm{m}$ & $<2,0 \mu \mathrm{m}$ \\
\hline Schluff & $<6,3 \mu \mathrm{m}$ & $<20 \mu \mathrm{m}$ & $<63 \mu \mathrm{m}$ \\
\hline Sand & $<200 \mu \mathrm{m}$ & $<630 \mu \mathrm{m}$ & $<2000 \mu \mathrm{m}$ \\
\hline
\end{tabular}


Die mineralischen Bestandteile werden in einer logarithmischen Skala ihren Korngrößendurchmessern entsprechend in die Bodenarten Ton, Schluff und Sand unterteilt (s. Tab. 2.1).

Das Porensystem im Boden ist ein komplexes dreidimensionales Netzwerk, wobei die Poren primär durch die Anordnung der mineralischen Partikel entstehen. So kann durch Bestimmung der Korngrößenverteilung die Verteilung einzelner Porengrößenklassen abgeschätzt werden (Hartge \& Horn 1999). Die direkte Messung des Porensystems entzieht sich jedoch dem Betrachter. Eine relativ einfach zu erhebende Kenngröße ist die Dichte, wobei in Lagerungsdichte $\mathrm{d}_{\mathrm{B}}$ und Dichte der Festsubstanz $\mathrm{d}_{\mathrm{F}}$ unterschieden wird. Sind beide bekannt, so kann nach

$$
\mathrm{V}_{\mathrm{P}}=1-\frac{\mathrm{d}_{\mathrm{B}}}{\mathrm{d}_{\mathrm{F}}}
$$

das Porenvolumen $V_{P}$ berechnet werden. Das Porensystem ist für Transportprozesse im Boden, wie Gas- und Wassertransport und den damit verbundenen Stofftransport von Bedeutung. Von Relevanz sind diese Transportprozesse für die Grundwasserneubildung, den Schadstofftransport und für die Versorgung von Pflanzen mit Wasser und Nährstoffen. Die Grenzen zwischen den Porengrößenbereichen sind an charakteristische Kennwerte des Wasserhaushaltes angelehnt (Schachtschabel et al. 1989 und Tab. 2.2 sowie Abb. 2.5). Das Wasser in Poren $>50 \mu \mathrm{m}$ Durchmesser unterliegt vor allem der Gravitation, daher sind diese Poren meist wasserfrei. Die Porendurchmesser von 50 und $10 \mu \mathrm{m}$ entsprechen der oberen und unteren Entwässerungsgrenze bei Feldkapazität ${ }^{1}$ (pF 1,8-2,5). Die Entwässerungsgrenze des permanenten Welkepunkts ( $\mathrm{pF} 4,2)$ entspricht einem Porendurchmesser von $0,2 \mu \mathrm{m}$, d. h., Wasser in Poren $<0,2 \mu \mathrm{m}$ ist in der Regel nicht pflanzenverfügbar, da die Feinwurzeln einen Durchmesser $>0,2 \mu \mathrm{m}$ aufweisen.

Tab. 2.2: Einteilung der Porengrößenbereiche nach dem Porendurchmesser und der korrelierenden Wasserspannung. Verändert aus Schachtschabel et al. 1989.

\begin{tabular}{|c|c|c|c|}
\hline Porengrößenbereich & $\begin{array}{c}\text { Durchmesser } \\
{[\mu \mathrm{m}]}\end{array}$ & $\begin{array}{c}\text { Wasserspannung } \\
{[\mathrm{hPa}]}\end{array}$ & $\mathrm{pF}-$ Wert \\
\hline weite Grobporen & $>50$ & $<60$ & $0-1,8$ \\
\hline enge Grobporen & $50-10$ & $60-300$ & $1,8-2,5$ \\
\hline Mittelporen & $10-0,2$ & $300-15000$ & $2,5-4,2$ \\
\hline Feinporen & $<0,2$ & $>15000$ & $>4,2$ \\
\hline
\end{tabular}

Streng genommen müßten die Durchmesser der in Tab. 2.2 definierten Poren mit Äquivalentdurchmesser bezeichnet werden. Ihr Durchmesser wird nach der Kapillarengleichung berechnet, die einen umgekehrt proportionalen Zusammenhang zwischen der Steighöhe einer Flüssigkeit in Abhängigkeit vom Radius der Kapillare beschreibt und für kreiszylindrische Poren gilt:

\footnotetext{
${ }^{1}$ Die Feldkapazität ist definiert als der Wassergehalt, bei dem sich im Boden nach länger andauernden Niederschlägen ein gleichmäßiges hydraulisches Potential $\left(\Psi_{\mathrm{H}}=0\right)$ einstellt (Schachtschabel et al. 1989).
} 


$$
\begin{array}{lll}
\mathrm{h}_{\mathrm{c}}= & \frac{2 \sigma \cos \beta}{\rho g \mathrm{r}_{\mathrm{c}}} \\
& \\
\text { mit } & \mathrm{h}_{\mathrm{c}}: & \text { Steighöhe der Wassersäule in der Kapillare }[\mathrm{m}] \\
& \sigma: & \text { Grenzflächenspannung }[\mathrm{N} / \mathrm{m}] \\
& \beta: & \text { Benetzungswinkel }\left[{ }^{\circ}\right] \\
\rho: & \text { Dichte der Flüssigkeit }\left[\mathrm{kg} / \mathrm{m}^{3}\right] \\
\mathrm{g}: & \text { Erdbeschleunigung }\left[\mathrm{m} / \mathrm{s}^{2}\right] \\
\mathrm{r}_{\mathrm{c}}: & \text { Kapillarenradius }[\mathrm{m}]
\end{array}
$$

Bei einer mittleren Grenzflächenspannung von Wasser zu Luft von $\sigma=72,7 \cdot 10^{-3} \mathrm{~N} / \mathrm{m}$, vollständiger Benetzung $(\cos \beta=1)$ und der Erdbeschleunigung von $9,81 \mathrm{~m} / \mathrm{s}^{2}$ gilt für den Kapillarenradius $r_{c}[\mathrm{~m}]$ bzw. den Äquivalentporendurchmesser $D_{c}[\mu \mathrm{m}]$ näherungsweise:

$$
\mathrm{r}_{c} \approx \frac{15 \cdot 10^{-6}}{\mathrm{~h}_{\mathrm{c}}} \quad \text { bzw. } \quad \mathrm{D}_{\mathrm{c}} \approx \frac{30}{\mathrm{~h}_{\mathrm{c}}}
$$

Das Wasser im Boden unterliegt unterschiedlichen Kräften wie der Gravitationskraft und Kohäsions- und Adhäsionskräften, die im Potentialkonzept beschrieben werden (Buckingham 1907). Das Potential ist hierbei definiert als die Arbeit, die notwendig ist um eine Einheitsmenge Wasser von einem gegebenen Punkt eines Kraftfeldes zu einem Bezugspunkt zu transportieren (Schachtschabel et al. 1989). Roth (1996) beschreibt das Bodenwasserpotential als Dichte der potentiellen Energie des Bodenwassers. Das Gesamtpotential $\psi$ wird als Summe der Teilpotentiale definiert:

$$
\psi=\psi_{\mathrm{m}}+\psi_{\mathrm{z}}+\psi_{\mathrm{g}}+\psi_{\mathrm{o}}
$$

Das Gaspotential $\psi_{\mathrm{g}}$ muß nicht berücksichtigt werden wenn der Luftdruck im Boden mit dem des Bezugsniveaus übereinstimmt. Das osmotische Potential $\psi_{0}$ ist für Böden mit salzhaltiger Bodenlösung und bei der Bestimmung von Transportprozessen relevant. So werden in der Praxis häufig die beiden leicht zu bestimmenden Größen Matrixpotential $\left(\psi_{\mathrm{m}}\right)$ und Gravitationspotential $\left(\psi_{\mathrm{z}}\right)$ zum hydraulischen Potential $\psi_{\mathrm{H}}$ zusammengefaßt:

$$
\psi_{\mathrm{H}}=\psi_{\mathrm{m}}+\psi_{\mathrm{z}}
$$

Das Matrixpotential ist die Energie die benötigt wird, um Wasser in die poröse Matrix zu bringen. Diese Energie kann mit der Druckdifferenz zwischen Wasser- und Luftphase gleichgesetzt werden und beeinflusst die Krümmung der Grenzfläche bzw. die Steighöhe des Wassers in einer Kapillaren, was auf Gl. 2.20. zurückverweist (Roth 1996). Das Matrixpotential trägt die physikalische Einheit eines Druckes [hPa]. Mit einem positiven Vorzeichen versehen wird auch der Begriff Wasserspannung verwendet. $1 \mathrm{~cm}$ WS (Wassersäule) $\approx 0,981 \mathrm{hPa}$. Im Gravitationspotential wird der Abstand zum Grundwasserspiegel ausgedrückt. Das hydraulische Potential geht in die Wassertransportgleichung ein, welche eine spezielle Form der Fokker-Planck-Gleichung darstellt. 


$$
\frac{\partial \theta}{\partial t}=\frac{\partial}{\partial x}\left(K \frac{\partial \psi_{H}}{\partial x}\right)+\frac{\partial}{\partial y}\left(K \frac{\partial \psi_{H}}{\partial y}\right)+\frac{\partial}{\partial z}\left(K \frac{\partial \psi_{H}}{\partial z}\right)
$$

Um diese Gleichung lösen zu können, müssen Werte für die ungesättigte hydraulische Leitfähigkeit K, den Wassergehalt $\theta$ und das hydraulische Potential $\psi_{\mathrm{H}}$ vorliegen. Diese Werte liefert die Funktion $\theta\left(\psi_{\mathrm{m}}\right)$, die auch als Retentionskurve, Wasserspannungskurve oder pF-Charakteristik (s. Abb. 2.5) bezeichnet wird und für jeden Bodenhorizont charakteristisch ist. Retentionskurven können im Labor durch die gravimetrische Messung des Wassergehaltes in Abhängigkeit von einer angelegten Druckdifferenz oder im Gelände durch die gleichzeitige Messung von Wassergehalt (z. B. durch TDR-Sonden, s. Stacheder 1996) und Matrixpotential bestimmt werden. Um eine kontinuierliche Funktion zu erhalten werden empirische Funktionen an erhobene Meßwerte angepaßt (Brooks \& Corey 1964, van Genuchten 1980, s. Kap. 2.2.1) oder aus Pedotransferfunktionen entwickelt (Tietje \& Tapkenhinrichs 1993). Die ungesättigte Leitfähigkeit kann im Labor bestimmt werden, was jedoch sehr zeitaufwändig und auf den Messbereich von Tensiometern (bis ca. $-800 \mathrm{hPa}$ ) beschränkt ist (s. Abschnitt 4.7.4). Daher existieren bereits seit Mitte des 20. Jahrhunderts Ansätze, die stark vom Wassergehalt abhängige ungesättigte Wasserleitfähigkeit aus der Beziehung zwischen Wassergehalt- und Wasserspannung bzw. Matrixpotential zu berechnen. Dies führte letztlich auch zur Entwicklung der Retentionsfunktionen (s. Kap. 2.2.1).

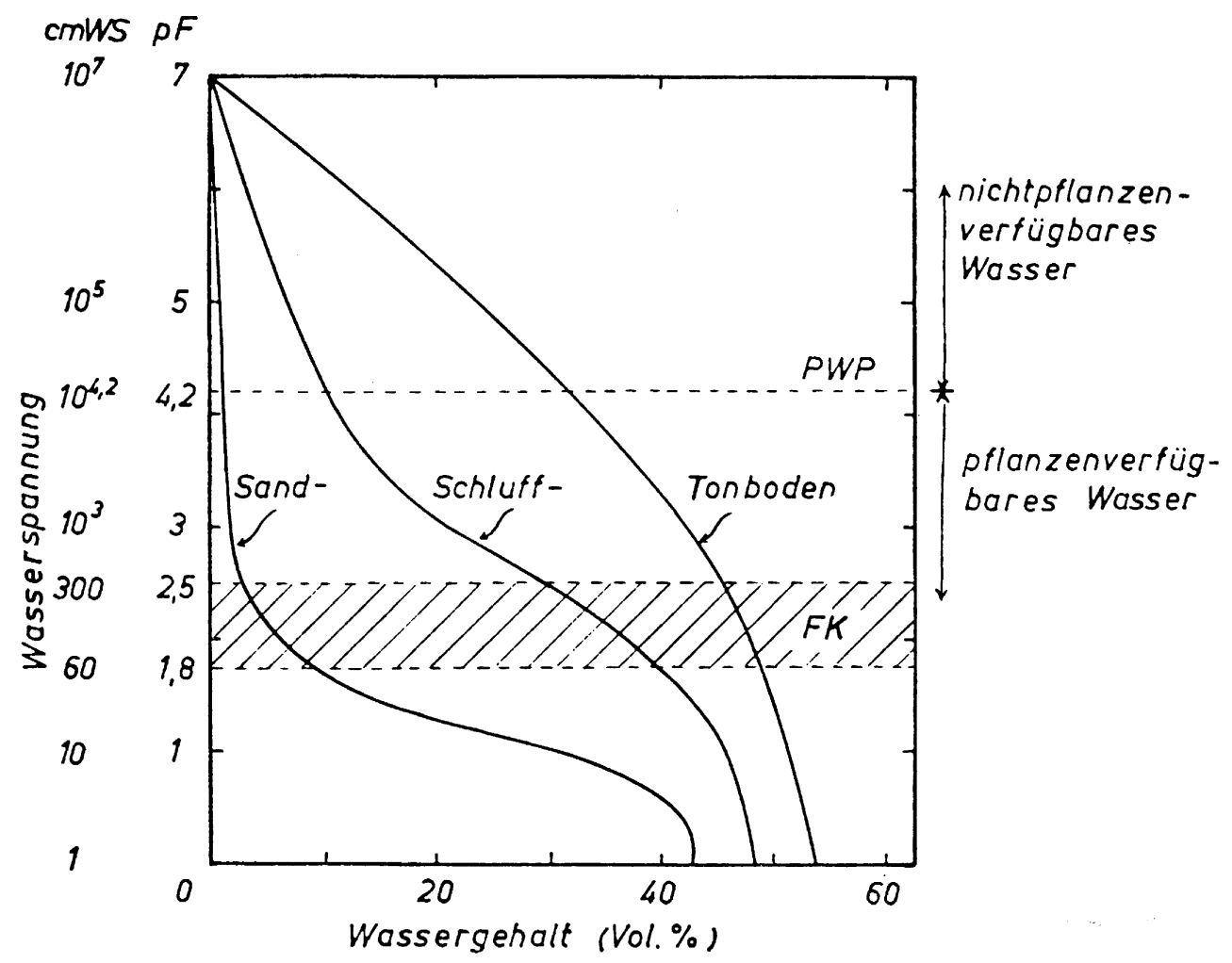

Abb. 2.5: Schematische Retentionskurven eines Sand-, Schluff- und Tonbodens und Markierung des permanenten Welkepunktes PWP und der Feldkapazität FK. Aus Schachtschabel et al. 1989, S. 178.

Bei der Erstellung von Retentionskurven im Labor sind in jüngster Zeit zunehmend dynamische Effekte Gegenstand der Untersuchungen (Dünger et al. 1995, Schultze \& Durner 1997). Je nach Größe der verwendeten Stechzylinder und Geschwindigkeit der 
Entwässerung zeigen die Kurven einen unterschiedlichen Verlauf. Bei kleinräumigen Messungen im Gelände (Göttlein et al. 1996) sowie an Einzelaggregaten (Junkersfield \& Horn 1997) werden ähnliche Effekte beobachtet. Gerade die übliche Methode, aus der Retentionskurve Rückschlüsse auf die Porengrößenverteilung $\mathrm{zu}$ ziehen, ist mit methodischen Fehlern behaftet (Ehlers et al. 1995, Bird \& Dexter 1997, Vogel 2000). So wird zunehmend versucht, Retention und Leitfähigkeit direkt aus der physikalischen Bodenstruktur abzuleiten. Vogel (1996), Vogel \& Kretzschmar (1996) sowie Vogel \& Roth (1997) untersuchen den Porenraum quasi dreidimensional und entwickeln aus der bildanalytisch nach dem Opening-Verfahren erfaßten Struktur und Kontinuität ein Netzwerkmodell, mit dem Wassertransport im Boden simuliert werden kann. Weitere Möglichkeiten zur Erfassung des 3D Porenraums wie die Untersuchung der Porenstruktur mittels Computertomographie (Heijs \& Lange 1997) erreichen nicht die in unserem Ansatz erforderliche hohe Auflösung der Abbildungsverfahren. Die hier vorgestellte Arbeit zielt darauf $a b$, durch die direkte Messung der fraktalen Dimension der Porenstruktur eine Kennzahl zu erheben, um

(i) die Komplexität des Systems durch eine charakteristische Kennzahl D zu beschreiben,

(ii) unterschiedliche Böden und ihre Horizonte durch diese Maßzahl quantitativ beschreiben zu können und

(iii) einen Parametersatz zu liefern, der zur Beschreibung von Retention und Leitfähigkeit in Simulationsmodelle eingehen könnte.

\subsubsection{Parametrisierung von Retentions- und Leitfähigkeitskurven}

Um den Wassertransport im Boden berechnen zu können (s. Gl. 2.18), muss die Funktion der ungesättigten hydraulischen Leitfähigkeit in Abhängigkeit vom Wassergehalt oder von der Wasserspannung vorliegen. Das Modell von Burdine (1953) geht davon aus, dass nur die Poren am Transport teilnehmen, die der Kapillarengleichung entsprechend (G1. 2.20) bei einem bestimmten Unterdruck $\psi$ noch mit Wasser gefüllt sind. Es beschreibt die relative ungesättigte Wasserleitfähigkeit $\mathrm{K}_{\mathrm{r}}$ als Funktion des effektiven Wassergehaltes $\theta$ für gemessene Wassergehalts/Wasserspannungsbeziehungen.

$$
\mathrm{K}_{\mathrm{r}}(\theta)=\Theta^{2} \int_{0}^{\theta} \frac{\mathrm{d} \theta}{\psi^{2}} / \int_{0}^{\theta_{\mathrm{s}}} \frac{\mathrm{d} \theta}{\psi^{2}}
$$

$$
\theta=\theta-\theta_{\mathrm{r}}
$$

Die relative Wassersättigung $\Theta$ berechnet sich nach

$$
\Theta=\frac{\theta-\theta_{\mathrm{r}}}{\theta_{\mathrm{s}}-\theta_{\mathrm{r}}}
$$

aus dem aktuellen Wassergehalt $\theta$, dem Wassergehalt bei Sättigung $\theta_{\mathrm{s}}$ und dem residualen Wassergehalt $\theta_{\mathrm{r}}$. Der residuale Wassergehalt ist definiert als der Wassergehalt, bei dem die Leitfähigkeit und $\mathrm{d} \theta / \mathrm{d} \psi$, also die Steigung der Retentionskurve bzw. die spezifische Wasserkapazität, für hohe Saugspannungen gegen Null gehen. 
Mualem (1976) entwickelte ein neues Modell für die Vorhersage der Wasserleitfähigkeit aus Retentionsdaten. Es beschreibt die relative ungesättigte Wasserleitfähigkeit als quadrierten Quotienten zweier Integrale, der mit einem Korrekturfaktor multipliziert wird:

$$
\mathrm{K}_{\mathrm{r}}(\theta)=\Theta^{\mathrm{n}}\left[\int_{0}^{\theta} \frac{\mathrm{d} \theta}{\psi} / \int_{0}^{\theta_{\mathrm{S}}} \frac{\mathrm{d} \theta}{\psi}\right]^{2}
$$

Der Quotient drückt das Verhältnis zwischen wassergefüllten Poren des zum aktuellen Matrixpotentials $\psi$ gehörenden Radius zum gesamten Porenvolumen aus, wenn die Porenverteilung an der Ober- und Unterseite einer Bodenschicht betrachtet wird. Der Korrekturterm steht für die Tortuosität der Fließwege und stellt eine Funktion des Wassergehaltes dar. Brooks und Corey (1964) entwickelten eine Funktion, die die relative Wassersättigung $\Theta$ als Quotienten des Matrixpotentials beim Lufteintrittspunkt $\psi_{\mathrm{b}}$ (bubble pressure, air entry value) und des aktuellen Matrixpotentials mit einem Exponenten $\lambda$, der einen Porengrößenindex darstellt, beschreibt:

$$
\Theta=\left\{\begin{array}{cl}
\left(\frac{\psi_{b}}{\psi}\right)^{\lambda} & \text { für } \psi \leq \psi_{b} \\
1 & \text { für } \psi>\psi_{b}
\end{array}\right.
$$

Nach dieser Modellvorstellung bleibt der Boden bis zum Erreichen des Lufteintrittspunktes wassergesättigt. Die Funktion hat den Nachteil, dass sie nicht stetig ist, was ihren Einsatz in Simulationsmodellen erschwert. Der Wert für $\lambda$ ist groß für Böden, die eine gleichförmige Porengrößenverteilung und klein für Böden, die eine weite Spanne der Porengrößenverteilung aufweisen.

Eine stetige Funktion zur Beschreibung des Wassergehaltes in Abhängigkeit des Matrixpotentials stellte van Genuchten (1980) vor:

$$
\Theta=\left\{\begin{array}{cl}
\frac{1}{\left(1+|\alpha \psi|^{\mathrm{n}}\right)^{\mathrm{m}}} & \text { für } \psi \leq 0 \\
1 & \text { für } \psi>0
\end{array}\right.
$$

Darin sind $\alpha, \mathrm{n}$ und $\mathrm{m}$ Anpassungsparameter, die nicht direkt physikalisch messbar sind. Es kommt ihnen jedoch folgende Bedeutung $\mathrm{zu}$ : $\alpha$ ist invers korreliert zum Lufteintrittspunkt und n drückt den Verlauf der Retentionskurve im Bereich der größten Steigung aus und ist damit eine Maßzahl für die Porengrößenverteilung. Der Parameter $m$ wird üblicherweise mit $\mathrm{m}=1+1 / \mathrm{n}$ angepasst. Zwischen den Parametern der van Genuchten Funktion und der Anpassung nach Brooks und Corey besteht somit folgende Beziehung:

$$
\begin{aligned}
& \alpha=\psi_{\mathrm{b}}^{-1} \\
& \mathrm{n}=1+\lambda .
\end{aligned}
$$

Die ungesättigte Wasserleitfähigkeit kann durch Kombination der G1. 2.27 und 2.29 berechnet werden (van Genuchten 1980) 


$$
\mathrm{K}_{\mathrm{r}}(\Theta)=\Theta^{\frac{1}{2}}\left[1-\left(1-\Theta^{1 / \mathrm{m}}\right)^{\mathrm{m}}\right]^{2}
$$

oder, als Funktion des Matrixpotentials, indem Gl. 2.29 in 2.30 eingesetzt wird:

$$
\mathrm{K}_{\mathrm{r}}(\psi)=\frac{\left\{1-(\alpha|\psi|)^{\mathrm{n}-1} \cdot\left[1+(\alpha|\psi|)^{\mathrm{n}}\right]^{-\mathrm{m}}\right\}^{2}}{\left[1+(\alpha|\psi|)^{\mathrm{n}}\right]^{\mathrm{m} / 2}}
$$

Die aktuelle Wasserleitfähigkeit ergibt sich dann aus dem Produkt aus relativer und gesättigter Leitfähigkeit:

$$
\mathrm{K}=\mathrm{K}_{\mathrm{r}} \cdot \mathrm{K}_{\mathrm{s}}
$$

Die Gleichungen nach Mualem und van Genuchten stellen die in der Bodenphysik und Hydrologie wohl am häufigsten verwendete Methode zur Parametrisierung von Retention und Leitfähigkeit dar. 



\section{$3 \quad$ Material und Präparation}

\subsection{Herkunft des verwendeten Bodenmaterials}

Das Bodenmaterial stammt aus der Schichtstufenlandschaft zwischen Leinegraben und unterem Eichsfeld südöstlich von Göttingen. Die Proben wurden in unmittelbarer Nähe eines mehrere Meter mächtigen Aufschlusses gewonnen, der sich an der SüdostAbdachung des Stangenberges zwischen Reinhausen und Bremke an der B 568 befindet (s. Abb. 3.1). Es handelt sich um eine offengelassene ehemalige Lehmgrube. Das Profil befindet sich auf einer Höhe von ca. 255 m über NN. Der Bodentyp ist eine Parabraunerde auf Löß, welche durch Verwitterung und Tonverlagerungsprozesse aus im Jungwürm abgelagertem Material entstanden ist. Der Löß liegt hier auf dem mittleren Buntsandstein (sm1) auf. In der Baumschicht herrschen ca. 40 Jahre alte Fichten vor, vereinzelt finden sich auch Buchen. Eine deckende Kraut- oder Strauchschicht ist nicht vorhanden. Das Profil zeichnet sich besonders durch seine Mächtigkeit aus und ist bis in eine Tiefe von $260 \mathrm{~cm}$ entkalkt. In $160 \mathrm{~cm}$ Tiefe befindet sich ein schmales dunkles Bändchen. Darunter, bis zur Entkalkungstiefe, befinden sich parallele Bänder unterschiedlicher Färbung, wobei die dunkleren Bänder einen rötlicheren Farbton aufweisen als der umgebende Löß. Die Proben für die bildanalytischen Untersuchungen wurden in 70-80 $\mathrm{cm}$ Tiefe aus dem Tonanreicherungshorizont entnommen (s. Tab. 3.1 und 3.2).

Tab. 3.1: Feldanspracheergebnisse und bodenchemische Merkmale der untersuchten Parabraunerde. Die $\mathrm{pH}$ Werte stammen aus Glasow (2000), Kohlenstoff (C) und Stickstoff (N) wurden mit einem CHN1000 (LECO) bestimmt, und beziehen sich auf lufttrockenen Boden. Die Farbe wurde nach der Munsell Farbtafel und das Gefüge entsprechend der Bodenkundlichen Kartieranleitung (AG Boden 1994) bestimmt. Verändert aus Friedrichs (1999).

\begin{tabular}{|c|c|c|c|c|c|c|}
\hline \multirow{2}{*}{$\begin{array}{l}\text { Hori- } \\
\text { zont }\end{array}$} & \multirow{2}{*}{$\begin{array}{l}\text { Tiefe } \\
{[\mathrm{cm}]}\end{array}$} & \multirow[t]{2}{*}{$\mathrm{pH}$} & \multicolumn{2}{|c|}{ Gesamtgehalte $[\mathrm{g} / \mathrm{g} * 100]$} & \multirow[t]{2}{*}{ Farbe } & \multirow[t]{2}{*}{ Gefüge } \\
\hline & & & $\mathrm{C}$ & $\mathrm{N}$ & & \\
\hline $\mathrm{L}$ & \multirow{3}{*}{$5-0$} & - & - & - & - & - \\
\hline $\mathrm{O}_{\mathrm{F}}$ & & - & - & - & - & - \\
\hline $\mathrm{O}_{\mathrm{H}}$ & & - & - & - & - & - \\
\hline $\mathrm{A}_{\mathrm{h}}$ & $0-2$ & - & 15,33 & 0,31 & $7,5 \mathrm{YR} 2 / 2$ & - \\
\hline $\mathrm{A}_{\mathrm{e}}$ & $2-5$ & - & 8,01 & 0,17 & 7,5YR 6/1 & - \\
\hline $\mathrm{A}_{1}$ & $5-40$ & 4,23 & 0,51 & 0,13 & $7,5 \mathrm{YR} 4 / 3$ & subpolyedrisch, halboffen \\
\hline $\mathrm{B}_{\mathrm{t}}$ & $40-140$ & 4,88 & 0,21 & 0,03 & 7,5YR 4/6 & $\begin{array}{c}\text { polyedrisch-prisma-tisch, } \\
\text { geschlossen, } \\
\text { Nadelstichporen }\end{array}$ \\
\hline$B_{v t}$ & $140-160$ & 5,29 & 0,15 & 0,02 & 7,5YR 5/6 & $\begin{array}{l}\text { polyedrisch-prisma-tisch, } \\
\text { geschlossen, Bänder, } \\
\text { Nadelstichp, }\end{array}$ \\
\hline $\mathrm{B}_{\mathrm{v}} \mathrm{C}$ & $160-260$ & 5,60 & 0,12 & 0,02 & 7,5YR 6/6 & kohärent, Bänder \\
\hline $\mathrm{C}$ & $>260$ & 8,04 & 1,31 & 0,03 & 7,5YR 6/6 & $\begin{array}{c}\text { kohärent, Bänder, } \\
\text { karbonatisch }\end{array}$ \\
\hline
\end{tabular}




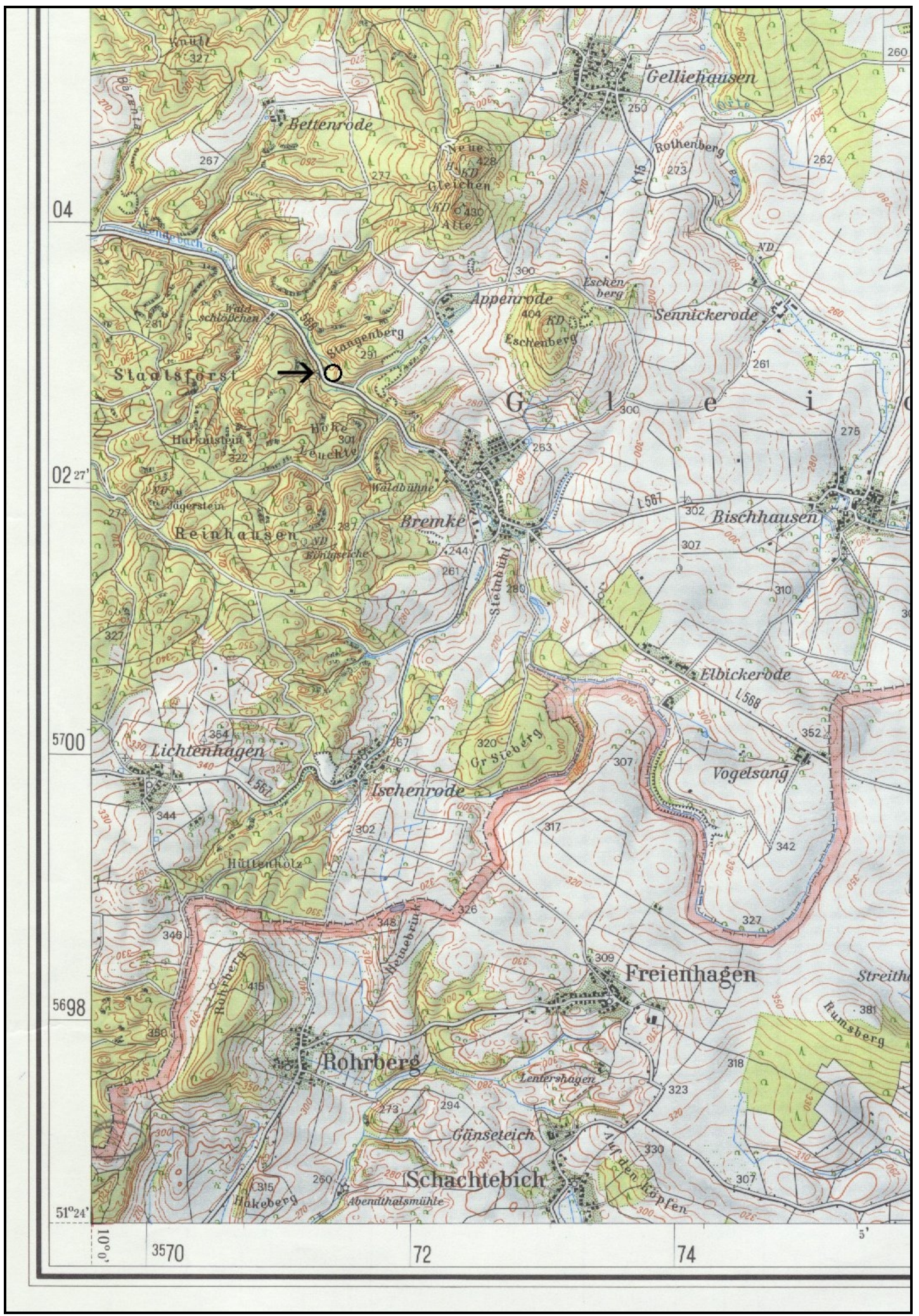

Abb. 3.1: Herkunft des verwendeten Bodenmaterials sudöstlich von Göttingen $(3571,46 ; 5702,85$ im GaussKrüger Gitter). Es handelt sich um ein stark reliefiertes Gelände aus der Schichtstufenlandschaft zwischen Leinetalgraben im Westen und unterem Eichsfeld im Osten. In den tieferen Lagen des Staatsforstes Reinhausen sowie im nördlich des Wendebachs gelegenen Privatwald tritt der mittlere Buntsandstein $\left(\mathrm{sm}_{1}\right)$ zutage. In einigen Senken finden sich teils sehr mächtige Lößablagerungen, wie an dem für diese Untersuchung gewählten Standort. Aus: Topographische Karte 1:50000, Blatt L4526, Duderstadt, Ausgabe 1987. 
Tab. 3.2: Im Labor ermittelte bodenphysikalische Parameter der Parabraunerde (Profil Waldschlößchen). Die Lagerungsdichte $\mathrm{L}_{d}$ wurde durch Wägen der Stechzylinder und die Textur nach der Pipettiermethode bestimmt. Die Wassergehalte entstammen der pF-Kurve. Die bodenphysikalischen Methoden werden in Kapitel 4.5 ausführlich beschrieben. Verändert aus Friedrichs (1999).

\begin{tabular}{|c|c|c|c|c|c|c|c|c|c|}
\hline \multirow{2}{*}{$\begin{array}{l}\text { Hori- } \\
\text { zont }\end{array}$} & \multirow{2}{*}{$\begin{array}{l}\text { Tiefe } \\
{[\mathrm{cm}]}\end{array}$} & \multirow{2}{*}{$\begin{array}{c}\mathrm{L}_{\mathrm{d}} \\
{\left[\mathrm{g} / \mathrm{cm}^{3}\right]}\end{array}$} & \multicolumn{3}{|c|}{ Textur $[\mathrm{g} / \mathrm{g} * 100]$} & \multicolumn{4}{|c|}{ Wassergehalte $\left[\mathrm{cm}^{3} / \mathrm{cm}^{3 *} 100\right]$} \\
\hline & & & Sand & Schluff & Ton & $\mathrm{pF}<0$ & $\mathrm{pF} 1,8$ & $\mathrm{pF} 2,5$ & $\mathrm{pF} 4,2$ \\
\hline $\mathrm{L}$ & \multirow{3}{*}{$(-5)-0$} & - & - & - & - & - & - & - & - \\
\hline $\mathrm{O}_{\mathrm{F}}$ & & - & - & - & - & - & - & - & - \\
\hline $\mathrm{O}_{\mathrm{H}}$ & & - & - & - & - & - & - & - & - \\
\hline$A_{h}$ u. $A_{e}$ & $0-5$ & 0,88 & 16 & 69 & 15 & 72,8 & 66,7 & 42,0 & 10,4 \\
\hline $\mathrm{A}_{1}$ & $5-40$ & 1,25 & 9 & 76 & 15 & 47,1 & 30,3 & 24,5 & 7,6 \\
\hline $\mathrm{B}_{\mathrm{t}}$ & $40-140$ & 1,53 & 9 & 69 & 22 & 44,1 & 38,9 & 32,8 & 18,5 \\
\hline $\mathrm{B}_{\mathrm{vt}}$ & $140-160$ & - & 10 & 66 & 27 & - & - & - & - \\
\hline $\mathrm{B}_{\mathrm{v}} \mathrm{C}$ & $160-260$ & - & 5 & 78 & 17 & - & - & - & - \\
\hline $\mathrm{C}$ & $>260$ & - & 10 & 79 & 10 & - & - & - & - \\
\hline
\end{tabular}

\subsection{Entnahme der Bodenproben}

Die Probenahme erfolgte zu verschiedenen Zeitpunkten von April 1997 bis März 1998. Für die Korngrößenanalyse wurden gestörte Proben entnommen. Für die Entnahme ungestörter Proben wurden Stechzylinder und Kubienakästen verwendet. Das Volumen der Stechzylinder zur Erstellung von Retentionskurven und zur Ermittlung der gesättigten Wasserleitfähigkeit sowie der Phasenanteile betrug $100 \mathrm{~cm}^{3}$. Stechzylinder von $250 \mathrm{~cm}^{3}$ wurden für Proben zur Ermittlung der ungesättigten Wasserleitfähigkeit (ku-Kurve) verwendet. Zur Präparierung von Dünnschliffen für die digitale Bildanalyse wurden ungestörte Proben in Kubienakästen der Größe von 4 x 6 x $8 \mathrm{~cm}^{3}$ entnommen.

\subsection{Präparation der Bodenproben für Dünnschliffe}

Zunächst wurden die Proben für einige Tage bei Zimmertemperatur luftgetrocknet, einige Kubienakästen wurden feldfrisch weiterverarbeitet (z. B. der für die Serie Leo9 verwendete, s. u.). Die Proben wurden mit Aceton entwässert, wobei die Kubienakästen mit perforierten Deckeln versehen waren. Nach 10 Tagen wurde das Aceton gegen frisches ausgetauscht, nach weiteren drei bis vier Tagen kann der Entwässerungsvorgang als beendet angesehen werden. Als Gießharz wurde das Polyesterharz Vestopal 160 verwendet, das wie folgt mit einem organischen Lösungsmittel, Härter und Beschleuniger angesetzt wurde (Praktikumsskript Universität Trier)

$\begin{array}{ll}\text { Vestopal 160 } & 800 \mathrm{ml} \\ \text { Styrol } & 200 \mathrm{ml} \\ \text { Cyclohexanonperoxid (Härter) } & 0,6 \mathrm{ml} \\ \text { Cobaltoctat (Beschleuniger) } & 0,3 \mathrm{ml}\end{array}$


Um die lange Aushärtzeit zu beschleunigen, können höhere Mengen Härter und Beschleuniger zugegeben werden, aber immer im Verhältnis 2:1 und nie gleichzeitig (Explosionsgefahr). Die trockenen Proben wurden in Polyethylenbehälter überführt und diese vorsichtig neben der Probe mit dem Polyesterharzgemisch aufgefüllt. Anschließend erfolgte eine Evakuierung im Exsikkator, um eingeschlossene Luftblasen zu entfernen. Während der Aushärtezeit blieben die Proben im Exsikkator. Eine Resttrocknung der oberen Harzschicht erfolgte im Trockenschrank bei $40^{\circ} \mathrm{C}$ über drei bis vier Tage. Die Proben zur Erstellung von Dünnschliffen wurden nach einem vorher festgelegten Schema in Klötzchen gesägt. Die Orientierung der Probe muss während des gesamten Präparationsvorgangs sorgfältig markiert werden, vor allem beim Sägen des Blockes und anschließend der Klötzchen. Eine Seite der Klötzchen wurde mit feiner werdender Körnung (230, 320, 800 Korund) geschliffen und mit demselben Polyesterharz auf einen zuvor gereinigten Objektträger geklebt. Dafür waren Härter und Beschleuniger höher konzentriert: $100 \mathrm{ml}$ Vestopal 160 wurden mit 0,6 ml Cyclohexanonperoxid und 0,3 ml Cobaltoctat angesetzt. Das Klötzchen wurde nahe am Objektträger abgesägt und die Oberseite wie angegeben auf $30 \mu \mathrm{m}$ geschliffen und zusätzlich mit einer Korngröße von 3 $\mu \mathrm{m}$ poliert. Die für die Rasterelektronenmikroskopie verwendeten Schliffe wurden auf 1 $\mu \mathrm{m}$ fein poliert und vor der Installation in die Probenkammer mit Kohlenstoff bedampft..

Abschließend soll erwähnt werden, dass technisch bedingt alle Präparationsschritte mit Wasserkühlung durchgeführt wurden, was die Qualität der Dünnschliffe unseres Erachtens nicht beeinträchtigte. 


\section{$4 \quad$ Methoden}

Während in anderen Disziplinen, besonders in der Biologie und der Medizin, aber auch in den Materialwissenschaften, die digitale Bildanalyse schon seit längerer Zeit als Untersuchungsmethode eingesetzt wird, stellt sie einen recht neuen Ansatz für die Untersuchung der Bodenstruktur dar. Die digitale Bildanalyse bietet den enormen Vorteil, über die Mikromorphologie hinaus (z. B. Bullock et al. 1985, FitzPatrick 1993, Altemüller 1995) die tatsächliche Struktur des Bodens an entsprechend präparierten Proben nicht nur abzubilden, sondern eine Vielzahl von Parametern messen zu können. Hinzu kommt der gegenwärtig rapide wachsende technische Fortschritt, der durch höhere Speicherleistungen und schnellere Prozessoren immer umfangreichere Möglichkeiten bietet, digitale Bilder erzeugen, speichern und verarbeiten zu können.

Für die hier bearbeitete Fragestellung der Messung fraktaler Eigenschaften der Bodenstruktur wurde die Grenzfläche zwischen Partikeln und Poren als Untersuchungsgegenstand gewählt. Ihr kommt für die Charakterisierung des Porenraums als strukturgebendem Faktor eine besondere Bedeutung zu. Das gilt für die hydraulischen Eigenschaften des porösen Mediums und die Beschreibung der inneren Oberfläche als dem Ort für biotische und abiotische Reaktionsprozesse. Diese Überlegungen werden durch die Arbeiten von Pape et al. (1987), Bartoli et al. (1991) und Perrier et al. (1999) bestätigt. Germann \& Di Pietro (1999) und Germann (2000) benutzen die Kontaktlänge des mobilen Wassers pro Flächeneinheit Boden als Parameter für die Simulation des Wassertransports nach dem Modell der kinematischen Wellen.

Bei den Untersuchungen der Bodenstruktur standen verschiedene methodische Schwerpunkte im Vordergrund, die sich in folgende Arbeitsschritte gliedern lassen:

Präparation und Bildgewinnung

Software-Entwicklung für die digitale Bildanalyse

Auswertung der bildanalytisch erhobenen Daten

Klassische bodenphysikalische Untersuchungen an Stechzylinderproben

Die Präparation wurde bereits in Kapitel 3.3 „Präparation der Bodenproben für Dünn- und Anschliffe" dargestellt. Der Bildgewinnung kam im Rahmen dieser Arbeit eine besondere Bedeutung zu, gute Ergebnisse waren ausschließlich durch eine hohe Qualität des Eingangsbildes erreichbar. Jede Verarbeitung des digitalen Bildes im Sinne einer Bildverbesserung hätte die Veränderung des Untersuchungsabbildes - der Porenraumgrenzlinie - nach sich gezogen. Die Software für die bildanalytische Messung der fraktalen Dimension wurde von einer älteren Plattform (IBAS ${ }^{\circledR}$ 2.0, Kontron, Eching; Eins 1995, Eins \& Stiller 1995) für das Nachfolge-System KS400 ${ }^{\circledR}$ (ZeissVision, Jena) umgeschrieben, aktualisiert und verbessert. Die Porengrößenverteilung wurde mit dem Opening-Verfahren (Serra 1982) gemessen, das von Vogel (1996) zur Messung der Porengrößenverteilung im dreidimensionalen Raum verwendet wurde. Bei der Auswertung des umfangreichen Datenmaterials konnte kaum auf vorhandene Anleitungen zurückgegriffen werden, da nur zu einzelnen Problemstellungen wissenschaftliche Publikationen existieren. So wurden teilweise eigene Methoden entwickelt (s. Kap. 5). Bei den klassischen bodenphysikalischen Untersuchungen handelt es sich bis auf die Messung der ungesättigten Wasserleitfähigkeit an Einzelaggregaten um etablierte Methoden. 


\subsection{Optimierung der Bildqualität}

Dieser Teil ist essentiell für die digitale Bildanalyse. Bereits die sorgfältige Präparation wenngleich zeitintensiv - ist Voraussetzung für eine optimale Bildqualität, die wiederum Voraussetzung für die Segmentierbarkeit in Porenraum und Matrix ist. Die Erarbeitung geeigneter Bildaufnahmetechniken nahm viel Zeit in Anspruch, beginnend mit der sorgfältigen Probennahme im Gelände, dem Probentransport, der Kunstharz-Einbettung und schließlich der Herstellung physikalischer Schnitte/Schliffe für die verwendeten mikroskopischen Techniken. In Abb. 4.1 ist die erarbeitete Vorgehensweise als Fließdiagramm dargestellt.

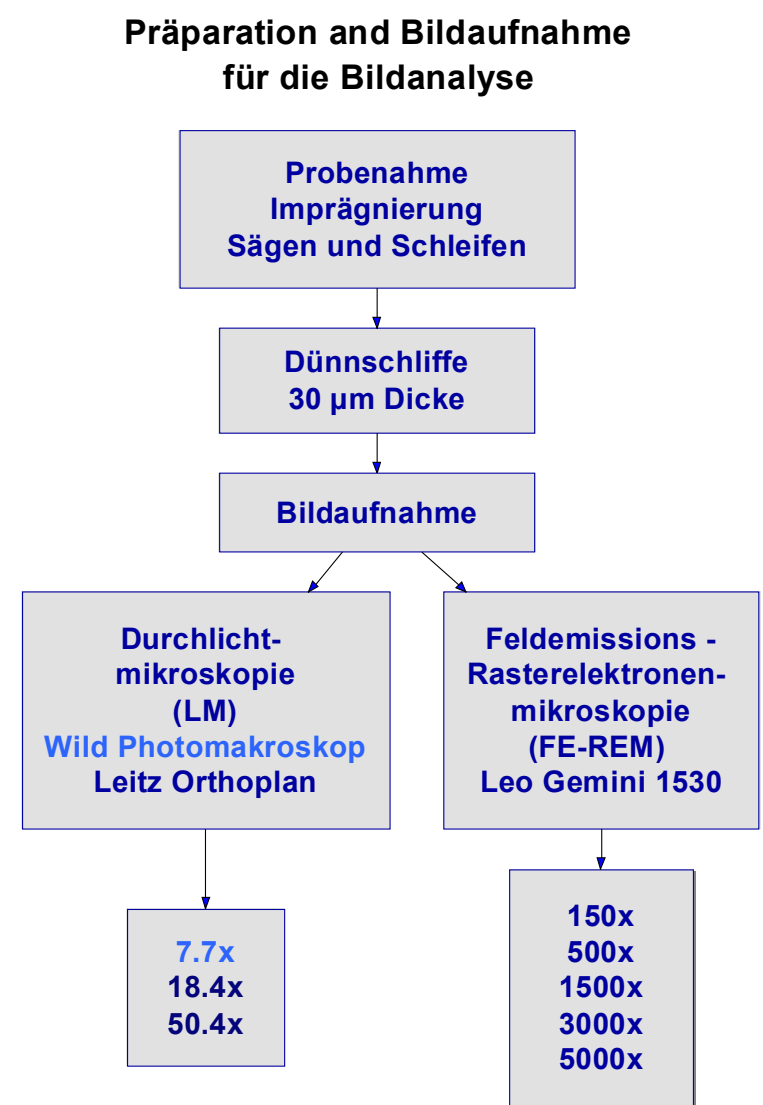

Abb. 4.1: Vorbereitende Arbeiten für die digitale Bildanalyse einschließlich der verwendeten Mikroskopietechniken und Vergrößerungsstufen.

Um die Problematik zu verdeutlichen, sind in Abb. 4.2 mit den verschiedenen mikroskopischen Verfahren Durchlichtmikroskopie (LM) und FeldemissionsRasterelektronenmikroskopie (FE-REM) gewonnene digitale Bilder und die Histogramme ihrer Grauwertverteilungen dargestellt. Die lichtmikroskopisch aufgenommenen Bilder weisen in Bezug auf die Differenzierung in Poren und Matrix einen nicht eindeutigen Grauwertkontrast auf und lassen sich schlecht segmentieren. 

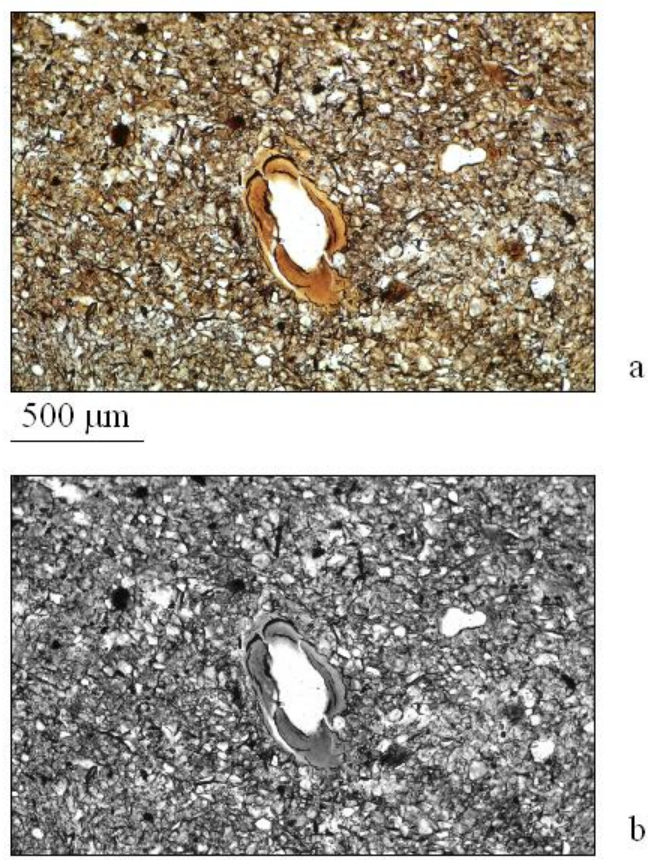

b

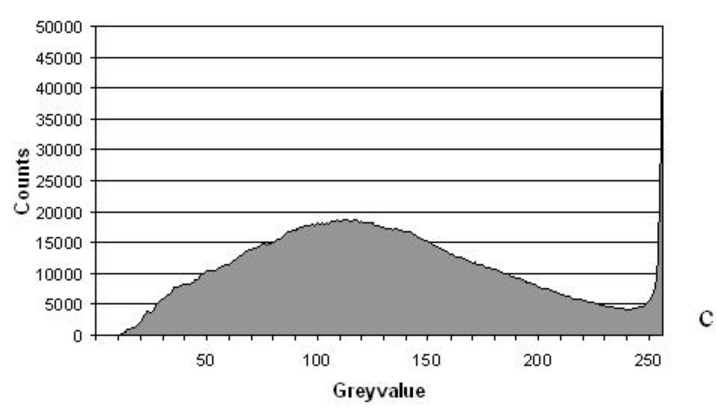

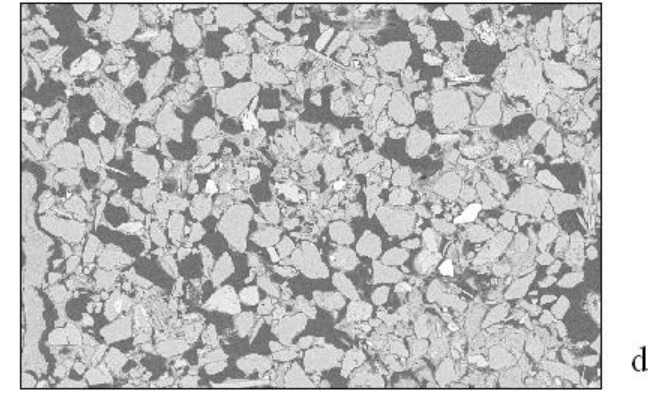

$100 \mu \mathrm{m}$
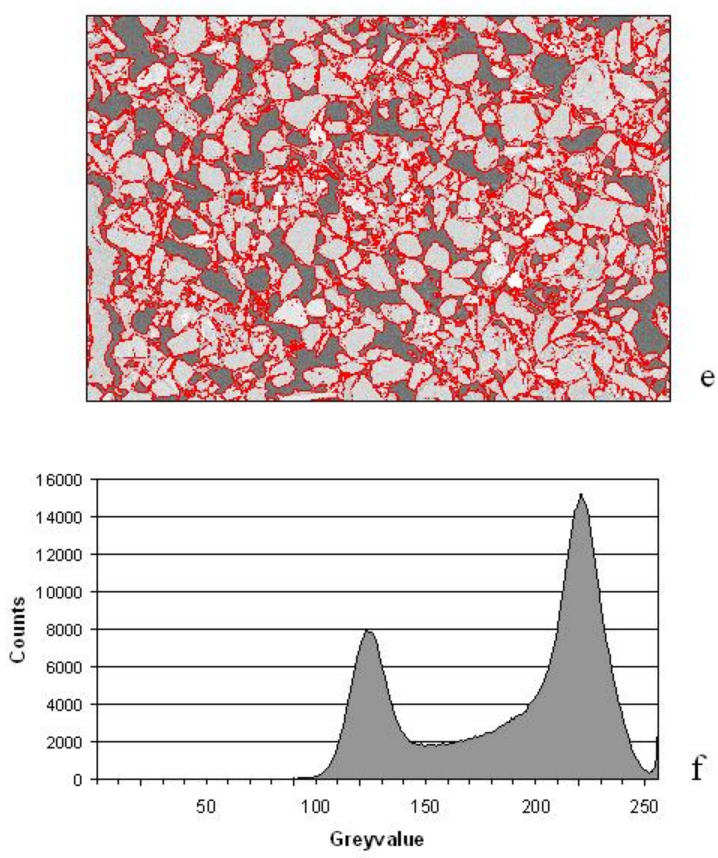

Abb. 4.2: Aufnahmen von Bodendünnschliffen mit den entsprechenden Grauwertverteilungen. (a) Farbild (RGB), lichtmikroskopische Aufnahme im Hellfeld; (b) Graubild Transformation von (a); (c) Grauwerthistogramm von (b) ohne erkennbare Segmentierungsschwelle; (d) FE-REM Aufnahme, der mit Gießharz ausgegossene Porenraum erscheint dunkel; (e) Konturen der Grenzlinie Pore/Matrix über dem Originalbild (d); (f) Grauwerthistogramm von (d) mit deutlich unterscheidbaren Peaks für Poren (links) und Matrix (rechts).

Im Durchlicht zeigten Quarze und Poren bzw. das Einbettungsharz eine gleich hohe Luminanz und erforderten zur Unterscheidung den Einsatz polarisierten Lichtes. Die endliche Schnittdicke der Dünnschliffe, die mit $30 \mu \mathrm{m}$ im Bereich des Durchmessers der Schluffkörner lag, limitierte die Segmentierbarkeit der Poren durch Anschnitt- und Überlagerungsphänomene. Die breit gestreuten Grauwertverteilungen der Poren zeigten keine Ansatzpunkte für eine objektive und erfassende Grauschwelle für die Porensegmentierung (s. Abb. 4.2 c). Deshalb kam als Routinemethode die Rasterelektronenmikroskopie zum Einsatz, die eine scharfe Abbildung der Porenkontur bietet (s. Abb. $4.2 \mathrm{~d}-\mathrm{f})$.

Im Fein- und Mittelporenbereich war die Elektronenmikroskopie unverzichtbar, der Bereich der weiten Grobporen wurde jedoch wegen des limitierten Bildausschnittes nur unzureichend erfasst. Nennenswert geringere Vergrößerungen als 150x können im REM nicht ohne Randverzerrungen abgebildet werden. Daher musste für Übersichtsaufnahmen trotz der oben genannten Einschränkungen mit Polarisations-Lichtmikroskopie gearbeitet werden. 


\subsection{Mikroskopiertechniken}

Das menschliche Auge hat eine Auflösung von bis zu 0, 2 mm. Mit einem Lichtmikroskop können bei der Verwendung von Immersionsobjektiven $0,2 \mu \mathrm{m}$ und mit einem Rasterelektonenmikroskop maximal 0,2 nm erreicht werden. So ergibt sich für diese drei optischen Systeme eine Verbesserung der Auflösung um jeweils den Faktor 1000 (Gerlach 1995).

\subsubsection{Lichtmikroskopie}

Ein Lichtmikroskop ist ein aus Linsensystemen (Kondensor, Objektiv, Okular) bestehendes optisches Vergrößerungsgerät. Die Kombination mehrerer Linsen dient auch der Korrektur von Abbildungsfehlern. Der Kondensor wirkt wie ein umgekehrtes Objektiv und wird zur Beleuchtung benutzt. Das Objektiv erzeugt ein vergrößertes, seitenverkehrtes und reelles Bild des Untersuchungsgegenstandes. Das erhaltene Bild wird mit Hilfe des Okulars betrachtet, das wie eine Lupe arbeitet und das Bild noch einmal vergrößert, aber nicht höher auflöst. Die Gesamtvergrößerung berechnet sich aus dem Produkt der Einzelvergrößerungen.

Viele Minerale in Böden sind doppelbrechend, d. h., in ihnen kann das Licht nur in zwei Richtungen senkrecht zur Fortpflanzungsrichtung schwingen, der Brechungsindex ist für jede Richtung verschieden. In den Geowissenschaften werden durch die PolarisationsMikroskopie Minerale bestimmt, in der vorliegenden Arbeit wird sie zur Erhöhung des Kontrastes zwischen dem Einbettungsharz und Quarzkörnern benutzt. Dafür werden Polarisator und Analysator in Kreuzstellung gebracht. Der Polarisator befindet sich unterhalb des Kondensors und lässt nur Lichtwellen durch, die in einer Raumebene schwingen. Der Analysator wird zwischen Objektiv und Okular in den Tubus eingeführt und dient der Analyse der Veränderungen der Schwingungsebene, die die polarisierten Lichtwellen im Präparat erfahren haben. Trifft linear polarisiertes Licht auf ein doppelbrechendes Medium, erfährt es keine Veränderung, wenn die Schwingungsrichtung einer der beiden Durchlassrichtungen entspricht. Ist dies nicht der Fall, wird das linear polarisierte Licht auf beide Schwingungsrichtung aufgeteilt. Beide interferieren, und durch die vier möglichen Gangunterschiede erscheint ein doppelbrechendes Objekt beim Drehen des Mikroskoptisches um $360^{\circ}$ viermal hell und viermal dunkel.

\subsubsection{Feldemissions-Rasterelektronenmikroskopie}

Die hohe Auflösung in der Rasterelektronenmikroskopie wird durch die Bestrahlung mit Elektronen statt mit einer Lichtquelle erreicht. Daneben zeichnet sie sich durch eine gegenüber der Lichtmikroskopie wesentlich größere Schärfentiefe aus. In einer Kathode wird ein Elektronenstrahl erzeugt, durch magnetische Linsen gebündelt und über die Probenoberfläche gerastert. Das gesamte System arbeitet im Vakuum, weshalb ausgasende Proben nicht untersucht werden können. Die beschleunigten Elektronen erfahren vielfältige Wechselwirkungen an den Atomen der untersuchten Probe. Einen Überblick vermittelt die Abb. 4.3. Elektronen können aus der Probenoberfläche abgelöst oder aus tiefer gelegenen Bereichen zurückgestreut werden. Mit speziellen Detektoren, die sich wie das Präparat in 
der Probenkammer befinden, werden die emittierten Elektronen registriert und aus dem Rastermuster das Bild konstruiert.

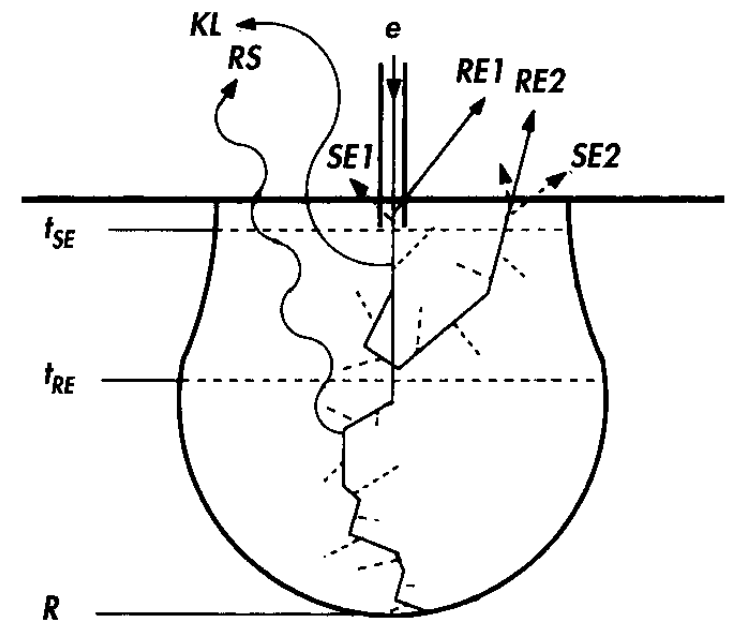

Abb. 4.3: Bei der Bestrahlung der Probe mit dem Elektronenstrahl kommt es auf Grund von elastischen und unelastischen Streuprozessen zur Ausbildung eines birnenförmigen Anregungsvolumens. Seine Tiefenausdehnung entspricht der Elektronenreichweite R. Im Anregungsvolumen entstehen Sekundärelektronen (SE1 und SE2), Röntgenstrahlung (RS) und Kathodoluminiszens-Strahlung (KL). Ein Teil der rückgestreuten Elektronen kann als RE1 und RE2 die Probe aus einer maximalen Austrittstiefe $t_{\mathrm{RE}}$ verlassen. Die Austrittstiefe für Sekundärelektronen ( $t_{\mathrm{SE}}$ ) ist wesentlich geringer. Aus Reichelt (1995), verändert.

Das verwendete FE-REM (Leo Gemini $1530^{\circledR}$, Zeiss, Oberkochen) arbeitet nach dem Prinzip der Niedervolt-Elektronenmikroskopie und verfügt über eine Kaltfeld-Emissionskathode, d.h. der Austritt von Elektronen erfolgt durch die Einwirkung eines elektrischen Feldes bei Zimmertemperatur. Verwendet wird eine Schottky Kathode aus Wolfram und Zirkon. Durch das elektrische Feld wird die Potentialschwelle für den Austritt der Elektronen herabgesetzt (Schmidt 1994). Das Verfahren ermöglicht eine sehr hohe Elektronendichte im Primärstrahl bei einer geringen Schwankungsbreite der Elektronenenergien $(\Delta \mathrm{E} \approx 0,2 \mathrm{eV})$. Dadurch wird der chromatische Fehler, der mit der Energiedifferenz der Elektronen zunimmt (unterschiedliche Geschwindigkeiten der Elektronen führen $\mathrm{zu}$ Brennweitendifferenzen) und eine Strahlaufweitung in der Fokusebene verursacht, sehr gering. So wird eine hohe Auflösung $(\leq 1 \mathrm{~nm})$ bei niedriger Beschleunigung erreicht. Dadurch wird eine geringe Eindringtiefe ermöglicht, die zu einer deutlichen Abbildung der Oberfläche eines Präparates ohne Überlagerung durch zusätzliche Tiefeninformation führt. Ein weiterer großer Vorteil der Verwendung der Niedervolt-Elektronenmikroskopie ist, nichtleitende Proben auch ohne die Aufbringung einer elektrisch leitenden Schicht untersuchen zu können. Zwischen einigen $100 \mathrm{eV}$ und $1000 \mathrm{eV}$ existiert ein optimaler Bereich, in dem mehr Elektronen aus der Probe austreten als eingestrahlt werden, so dass sich keine Oberflächenladung ausbilden kann. Bei Verwendung einer Kaltfeld-Elektrode muss, um die Schädigung der Kathodenspitze durch Ionisation gering $\mathrm{zu}$ halten und eine möglichst stabile Elektronenemission zu gewährleisten, mit einem Hochvakuum von etwa $10^{-7}$ Pa gearbeitet werden (Schmidt 1994).

Die austretenden Elektronen können im wesentlichen in Rückstreu- und Sekundärelektronen unterteilt werden (s. Abb. 4.3). Bei den Rückstreuelektronen handelt es sich um aus der Oberfläche der Probe austretende Primärelektronen. Ihre Reichweite verhält sich umgekehrt proportional zur Ordnungszahl $\mathrm{Z}$ des bestrahlten Atoms, die 
Auflösung entspricht etwa der halben Reichweite der Elektronen in der Probe. Rückstreuelektronen sind sehr gut geeignet, um einen Materialkontrast von Substanzen mit deutlich unterschiedlicher Ordnungszahl abzubilden. Sekundärelektronen (SE) werden von Primärelektronen (SE1) und Rückstreuelektronen (SE2) auf der Oberfläche der Probe durch Ionisation aus dem Probenmaterial herausgelöst. Ihre Austrittstiefe liegt im Nanometerbereich und entspricht ungefähr der Auflösung der SE. Im Vergleich zu Rückstreuelektronen ist der Energiegehalt der Sekundärelektronen wesentlich geringer und ihre Auflösung wesentlich feiner. Mit Sekundärelektronen können Oberflächentopographien sehr gut, ein Materialkontrast dagegen weniger gut abgebildet werden, da das Signal nur für kleine $\mathrm{Z}$ eine lineare Abhängigkeit zeigt. Auf die ebenfalls auftretende Kathodolumineszens-Strahlung und die Röntgenstrahlung (s. Abb. 4.3) soll hier nicht eingegangen werden.

\subsection{Anwendung der verschiedenen Mikroskopiertechniken}

\subsubsection{Lichtmikroskopie}

Zunächst wurden lichtmikroskopische Aufnahmen der Dünnschliffe mit einem Axiophot ${ }^{\circledR}$, Zeiss, Oberkochen (s. Abb. 4.4) und einem Orthoplan ${ }^{\circledR}$, Leitz, Wetzlar (s. Abb. 4.5) erstellt. Die Bilder waren zur visuellen Darstellung und qualitativen Untersuchung (z. B. zur Charakterisierung der Mineralienzusammensetzung) sehr gut, hinsichtlich eines bildanalytischen Messansatzes jedoch weniger gut geeignet (s. Abb. 4.2 a,b). Die komplexe Struktur erlaubte keine eindeutige Segmentierung in Poren und Matrix (s. das Histogramm in Abb. $4.2 \mathrm{c}$ ). Die erstellten Bilder wurden nur ansatzweise bildanalytisch weiterverarbeitet.

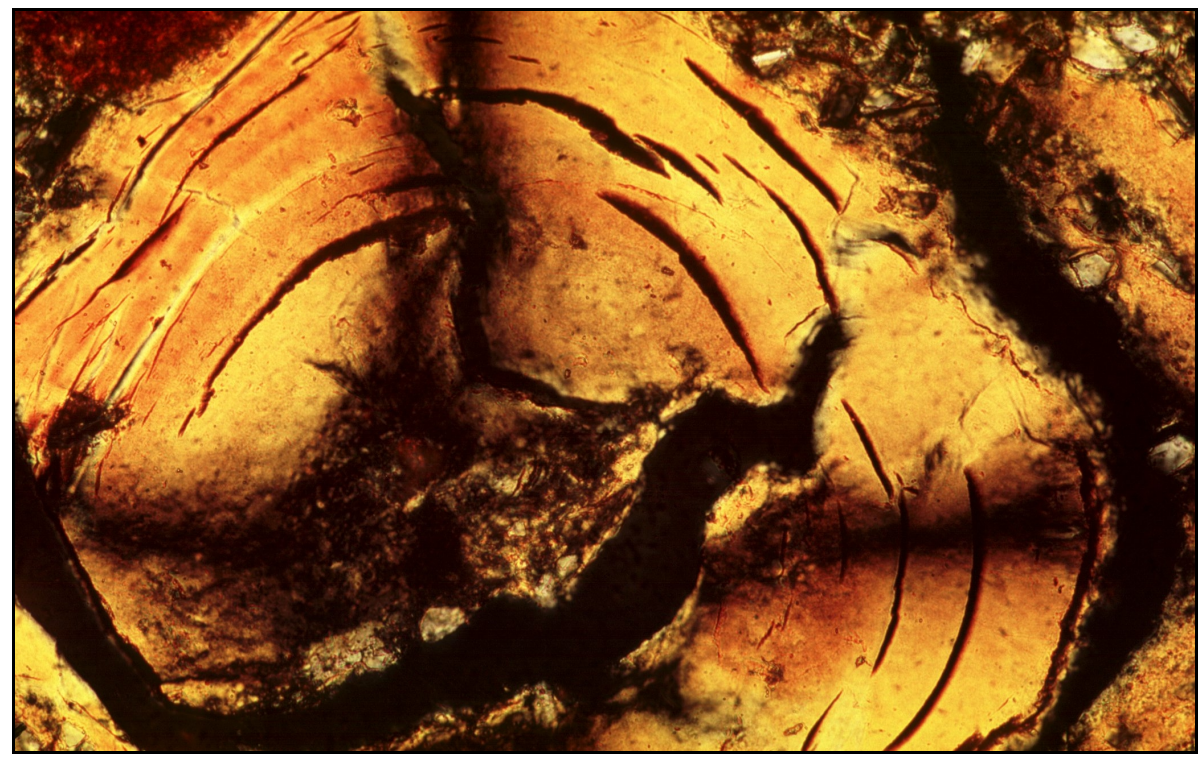

Abb. 4.4: Aufnahme einer Tonablagerung am Zeiss Axiophot, Objektiv 2,5, Okular 10, gekreuzte Polarisatoren, die schmale Bildkante misst 0,44 mm. Die Lamellenstruktur der Tonablagerung ist deutlich erkennbar, es finden sich einzelne Schluffkörner. Die Ränder des Risses auf der rechten Bildseite sind ausgefranst, der Riss scheint präparationsbedingt entstanden zu sein. 

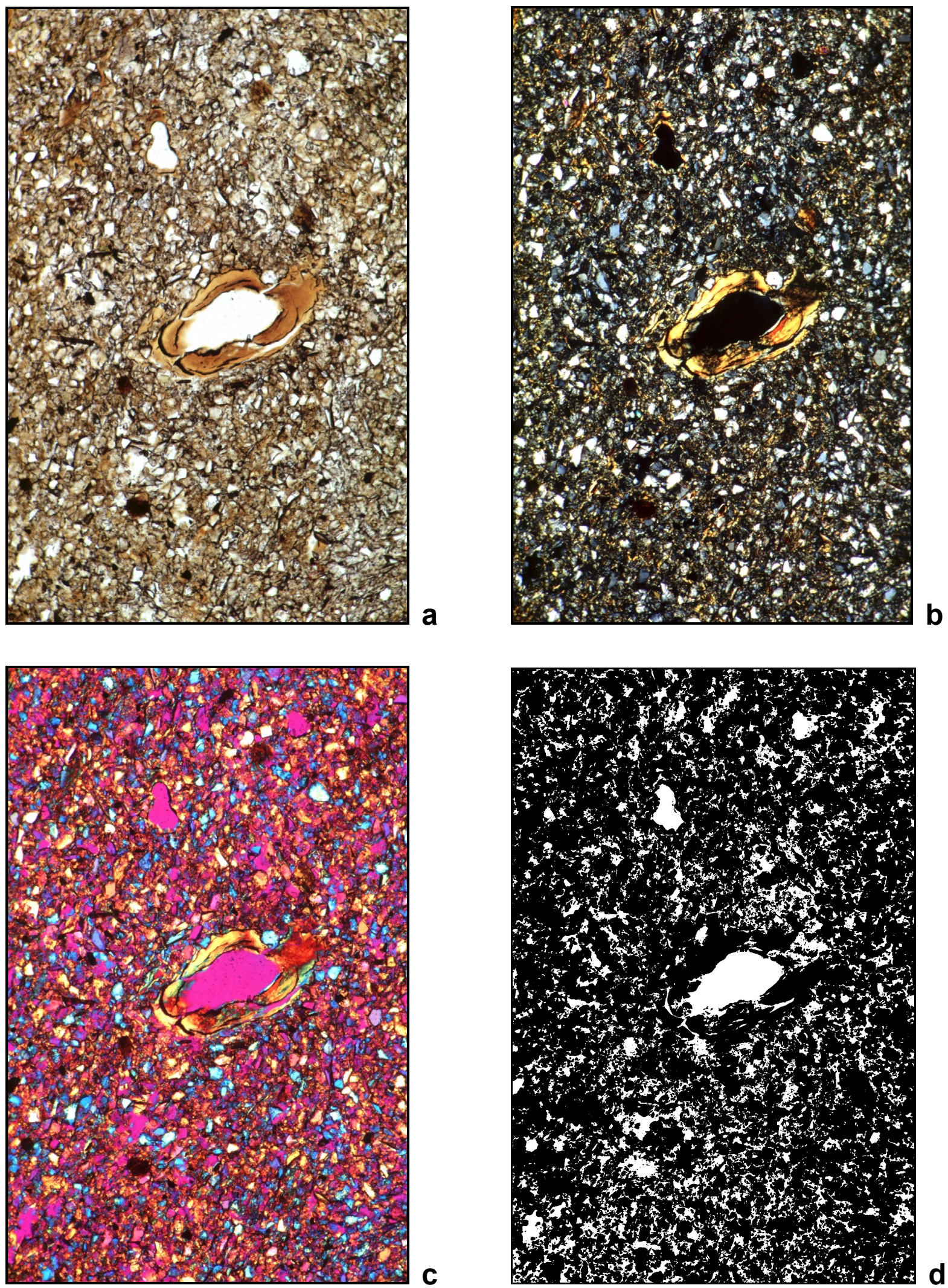

Abb. 4.5: Aufnahmen am Leitz Orthoplan, Objektiv 6,3x, Tubusfaktor 8, die schmale Bildseite misst 1,50 mm. (a) Im Durchlicht, deutlich erkennbar sind eine mit Tonkutanen ausgekleidete Makropore innerhalb von Schluffkörnern sowie sehr dunkle Mangankonkretionen; (b) zusätzlich mit gekreuzten Polarisatoren, Poren erscheinen schwarz und die Quarzkörner weiß bis hellblau; (c) mit gekreuzten Polarisatoren und Quarz Rot I Kompensator, verschiedene Minerale können detektiert werden, der Porenraum erscheint magenta; (d) um im Binärbild von (b) einen Porenanteil (weiß) von $40 \%$ zu erhalten muss deutlich übersegmentiert werden. 

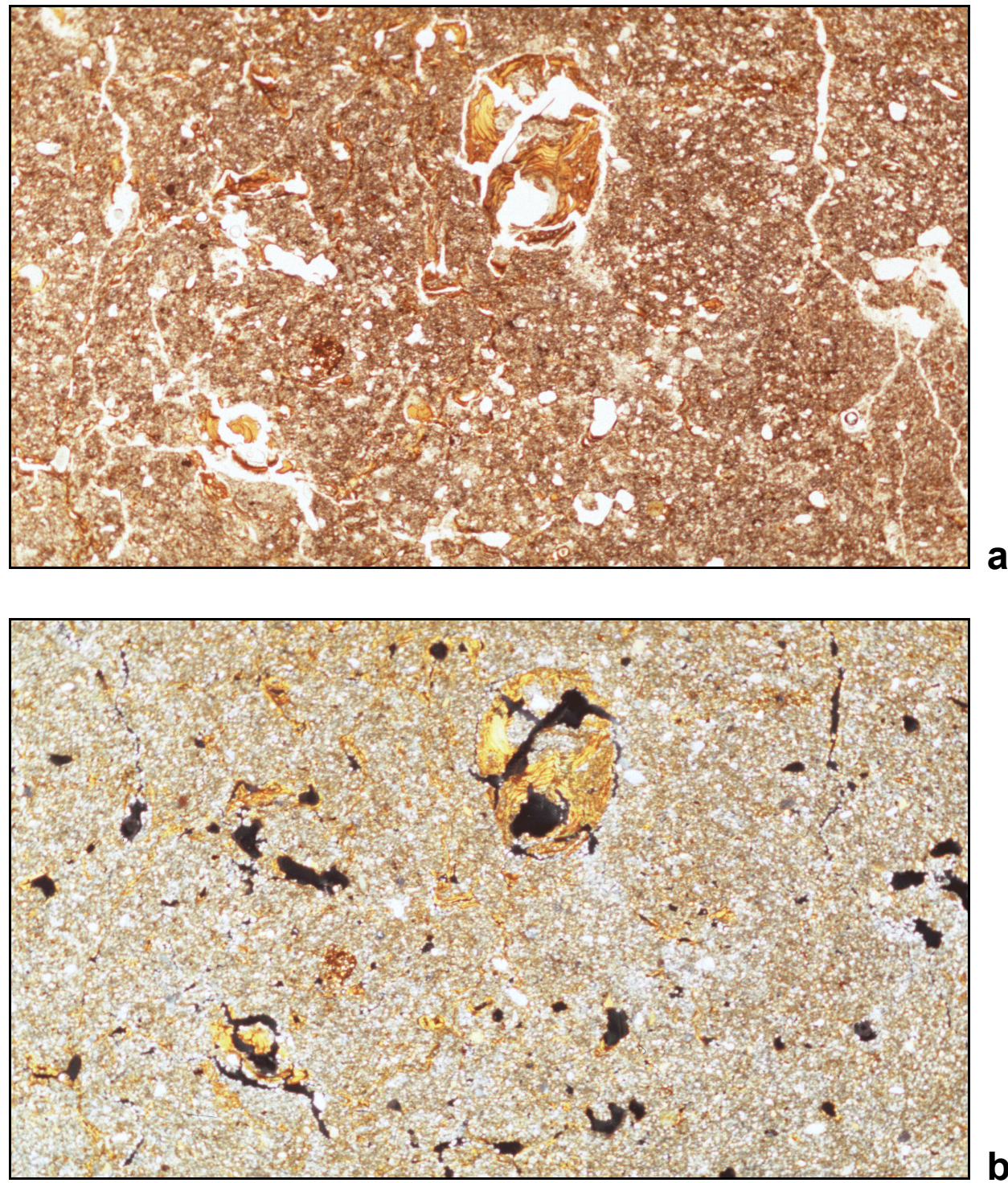

b

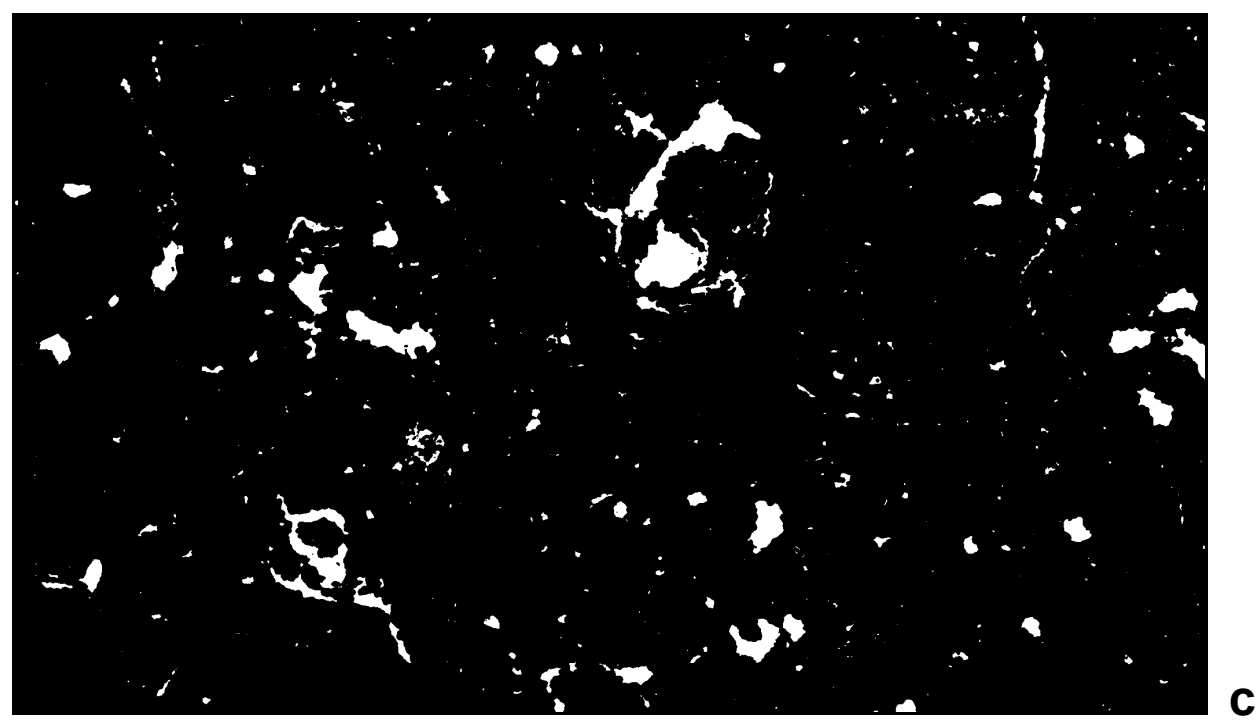

Abb. 4.6: Durchlichtaufnahmen des Dünnschliffes Wild7 (vertikale Ausrichtung) mit dem Wild-Photomakroskop, Objektiv 6.3. (a) im Hellfeld, (b) mit polarisiertem Licht, (c) nach Grauwertsegmentierung erhaltenes Binärbild von (b). Die Bildgröße beträgt rund 16,0 x 9,3 mm. 
Übersichtsaufnahmen wurden im Durchlicht und polarisiertem Licht (Photomakroskop M400 ${ }^{\circledR},(1,25 \mathrm{x})$ und Objektiv 6,3 Wild, St. Gallis) erstellt (s. Abb. 4.6). Mit Polarisator und Analysator in Kreuzstellung konnte eindeutig zwischen dunklen Poren und hellen Quarzkörnern unterschieden werden. Die Bildgröße deckte mit ca. 9,3 mm x $16 \mathrm{~mm}$ fast das gesamte Präparat ab. Bei der verwendeten Auflösung des Diascanners von 1500 dpi betrug die Auflösung 7,76 $\mu \mathrm{m} /$ Pixel. Es wurden 17 Aufnahmen, die von Dünnschliffen eines Kubiena Kastens stammen, detailliert ausgewertet. Dabei handelte es sich um 10 vertikal und 7 horizontal ausgerichtete Schliffe, von denen je einer pro Ausrichtung für die REM-Aufnahmen (Datensätze Leo7 und Leo8) verwendet wurde.

\subsubsection{Feldemissions-Rasterelektronenmikroskopie}

Vor der Installation in die Vakuumkammer des FE-REM wurden die feinpolierten Dünnschliffe mit Kohlenstoff bedampft. Die Bedampfung lässt die Elektronen abfließen und verhindert so Überstrahlungseffekte. Für die vorliegende Anwendung erwies sich eine Kombination aus 3/4 Rückstreu- und $1 / 4$ Sekundärelektronen für die Detektierung als optimal (Abb. $4.2 \mathrm{~d}$ und 4.7), was mit dem deutlichen Unterschied der Z-Zahlen des Einbettungsmediums (vor allem Kohlenstoff, $Z_{C}=6$ ) und der Matrix (für Silizium ist $Z_{S i}=14$ ) begründet werden kann. Die Histogramme der Grauwerte der so erhaltenen digitalen Bilder weisen eine klare zweigipflige Verteilung auf, was gute Voraussetzungen für die Segmentierbarkeit bietet (Abb. 4.2 e und f). Gearbeitet wurde bei einer Anregungsspannung von $18 \mathrm{keV}$, einem Arbeitsabstand von 8 bis $9 \mathrm{~mm}$ und einer Apertur der Feldemissionskathode von 30 bzw. $60 \mu \mathrm{m}$. Bei diesen Einstellungen beträgt die Eindringtiefe der Primärelektronen 1 bis $2 \mu \mathrm{m}$.

Tab. 4.1: Vergrößerungsstufen, Auflösungen und Bildgrößen der verschiedenen Mikrokopietechniken. Die Angaben für das LEO GEMINI sind relativ, sie beziehen sich auf den jeweils angeschlossenen Drucker. Die Auflösungen wurden direkt an dem sich auf jedem Bild befindenden Skalierungsbalken gemessen. Es wurde entweder die 1200x oder die 1500x Vergrößerung gewählt.

\begin{tabular}{|c|c|c|c|c|c|}
\hline Vergrößerung & & & & & \\
\hline $\begin{array}{l}\text { Auflösung } \\
\text { [ } \mu \mathrm{m} / \text { Pixel] }\end{array}$ & Wild M400 & Orthoplan & Orthoplan & GEU & GEMINI \\
\hline $\begin{array}{l}\text { Bildgröße } \\
\text { [Pixel · Pixel] }\end{array}$ & $2060 \times 1200$ & $2060 \times 1340$ & $2060 \times 1340$ & $1024 \times 768$ & $2048 \times 1536$ \\
\hline Objektiv $6,3 \mathrm{x}$ & 7,763 & & & & \\
\hline $\begin{array}{l}\text { Objektiv } 2,5 \mathrm{x} \\
\text { Tubusfaktor } 8\end{array}$ & & 2,753 & & & \\
\hline $\begin{array}{l}\text { Objektiv } 6,3 \mathrm{x} \\
\text { Tubusfaktor } 8\end{array}$ & & & 1,157 & & \\
\hline $150 x$ & & & & 2,500 & 0,87 \\
\hline $500 x$ & & & & 0,746 & 0,26 \\
\hline $1200 x$ & & & & 0,213 & \\
\hline $1500 x$ & & & & 0,172 & 0,087 \\
\hline 3000x & & & & 0,122 & \\
\hline $5000 x$ & & & & 0,052 & 0,026 \\
\hline
\end{tabular}



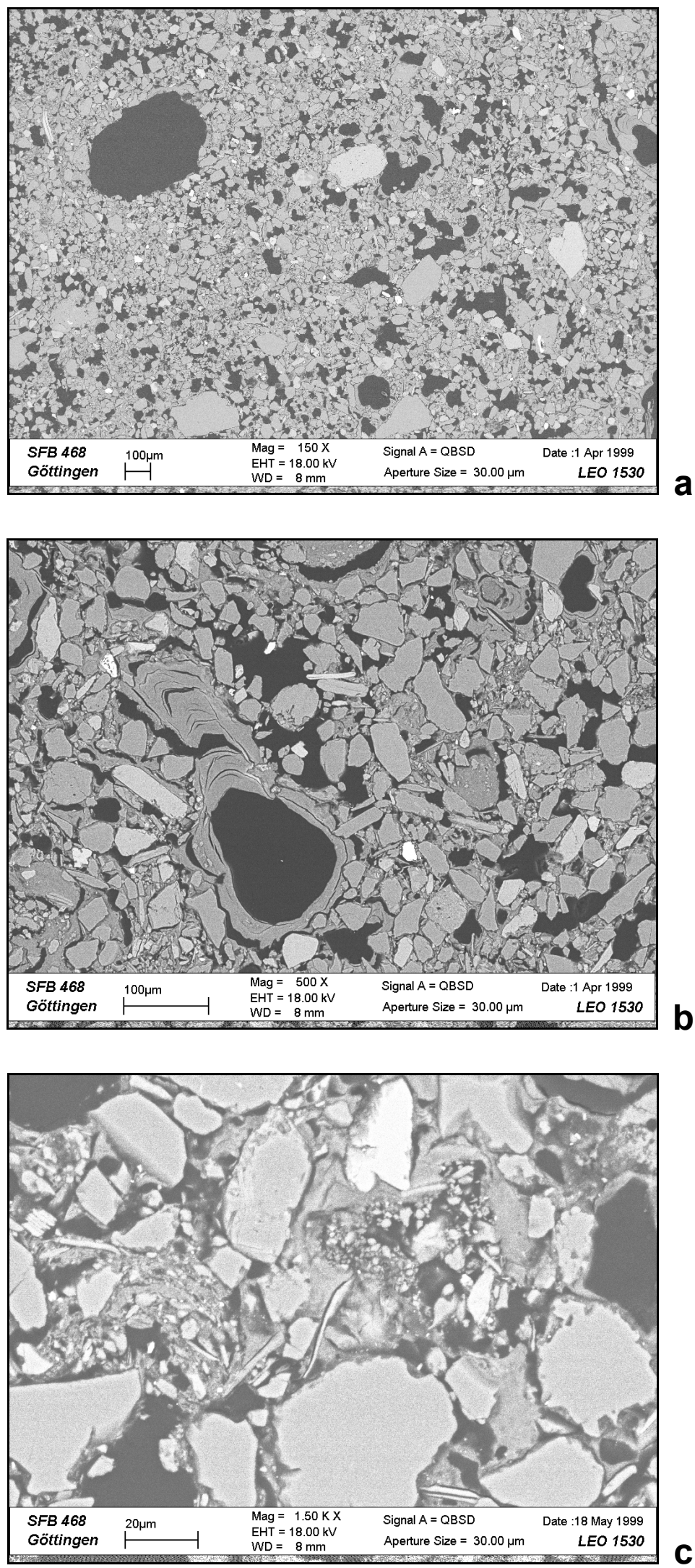

Abb. 4.7: Beispielhafte Darstellung von FE-REM Bildern der Serie Leo8 mit den verwendeten Vergrößerungs-stufen (s. Tab. 4.1). In der Legende sind neben dem Maßstabsbalken die Vergrößerung (Mag), die Anregungsspannung (EHT), der Arbeitsabstand (WD) und die Apertur angegeben. „Signal A“ bezieht sich auf die verwendeten Elektronendetektoren. Während in (a) und (b) einzelne Poren mit und ohne Toncutane erkennbar sind, erscheinen bei den höheren Vergrößerungsstufen zunehmend einzelne Partikel (c). 

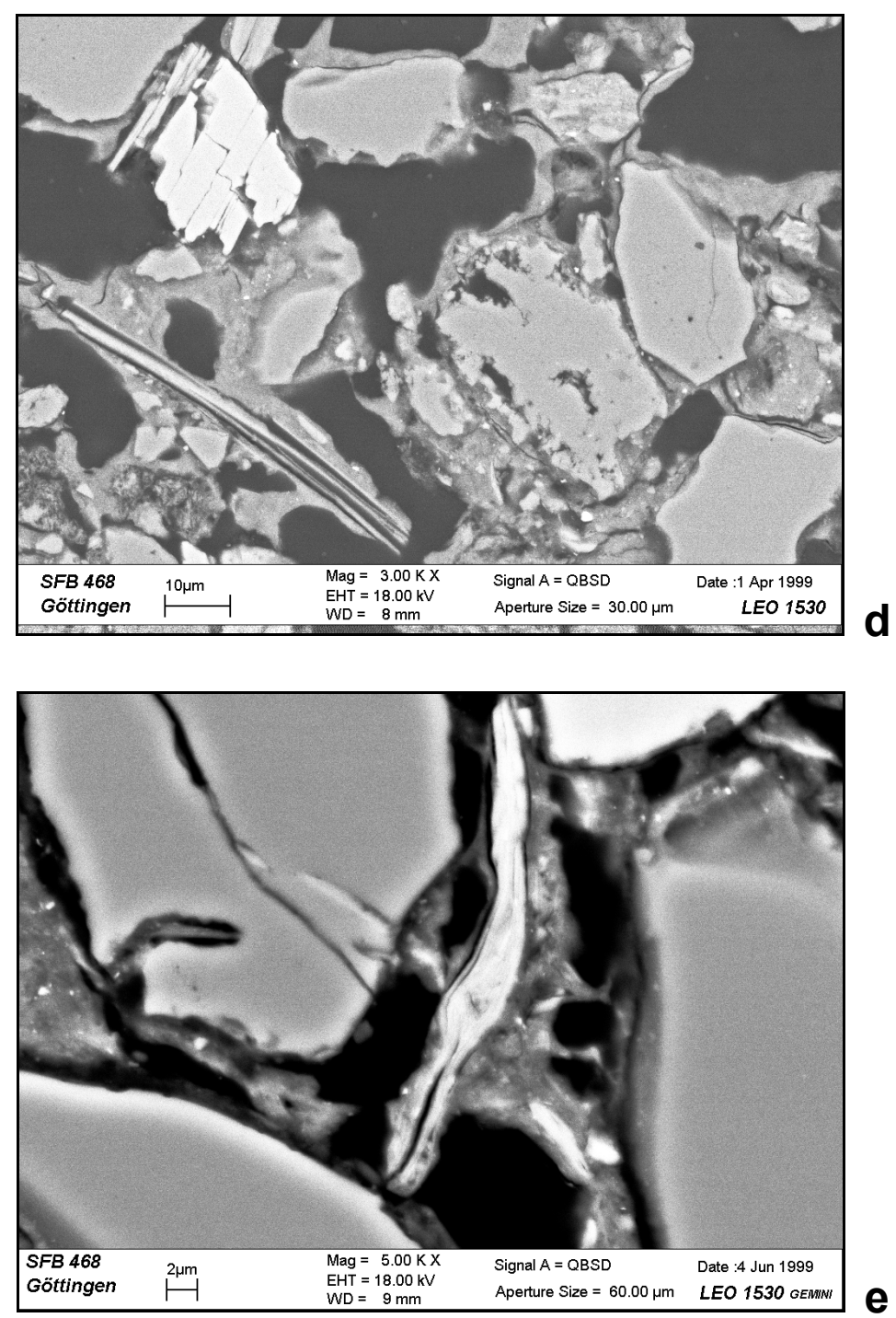

Abb. 4.7: Beispielhafte Darstellung von FE-REM Bildern der Serie Leo8. In den mit höheren Vergrößerungsstufen aufgenommenen Bildern (d und e) wird die feine, tonhaltige Matrix erkennbar, die durch Verkittung Brücken zwischen den einzelnen Körnern bildet. Bei den großen, glatt durchgeschnittenen Körnern handelt es sich um die Schlufffraktion. Minerale, die Schwermetalle enthalten, erscheinen auf Grund ihrer Ordnungszahl sehr hell.

Die am REM erstellten Bildserien wurden an je einem Dünnschliff aufgenommen. Es wurden fünf verschiedene Vergrößerungsstufen verwendet (s. Tab. 4.1 und Abb. 4.7) und jeweils 20 Bilder erstellt. Für die 150x Vergrößerungen wird damit das gesamte Präparat abgerastert. Die Serie Leo9 wurde mit einer doppelt so hohen Auflösung und vier statt fünf Vergrößerungsstufen angefertigt (s. Tab. 4.1). Drei Serien wurden detailliert ausgewertet, dabei handelt es sich um einen vertikal (Serie Leo7, s. Abb. 4.6) und zwei horizontal (Serien Leo8 und Leo9, s. Abb. A1 und A2) ausgerichtete Dünnschliffe. 


\subsection{Grundlagen der digitalen Bildanalyse}

Im Gegensatz zu Geographischen Informationssystemen, die auf Vektor- und Rasterbasis arbeiten können, arbeiten bildanalytische Systeme im Allgemeinen pixelbasiert. Die ursprüngliche Bildgröße von 512 x 512 Pixeln (oder Bildpunkten) entstammt der europäischen Videonorm, da in den Anfangszeiten der digitalen Bildverarbeitung Fernsehkameras zum Bildeinzug verwendet wurden. Die Form eines Pixels ist heutzutage üblicherweise quadratisch, wobei auch drei- oder sechseckige Pixel denkbar wären. Jedem Pixel ist ein Grauwert zugeordnet, der einen Wert zwischen 0 (schwarz) und 255 (weiß) annehmen kann. Das menschliche Auge löst nur ca. 60 Graustufen auf. Bei einem Graubild benötigen die 256 möglichen Grauwerte für jedes Pixel 8 Bit = 1 Byte Speicherbedarf. Bei einem Farbbild ist jedem der drei Farbkanäle Rot, Blau und Grün ein eigener Grauwert zugeordnet, so dass der Speicherbedarf pro Pixel 3 Byte beträgt. Für ein 512 x 512 Pixel großes Bild ergibt sich so ein Speicherbedarf von ca. 262 bzw. 786 Kilobyte (KB) für ein Grau- bzw. Farbbild.

\subsubsection{Bildverarbeitung und Bildanalyse}

Die in der Überschrift genannten Begriffe sind nicht eindeutig festgelegt, z. B. sprechen Bässmann und Besslich (1991) von Bildbearbeitung und Bildauswertung. Eine sehr gute Einführung in die Thematik findet sich in Eins \& Stiller (1995). Als wesentlichen Unterschied arbeiten die Autoren heraus, dass die Bildverarbeitung mit dem Einsatz elektronischer Mittel aus einem Ausgangsbild ein Bild mit gezielt veränderten Eigenschaften erzeugt, während am Ende der Bildanalyse ein Ergebnis im Sinne von Zahlen oder einer Klassifizierung steht. Bei der Bildanalyse findet keine Veränderung der Grauwerte der einzelnen Pixel statt, sondern es wird eine bestimmte Bildinformation gemessen. Im Grunde genommen gehört die Erstellung eines Grauwerthistogrammes (s. Abb. 4.2 c,f) bereits zur Bildanalyse. Das Histogramm zeigt die distributive Verteilung der 256 Grauwerte des gesamten Bildes und bietet so eine leicht $\mathrm{zu}$ messende, zentrale Bildinformation. Mit den modernen Geräten gehen die Möglichkeiten der Messung weit über das einfache Zählen von Pixeln, z. B. von bestimmten Grauwerten, hinaus. Im Vorfeld der Bildanalyse am Binärbild werden häufig Methoden der Graubildverarbeitung eingesetzt, um eine sachgemäße Segmentierung zu ermöglichen.

\subsubsection{Graubildverarbeitung}

Die Graubildverarbeitung in einer Bildverarbeitungs- und Bildanalysesequenz dient der Bildverbesserung, um die Strukturen oder Objekte, die von Interesse sind, besser erkennen und segmentieren zu können. Die dabei verwendeten Pixeloperationen werden in linear und nichtlinear unterschieden. Bei linearen Pixeloperationen hängen die Grauwerte des Ausgangsbildes nur vom Eingangsbild ab. Beispiele sind Operationen wie Kontrastverstärkung durch Histogrammspreizung, Grauwertnormierung, Falschfarbencodierung, Ausleuchtungskorrektur und Kantenverstärkung. Bei nichtlinearen Pixeloperationen hängen die Grauwerte des Ausgangsbildes zusätzlich auch z.B. vom Ort, d.h. vom Bildinhalt $a b$, es handelt sich um Matrixoperationen. Hierzu gehören die verschiedenen Filteroperationen, wobei sich diese wieder in lineare (z.B. Hoch- und 
Tiefpassfilter) und nichtlineare (richtungsabhängige) Koeffizientenfilter unterscheiden lassen. Für alle genannten Operationen gilt, dass sie keinen wirklichen Informationsgewinn bieten, sondern bestimmte Strukturen oder Objekte stärker hervorheben.

\subsubsection{Binärbildgenerierung}

Die bereits angesprochene Segmentierung dient der Unterscheidung verschiedener Objekte und der Erstellung eines Binärbildes. Viele Messungen sind ausschließlich am Binärbild möglich. Per Konvention werden die Objekte, die von Interesse sind, mit weiß (Grauwert 255) kodiert, der Hintergrund mit schwarz oder dem Grauwert 0. Jeder Bildpunkt des Binärbilds besteht entweder aus der logischen Information 0 oder 1 . So wird wesentlich weniger Speicherplatz benötigt. Am Binärbild können logische Operationen (Boolsche Operatoren „und“, „oder“, „bedingtes oder“) und morphologische Operationen (s. u.) durchgeführt werden. Moderne Bildverarbeitungssysteme wie das KS400 bieten auch die Möglichkeit der Graubildmorphologie.

\subsubsection{Morphologische Operationen}

Die gebräuchlichsten morphologischen Operationen sind die Erosion, die Dilatation und die möglichen Kombinationen beider Methoden. Es handelt sich um die logische Verknüpfung einer Maske (die hier Strukturelement genannt wird) mit dem Ausgangsbild. Bei einem Binärbild wirkt sich dies auf die Ränder zwischen beiden Bereichen aus. Im Folgenden werden die morphologischen Operationen in Anlehnung an Serra (1982) und Eins \& Stiller (1995) erläutert:

Erosion: Wenn das Zentrum des Strukturelementes auf ein schwarzes Pixel fällt (logisch 0, Hintergrund), werden alle Pixel, die von der Maske bedeckt sind, auf 0 gesetzt. Das bewirkt eine Schrumpfung (Erosion) der weißen Objekte von außen nach innen.

- Dilatation: Wenn das Zentrum des Strukturelementes auf ein weißes Pixel fällt, (logisch 1, Objekt) werden alle Pixel, die von der Maske bedeckt sind, auf 1 gesetzt. Das bewirkt eine Ausdehnung (Dilatation) der Objekte.

Während die Erosion kleine Objekte verschwinden lässt und eine Trennung zerklüfteter Objekte bewirkt, werden solche Objekte bei der Dilatation verschmolzen und Löcher werden gefüllt. Eine Dilatation der Objekte entspricht der Erosion des Untergrundes und umgekehrt. Die morphologischen Operationen sind nicht umkehrbar, da Bildinformationen verloren gehen. Um die ungefähre Ausgangsgröße der Objekte beibehalten zu können, werden Kombinationen aus beiden Operationen eingesetzt:

Opening: Nach der Erosion wird eine Dilatation mit demselben Strukturelement durchgeführt. Dadurch werden verschmolzene Objekte zunächst getrennt und dann auf ihre ungefähre Ausgangsgröße zurückgeführt. Alle Objekte, die das Strukturelement in keinem Punkt vollständig enthalten, verschwinden.

Closing: Nach der Dilatation wird eine Erosion mit demselben Strukturelement durchgeführt. Das bewirkt die Verschmelzung fragmentischer und die Glättung zerklüfteter Objekte und eine Rückführung auf ihre ungefähre Ausgangsgröße. Löcher bzw. Hintergrund, die das Strukturelement in keinem Punkt vollständig enthalten, verschwinden. 
Die Wahl des Strukturelementes kann anwendungsbezogen erfolgen und so ein empfindliches Selektionskriterium darstellen. Mit den morphologischen Operationen sind Nachbarschafts-, Abstands-, Gestalts- und Größenanalysen möglich. Auf eine spezielle Anwendung des Opening-Verfahren wird in Kapitel 4.5 ausführlicher eingegangen.

\subsubsection{Objektmessung und stereologische Verfahren}

Gut ausgestattete Bildanalyseprogramme bieten die Möglichkeit, eine Vielzahl von Parametern an als solchen erkannten, d.h. segmentierten Objekten über eine Menüauswahl messen zu können. Dazu gehören als Primärparameter die Fläche, der Umfang bzw. die Länge von Linien, der Feret-(Tangenten) durchmesser und die Position in $\mathrm{x}$-, $\mathrm{y}$ Koordinaten sowie als Zählparameter die Anzahl. Davon können sekundäre Parameter wie der Durchmesser eines flächengleichen Kreises, der konvexe Umfang, Rundheit, Rauhigkeit, Formfaktor und Porosität bestimmt werden. Einige dieser Parameter erlauben den Einsatz der Stereologie. Dabei handelt sich um einen Ansatz, Tiefeninformationen, die durch die Auswertung von mikroskopischen Dünnschliffen verlorengegangen sind, durch statistische Aussage wieder $\mathrm{zu}$ gewinnen. Durch das Messen von zweidimensionalen Proben können Informationen über die dritte Dimension erlangt werden. So können Volumendichten, Oberflächendichten, Längendichten, und numerische Dichten statistisch kalkuliert werden.

\subsubsection{Erstellung von Anwendungsprogrammen für das KS400}

Die Bedienung des KS400 ist sowohl interaktiv über die Menüleiste als auch halbautomatisch mit lauffähigen Programmen möglich. Diese Makros können entweder interaktiv aufgezeichnet oder direkt in einem C-ähnlichen Code programmiert werden. Eine Kombination aus beiden Verfahren ist möglich, einige Befehle sind jedoch ausschließlich über die manuelle Eingabe verfügbar. Die Programme werden während der Ausführung interpretiert. So können umfangreiche und komplizierte Programmabfolgen einschließlich der Ausgabe von Messdaten festgehalten und routinemäßig angewendet werden. Für die vorliegende Arbeit wurden spezielle Programme zur Messung der fraktalen Dimension mit Boxcounting und Dilatation (Eins 1994) und zur Messung der Porengrößenverteilung mit dem Opening-Verfahren (Dathe et al. 1998) entwickelt. Diese Verfahren werden im folgenden Kapitel 4.5 genauer erläutert. Zusätzlich wurden weniger umfangreiche Makros z.B. für die Vorverarbeitung und Segmentierung der Eingangsbilder für die verschiedenen angewendeten mikroskopischen Verfahren erstellt. Zur Messung der Konnektivität wurde ebenfalls ein Makro programmiert. Auf die Bedeutung der Konnektivität für Transportprozesse im Boden wird in Kapitel 7 eingegangen.

\subsection{Anwendung der digitalen Bildverarbeitung}

Während die REM-Bilder bereits in einem für die Bildanalyse geeigneten Format vorlagen, mussten die LM-Bilder zuvor digitalisiert werden. Das war mit hoher geometrischer wie auch Farb- bzw. Grauauflösung durch Bearbeitung von Farbdias in einem hochauflösenden Diascanner (Nikon LS-20E ${ }^{\circledR}$ ) möglich. Einen Überblick über die Arbeitsschritte für die verschiedenen Messungen gibt Abb. 4.8. 


\section{Digitale Bildanalyse}

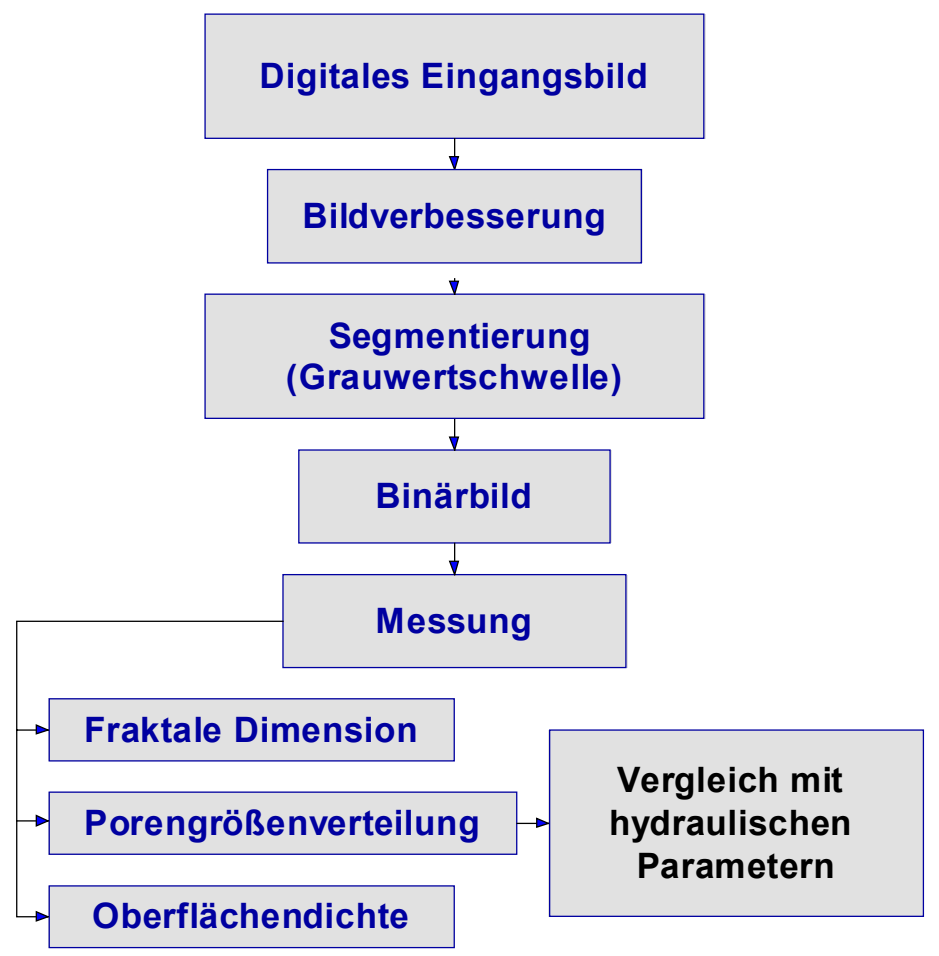

Abb. 4.8: Ablaufschema der digitalen Bildverarbeitung und Bildanalyse einschließlich der erhobenen Messgrößen und dem Vergleich mit hydraulischen Parametern.

\subsubsection{Bildvorbereitung}

Wie bereits erwähnt, wurde am Ausgangsbild so wenig wie möglich verändert, um den Untersuchungsgegenstand für die fraktale Dimension - die Grenzfläche zwischen Poren und Matrix - nicht zu verändern. Da einige der am Wild-Photomakroskop aufgenommenen Bilder nicht gleichmäßig ausgeleuchtet waren oder der Schliff unterschiedlich dick war, wurde vereinzelt eine sogenannte Shading-Korrektur vorgenommen. Hierfür wird das Originalbild mit einem Tiefpass von extremer Größe gefiltert. Dieses unscharfe Bild zeigt dann nur noch die Helligkeitsverhältnisse und wird vom Originalbild subtrahiert. Das Ergebnisbild ist von gleichmäßiger Helligkeit und kann so besser segmentiert werden.

Die geometrische Kalibrierung (d. h. die Einlesung des Maßstabs) erfolgte für die verwendeten mikroskopischen Verfahren in unterschiedlicher Weise. Für die analog an den Lichtmikroskopen aufgenommenen Bilder existieren Kalibriertabellen, die die Größe des realen Bildausschnitts in Abhängigkeit vom verwendeten Objektiv und Okular bzw. Tubusfaktor für das Kleinbildformat von 24 x $36 \mathrm{~mm}$ angeben. Dieser Faktor wurde als Kalibrierfaktor benutzt. Bei den REM Bildern wurde für jede Vergrößerungsstufe einer Bildserie der Maßstabsbalken mit dem entsprechenden Werkzeug für die geometrische Kalibrierung eingelesen und so die Pixelgröße skaliert. Die Werte sind in Tabelle 4.1 dokumentiert. In jedem Programmablauf wurde die aktuelle Skalierung angezeigt. 


\subsubsection{Segmentierung}

Für die eigentliche bildanalytische Messung mussten die Bilder segmentiert werden, d.h. das Ausgangsbild (Graubild der Tiefe 1 Byte beim REM, Farbbild der Tiefe 3 Byte für die Kanäle R, G, B bei der Lichtmikroskopie) wurde in ein Binärbild umgewandelt. Generell kann unterschieden werden in eine Segmentierung nach dem Grauwert und eine Segmentierung nach dem Farbkanal bzw. eine Kombination aus den drei Farbkanälen. In der vorliegenden Arbeit wurden auch die Farbbilder anhand der Grauwerte segmentiert. Hierfür können mathematische Algorithmen verwendet werden (z. B. Vogel \& Kretzschmar 1996) oder die Segmentierung erfolgt interaktiv, wenn eine klare bimodale Verteilung wie in Abb. 4.9 empirisch gemessen vorliegt. Das Programmpaket KS400 bietet hier die Möglichkeit, Ausgangs- und Binärbild $\mathrm{zu}$ vergrößern und die bei einer Veränderung der Grauwertschwelle (threshold) hinzukommenden oder wegfallenden Pixel farbig markiert darzustellen. Für die durchgeführten Arbeiten wurde nach dem interaktiven Verfahren gearbeitet und die Schwelle manuell gesetzt, so dass die Grauwerte der dunkelsten Poren am Porenmaximum des Histogramms gespiegelt werden (s. Abb. 4.9). Dabei wird eine Normalverteilung der zu den Poren gehörenden Häufigkeiten der Grauwerte angenommen.

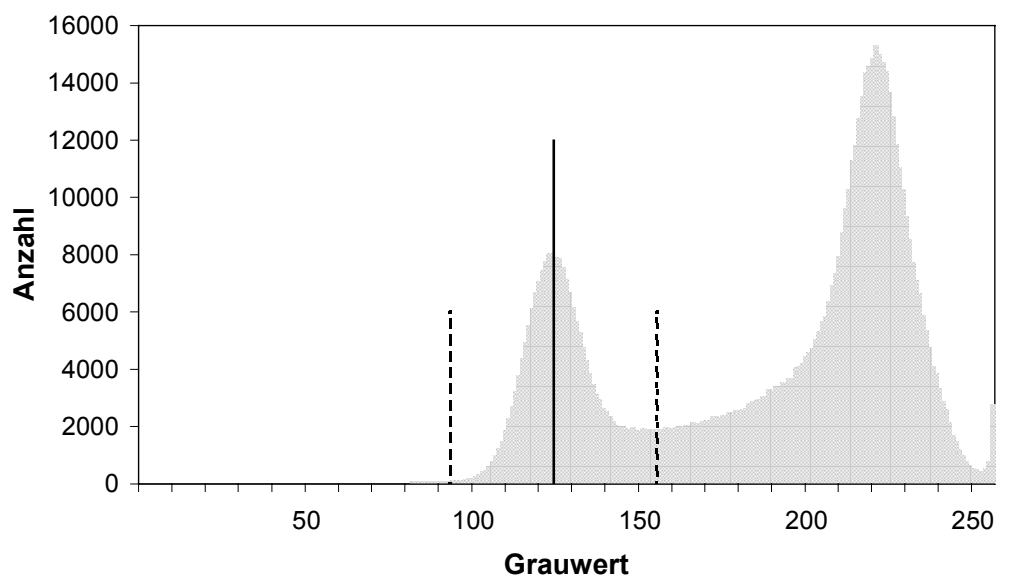

Abb. 4.9: Schematische Darstellung der Wahl der Segmentierungsschwelle, hier beim Grauwert 156 (rechte gestrichelte Linie). Der kleinste vorkommende Grauwert (94, linke gestrichelte Linie) wurde am Porenmaximum (durchgezogene Linie) gespiegelt.

\subsubsection{Bildnachbereitung}

Nur die 5000x vergrößerten REM Binärbilder bedurften einer Nachbereitung. Bei dieser Vergrößerungsstufe schimmerten Matrixanteile aus tiefer gelegenen Schichten durch das Gießharz hindurch, der Saum der einzelnen Körner erschien unscharf. Verursacht wurde dieser Effekt durch die im Vergleich zur Auflösung relativ große Eindringtiefe der Rückstreuelektronen. Hier wurde versucht, den Rand von Körnern scharf zu segmentieren, anschließend wurde „gescrappt“, d.h. scheinbare Poren von nur wenigen zusammenhängenden Pixeln wurden der Matrix zugeschlagen. Abschließend erfolgte bei jedem Binärbild der Vergleich mit dem Originalbild, um eine möglichst objektive Vorgehensweise zu gewährleisten. 


\subsection{Messung der fraktalen Dimension der Porenraumgrenzlinie}

Im Folgenden wird immer der Begriff „Porenraumgrenzlinie“ verwendet, da eine Linie in einem zweidimensionalen Bild gemessen wurde. Die Untersuchung der Porenraumgrenzfläche kann streng genommen nur im dreidimensionalen Raum erfolgen. Nach der von Mandelbrot et al. (1984) vorgestellten Methode der ,slit island technique“ ändert sich die fraktale Dimension (D) einer Struktur um genau den Wert 1, wenn von einer euklidschen (einbettenden) Dimension in die nächsthöhere (oder niedrigere) gewechselt wird. Dieser Ansatz wird von zahlreichen anderen Autoren angewendet (Tyler \& Wheatcraft 1989, Crawford et al. 1995, Pachepsky et al. 1996) und durch die Arbeiten von Moreau et al. (1999) bestätigt. Für die fraktale Dimension der Porenraumgrenzlinie $\left(D_{L}\right)$ bedeutet dieser Sachverhalt, dass durch die Addition von 1 das $D_{S}$ der Porenraumgrenzfläche (für den dreidimensionalen Raum) erhalten wird.

Nach der Segmentierung und der damit verbundenen Umwandlung des Ausgangsbildes in ein Binärbild wurden die Konturlinien durch die im KS400 implementierte graphische Funktion „Isohypsen“ klar erkannt (s. Abb. 4.10). Die bildanalytische Messung erfolgte direkt und ausschließlich an den Konturlinien einer Breite von einem Pixel zwischen Poren und Matrix. Für beide Verfahren, Boxcounting und Dilatation, wurde das Bild zunächst um einen Rand derart vergrößert, dass sich Boxen bzw. der Dilatationsschlauch ohne Randeffekte aufbauen konnten. Da jedes Bild als Rasteraufnahme des gesamten Dünnschliffs angesehen werden kann und die Konturlinien durch den Rand abgeschnitten werden, wurde dieser Rand lediglich an zwei Seiten hinzugefügt.

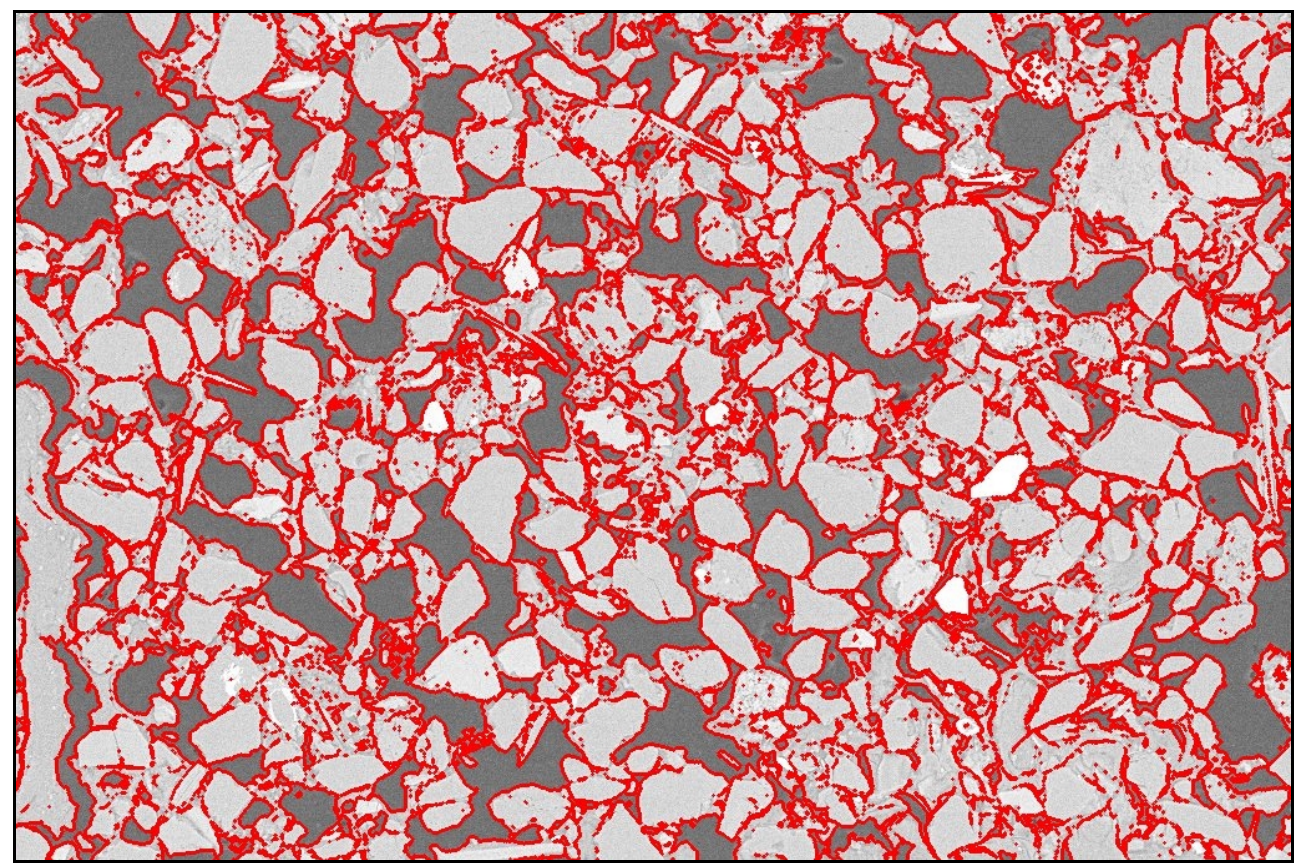

Abb. 4.10: Konturen der Grenzlinie Pore/Matrix über dem Originalbild. Die Abb. ist analog zu 4.2 e, und der besseren Deutlichkeit halber größer dargestellt. Die fraktale Dimension wurde ausschließlich an dieser Grenzlinie, die im Original nur eine Dicke von einem Pixel aufweist, gemessen. 


\subsubsection{Box Counting}

Das Boxcounting-Verfahren ist ein etabliertes Verfahren zur Messung der fraktalen Dimension. Es macht sich den in Kapitel 2 unter Gleichung 2.9 dargestellten Sachverhalt der Selbstähnlichkeit zu Nutze: Mit zunehmender Größe $\varepsilon$ der Boxen nimmt die Anzahl N der Boxen, die Teile des Objekts enthalten, ab. Bei fraktalen Objekten erfolgt die Abnahme proportional zur Boxgröße mit dem negativen Exponenten $D^{\mathrm{b}}$ (b steht für Boxcounting).

$$
\mathrm{N}(\varepsilon) \propto \varepsilon^{-\mathrm{D}^{\mathrm{b}}}
$$

Im verwendeten Programm wurde das gesamte Bild in einer Messschleife mit quadratischen Boxen wachsender Seitenlänge bedeckt. Anfangsgröße und Schrittweite konnten interaktiv eingegeben werden. Für die durchgeführten Messserien wurde immer mit einer Anfangsgröße von einem Pixel und einer Schrittweite von eins, die zu jeweils ungeraden Pixelseitenlängen der Boxen führt, gearbeitet.

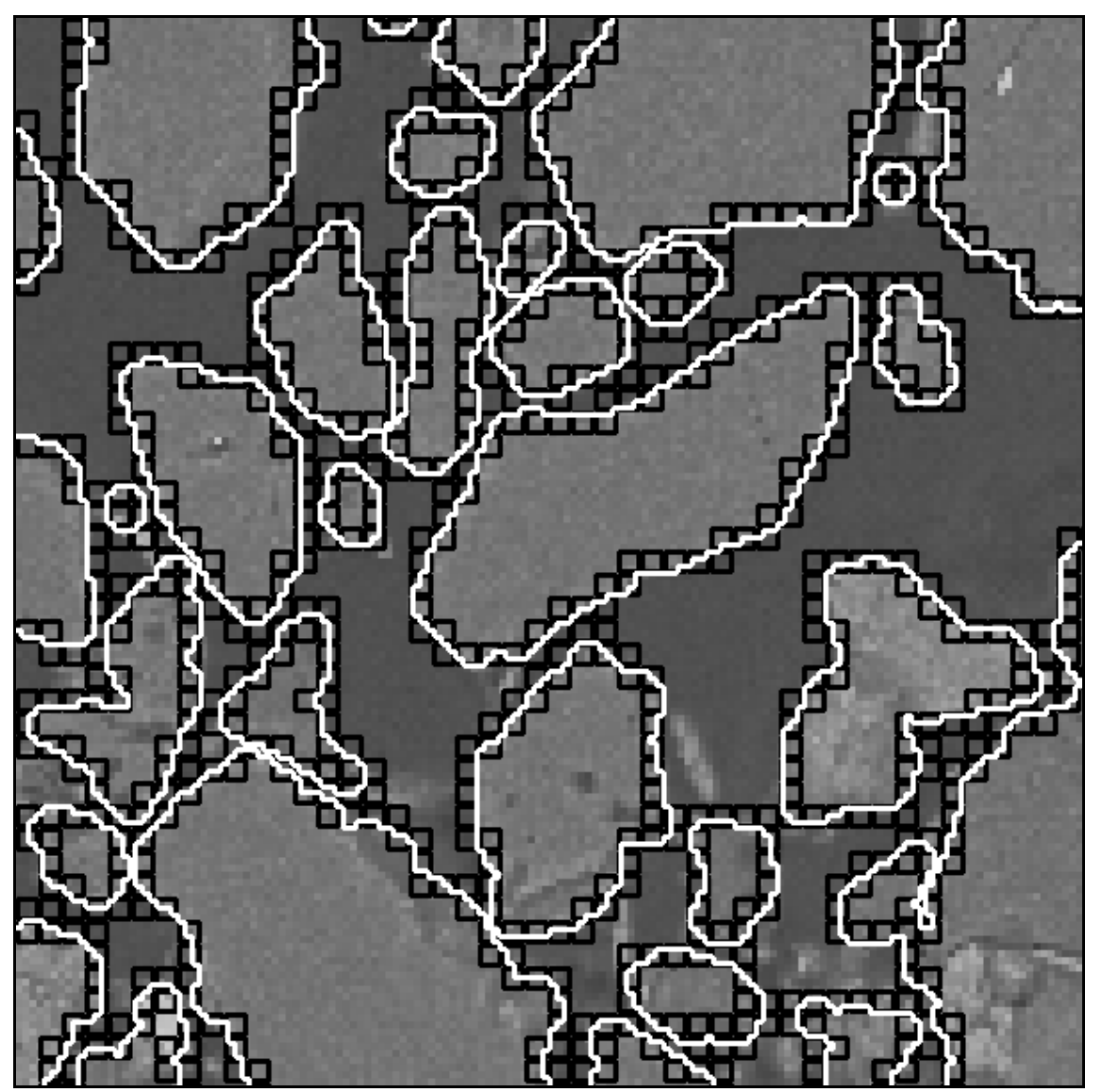

Abb. 4.11: Beispiel (Momentaufnahme) für das Boxcounting-Verfahren an einem Bildausschnitt. Die ausgewählte Skalierung wird durch die Boxgröße angezeigt. Die Messung findet ausschließlich an der hier weiß dargestellten Konturlinie statt, die Poren erscheinen dunkel und die Matrix hell.

In jedem Messschritt wurden die Seitenlänge der verwendeten Boxgröße und die Anzahl der Boxen, die ein oder mehrere Konturpixel beinhalten, in einer Datei abgespeichert. Die Werte wurden sowohl absolut als auch dekadisch eingelesen. Die Boxgröße wurde nach erfolgter Eichung in $\mu \mathrm{m}$ eingelesen und ausgegeben. Pixel der Konturlinie, die sich auf dem rechten oder unteren Rand der Boxen befanden, wurden nicht berücksichtigt, sondern 
den anschließenden Boxen am oberen oder linken Rand zugeschlagen, so dass keine Pixel durch das Raster verdeckt waren (s. Abb. 4.11). Das Programm lieferte neben der Tabelle der Messwerte einen log-log-Plot, in dem die Anzahl (N) der Boxen, die mindestens ein Pixel der Konturlinie beinhalten, über der Boxgröße ( $\varepsilon$ ) aufgetragen sind. D kann dann aus der Steigung aller Daten abgelesen werden. Für die weitere Auswertung wurden die Daten in das Tabellenkalkulationsprogramm Excel kopiert. Für Boxcounting erfolgte die Messung so lange, bis für die Anzahl Boxen, die die Konturlinie beinhalten, dreimal in Folge der gleiche Wert auftauchte.

\subsubsection{Dilatation}

Für Dilatation wurde die Konturlinie dilatiert, wobei im KS400 verschiedene Strukturelemente zur Verfügung stehen. Um Anisotropieeffekte zu vermeiden, wurde nach Möglichkeit ein Kreis verwendet. Auch hier konnte die Anfangsgröße und Schrittweite des strukturierenden Elements interaktiv eingegeben werden. Für den Startwert von einem Pixel ergab der Meßwert die Gesamtlänge der Konturlinie. Mit zunehmender Dicke des die Konturlinie umhüllenden Dilatationsschlauches nimmt die Gesamtlänge $a b$, da kleine Details geglättet werden (s. Abb. 4.12).

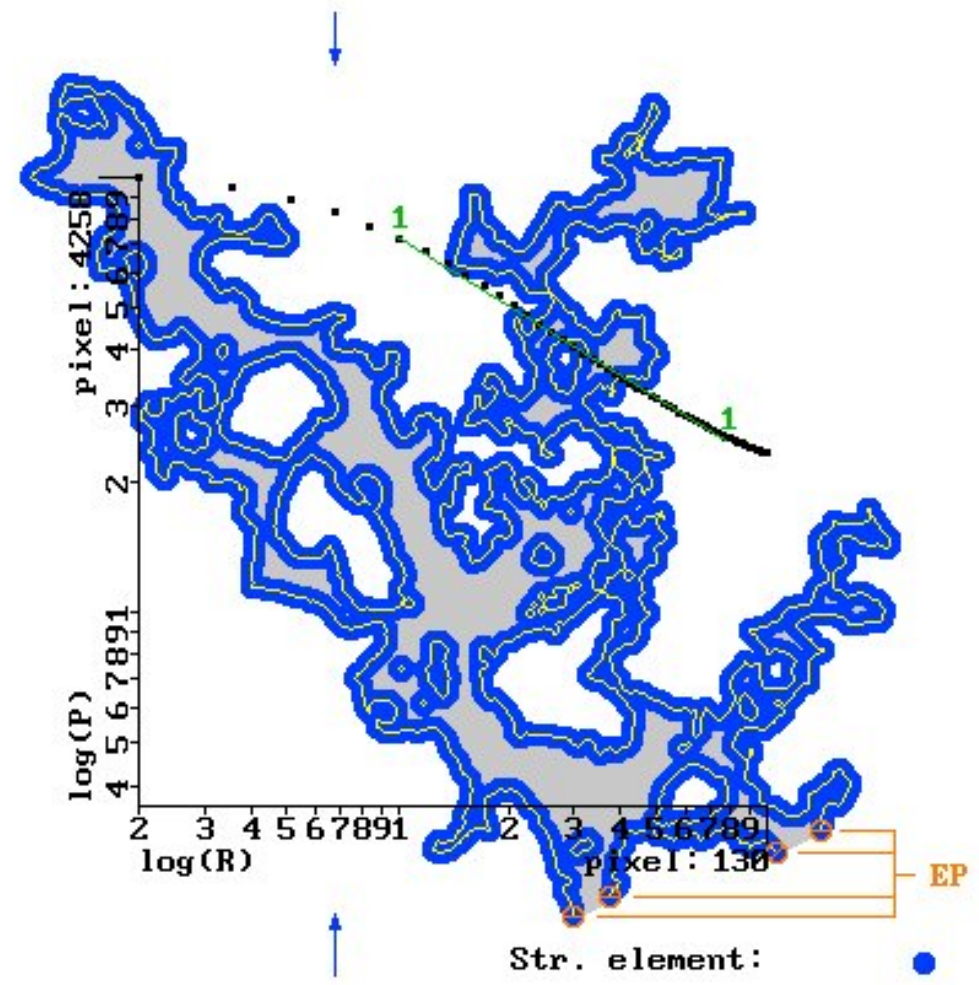

Abb. 4.12: Dilatation am Beispiel des vernetzten Porensystems im Boden (grau und blau innerhalb der gelben Kontur), wie es an polierten Oberflächen von eingebetteten Bodenproben beobachtet werden kann. Die ausgewählte Skalierung wird durch die Dicke der Umhüllenden (blau), sowie durch das hier verwendete oktagonale strukturierende Element und die Markierungspfeile auf der Skalenachse angezeigt. 1 - 1 markiert die angepasste Gerade zwischen unterem (LCO) und oberem Cutoff (UCO), D ist die aus der Steigung berechnete fraktale Dimension. Die Dilatation von Konturendpunkten (EP) führt zur Überschätzung der Fläche und nach Gl. 4.2 zur Unterschätzung von D. Dieser Faktor kann korrigiert werden (Eins, 1995). 
Die Berechnung der Gesamtlänge erfolgte unter Berücksichtigung der Richtungsänderungen. Dafür wurden alle Abweichungen der Konturlinie von einer Geraden durch Richtungsfilter erkannt, die jeden im Pixelraum möglichen Winkel annehmen und zählen. Die Summen der verschiedenen möglichen Richtungsänderungen gingen als Anisotropieindizes in die Berechnung der Länge im ersten Messschritt ein. Bei der Verwendung eines Kreises als strukturierendem Element wurde die Länge in den folgenden Schritten als Quotient aus Fläche und Durchmesser berechnet. Die fraktale Dimension $\mathrm{D}^{\mathrm{d}}$ (d steht für Dilatation) ergibt sich analog zu Gleichung 2.8 und kann wiederum aus der Steigung eines log-log Plots, in dem die Länge der Umhüllenden L über ihrem Durchmesser $\varepsilon$ aufgetragen wird, abgelesen werden.

$$
\mathrm{L}(\varepsilon) \propto \varepsilon^{1-D^{\mathrm{d}}}
$$

Wie beim Programm zur Messung der fraktalen Dimension mit Boxcounting wurden am Ende jedes Messschritts die absoluten und die logarithmierten Werte für die Dicke des Dilatationsschlauches und die damit gemessene Länge in einer Datei gespeichert (s. Abb. 4.12, in der ein Messschritt und der gesamte log-log-Plot gleichzeitig dargestellt sind). Der log-log-Plot wurde für alle Messwerte angezeigt und die Daten zur Weiterverarbeitung in EXCEL gespeichert. Bei der Dilatation wurde als obere Abbruchbedingung eine Schlauchdicke von $1 / 3$ des in dem entsprechenden Bild gemessenen maximalen Feretdurchmessers definiert.

\subsubsection{Untersuchung der Auswirkung verschiedener Auflösungen}

Das Rasterelektronenmikroskop Leo Gemini bietet die Möglichkeit, denselben Bildausschnitt mit verschiedenen Auflösungen abzurastern und den Bildinhalt in vier verschiedenen Auflösungsstufen abzuspeichern. Um die Auswirkung der Auflösung auf das Messergebnis in Hinblick auf die fraktale Dimension zu untersuchen, wurden von einem Dünnschliff Bilder von drei Vergrößerungsstufen (150x, 500x und 3000x) in den folgenden vier Auflösungen erstellt:

$$
\begin{aligned}
& 512 \times 384 \text { Pixel } \\
& 1024 \text { x } 768 \text { Pixel } \\
& 2048 \times 1536 \text { Pixel } \\
& 3072 \text { x } 2304 \text { Pixel }
\end{aligned}
$$

An den erhaltenen Bildern wurde mit den oben beschriebenen Methoden die fraktale Dimension gemessen und die Ergebnisse verglichen (s. Tab. 6.11, 6.12 und Abb. 6.13).

\subsubsection{Messung der Porengrößenverteilung}

Die Porengrößenverteilung wurde mit dem in Abschnitt 4.4 .4 beschriebenen und zur Porenklassifizierung von Vogel (1996) benutzten Opening-Verfahren ermittelt. Die Messung erfolgte am Binärbild. In dem für das KS400 erstellten Programm wurde mit einem auswählbaren Strukturelement zunächst eine Erosion durchgeführt und dann mit dem gleichen Strukturelement dilatiert (s. Abb. 4.13). Um Anisotropieeffekte zu vermeiden, wurde wie bei der Dilatation nach Möglichkeit ein Kreis verwendet. In der Software traten bei extrem komplexen Bildern bei der konturbasierten Kreisapproximation 
interne Speicherprobleme auf und es musste auf das pixelbasierte Achteck ausgewichen werden. In einer Messschleife wurde der Durchmesser des Strukturelementes kontinuierlich vergrößert. Auch hier konnten Anfangsdurchmesser und Schrittweite frei gewählt werden. Vor dem ersten Opening-Schritt wurde die Bildfläche und die gesamte, als Poren segmentierte Fläche gemessen. Nach jedem Opening-Schritt wurde der Durchmesser des Strukturelements, die in diesem Schritt verschwundene Porenfläche, die Anzahl der verschwundenen Elemente sowie die Anzahl der verbleibenden Poren gemessen. Durch die Zuordnung der wegfallenden Porenfläche zum Durchmesser des in diesem Messschritt verwendeten Strukturelements konnte der Flächenanteil einer bestimmten Porenklasse bestimmt werden. Die Wiederholung mit größer werdenden Strukturelementen führte zu einer Klassifizierung der Porengrößenverteilung.
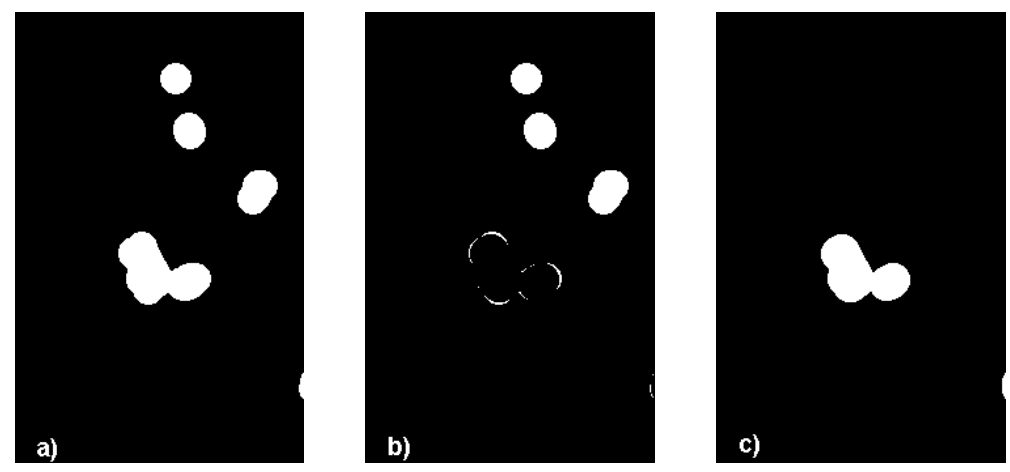

Abb. 4.13: Opening-Verfahren; a) binäres Ausgangsbild; b) Objekte die kleiner als das kreisförmige Strukturelement sind und durch die Erosion aus dem Bild verschwinden; c) Ergebnisbild nach der Dilation mit den Objekten, die größer als das in diesem Schritt verwendete Strukturelement sind.

Das Ergebnis wurde als Häufigkeit der Flächenanteile von Poren bestimmter Durchmesserklassen dargestellt (s. Kap. 5.3, Abb. 5.10). Um einen Vergleich mit den entsprechenden hydraulischen Prozeßparametern zu ermöglichen, wurden die Durchmesser des Strukturelementes in den Betrag der Wasserspannung umgerechnet, bei der die Poren nach der Kapillarengleichung entwässern würden (s. Gl. 2.21).

\subsection{Bodenphysikalische Meßmethoden}

Ziel dieser Untersuchungen war die Charakterisierung der bezüglich fraktaler Dimensionen bildanalytisch untersuchten Bodenproben anhand von klassischen bodenphysikalischen Methoden. Eine ausführliche Darstellung, insbesondere von Messungen an Einzelaggregaten, findet sich bei Friedrichs (1999). Es wurden die Phasenanteile bestimmt und Wasserretentionen und Wasserleitfähigkeiten an Stechzylinderproben gemessen.

Zusätzlich zu den experimentell ermittelten Retentionskurven und Wasserleitfähigkeitsfunktionen wurden die Anpassungsparameter $\left(\mathrm{a}, \mathrm{n}, \theta_{\mathrm{r}}, \theta_{\mathrm{s}}\right)$ für das van Genuchten-MualemModell (van Genuchten 1980) zur mathematischen Beschreibung der Retentionskurve bestimmt und aus den Parametern die ungesättigte Wasserleitfähigkeit berechnet (s. Gl. 2.29 und 2.30 in Kapitel 2.3). Dies erfolgte sowohl unter der Annahme einer uni- als auch einer bimodalen Porenverteilung (gekoppelte van Genuchten-Gleichung, vgl. Durner 1991, Diekkrüger 1992). 


\subsubsection{Bestimmung der Phasenanteile}

Die Korngrößenanalyse wurde mit der kombinierten Sieb- und Pipetiermethode (verändert nach Schlichting et al., 1995) durchgeführt. Die 100 und $250 \mathrm{~cm}^{3}$ Stechzylinderproben wurden nach Ofentrocknung bei $105{ }^{\circ} \mathrm{C}$ gewogen und die Lagerungsdichte berechnet. Die Dichte der Festsubstanz wurde durch die Einwaage von lufttrockenem, feingemörserten Boden in ein definiertes Volumen von Xylol und anschließende Wägung bestimmt (Schlichting et al. 1995). Aus der Dichte der Festsubstanz $d_{F}$ und der Lagerungsdichte $d_{B}$ wurde nach Gl. 2.19 das Gesamtporenvolumen $V_{P}$ bestimmt.

\subsubsection{Ermittlung von Retentionskurven}

Die Retentionskurven wurden für die Tiefen 0-5 cm, 20-25 cm und 70-75 cm des untersuchten Bodenprofils (s. Tab. 3.2) an $100 \mathrm{~cm}^{3}$ Stechzylinderproben mit je 10 Parallelen nach der Desorptionsmethode ermittelt. Bis zu einem Unterdruck von $100 \mathrm{hPa}$ erfolgte die Entwässerung auf dem Sandbett einer Eijkelkamp-Unterdruckapparatur (Giesbeek, Niederlande), oberhalb von $100 \mathrm{hPa}$ wurde nach der Überdruckmethode mit keramischen Platten in Drucktöpfen der Firma Soil Moisture (Santa Barbara, Kalifornien) gearbeitet. Für die Untersuchung bei pF 4,2 wurden Stechringe mit einem Volumen von ca. $6 \mathrm{~cm}^{3}$ anfertigen. Die Einzelaggregate wurden zur besseren Handhabung mit Paraffin in einem flachen Plastikzylinder fixiert und ihre Unterseite sauber abgeschnitten, um den Kontakt zum Sandbett bzw. zur porösen Platte zu gewährleisten.

\subsubsection{Messung der gesättigten Wasserleitfähigkeit}

Die Bestimmung der gesättigten Wasserleitfähigkeit, erfolgte ebenfalls an $100 \mathrm{~cm}^{3}$ Stechzylinderproben an einem Permeameter der Firma Eijkelkamp (Giesbeek, Niederlande). Für stark durchlässige Proben wurde ein konstanter, für gering durchlässige Proben ein abnehmender hydraulischer Gradient verwendet. Die Messung der gesättigten Wasserleitfähigkeit an Einzelaggregaten erfolgte nach der bei Gunzelmann (1990) und Junkersfeld (1995) beschriebenen, modifizierten Methode eines Haubenpermeameters nach Hartge (1966).

\subsubsection{Messung der ungesättigten Wasserleitfähigkeit}

Die Beschreibung der Bestimmung der ungesättigten Wasserleitfähigkeit soll hier etwas ausführlicher erfolgen. Übersichten über verschiedene Messverfahren im Labor und im Gelände einschließlich mathematischer Methoden zur Auswertung bieten z. B. Opara-Nadi (1979), Plagge (1991) und Stolte et al. (1994). Es wurde eine Ku-Apparatur der Firma UGT (Müncheberg) benutzt. Das Gerät (s. Abb. 4.14) ermittelte die ungesättigte Wasserleitfähigkeit unter quasistationären Fließbedingungen an $250 \mathrm{~cm}^{3}$ Stechzylinderproben und konnte mit bis zu 10 Proben in einem Messdurchgang bestückt werden. Das Prinzip dieser Labormethode besteht darin, Wasser aus der zuvor aufgesättigten Probe verdunsten $\mathrm{zu}$ lassen und in zeitlichen Intervallen den Gewichtsverlust der Probe sowie mit Tensiometern den Gradienten der Wasserspannung zu bestimmen (s. u. a. Becher (1971), Schindler (1980) und Krahmer (1987)). 


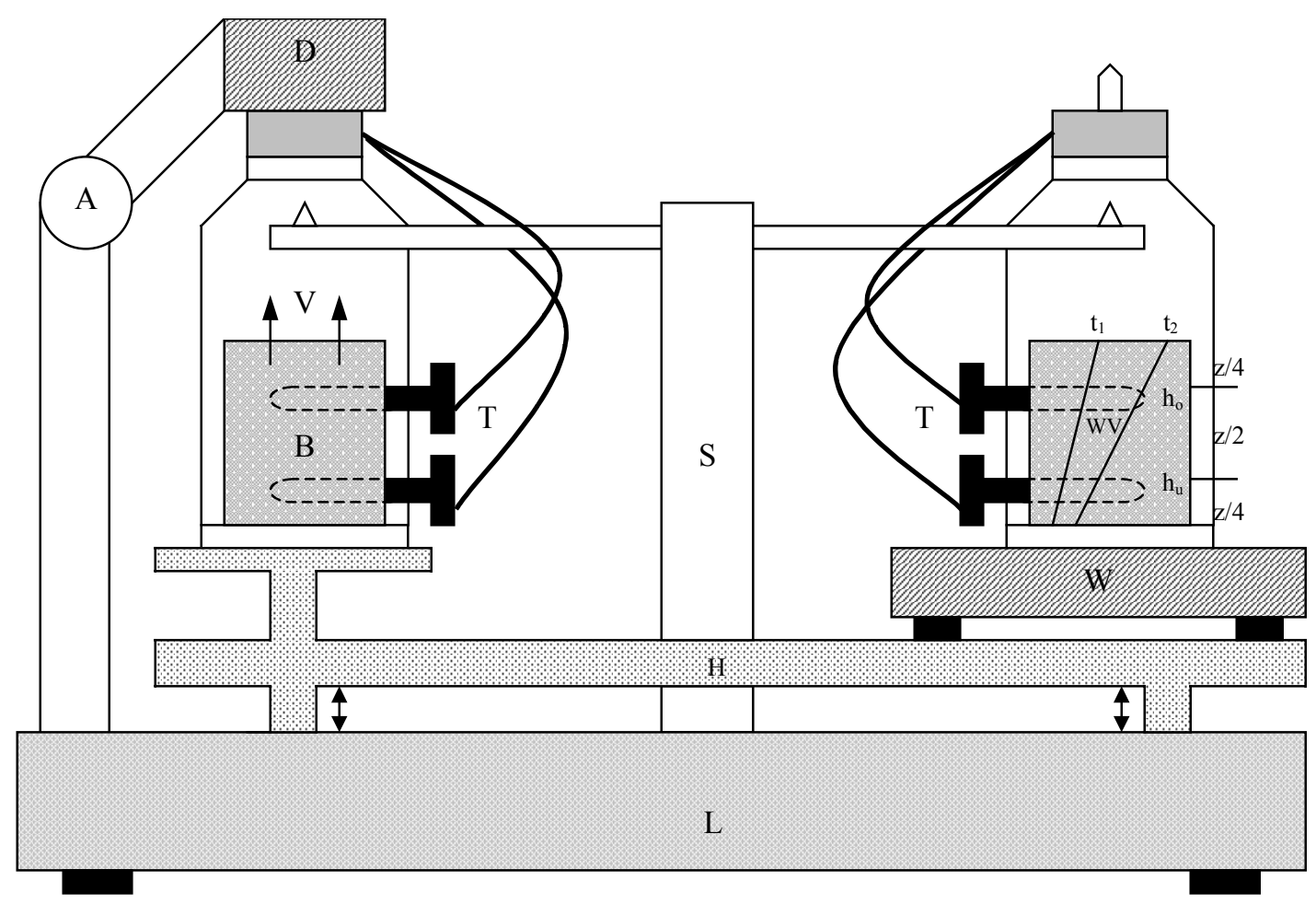

Abb. 4.14: Schematischer Aufbau der Ku-Apparatur zur Messung der ungesättigten Wasserleitfähigkeit. A: Tensionsaufnahmearm, B: Bodenprobe, D: Tensionsaufnehmer, H: Hubtisch, L: Logger- und Antriebseinheit, S: Schwenkarm, T: Tensiometer, V: Verdunstung, W: Waage, WV: Wasservolumen, z: Stechzylinderhöhe, t: Zeit, h: Einbauhöhen der Tensiometer (aus Friedrichs, 1999).

Die Stechzylinder befanden sich in Halterungen und waren in einem kreisförmigen, beweglichen Karussell angeordnet. Im zeitlichen Abstand von ein bis sechs Minuten wurde jede Probe unter den Tensiometeraufnahmearm gehoben, der die Abfrage der Wasserspannungsdaten ermöglichte, und auf die Waage gesetzt. So konnte mit nur einer Waage und einer Datenerfassungskarte gearbeitet werden. Mit einem PC wurde die Anlage gesteuert und die Daten ausgelesen. Vor Versuchsbeginn wurden die Proben aufgesättigt und in den Halterungen befestigt, die basale Abdichtungen aufwiesen. Anschließend wurden je zwei Tensiometer in drei $\mathrm{cm}$ Abstand in vorgebohrte, horizontale Löcher installiert. $\mathrm{Zu}$ Versuchsbeginn waren die Stechzylinder mit Deckeln abgedeckt, um einen Wasserverlust durch frühzeitig einsetzende Verdunstung $\mathrm{zu}$ vermeiden. Die Datenerfassung wurde gestartet und die Deckel entfernt, wenn sich zwischen den Tensiometern ein hydraulisches Gleichgewicht ausgebildet hatte. Dann setzte die Verdunstung ein. Durch die gleichzeitige Messung der Wasserspannung in zwei Tiefen und der Gesamtmasse von Probe plus Befestigungsvorrichtung konnte durch Rückwägung die Verdunstung pro Zeit bestimmt werden. Mit Hilfe der Tensiometerdaten ließ sich bei definiertem Querschnitt der Wasserfluss durch die Probe angeben. Die ungesättigte Leitfähigkeit K wurde dann von der zur Anlage gehörenden Software nach folgendem Verfahren berechnet:

Über einen bestimmten Zeitabschnitt $\Delta \mathrm{t}$ wird der hydraulische Gradient $\Delta(\psi-\mathrm{z}) / \Delta \mathrm{z}$ als annähernd konstant angesehen. Nach der Darcy-Gleichung ergibt sich der Volumenstrom pro Querschnittsfläche (Q) als 


$$
\mathrm{Q}=\mathrm{K} \cdot \frac{\Delta(\psi-\mathrm{z})}{\Delta \mathrm{z}}
$$

$$
\begin{aligned}
& \text { mit } \Delta \psi=\psi_{\text {oberes Tensiometer }}-\psi_{\text {unteres Tensiometer }} \\
& \Delta \mathrm{z}=\mathrm{z}_{\text {oberes Tensiometer }}-\mathrm{z}_{\mathrm{unteres}} \text { Tensiometer }
\end{aligned}
$$

Für die Versuchsanordnung kann der Volumenstrom auch als Quotient des verdunsteten Wasservolumens q pro Querschnittsfläche A und Zeitintervall $\Delta \mathrm{t}$ errechnet werden:

$$
\mathrm{Q}=\frac{\Delta \mathrm{q}}{2 \mathrm{~A} \cdot \Delta \mathrm{t}}
$$

Durch Einsetzen von Gl. 4.4 in Gl. 4.3 und Umstellen nach k ergibt sich für den Probenabschnitt zwischen den Tensiometern (Schindler 1980):

$$
\mathrm{K}=\frac{\Delta \mathrm{q}}{2 \cdot \mathrm{A} \cdot \Delta \mathrm{t}} \cdot \frac{\Delta \mathrm{z}}{\left(\psi_{\mathrm{o}}-\psi_{\mathrm{u}}\right)-\Delta \mathrm{z}}
$$

Ferner ist es möglich, aus den aus oberem und unterem Tensiometer gemittelten Daten und den jeweils zugehörigen Wassergehalten eine pF-Kurve innerhalb des Messbereiches der Tensiometer (bis max. $850 \mathrm{hPa}$ ) zu erstellen.

Somit wurden alle Messgrößen erhoben, die zur Modellierung des Wassertransports im Boden (Gl. 2.24) benötigt werden und es liegen zusätzliche Informationen über die Textur des untersuchten Bodens vor. 


\section{$5 \quad$ Mathematische Auswertung und Statistik}

Für jedes Einzelbild lagen drei Dateien (je eine für Boxcounting, Dilatation und die Messung der Porengrößenverteilung) mit den bildanalytisch gewonnenen Rohdaten im Excel Format vor. Es wurden im Wesentlichen die REM-Aufnahmen für drei Dünnschliffe ausgewertet, die im Folgenden die Namen Leo7, Leo8 und Leo9 tragen. Die Porengrößenverteilung wurde für Leo7 und Leo8 untersucht. Die mit dem WildPhotomakroskop erstellten lichtmikroskopischen Bilder (s. Kapitel 4.3.1) wurden von horizontalen und vertikalen Schliffen genommen und an ihnen die fraktalen Dimensionen und die Porengrößen-verteilungen ermittelt. Diese Serien tragen die Namen WK2hor und WK2ver.

Für die Bestimmung der fraktalen Dimension wurde mit der Menüfunktion „Trendlinie“ für die logarithmiert eingelesenen Daten eine lineare Regression nach der Methode der kleinsten Abweichungsquadrate (least squares) berechnet und sowohl als Grafik als auch als Geradengleichung angezeigt. Als Bestimmtheitsmaß wurde der quadrierte Produktmomenten-Korrelationskoeffizient angegeben. Für jede Vergrößerungsstufe der REM-Aufnahmen und jeden Schliff bzw. jede Wild-Serie wurde ein einheitlicher Datenbereich festgelegt und mit zwei verschiedenen Methoden die Mittelwerte gebildet (s. Abb. 5.1 und Kap. 5.2).

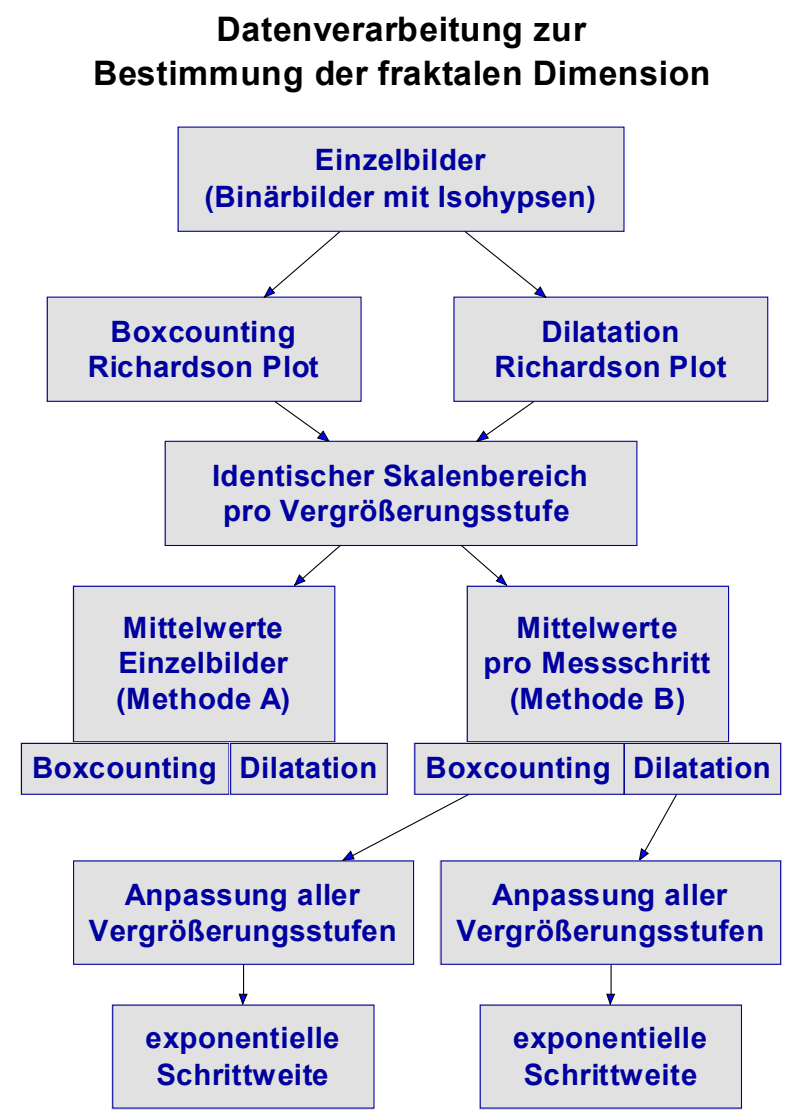

Abb. 5.1: Methoden der Datenverarbeitung zur Bestimmung der fraktalen Dimension aus den mit Boxcounting und Dilatation gewonnenen Daten. Zunächst wurden alle Bilder einzeln ausgewertet und anschließend ein einheitlicher Skalenbereich für jede Vergrößerungsstufe festgelegt. Die Mittelwerte für alle Vergrößerungsstufen wurden addiert und für jeden Schliff mit einem robusten Schätzverfahren eine bzw. zwei Geraden angepasst. Hierfür wurden lineare und exponentielle Schrittweiten verwendet. 
Später wurden die mit dem Boxcounting- bzw. dem Dilatations-Verfahren gemessenen Rohdaten für jede Methode und Vergrößerungsstufe gemittelt. Anschließend wurden die Achsenabschnitte addiert, um eine Gerade oder zwei Geraden für den gesamten Datenbereich anzupassen zu können. Diese Anpassung erfolgte sowohl für linear als auch für exponentiell verteilte Skalenschritte mit einem in $\mathrm{C}^{++}$programmierten robusten Schätzverfahren, das nach der Methode der lokalen M-Schätzer mit einer Optimierung mittels Minimierung der absoluten Abweichungen arbeitet (Press et al. 1992). Da die Residuen über den gesamten Datenbereich eine systematische Abweichung von einer Geraden aufwiesen, wurde abschließend eine asymptotisch verlaufende Funktion angepasst, die die Abhängigkeit der fraktalen Dimension von der Auflösung bzw. der Größe des skalierenden Elementes beschreibt.

Bei der Messung der Porengrößenverteilung wurden die Rohdaten pro Vergrößerungsstufe und Schliff ebenfalls gemittelt und dann in Richtung großer Poren als Summenkurve aufgetragen. So konnten die Ergebnisse direkt mit der Retentionskurve verglichen werden. Die Verteilung der Porenflächenhäufigkeiten über den gesamten Datenbereich wurde durch Normierung der Klassenbreiten ermittelt.

\subsection{Berechnung der fraktalen Dimension durch lineare Regression}

Die Berechnung der fraktalen Dimension D erfolgte analog zu den logarithmierten Gleichungen 4.1 und 4.2. In einem doppellogarithmischen Plot kann die fraktale Dimension aus der Steigung abgeleitet werden. Man erhält

$$
\log N(\varepsilon)=-D_{L}^{b} \log \varepsilon+c
$$

für das Boxcounting-Verfahren bzw.

$$
\log \mathrm{L}(\varepsilon)=\left(1-\mathrm{D}_{\mathrm{L}}^{\mathrm{b}}\right) \log \varepsilon+\mathrm{c}
$$

für das Dilatations-Verfahren.

Der y-Achsenabschnitt c stellt die logarithmierte Anzahl der Boxen dar, die bei der Größe des skalierenden Elementes von $1 \mu \mathrm{m}$ die Konturlinie beinhalten würden. Der Summand 1 in Gleichung 5.2 erklärt sich aus den unterschiedlichen Dimensionen der verwendeten „Messsonde“: Das Zählen von Kästchen (Boxcounting) entspricht der Messung mit einer Sonde der euklidischen Dimension 0, einer Anzahl (s. auch Gl. 2.9). Bei der Dilatation wird die Länge der Umhüllenden bestimmt, was der Messung mit einer Länge der euklidischen Dimension 1 entspricht (s. auch Gl. 2.8).

\subsubsection{Bestimmung des verwendeten Datenbereichs (Cutoffs)}

Werden für die lineare Regression alle Wertepaare im log-log Plot herangezogen, zeigte sich für Strukturelemente einer Größe von wenigen Pixeln eine methodenbedingte Abweichung von einer Geraden. Dieses Phänomen ist in der Literatur bekannt (z. B. Rigaut et al., 1983; Banerji, 1987; Cross, 1994) und wird u. a. auf die sogenannte Pixelrauhigkeit zurückgeführt. Sie bezeichnet den Einfluss der Form der einzelnen Pixel, 
die wiederum die Rasterung des Bildes beschreibt. Hinzu kommt die Tatsache, dass der Grauwert eines Pixels das Integral der Grauwerte über die Fläche von der Größe dieses Pixels auf dem Präparat darstellt. Im Bereich weniger Pixel wird so die Messung systematisch in Richtung kleinerer D verfälscht (s. Abb. 5.2 a), weshalb beispielsweise die ersten fünf Wertepaare nicht berücksichtigt werden (unterer Cutoff). In der vorliegenden Anwendung zeigte sich, dass ein einziges Wertepaar (für die Größe des Strukturelementes von wenigen Pixeln) einen relevanten Einfluss im Bereich der ersten Nachkommastelle auf den Wert für D hat. Dies gilt für Boxcounting und für Dilatation.
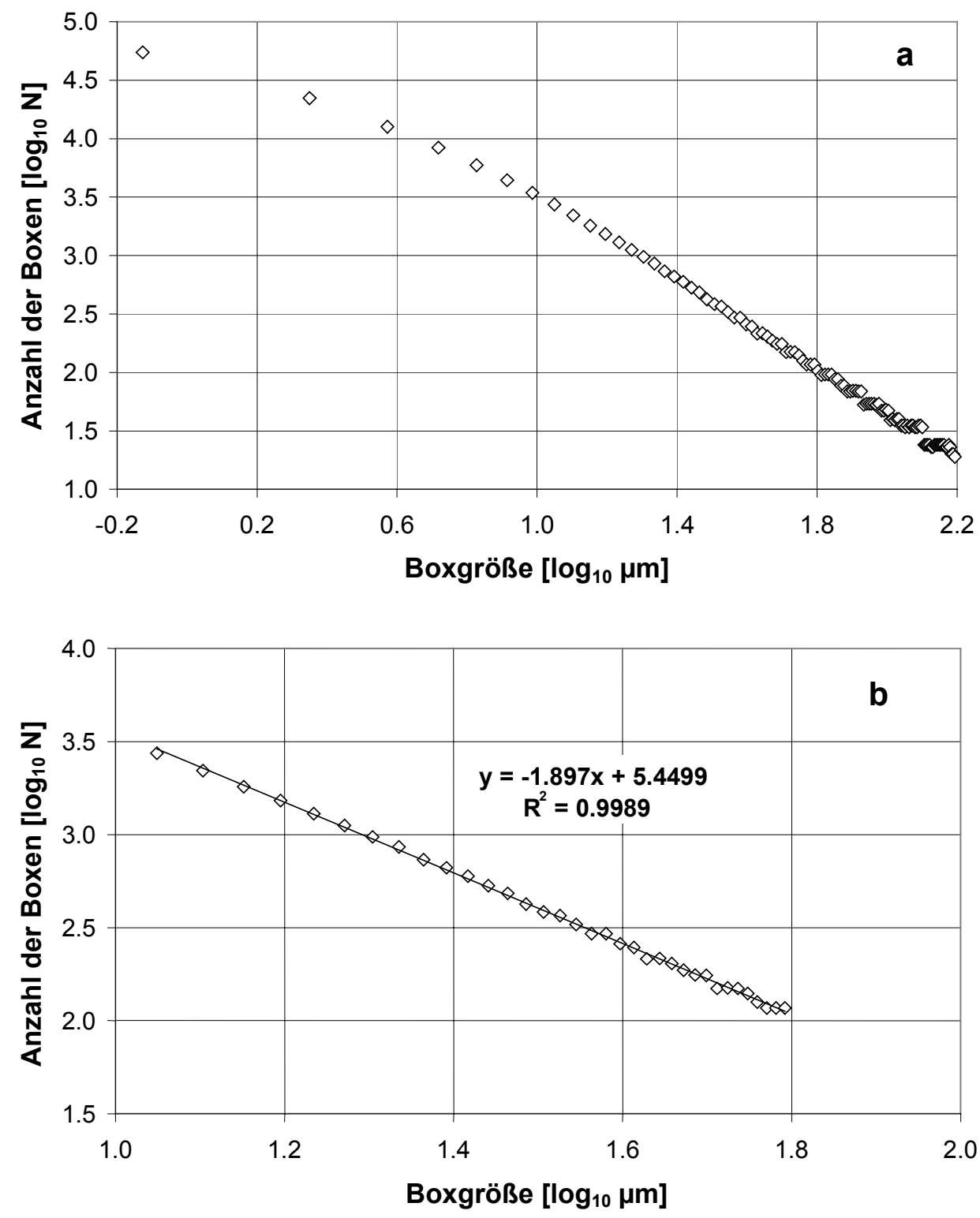

Abb. 5.2: Richardson Plots für die in Abb. 4.2 d und e dargestellte REM-Aufnahme. (a) Alle Messwerte mit Boxen einer Seitenlänge ab einem Pixel (bei einer Auflösung von $0,75 \mu \mathrm{m} / \mathrm{Pixel}$ ) bis zum Abbruch des Makros (bei 155,97 $\mu \mathrm{m}$ ) des Boxcounting-Verfahrens sind dargestellt. (b) Der als linear ausgewählte Bereich zwischen dem unteren $(11,19 \mu \mathrm{m})$ und dem oberen $(61,94 \mu \mathrm{m})$ Cutoff aus (a) wurde nach beschriebenen Methode bestimmt. 


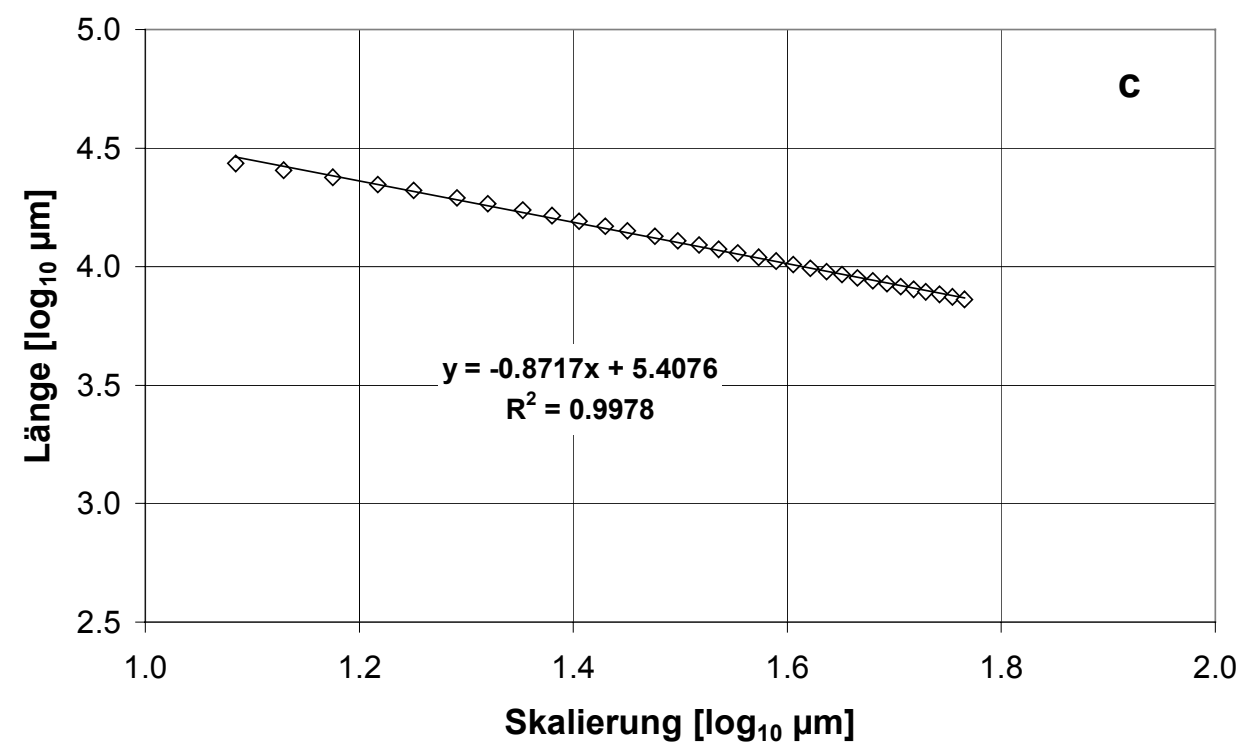

Abb. 5.2: Richardson Plots für die in Abb. 4.2 d und e dargestellten REM Aufnahme. (c) Entsprechender Richardson Plot für das Dilatations-Verfahren.

Die obere Abbruchbedingung (oberer Cutoff) trägt dem Sachverhalt Rechnung, dass ab einem bestimmten Verhältnis zwischen der Größe der untersuchten Objekte und der Größe des verwendeten Strukturelementes eine Messung nicht mehr sinnvoll ist. Das weitere Vergrößern der Messsonde, also der Boxgröße oder des Durchmessers des strukturierenden Elementes bei der Dilatation, ergibt keine zusätzliche Information. Die obere Abbruchbedingung wurde programmbedingt vorgegeben und wirkte sich bei jeder Methode unterschiedlich aus. Die Abb. 5.2 a und b zeigt die Ergebnisse mit und ohne Berücksichtigung der Cutoffs am Beispiel eines mit Boxcounting gemessenen Einzelbildes der Vergrößerungsstufe 500x.

\subsubsection{Bestimmung der Cutoffs für Boxcounting}

Zunächst wurde für jedes nach dem Boxcounting-Verfahren gemessene Einzelbild ein log$\log$ Plot erstellt und mittels linearer Regression eine Gerade für alle Wertepaare angepasst. Dann wurde am unteren Skalenende das erste, die ersten beiden, die ersten drei usw. Wertepaare herausgenommen und jeweils der Korrelationskoeffizient bestimmt. Es wurde der Wertebereich berücksichtigt, für den der Korrelationskoeffizient das Optimum erreicht (s. Abb. 5.3). Auf Grund der negativen Steigung ist der Korrelationskoeffizient negativ. Für den oberen Cutoff wurden die Boxen so lange vergrößert, bis dreimal hintereinander die gleiche Anzahl Boxen gezählt wurde. 


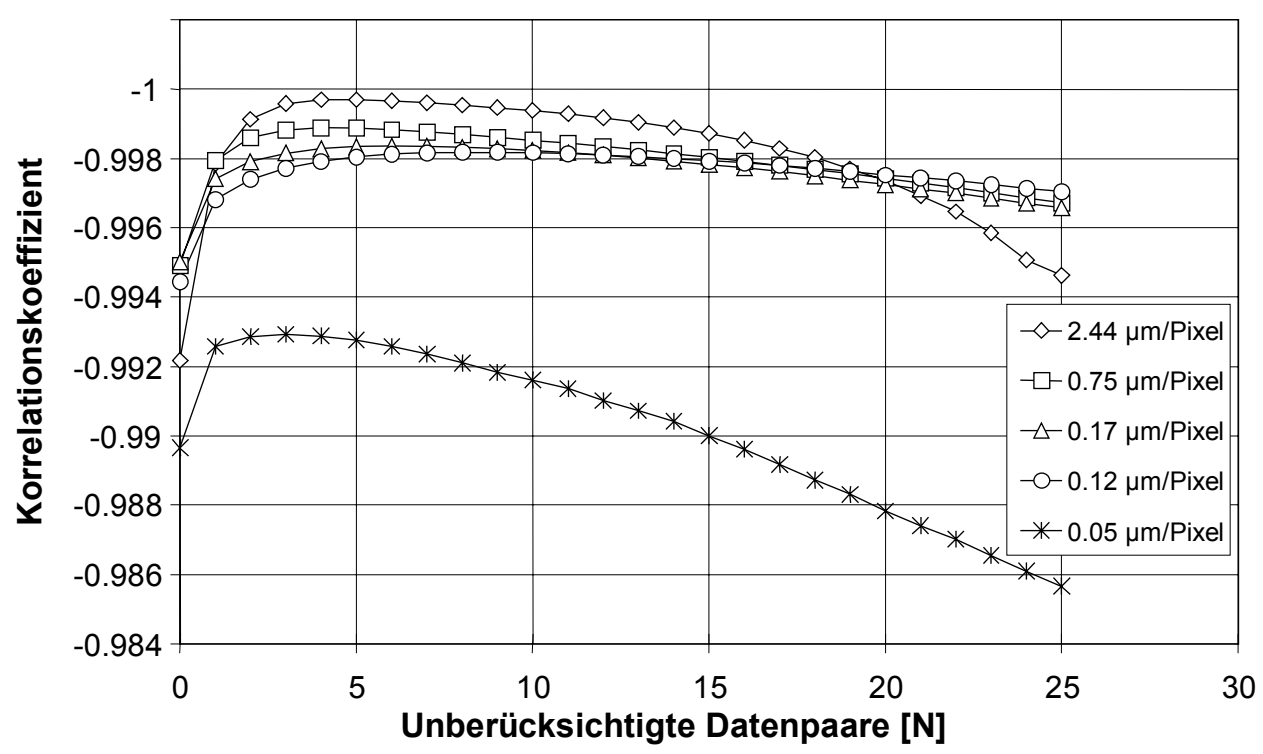

Abb. 5.3: Bestimmung des unteren Cutoffs für Boxcounting am Beispiel der Serie Leo8, für jeden Messschritt gemittelte Daten. Indem eine wachsende Anzahl von Datenpaaren im Richardson Plot nicht berücksichtigt wird, ändert sich der Korrelationskoeffizient für die entsprechende lineare Regression. ,0' auf der Abszisse steht für den gesamten Datensatz, ,1' bedeutet, das untere PixelWertepaar, das mit der Auflösung von einem Pixel aufgenommen wurde, wird herausgenommen usw. in Schritten von je zwei Pixeln.

\subsubsection{Bestimmung der Cutoffs für Dilatation}

Für Dilatation war das oben beschriebene Verfahren zur Bestimmung des unteren Cutoff nicht anwendbar, da der Korrelationskoeffizient mit jedem weggelassenen Datenpaar auf eine bessere Anpassung hinweist. Dies ist darauf zurückzuführen, dass die Messwerte im unteren Teil der Geraden (für große Strukturelemente) nicht streuen wie beim Boxcounting-Verfahren (s. Abb. 5.3 c). So wurden die jeweils ersten fünf Datenpaare nicht berücksichtigt, was ein in der Literatur gängiges Verfahren darstellt (z. B. Hamblin \& Stachowiak, 1994). Für die höchste Vergrößerungsstufe (5000x) wurde der Differenzenquotient $(\Delta \log$ Länge) $/(\Delta \log$ Skala)) berechnet (s. Abb. 5.4). Als Kriterium für den unteren Cutoff ist es sinnvoll, die 2. Ableitung (bzw. den entsprechende Differenzenquotienent) zu berechnen und für diese eine zulässige Abweichung von $0 \mathrm{zu}$ definieren, z.B. $\pm 0,025$, s. Markierung in Abb. 5.4. Für beide Methoden, Boxcounting und Dilatation, ergab sich so ein ähnlicher Bereich für den unteren Cutoff pro Einzelbild.

Als oberer Cutoff wurde die Umhüllende so lange dilatiert, bis $1 / 3$ des maximalen Feret (1/3 des Durchmessers eines flächengleichen Kreises des größten Objekts) oder 1/3 der schmalen Bildseite (s. Kaye 1989) erreicht war. 


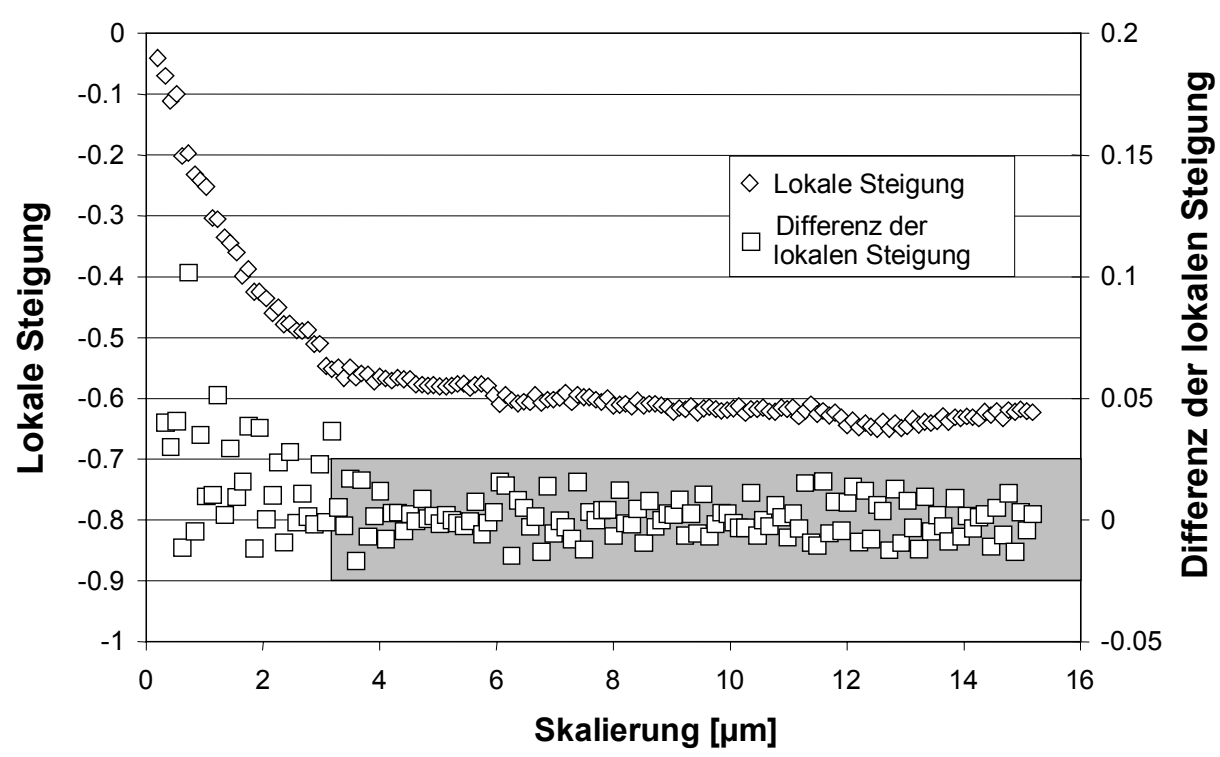

Abb. 5.4: Bestimmung des unteren Cutoffs für Dilatation am Beispiel eines mit der höchsten Auflösung aufgenommenen Einzelbildes. Abgebildet sind die Steigungen von Punkt zu Punkt und ihre Differenzen. Für die Berechnung der fraktalen Dimension wurden nur die Daten berücksichtigt, deren Differenzen der Steigung innerhalb eines bestimmten Intervalls, hier \pm 0.025 (grau markierter Bereich), liegen.

\subsubsection{Berechnung der Mittelwerte mit den Methoden , $\mathrm{A}^{\text {“6 }}$ und „, $\mathrm{B}$ “}

Zunächst wurde jedes Bild für boxcounting und Dilatation einzeln ausgewertet und der untere Cutoff bestimmt. Beim Vergleich der so ermittelten D zeigte sich, dass die Werte in Abhängigkeit von der betrachteten Spanne der Skalierungselemente eine systematische Drift aufweisen. Daher wurde für jede Vergrößerungsstufe und Schliff ein identischer Skalenbereich festgelegt, wodurch ein direkter Vergleich zwischen beiden Messverfahren und Verfahren der Mittelwertbildung möglich war. Der für das Boxcounting-Verfahren für eine Vergrößerungsstufe am häufigsten vorkommende Skalenbereich vom unteren bis zum oberen Cutoff wurde für alle Bilder auch des Dilatations-Verfahrens verwendet. Während die Größe des skalierenden Elementes des unteren Cutoff bei beiden Meßmethoden gut übereinstimmte, wurde mit der Dilatation ein deutlich weiterer Bereich in Richtung größerer Skalierungselemente abgedeckt. Für die Mittelwertbildung wurden nicht immer alle Bilder berücksichtigt, da bei einigen der obere Cutoff, vor allem für Boxcounting, nach relativ wenigen Messschritten erreicht worden war.

Die Berechnung der Mittelwerte und der Standardabweichung der mit Boxcounting und Dilatation gemessenen D einer Vergrößerungsstufe und eines Skalierungsbereichs erfolgte nach zwei unterschiedlichen Methoden.

- Für jede Vergrößerungsstufe eines Schliffs wurde die fraktale Dimension D für jedes einzelne Bild bestimmt und die D gemittelt (so viele Richardson Plots wie Bilder, Methode A) und

- die Rohdaten aller Bilder einer Vergrößerungsstufe eines Schliffes wurden gemittelt, was einem großen Bild entspricht, das aus zufällig verteilten Einzelbildern besteht (ein Richardson Plot pro Vergrößerungsstufe, Methode B). Für Methode B liegt für jede Datenmatrix eine umfangreiche Daten- und Varianzanalyse vor (s. Abb. 5.5). 


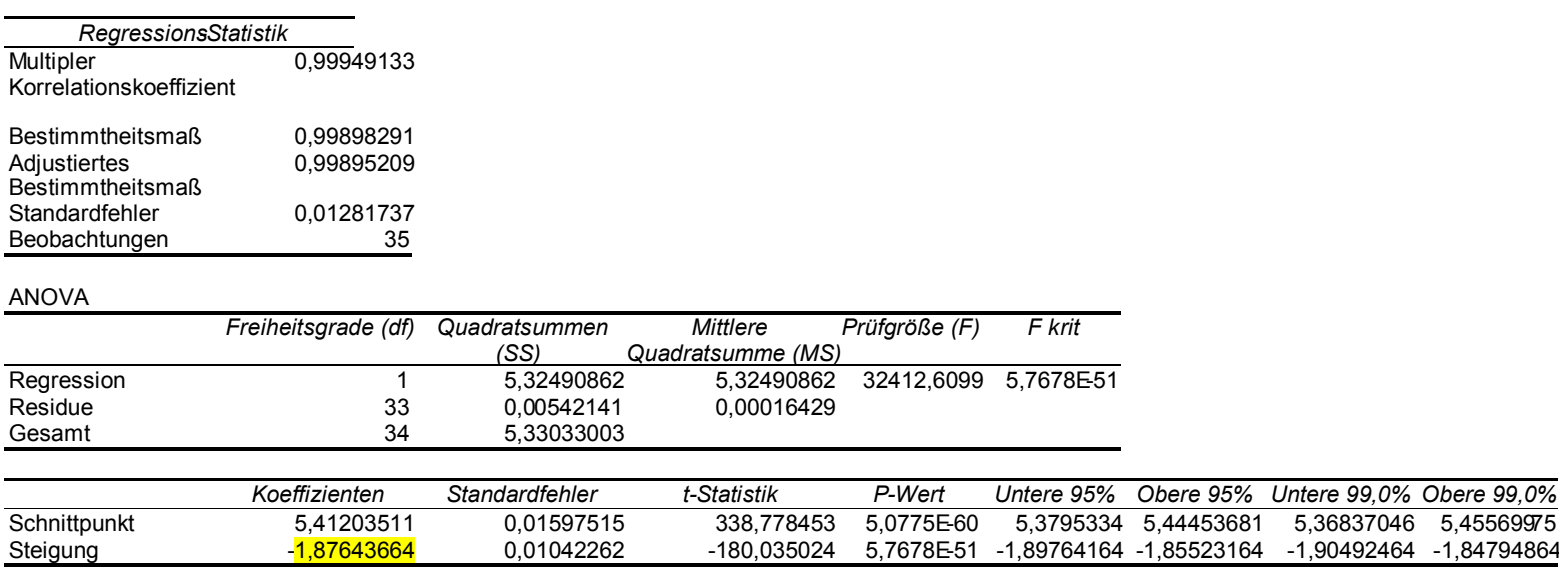

Abb. 5.5: Datenblatt für die lineare Regression nach Methode B (Mittelwerte einer Vergrößerungsstufe). Das Beispiel zeigt die Ergebnisse für 13 Bilder des Dünnschliffs Leo8, 500x Vergrößerung. Die fraktale Dimension (s. Markierung) ist der Betrag der Steigung der Regressionsgeraden, hier für die Daten des Boxcounting-Verfahrens angegeben.

Während sich für die Methode A die Standardabweichung sd (standard deviation) nach

$$
\mathrm{sd}=\sqrt{\sigma^{2}}=\sqrt{\left(\frac{1}{\mathrm{n}-1} \sum_{i}\left(\mathrm{x}_{\mathrm{i}}-\overline{\mathrm{x}}\right)^{2}\right)}
$$

auf die Varianz $\sigma^{2}$ zwischen den jeweiligen Einzelbildern bezieht, wird bei der in Abb. 5.5 gezeigten linearen Regression der Standardfehler se (standard error) der Steigung (hier rund 0,0104) angegeben. Der Standardfehler des Regressionskoeffizienten für die lineare Regression

$$
\mathrm{Y}_{i}=\hat{\beta} \mathrm{x}_{i}+\hat{\alpha}
$$

berechnet sich für den Schätzer der Steigung $\hat{\beta}$ nach

$$
\operatorname{se}^{(\hat{\beta})}=\frac{\hat{\sigma}}{\sum_{i}\left(x_{i}-\bar{x}\right)^{2}} .
$$

Dabei ist $\hat{\sigma}$ die Wurzel der Schätzung der Varianz $\hat{\sigma}^{2}$ und entspricht somit einer Standardabweichung. Die Schätzung der Varianz der gesamten linearen Regression bezieht sich auf die quadrierten Residuen

$$
\hat{\sigma}^{2}=\frac{1}{\mathrm{n}-2} \sum_{i}\left[\mathrm{y}_{\mathrm{i}}-\left(\hat{\alpha}+\hat{\beta} \mathrm{x}_{\mathrm{i}}\right)\right]^{2} .
$$

Somit wird für den Standardfehler der Steigung (Gl. 5.5) die Standardabweichung der gesamten linearen Regression auf die Quadratsumme der Abweichungen in x-Richtung bezogen (z. B. Stahel 1999). Dies kann nicht direkt mit den für Methode A erhobenen 
Standardabweichungen verglichen werden. In Gl. 5.3 bezieht sich das n auf die Anzahl der verwendeten Bilder, die Größe $\mathrm{x}_{\mathrm{i}}$ ist die fraktale Dimension der Einzelbilder. Wie in Kapitel 6.1.1 gezeigt wird, macht sich bei der Berechnung des Standardfehlers die Spanne der betrachteten Skalierung und die Anzahl der verwendeten Datenpaare bemerkbar.

\subsubsection{Anpassung der Daten verschiedener Vergrößerungsstufen eines Schliffs}

Werden die Richardson Plots aller Vergrößerungsstufen in einem Diagramm dargestellt, erscheinen die Geraden parallel verschoben (s. Abb. 5.6). Wie in Kapitel 5.1 beschrieben, stellt der y-Achsenabschnitt c die logarithmierte Anzahl der Boxen dar, die bei der Größe des skalierenden Elementes von $1 \mu \mathrm{m}$ die Konturlinie beinhalten würden. Diese Anzahl kann nicht größer als die Summe aller Pixel eines fiktiven Bildes der entsprechenden Auflösungsstufe werden. Daher ist c eine Maßzahl für die verwendeten Auflösungs- bzw. Vergrößerungsstufe.

Um eine fraktale Dimension über alle Vergrößerungsbereiche $\mathrm{zu}$ erhalten, wurden die Daten von Methode B bezüglich der Achsenabschnitte so angepasst, dass sich für jeden untersuchten Dünnschliff ein kontinuierlicher Datensatz ergab. Dafür wurde, beginnend mit der zweitgeringsten Auflösungsstufe $(0,75 \mu \mathrm{m} /$ Pixel), der gesamte Datensatz in yRichtung auf die Höhe der Regressionsgeraden der nächstgeringeren Auflösungsstufe (2,44 $\mu \mathrm{m} /$ Pixel) verschoben. Der verwendete Summand wurde für die höchste Boxgröße der zweiten Vergrößerungsstufe bestimmt und dieser Wert für den gesamten Datensatz verwendet.

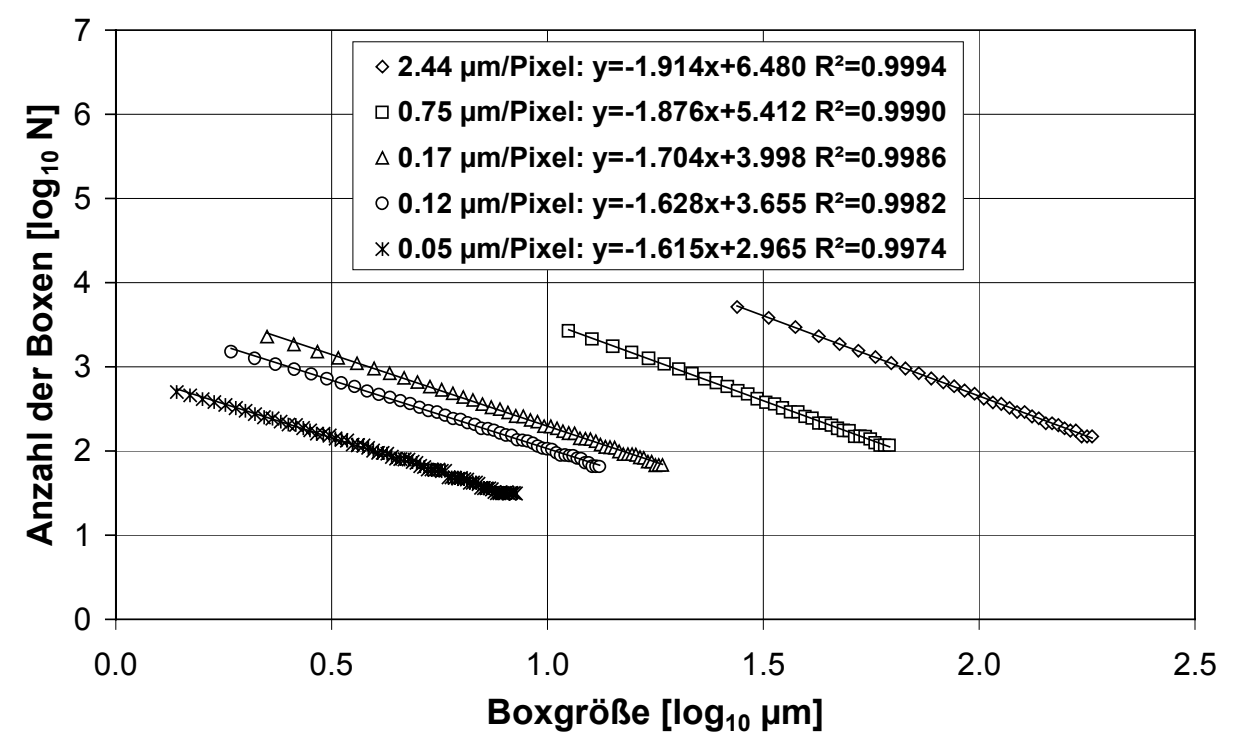

Abb. 5.6: Ergebnisse des Boxcounting-Verfahrens für die verschiedenen Auflösungsstufen innerhalb der unteren und oberen Cutoffs. Es handelt sich um die mit der Methode B gemittelten Daten des Schliffes Leo8. Die in den Regressionsgleichungen angegebenen Beträge der Steigungen entsprechen den fraktalen Dimensionen der jeweiligen Vergrößerungsstufe. $\mathrm{R}^{2}$ ist der quadrierte Regressionskoeffizient.

Das Verfahren wurde sukzessiv für die Datensätze der nächsthöheren Vergrößerungsstufen durchgeführt. Die Abb. 5.7 zeigt das Ergebnis für die in Abb. 5.6. dargestellten Datensätze. 


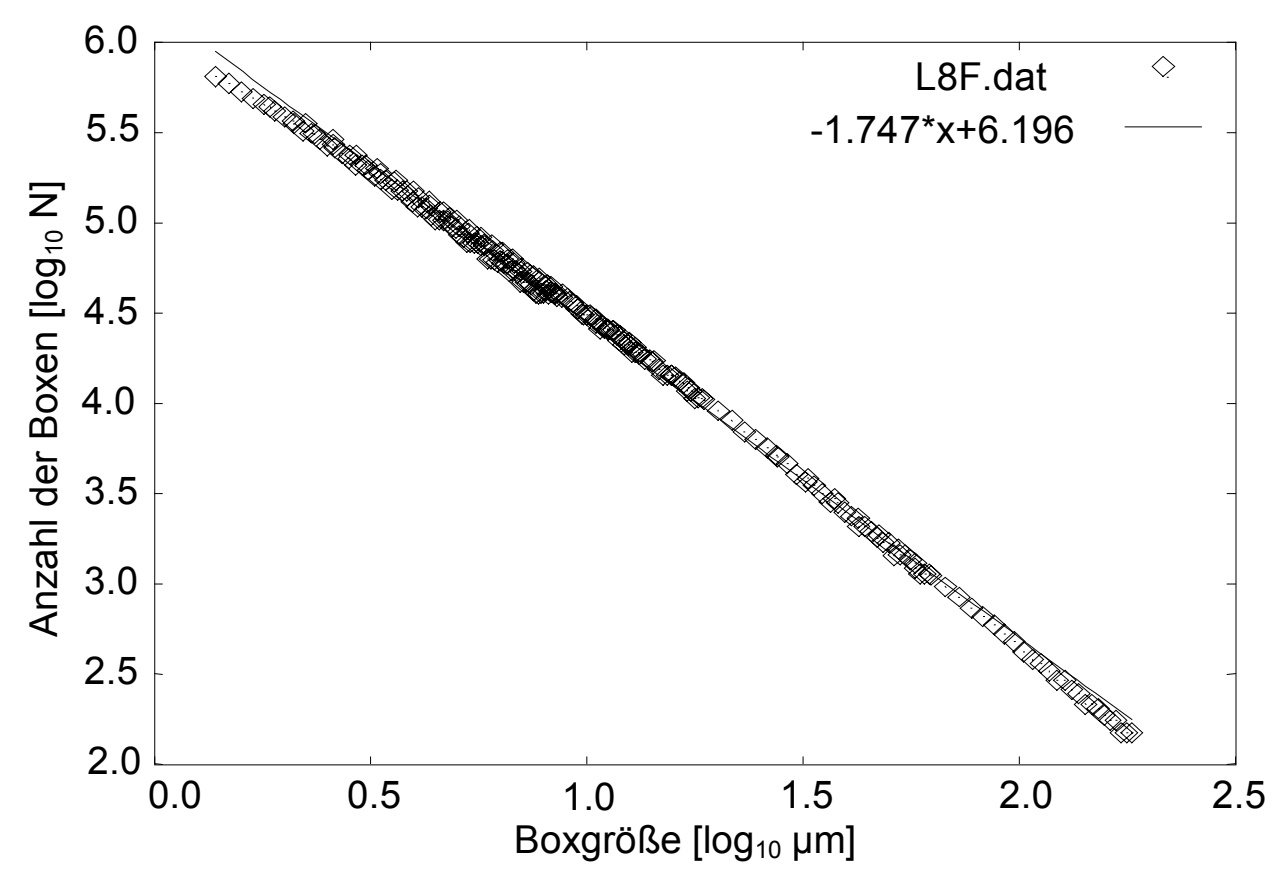

Abb. 5.7: Alle Vergrößerungsstufen des Schliffes Leo8, nach der Methode B gemittelte und bezüglich des y-Achsenabschnitts angepasste Messdaten der Boxcounting-Methode. Die mit einem robusten Schätzverfahren (lokale M-Schätzer, s. Kapitel 5.1.5) berechnete Regressionsgleichung ist angegeben.

\subsubsection{Lineare und exponentielle Schrittweiten}

Die von den Makros vorgegebenen Schrittweiten waren linear, was in logarithmischer Darstellungsweise zu einer Häufung der Daten in Richtung größerer strukturierender Elemente führte (s. Abb. 5.2 a). Bei der Berechnung der fraktalen Dimension durch lineare Regression und einer Optimierung durch die kleinsten Abweichungsquadrate führte dies zu einer stärkeren Gewichtung des Bereichs mit der höheren Datendichte. Gleichzeitig zeigte sich, dass D mit wachsender Vergrößerungsstufe bzw. Auflösung abnahm (s. die Steigung in den Regressionsgleichungen in Abb. 5.6). Diese beiden Effekte summierten sich. Durch die höhere Datendichte im steileren Teil des Richardson Plots wurde D leicht überschätzt.

Die Tatsache, dass D mit wachsender Auflösung abnahm, entsprach nicht den vorher angestellten Überlegungen. Daher wurde zusätzlich der Einfluß der Schrittweiten auf die fraktale Dimension untersucht. Eine Möglichkeit war die Umrechnung der Skalierungsschritte in exponentielle Schrittweiten, was zu gleichen Abständen der Daten im logarithmischen Plot führte. Dies erfolgte für die angepassten Daten (s. Kapitel 5.1.3) der Methode B mit einem $\mathrm{C}^{++}$-Programm. Die gewünschte exponentielle Schrittweite konnte vorgegeben werden, und der entsprechende Messwert (die Anzahl der Boxen bzw. die Länge der Umhüllenden) wurde aus den für dieses Intervall vorliegenden Daten arithmetisch gemittelt.

\subsubsection{Anpassung von Regressionsgeraden mit lokalen M-Schätzern}

Eine andere Ursache könnte sein, dass die Annahme einer einheitlichen fraktalen Dimension über den gesamten Skalenbereich unzutreffend ist. Um Fehlerquellen, wie 
durch nicht normalverteilte Daten verursacht, auszuschließen, erfolgte die Berechnung der Regressionsgeraden für die kontinuierlichen Daten aller Vergrößerungsstufen mit einer robusten Technik. Verwendet wurde ein Verfahren, das lokale M-Schätzer (MaximumLikelihood-Schätzungen für langschwänzige bzw. nicht-normalverteilte Verteilungen) und die Minimierung der absoluten Abweichung (statt der Abweichungsquadrate) zur Optimierung benutzt (Press et al. 1992). Für eine lineare Regression wird zunächst die Ausgleichsgerade nach der Methode der kleinsten Abweichungsquadrate berechnet. Die Steigung dieser Geraden wird dann schrittweise in beide Richtungen verändert und jeweils die Summe der absoluten Abweichungen (Summe der Beträge der Residuen) berechnet. So tragen Extremwerte nicht so sehr zur Anpassung bei. Auf diese Weise sollte zum einen die bereits erwähnte stärkere Gewichtung von Bereichen mit einer höheren Datendichte vermieden werden. Zum anderen wurde das Verfahren benutzt, um zwei Regressionsgeraden anzupassen. Dabei wurde mit sechs Datenpaaren für die erste Gerade begonnen, und der gemeinsame Datenpunkt sukzessive nach rechts in Richtung größerer Skalierungselemente verschoben, bis die zweite Gerade nur noch aus sechs Datenpaaren bestand.

Als Ergebnis wurden jeweils zwei Regressionsgleichungen und die entsprechende absolute Abweichung ausgegeben. Die Boxgröße, bei der die Anpassung die geringste absolute Abweichung (Summe der Beträge der Residuen) aufwies, wurde als optimaler Trennpunkt für beide Geraden angesehen (s. Abb. 5.8). Der Schnittpunkt zwischen den Geraden wurde aus den Regressionsgleichungen berechnet. Er stellt ein genaueres Maß für den Punkt dar, an dem in zwei Steigungen differenziert wird, als der gemeinsame Datenpunkt an dem die Trennung in zwei Geraden erfolgte. Der letztgenannte kann durch die Messung und bedingt durch das Addieren der Achsenabschnitte der verschiedenen Vergrößerungsstufen mit Fehlern behaftet sein.

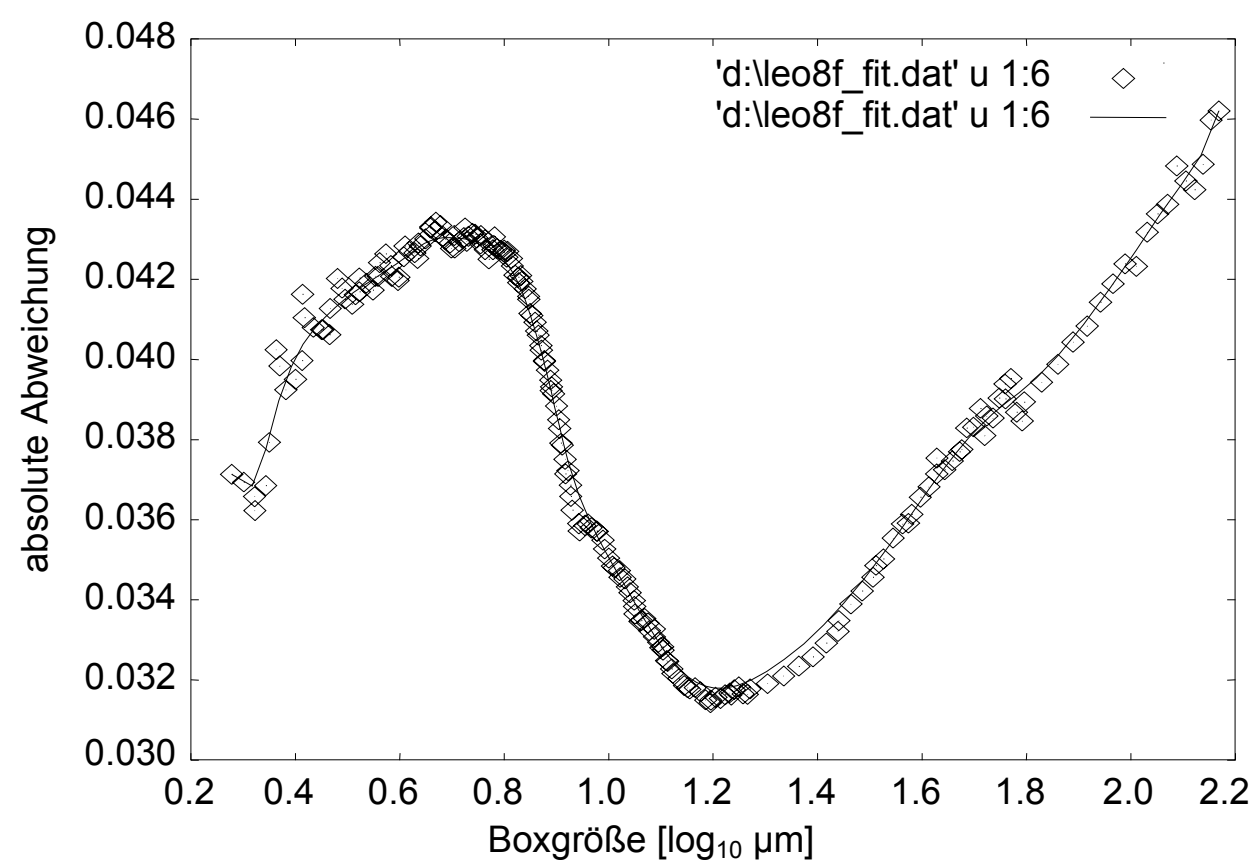

Abb. 5.8: Absolute Abweichung für die sukzessive Anpassung von zwei Regressionsgeraden für das Boxcounting-Verfahren und den Schliff Leo8 (s. Abb. 5.7). Die Boxgröße, bei der die Abweichung am geringsten ist, wird als Trennpunkt zwischen beiden Geraden festgesetzt. 
Die Abb. 5.9 zeigt die optimale Anpassung für Leo8, Boxcounting, für zwei Regressionsgeraden. Die Schnittpunkte für alle Messserien sind im Ergebnisteil (s. Kap. 6.1.1. und 6.1.2) tabellarisch dargestellt.

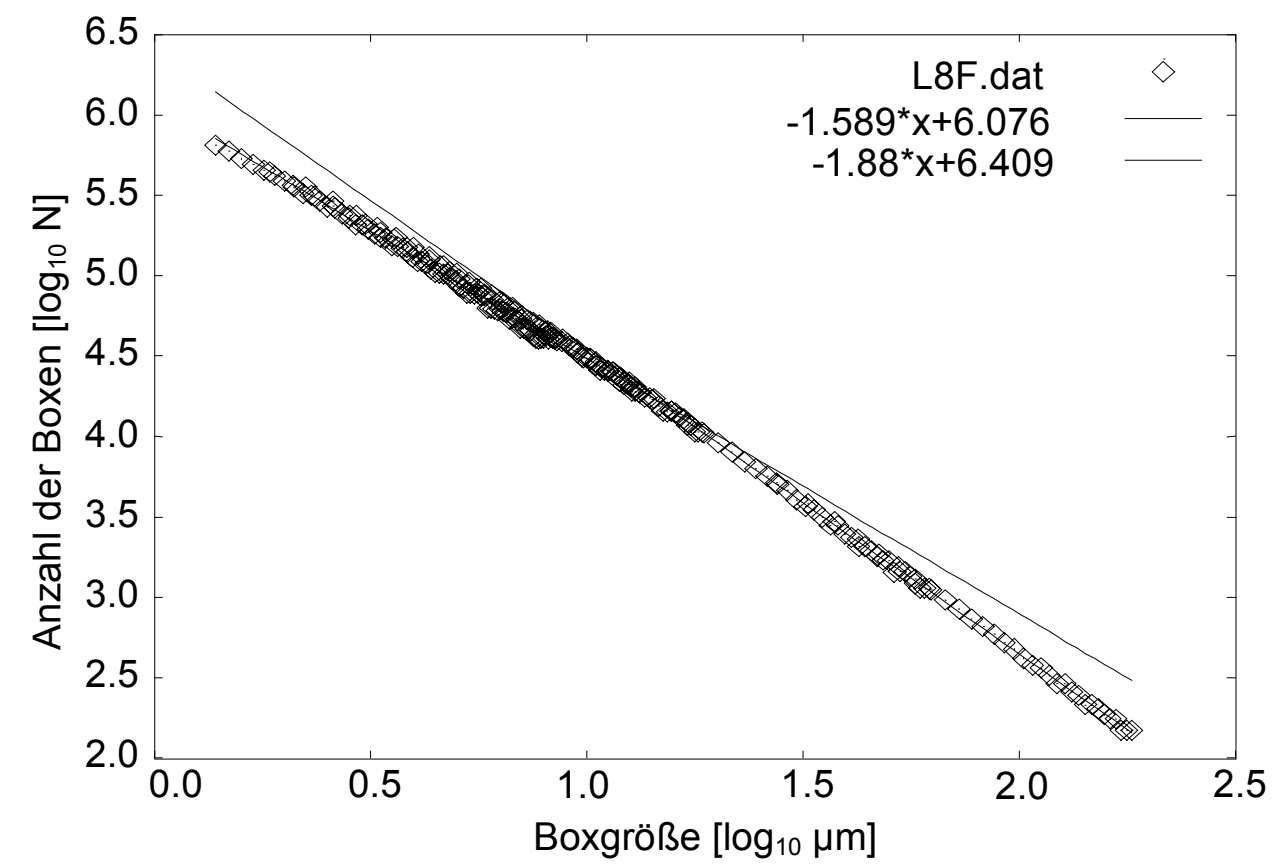

Abb. 5.9: Anpassung von zwei Geraden an die in Abb. 5.7 gezeigten Daten des Schliffes Leo8. Die Regressionsgleichungen sind angegeben.

\subsection{Parameterschätzung für die Abhängigkeit der fraktalen Dimension von der Auflösung}

Es wurde eine empirische Funktion erarbeitet (s. Kapitel 6.2), die die Abhängigkeit der gemessenen fraktalen Dimension von der Vergrößerungsstufe bzw. der Auflösung der REM-Aufnahmen beschreibt. Zwischen der Auflösung und dem mittleren Cutoff (dem logarithmischen Mittel zwischen unterem und oberem Cutoff der jeweiligen Vergrößerungsstufe) konnte ein linearer Zusammenhang aufgezeigt werden. Für die Parameterschätzung wurde das Programm ModelMaker (Version 3.0.3, Walker 1994) verwendet. Die Funktion, die als Variable die Auflösung und als Parameter die minimale und die maximale fraktale Dimension sowie einen Anpassungsparameter enthält, wurde vorgegeben. Die Parameter wurden geschätzt, indem die Funktion mit dem LevenbergMarquardt Verfahren an die vorhandenen Messwerte angepasst wurde $\left(\mathrm{D}_{\mathrm{L}}\right.$ in Tab. 6.1-3). Dieses Verfahren wurde für jeden Schliff und die beiden verwendeten Methoden gesondert durchgeführt.

\subsection{Berechnung der Porengrößenverteilung}

Zunächst wurden die Rohdaten für jeden Schliff und jedes der 19 bis 21 pro Auflösungsstufe vorliegende FE-REM Bildern gemittelt. Bei den lichtmikroskopischen Bildern erfolgte die Auswertung analog für beide Schliffrichtungen. Die Porendurchmesser ergaben sich aus dem Durchmesser des Strukturelements (s. Kapitel 4.6.4). Es wurde die 
distributive und die kumulative Porengrößenverteilung (bzw. die Dichte und die Verteilung der Porengrößenklassen) in Porenfläche pro Bildfläche in Prozent berechnet. Für die distributive Porengrößenverteilung wurde für jede Porenklasse die Standardabweichung und der Variationskoeffizient cv (coefficient of variation) in Prozent

$$
\mathrm{cv}(\mathrm{x})=\frac{\mathrm{sd}(\mathrm{x})}{\overline{\mathrm{x}}} \cdot 100
$$

aus dem Quotienten aus Standardabweichung und Mittelwert bestimmt. „Große“ Poren im Sinne von „größte mit der jeweiligen Vergrößerungsstufe erfasste Poren“ existierten nur für wenige Bilder pro Messserie, nach der Erfassung der größten Pore brach das Bildverarbeitungsprogramm ab. Für jeden Datensatz wurde zusätzlich zur kleinsten und größten erfassten Porenklasse der Durchmesser markiert, bei dem die entsprechende Porengröße bei der Hälfte bzw. bei allen Bildern vorkam, und bei dem cv $<100 \%$ wurde. Letzteres lag immer ungefähr in dem Bereich, in dem auch alle Bilder besetzt waren (s. Tab. A.1 und A.2).

Die für die verschiedenen Auflösungsstufen gemessenen Porengrößenverteilungen lassen sich nicht unmittelbar vergleichen. Die Prozentangaben (Porenfläche/Bildfläche $\cdot 100$ ) beziehen sich zusätzlich auf die verwendete Schrittweite des skalierenden Elements, die gleichbedeutend ist mit der Breite der jeweils betrachteten Porenklasse. Um die distributive Porenhäufigkeit aller Vergrößerungsstufen der FE-REM Aufnahmen zu erhalten, wurde die prozentuale Porenfläche pro Opening Schritt auf die jeweilige Schrittweite des skalierenden Elements bezogen. Dies erfolgte gesondert für die verschiedenen Vergrößerungsstufen der Dünnschliffe Leo7 und Leo8 (s. Abb. 6.20 und 6.21).

\subsubsection{Summierung der Porengrößenverteilung verschiedener Vergrößerungsstufen}

Die kumulierten Porengrößenverteilungen für die einzelnen Vergrößerungsstufen waren ähnlich wie in den Richardson Plots (s. Abb. 5.6) - parallel verschoben (s. Abb. 5.10 a). Der Grund ist darin zu sehen, dass Poren, die kleiner als die jeweilige Bildauflösung sind, nicht erfasst werden können. Daher wurden die Flächenanteile von den kleinsten erfassten Poren her aufsummiert, wobei die Durchmesser entsprechend der Kapillarengleichung (Gl. 2.20) in die entsprechende Wasserspannung umgerechnet werden können.

Dies erfolgte jeweils genau an dem Punkt, an dem der Variationskoeffizient der höheren Vergrößerungsstufe kleiner als $100 \%$ wurde. Da auf Grund der unterschiedlichen Auflösungen kein Messwert für den exakt gleichen Skalierungsschritt der nächst niedrigeren Vergrößerungsstufe vorhanden war, wurde dort der entsprechende kumulierte Flächenanteil zwischen den zwei benachbarten Skalierungsschritten linear interpoliert. Der so ermittelte Summand wurde für den gesamten Datensatz der niedrigeren Vergrößerungsstufe verwendet und mit den folgenden Vergrößerungsstufen analog verfahren (s. auch Kap. 5.1.3), so dass der Variationskoeffizient der niedrigsten Stufe für die Addition keine Rolle spielte. 

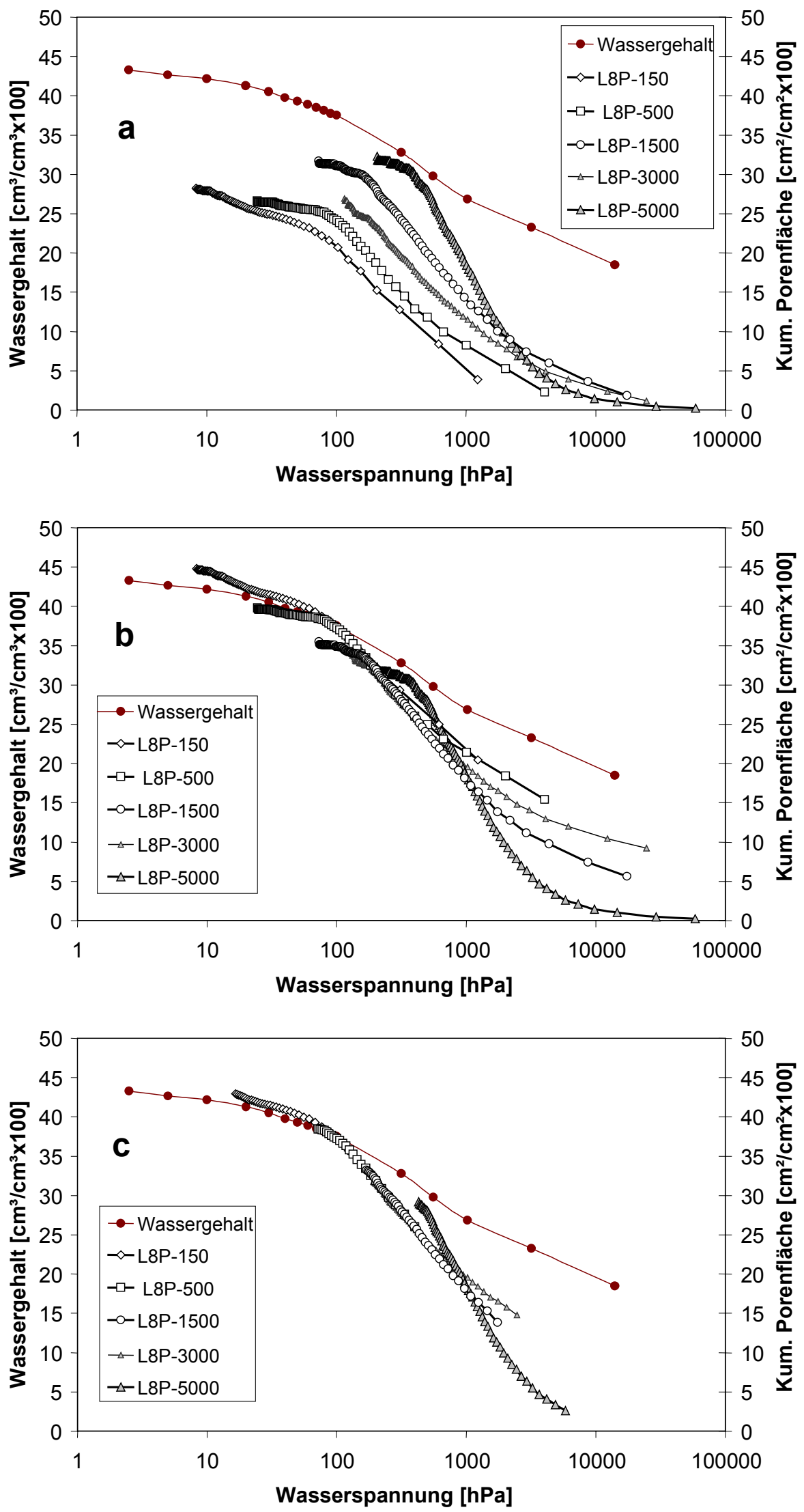

Abb. 5.10: Vergleich der Porengrößenverteilungen für Leo8 mit der am selben Bodenhorizont aufgenommenen Retentionskurve. (a) Einzeln dargestellt erscheinen die Kurven parallel verschoben; (b) Aufsummierung und Darstellung aller Werte; (c) Darstellung ohne die ersten fünf Messwerte für die jeweils kleinsten Poren bis zu der Größenklasse, die auf mindestens 50 \% aller Bilder vorkommt. 
Voruntersuchungen hatten ergeben, dass bei dem gewählten Verfahren die Datensätze im jeweils „sicheren“ Bereich summiert werden, d. h. der Punkt, ab dem die Daten verkettet wurden, weist für beide Vergrößerungsstufen einen $\mathrm{cv}<100 \%$ auf. In Abb. 5.10 b und c sind die kumulierten Porengrößenverteilungen von Leo8 für unterschiedliche Bereiche dargestellt.

Für die visuelle Darstellung bot sich die in Abb. 6.18 und 6.19 gezeigte Variante an: In Richtung großer Poren wurden nur die Größenklassen berücksichtigt, die zu mindestens $50 \%$ besetzt sind. Bei der niedrigsten Vergrößerungsstufe wurden alle Werte dargestellt, in diesem Bereich war es legitim, anzunehmen, dass relativ große Poren nicht in jedem Bildausschnitt erscheinen können. Die 5 Werte im Bereich der jeweils kleinsten erfassten Poren wurden aufgrund der Pixelrepräsentanz und weil sich die Messbereiche überlappen nicht abgebildet. Für die höchste Vergrößerung wurden sie dargestellt, um den unteren Ast der Kurve zeigen zu können.

\subsection{Parametrisierung der im Labor ermittelten Retentions- und Leitfähigkeitskurven}

Die Parametrisierung der Retentionskurve erfolgt üblicherweise in Hinblick auf die Modellierung der Wassertransportgleichung, die in der in G1. 2.24 angegebenen Form den Wassergehalt und die Wasserspannung sowie die Wasserleitfähigkeit beinhaltet. In der vorliegenden Arbeit erfolgte die Anpassung unter dem Gesichtspunkt, das hydraulische Verhalten des untersuchten Bodenhorizonts $\mathrm{zu}$ charakterisieren. Dies beinhaltet den Vergleich zwischen der bildanalytisch gemessenen Porengrößenverteilung, da der Parameter $\lambda$ der Anpassung nach Brooks \& Corey (1964) auch als Porengrößenindex beschrieben wird und der Parameter n der Anpassung nach van Genuchten (1980) diesem Index invers proportional ist.

Die im Labor unter statischen Bedingungen aufgenommene Retentionskurve für die 100 $\mathrm{cm}^{3}$ Proben aus $70 \mathrm{~cm}$ Tiefe wurde mit dem Optimierungsprogramm RETC (van Genuchten et al., 1991) parametrisiert. Dies erfolgte zum einen mit dem van Genuchten Modell (s. Gl. 2.29) unter der Annahme einer uni- und einer bimodalen Porengrößenverteilung (Durner 1991, Diekkrüger 1992). Es wurden jeweils Werte für den Wassergehalt bei Sättigung $\theta_{\mathrm{s}}$, den residualen Wassergehalt $\theta_{\mathrm{r}}$, und die Anpassungsparameter $\alpha$ und $n$ ermittelt. Für $\theta_{\mathrm{s}}$ wurde der gemessene Wert als Startwert der Optimierung verwendet (s. Friedrichs, 1999). Zum anderen wurde das Modell von Brooks and Corey (G1. 2.28) für die Parametrisierung der Retention verwendet. Es wurden Werte für $\theta_{\mathrm{s}}, \psi_{\mathrm{b}}$ (Wasserspannung beim Lufteintrittspunkt) und $\lambda$ angepasst.

Aus den Parametern der Anpassung nach van Genuchten wurden nach Mualem (1976, G1. 2.27 und 2.30) die Wasserleitfähigkeiten sowohl für die unimodale als auch für die bimodale Porengrößenverteilung berechnet und mit den im Labor gemessenen Leitfähigkeiten verglichen. Die Koeffizienten $\lambda$ und $n$ wurden mit den bildanalytisch gemessenen fraktalen Dimensionen in Beziehung gebracht.

Eine detaillierte Beschreibung des Programms kann, wie das Programm selbst, im Internet (University of California, Riverside, USA) unter

http://www.ussl.ars.usda.gov/MODELS/MODELS.HTM. abgerufen werden. 


\section{Ergebnisse}

Die Darstellung der Ergebnisse wird nach den verschiedenen Messverfahren gegliedert und umfaßt im Wesentlichen die bildanalytische Messung der (i) fraktalen Dimension und (ii) der Porengrößenverteilung einschließlich der mathematischen und statistischen Auswertung. Ergebnisse der Simulationsansätze schließen sich an. Eine Methodik zur Auswertung der Daten im Sinne eines Lehrbuchverfahrens stand nicht zur Verfügung. Die angewendeten Verfahren beruhen auf den Erfahrungen anderer Autoren bzw. wurden selbst entwickelt wurden. Zur Verdeutlichung der Vorgehensweise wurde ein Teil der Ergebnisse als Abbildungen bereits in Kapitel 5 vorgestellt.

\subsection{Die fraktale Dimension der Porenraumgrenzlinie}

Im Folgenden werden die Ergebnisse in rasterelektronenmikroskopische (REM) und lichtmikroskopische (LM) Aufnahmen unterteilt. Bedingt durch die Aufnahmetechnik, insbesondere durch die unterschiedliche Tiefenschärfe, war der Unterschied der bildanalytisch gemessenen fraktalen Dimensionen (D) so groß, dass die Ergebnisse nicht, wie für die verschiedenen Vergrößerungsstufen der REM Aufnahmen, direkt verglichen werden konnten.

\subsubsection{Messungen an FE-REM Aufnahmen}

In den Tabellen 6.1 bis 6.3 werden die Ergebnisse für Leo7, Leo8 und Leo9 für die Methoden Boxcounting und Dilatation gezeigt. Hierbei ist der für Leo7 verwendete Schliff vertikal und der für Leo8 verwendete horizontal ausgerichtet und die im Abschnitt 6.1.2 dargestellten Ergebnisse der lichtmikroskopischen Aufnahmen von Dünnschliffen entstammen derselben Kubiena-Box wie Leo7 und Leo8. Der ebenfalls horizontal ausgerichtete Schliff Leo9 entstammt einer anderen Kubiena-Box als Leo7 und Leo8.

Als für die Ergebnisse relevante Größen sind jeweils die physikalische Auflösung, mit der die Bilder aufgenommen wurden, der untere und der obere Cutoff, die Anzahl der für die Auswertung verwendeten Bilder und die Anzahl der Datenpaare innerhalb der Cutoffs angegeben. Die Werte für D sind getrennt nach Methode A und B dargestellt. Die Angaben in Klammern sind die Standardabweichung (Methode A) und der Standardfehler der Steigung (Methode B).

Die Bildauflösung zur Charakterisierung der Vergrößerungsstufe wurde gewählt, weil die Angabe der Vergrößerungsstufe, wie sie die Software des verwendeten FE-REM liefert, vom jeweiligen als Standarddrucker eingerichteten Drucker abhängt. 
Tab. 6.1: Fraktale Dimensionen und für deren Berechnung relevante Parameter der Messserie Leo7. Methode A: $D_{L}$ als Mittelwert der einzelnen Bilder, $n(A)$ ist die Anzahl der Einzelbilder. Methode B: Berechnung von D aus den gemittelten Rohdaten, $n$ (B) ist die Anzahl der Datenpaare für jeden Datensatz. sd ist die Standardabweichung für die einzelnen Bilder untereinander und se der Standardfehler des Regressionskoeffizienten für die Steigung.

\begin{tabular}{|c|c|c|c|c|c|c|c|}
\hline Methode & $\begin{array}{c}\text { Auflösung[ } \\
\mu \mathrm{m} / \text { Pixel] }\end{array}$ & $\begin{array}{c}\text { unterer } \\
\text { Cutoff } \\
{[\mu \mathrm{m}]}\end{array}$ & $\begin{array}{c}\text { oberer } \\
\text { Cutoff } \\
{[\mu \mathrm{m}]}\end{array}$ & $n(A)$ & $\mathrm{n}(\mathrm{B})$ & $\begin{array}{c}\text { Methode A } \\
D_{\llcorner} \text {mit sd }\end{array}$ & $\begin{array}{l}\text { Methode } B \\
D_{\llcorner} \text {mit se }\end{array}$ \\
\hline $\begin{array}{c}\text { Box- } \\
\text { counting }\end{array}$ & \multirow{2}{*}{2,50} & \multirow{2}{*}{25,23} & \multirow{2}{*}{221,95} & 13 & 41 & $1,915(0,020)$ & $1,915(0,008)$ \\
\hline Dilatation & & & & 13 & 40 & $1,889(0,024)$ & $1,889(0,005)$ \\
\hline $\begin{array}{c}\text { Box- } \\
\text { counting }\end{array}$ & \multirow{2}{*}{0,75} & \multirow{2}{*}{8,91} & \multirow{2}{*}{54,07} & 17 & 31 & $1,901(0,017)$ & $1,900(0,010)$ \\
\hline Dilatation & & & & 17 & 31 & $1,864(0,022)$ & $1,864(0,007)$ \\
\hline $\begin{array}{c}\text { Box- } \\
\text { counting }\end{array}$ & \multirow[t]{2}{*}{0,21} & \multirow[t]{2}{*}{9,79} & \multirow[t]{2}{*}{19,17} & 20 & 23 & $1,820(0,060)$ & $1,821(0,033)$ \\
\hline Dilatation & & & & 20 & 23 & $1,788(0,057)$ & $1,788(0,002)$ \\
\hline $\begin{array}{c}\text { Box- } \\
\text { counting }\end{array}$ & \multirow[t]{2}{*}{0,12} & \multirow[t]{2}{*}{1,72} & \multirow[t]{2}{*}{14,51} & 15 & 53 & $1,616(0,053)$ & $1,616(0,011)$ \\
\hline Dilatation & & & & 15 & 53 & $1,571(0,050)$ & $1,571(0,008)$ \\
\hline $\begin{array}{c}\text { Box- } \\
\text { counting }\end{array}$ & \multirow[t]{2}{*}{0,05} & \multirow[t]{2}{*}{2,74} & \multirow{2}{*}{5,69} & 17 & 29 & $1,569(0,086)$ & $1,569(0,025)$ \\
\hline Dilatation & & & & 17 & 29 & $1,564(0,085)$ & $1,563(0,002)$ \\
\hline
\end{tabular}

Der untere und obere Cutoff wurde wie in Kapitel 5.1.1 beschrieben ermittelt. Die Anzahl der Einzelbilder stellt die unter Berücksichtigung der Cutoffs für die Auswertung verwendeten Bilder dar. Die Anzahl der Datenpaare ist die Anzahl der Messschritte. Bei Leo9 wurde die Dilatation mit doppelter Schrittweite durchgeführt, daher liegt hier nur ca. die Hälfte der Messschritte wie für das Boxcountingverfahren vor.

Das Hauptergebnis der bildanalytischen Messung der Porenraumgrenzlinie ist darin zu sehen, dass die fraktale Dimension mit wachsender Auflösung bzw. Vergrößerungsstufe abnimmt. Dies gilt für alle in den Tab. 6.1 bis 6.3 dargestellten Messserien. Im folgenden wird die mit Boxcounting gemessene fraktale Dimension mit $\mathrm{D}^{\mathrm{b}}$ und die mit Dilatation gemessene mit $\mathrm{D}^{\mathrm{d}}$ bezeichnet.

Die Abb. 6.1 und 6.2 zeigen analog zu Tab. 6.1 und 6.3 die gemittelten Daten (Methode B) der Serien Leo7 und Leo9 für das Boxcounting-Verfahren innerhalb der Cutoffs. Die entsprechende Abbildung für Leo8 befindet sich als Abb. 5.6 in Kapitel 5 „Mathematische Auswertung und Statistik“. 


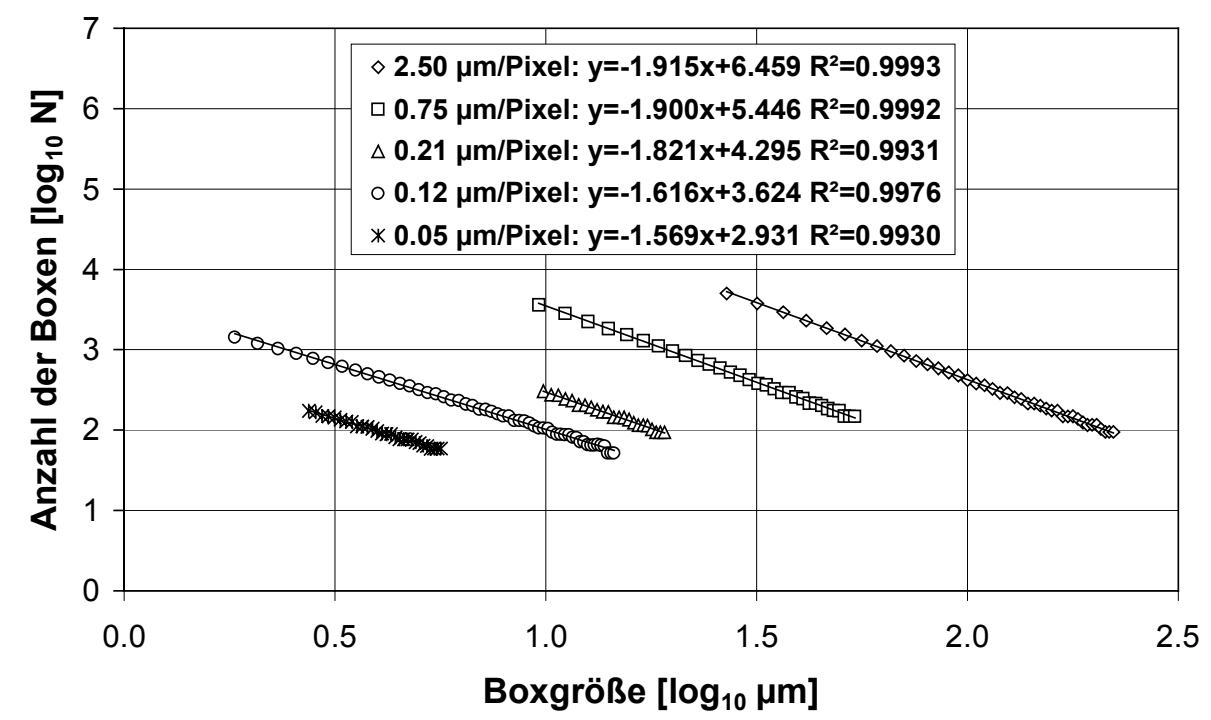

Abb. 6.1: Ergebnisse der Boxcounting-Methode für die verschiedenen Auflösungsstufen innerhalb des unteren und oberen Cutoff des Schliffes Leo7 (Methode B). Die in den Regressionsgleichungen angegebenen Steigungen entsprechen der fraktalen Dimension der jeweiligen Vergrößerungsstufe. $\mathrm{R}^{2}$ ist der quadrierte Regressionskoeffizient.

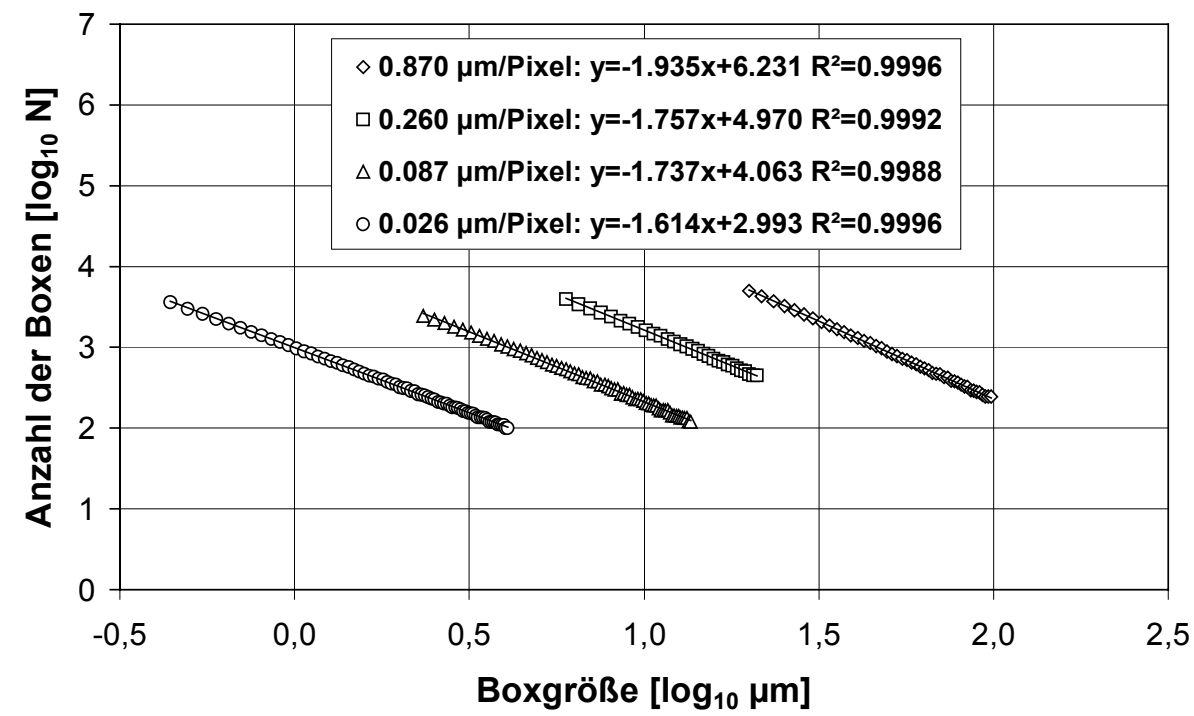

Abb. 6.2: Ergebnisse der Boxcounting Methode für die verschiedenen Auflösungsstufen innerhalb des unteren und oberen Cutoff des Schliffes Leo9 (Methode B, s. Abb. 6.1).

Die mit Boxcounting gemessenen $\mathrm{D}^{\mathrm{b}}$ sind immer etwas höher als die mit Dilatation $\mathrm{D}^{\mathrm{d}}$ gemessenen. Der Unterschied durch die Methode der Mittelwertbildung (Methode A oder Methode B) ist minimal und drückt sich vor allem in der durch die Standardabweichung (sd) bzw. den Standardfehler (se) beschriebenen Schwankungsbreite der D aus. Die Standardabweichung bezieht sich auf die Abweichung der an Einzelbildern gemessenen D untereinander, der Standardfehler auf die Abweichung der Steigung der an die gemittelten Rohdaten angepassten Regressionsgeraden. 
Tab. 6.2: Fraktale Dimensionen und für deren Berechnung relevante Parameter der Messserie Leo8 (s. Tab. 6.1)

\begin{tabular}{|c|c|c|c|c|c|c|c|}
\hline Methode & $\begin{array}{c}\text { Auflösung[ } \\
\mu \mathrm{m} / \text { Pixel] }\end{array}$ & $\begin{array}{c}\text { unterer } \\
\text { Cutoff } \\
{[\mu \mathrm{m}]}\end{array}$ & $\begin{array}{c}\text { oberer } \\
\text { Cutoff } \\
{[\mu \mathrm{m}]}\end{array}$ & $n(A)$ & $\mathrm{n}(\mathrm{B})$ & $\begin{array}{c}\text { Methode A } \\
D_{\llcorner} \text {mit sd }\end{array}$ & $\begin{array}{l}\text { Methode B } \\
D_{\llcorner} \text {mit sd }\end{array}$ \\
\hline $\begin{array}{c}\text { Box- } \\
\text { counting }\end{array}$ & \multirow{2}{*}{2,44} & \multirow{2}{*}{27,50} & \multirow{2}{*}{185,60} & 14 & 32 & $1,914(0,017)$ & $1,914(0,008)$ \\
\hline Dilatation & & & & 14 & 33 & $1,902(0,024)$ & $1,903(0,003)$ \\
\hline $\begin{array}{c}\text { Box- } \\
\text { counting }\end{array}$ & \multirow{2}{*}{0,75} & \multirow{2}{*}{10,52} & \multirow{2}{*}{61,94} & 13 & 35 & $1,876(0,031)$ & $1,876(0,010)$ \\
\hline Dilatation & & & & 13 & 35 & $1,845(0,031)$ & $1,845(0,007)$ \\
\hline $\begin{array}{c}\text { Box- } \\
\text { counting }\end{array}$ & \multirow{2}{*}{0,17} & \multirow{2}{*}{2,24} & \multirow{2}{*}{18,64} & 21 & 48 & $1,704(0,057)$ & $1,704(0,009)$ \\
\hline Dilatation & & & & 21 & 48 & $1,677(0,059)$ & $1,677(0,007)$ \\
\hline $\begin{array}{c}\text { Box- } \\
\text { counting }\end{array}$ & \multirow[t]{2}{*}{0,12} & \multirow[t]{2}{*}{1,85} & \multirow[t]{2}{*}{13,21} & 20 & 47 & $1,628(0,053)$ & $1,628(0,010)$ \\
\hline Dilatation & & & & 20 & 47 & $1,596(0,054)$ & $1,596(0,007)$ \\
\hline $\begin{array}{c}\text { Box- } \\
\text { counting }\end{array}$ & \multirow{2}{*}{0,05} & \multirow[t]{2}{*}{1,38} & \multirow{2}{*}{8,51} & 17 & 70 & $1,615(0,084)$ & $1,615(0,010)$ \\
\hline Dilatation & & & & 17 & 70 & $1,580(0,080)$ & $1,580(0,006)$ \\
\hline
\end{tabular}

Tab. 6.3: Fraktale Dimensionen und für deren Berechnung relevante Parameter der Messserie Leo9 (s. Tab. 6.1).

\begin{tabular}{|c|c|c|c|c|c|c|c|}
\hline Methode & $\begin{array}{c}\text { Auflösung[ } \\
\mu \mathrm{m} / \text { Pixel] }\end{array}$ & $\begin{array}{c}\text { unterer } \\
\text { Cutoff } \\
{[\mu \mathrm{m}]}\end{array}$ & $\begin{array}{c}\text { oberer } \\
\text { Cutoff } \\
{[\mu \mathrm{m}]}\end{array}$ & $\mathrm{n}(\mathrm{A})$ & $\mathrm{n}(\mathrm{B})$ & $\begin{array}{c}\text { Methode A } \\
D_{\llcorner} \text {mit sd }\end{array}$ & $\begin{array}{l}\text { Methode } B \\
D_{\llcorner} \text {mit se }\end{array}$ \\
\hline $\begin{array}{c}\text { Box- } \\
\text { counting }\end{array}$ & \multirow{2}{*}{0,870} & \multirow{2}{*}{18,66} & \multirow{2}{*}{98,26} & 11 & 46 & $1,935(0,011)$ & $1,935(0,006)$ \\
\hline Dilatation & & & & 11 & 23 & $1,911(0,014)$ & $1,911(0,006)$ \\
\hline $\begin{array}{c}\text { Box- } \\
\text { counting }\end{array}$ & \multirow{2}{*}{0,260} & \multirow{2}{*}{5,58} & \multirow{2}{*}{21,04} & 10 & 30 & $1,757(0,051)$ & $1,757(0,010)$ \\
\hline Dilatation & & & & 10 & 15 & $1,709(0,057)$ & $1,709(0,012)$ \\
\hline $\begin{array}{c}\text { Box- } \\
\text { counting }\end{array}$ & \multirow{2}{*}{0,087} & \multirow{2}{*}{2,22} & \multirow{2}{*}{13,59} & 11 & 66 & $1,737(0,023)$ & $1,736(0,007)$ \\
\hline Dilatation & & & & 11 & 32 & $1,698(0,020)$ & $1,698(0,009)$ \\
\hline $\begin{array}{c}\text { Box- } \\
\text { counting }\end{array}$ & \multirow{2}{*}{0,026} & \multirow{2}{*}{0,44} & \multirow{2}{*}{4,11} & 18 & 71 & $1,614(0,083)$ & $1,614(0,004)$ \\
\hline Dilatation & & & & 18 & 35 & $1,611(0,081)$ & $1,611(0,002)$ \\
\hline
\end{tabular}


Wie in den Tabellen 6.1 und $6.2 \mathrm{zu}$ erkennen ist wird durch die höchste Auflösungsstufe kaum noch ein „weiterer“ Messbereich für den unteren Cutoff im Sinne von besserer Auflösung erreicht. Bei Leo7 erreicht er nicht einmal die Auflösung des unteren Cutoffs der zweithöchsten Auflösungsstufe (s. Abb. 6.1). Bei Leo9 jedoch, wo die Bilder mit einer doppelt so hohen Auflösung aufgenommen wurden, wird ein deutlich weiterer Bereich in Richtung kleinerer Objekte abgedeckt (s. Tab. 6.3 und Abb. 6.3).

Bei der niedrigsten Vergrößerungsstufe von Leo7 und Leo8 stimmt die Anzahl der Datenpaare nicht exakt überein. Der Grund ist darin zu sehen, dass die Seitenlänge der Box aus der Anzahl der Boxen multipliziert mit der Auflösung und der Dicke der DilatationsUmhüllenden aus dem Quotienten von Fläche und Länge bestimmt wird. Gerade im Bereich einer geringen Auflösung stimmen die Skalierungsschritte nicht überein, was zu der beobachteten Abweichung führt.

Die Standardabweichungen nehmen im allgemeinen mit der Vergrößerungsstufe zu, wobei die zweithöchste Vergrößerungsstufe eine Ausnahme darstellt. Hier weisen die Standardabweichungen auf eine geringere Varianz der Einzelbilder untereinander hin. Besonders deutlich ist dies bei Leo9 (Tab. 6.3) wo die ursprünglich mit 3000x beschriebene Vergrößerungsstufe ganz weggelassen und bereits bei der Aufnahme der Bilder die Auflösung verdoppelt wurde (s. Tab. 4.1). Bei Leo7 und Leo8 ist dieser Sachverhalt nicht so ausgeprägt, der Trend aber dennoch erkennbar (vergleiche Tab. 6.1 und 6.2).

Die Standardfehler der Methode B der Datensätze Leo7 und Leo8 sind für $\mathrm{D}^{\mathrm{b}}$ größer als für $\mathrm{D}^{\mathrm{d}}$, teilweise ist die Differenz erheblich (s. Tab. 6.1 und 6.2, insbesondere die mittlere und höchste Auflösungsstufe in Tab. 6.1, Methode B). Dieses Phänomen tritt auf, wenn der untere Cutoff im Vergleich zur Auflösung hoch angesetzt wurde (dann tritt beim Boxcounting Verfahren die bereits erwähnte „Treppenstufenfunktion“ auf) und gleichzeitig relativ wenige Datenpaare in die lineare Regression eingingen. Bei Leo9 ist die Situation nicht so eindeutig, hier sind die Standardfehler von $\mathrm{D}^{\mathrm{d}}$ ungefähr gleich groß wie für $\mathrm{D}^{\mathrm{b}}$ (s. Tab. 6.3). Als Ursache ist die bei der Dilatation verwendete doppelte Schrittweite zu sehen, die bei einer im Gegensatz zum Boxcountingverfahren geringeren Anzahl von Datenpaaren zu einem höheren Standardfehler führt.

\subsubsection{Abweichung der Steigung D von der Linearität}

Die in allen Messserien zu beobachtende Zunahme der Werte für D reicht von $D^{d}=1,564$ bis $\mathrm{D}^{\mathrm{b}}=1,915$ für Leo7 (Tab. 6.1), von $\mathrm{D}^{\mathrm{d}}=1,580$ bis $\mathrm{D}^{\mathrm{b}}=1,914$ für Leo8 (Tab. 6.2) und von $D^{d}=1,611$ bis $D^{b}=1,935$ für Leo9 (Tab. 6.3). Die größte Veränderung liegt für Leo7 und Leo8 im Bereich der mittleren Vergrößerungsstufe, für Leo9 zwischen der ersten und der zweiten und der dritten und vierten Vergrößerungsstufe und für alle im Größenbereich zwischen 1 und $20 \mu \mathrm{m}$. Bei der Betrachtung der Richardson Plots von Einzelbildern der mittleren Vergrößerungsstufe fällt eine systematische Krümmung der Messkurve auf, besonders bei den Bildern, deren Regressionskoeffizient $\left(\mathrm{R}^{2}\right)$ relativ schlecht war (s. Abb. 6.3). Zum Vergleich, die $\mathrm{R}^{2}$ für jede Vergrößerungsstufe in Abb. 5.6 sind alle besser und besonders für die beiden niedrigsten Auflösungsstufen ist die Linearität mit $\mathrm{R}^{2}>0,999$ stark ausgeprägt (s. auch die Abb. 6.1 und 6.2). 
Die Wahl der Schrittweite (linear oder exponentiell) führt bei einzelnen Vergrößerungsstufen zu dem Ergebnis, dass bei der Berechnung von D durch lineare Regression die fraktale Dimension durch die Häufung der Daten im Bereich großer Skalierungselemente übergewichtet wird (s. Abb. 6.3). Der asymptotische Verlauf bleibt jedoch erhalten, was sich auch an den Residuen zeigt.
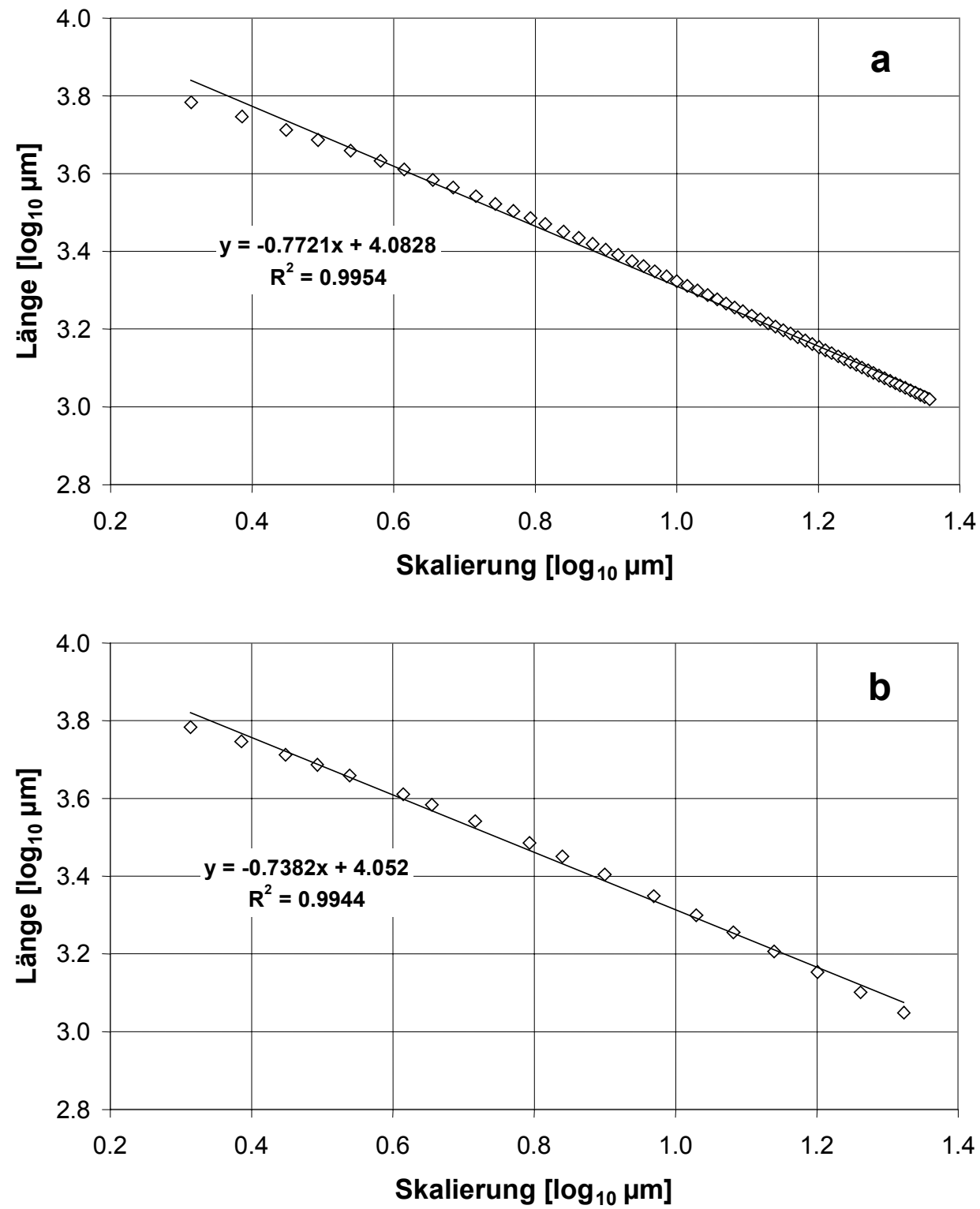

Abb. 6.3: Richardson-Plot einer Aufnahme der Serie L8D-1500, der mittleren Vergrößerungsstufe mit einer besonders ausgeprägten Abweichung der Messpunkte von der Regressionsgeraden. (a) Der lineare Abstand der Schrittweiten führt bei logarithmischer Auftragung zu einer Häufung der Daten in Richtung großer Skalierungselemente. (b) Der gleiche Datensatz wie in (a) mit exponentieller Schrittweite.

Die zu den in Abb. 6.3. gezeigten Richardson-Plots gehörende REM-Aufnahme wird in Abb. 6.4 gezeigt. Die Grenzlinie zwischen Pore und Matrix ist schwarz markiert, so wie sie als Struktur untersucht wurde. Es sind deutlich verschiedene Porengrößenbereiche zu erkennen: Große Poren, die durch die Anordnung von Einzelkörnern der Schlufffraktion verursacht worden sind und kleinere, die von der feinen Matrix gebildet wurden. Die zu Einzelmessungen der mittleren Vergrößerungsstufe gehörenden Bilder mit den niedrigsten Korrelationskoeffizienten weisen alle diesen deutlichen Unterschied zwischen zwei 
Porenbereichen auf, die durch unterschiedliche Genese entstanden sind und unterschiedliche Muster zeigen. Bei dem Vergleich von Einzelbildern verschiedener Vergrößerungsstufen fällt auf, dass die mit der niedrigsten Stufe aufgenommenen Bilder viel unregelmäßiger erscheinen als die mit der höchsten Stufe aufgenommenen (s. Abb. 4.7).

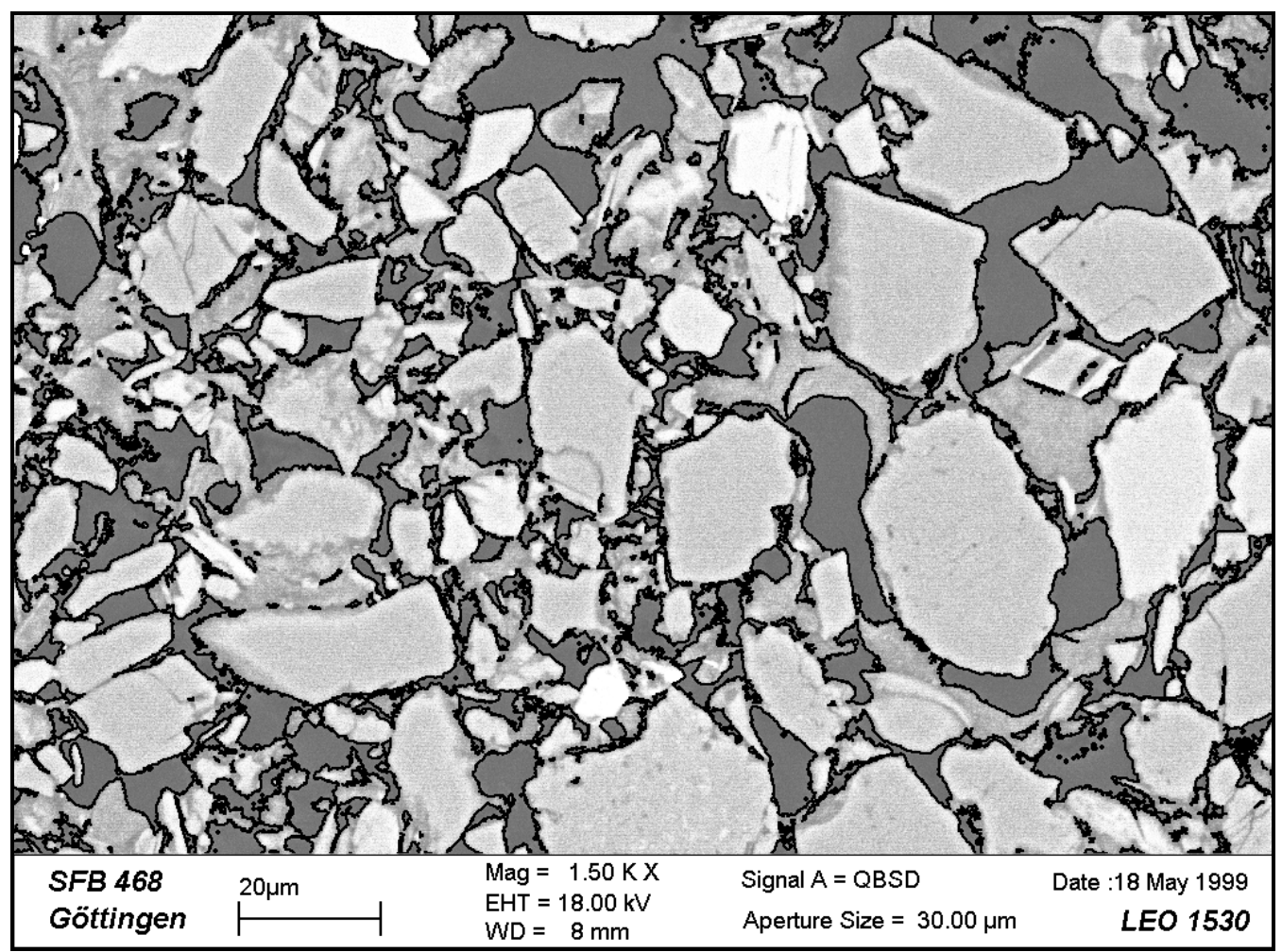

Abb. 6.4: REM-Aufnahme mit deutlich unterschiedlichen Porengrößen. Die Grenzlinie ist mit einem Pixel Breite schwarz markiert. Die Poren erscheinen dunkel und die Matrix hell.

So wird das Vorhandensein einer geringeren fraktalen Dimension bei höherer Auflösung als der Unterschied zwischen Struktur und Textur interpretiert. Dies unterstützt die These, dass im Grunde genommen zwei verschiedene Bereiche mit unterschiedlichen Mustern erfasst wurden: (i) Die Anordnung von einzelnen Schluffkörnern zueinander, die Struktur, und (ii) die Oberflächenrauhigkeit der einzelnen Körner, die Textur. Orford und Whalley (1983) beobachteten denselben Verlauf des Richardson Plots für einzelne Partikel, die großräumig unregelmäßig und im Kleinen regelmäßig geformt sind, und sprechen von struktureller und textureller Fraktalität.

\subsubsection{Anpassung von Regressionsgeraden an alle Vergrößerungsstufen}

Um den Größenbereich, in dem der Wechsel von einer Fraktalität zur anderen stattfindet, genauer lokalisieren zu können, wurden wie in Kapitel 5.1.5 beschrieben ein und zwei Regressionsgeraden angepasst. Der Schnittpunkt der zwei Geraden wird als Grenze zwischen dem durch die Textur festgelegten kleineren und dem durch die Struktur festgelegten größeren Skalenbereich definiert. Die Ergebnisse für Leo8, Dilatation, sind in den Abb. 6.5 und 6.6 dargestellt, für Boxcounting bereits in Kapitel 5 unter den Abb. 5.7 und 5.9. Die graphischen Darstellungen für Leo7 und Leo9 befinden sich im Anhang unter A.3 und A.4. Zur besseren Übersichtlichkeit werden hier die Ergebnisse tabellarisch 
dargestellt (Tab. 6.4 bis Tab. 6.6). Die Auflösung und der verwendete Datenbereich pro Vergrößerungsstufe entsprechen den in den Tabellen 6.1 bis 6.3 dargestellten Werten.

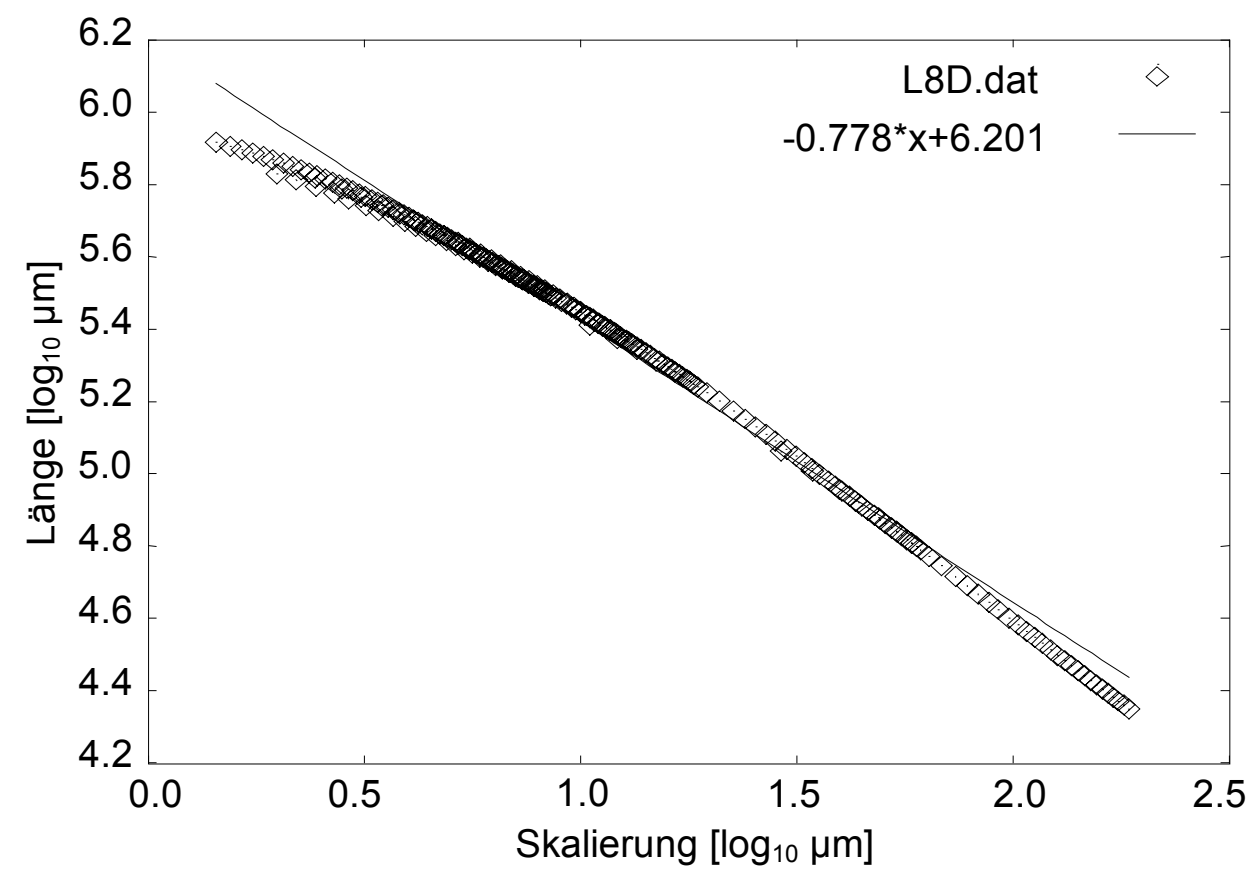

Abb. 6.5: Alle Vergrößerungsstufen des Schliffes Leo8, nach der Methode B gemittelte und bezüglich des y-Achsenabschnitts angepasste Messdaten der Dilatations-Methode. Die Regressionsgleichung ist angegeben.

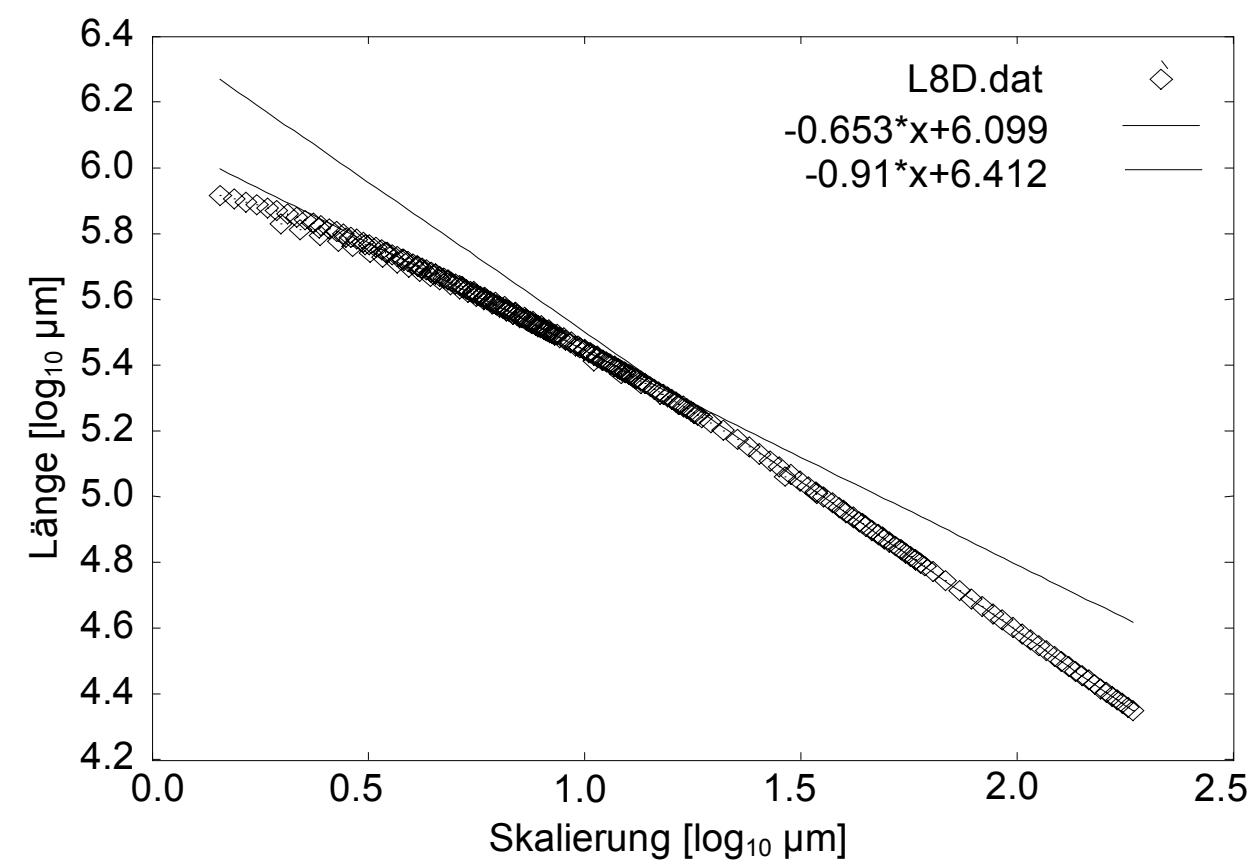

Abb. 6.6: Anpassung von zwei Geraden an die in Abb. 6.5 gezeigten Daten des Schliffes Leo8. Die Regressionsgleichungen sind angegeben.

Bei dem Dilatationsverfahren ist die Abweichung von einer Geraden viel ausgeprägter als beim Boxcounting-Verfahren. Der Grund ist in der messtechnisch bedingten Steigung zu sehen, die bei Dilatation um den Betrag von 1 geringer ist, was zu einer differenzierteren Darstellung der Messgröße auf der y-Achse führt (zum Vergleich s. Abb. 6.6 und 5.9). 
Tab. 6.4: Fraktale Dimensionen der Serie Leo7, vertikale Schliffausrichtung, gemessen mit Boxcounting $\left(D^{b}\right)$ und Dilatation $\left(D^{d}\right)$. Die Berechnung erfolgte über alle Vergrößerungsstufen für die gemittelten Rohdaten (Methode B). Die als Optimierungskriterium benutzten absoluten Abweichungen stehen in Klammern. $\mathrm{D}_{\mathrm{T}}$ ist die fraktale Dimension über den gesamten Vergrößerungsbereich, $\mathrm{D}_{1}$ wird der Textur im Bereich kleiner Skalierungselemente, $\mathrm{D}_{2}$ der Struktur im Bereich großer Skalierungselemente zugeordnet. Die hinter $D_{2}$ dargestellte absolute Abweichung bezieht sich auf $D_{1}$ und $D_{2}$. Eine lineare Schrittweite bedeutet, dass alle Daten berücksichtigt wurden. Eine exponentiell wachsende Schrittweite bedeutet, dass der Abstand der Skalierungsschritte exponentiell ansteigt.

\begin{tabular}{cccccc}
\hline $\begin{array}{c}\text { Skalierungs- } \\
\text { schrittweite }\end{array}$ & $\mathrm{D}_{\mathrm{T}}^{\mathrm{b}}$ & $\mathrm{D}_{1}^{\mathrm{b}}$ & $\mathrm{D}_{2}^{\mathrm{b}}$ & $\begin{array}{c}\text { Schnittpunkt } \\
{[\mu \mathrm{m}]}\end{array}$ \\
\hline linear & $1,798(0,037)$ & & & & - \\
\hline exp. & $1,812(0.038)$ & & & & - \\
\hline linear & & 1,575 & 1,884 & $(0,024)$ & 11,310 \\
\hline exp. & 1,572 & 1,897 & $(0,020)$ & 12,642 \\
\hline $\begin{array}{c}\text { Skalierungs- } \\
\text { schrittweite }\end{array}$ & $\mathrm{D}_{\mathrm{T}}^{\mathrm{d}}$ & $\mathrm{D}_{1}^{\mathrm{d}}$ & & $\mathrm{D}_{2}^{\mathrm{d}}$ & $\begin{array}{c}\text { Schnittpunkt } \\
{[\mu \mathrm{m}]}\end{array}$ \\
\hline linear & $1,797(0,039)$ & & & & - \\
\hline exp. & $1,808(0,040)$ & & & & - \\
\hline linear & & 1,611 & 1,913 & $(0,011)$ & 14,979 \\
\hline exp. & & 1,583 & 1,908 & $(0,013)$ & 13,218 \\
\hline
\end{tabular}

$\mathrm{Ob}$ die Skalierungsschrittweiten linear oder exponentiell gewählt wurden macht sich für eine fraktale Dimension über den gesamten Vergrößerungsbereich $\left(\mathrm{D}_{\mathrm{T}}\right)$ bei Leo7 und Leo8 wenig bemerkbar. Unterschiede ergeben sich vor allem für Leo9. Der Grund für die im Vergleich zu Messungen an einer Vergrößerungsstufe (s. Abb. 6.3) relativ geringen Unterschiede ist darin $\mathrm{zu}$ sehen, dass für die Daten, die von verschiedenen Vergrößerungsstufen angepasst wurden, die Häufung von Datenpaaren in Richtung großer Skalierungselemente vier- bzw. fünfmal vorkommt. Die absolute Abweichung ist für die exponentiell verteilten Messschritte bei allen Messserien etwas höher. Die Abbildungen für die Anpassung von zwei Geraden an die Messergebnisse für exponentielle Schrittweiten finden sich im Anhang unter Abb. A.5, A.6 und A.7 für die Serien Leo7, Leo8 und Leo9.

Bei der Anpassung von zwei Geraden wirkt sich die Wahl der Schrittweiten vor allem bei der Dilatation auf den Schnittpunkt der Regressionsgeraden und damit auf $\mathrm{D}_{1}^{\mathrm{d}}$ und $\mathrm{D}_{2}^{\mathrm{d}}$ aus. Dies ist auf die Häufung von Datenpunkten durch die Anpassung verschiedener Vergrößerungsstufen zurückzuführen, wenn sich die Datenbereiche innerhalb der Cutoffs überschneiden und das Minimum der absoluten Abweichung, das als Optimierungskriterium benutzt wird, nicht deutlich ausgeprägt ist (s. Tab. 6.1 bis 6.3 und Abb. 6.5 und 6.6).

Ein Vergleich der Messverfahren zeigt nur für Leo9 $\mathrm{D}^{\mathrm{b}}>\mathrm{D}^{\mathrm{d}}$ (s. Tab. 6.6). Bei Leo7 ist bis auf $\mathrm{D}_{\mathrm{T}}$ immer $\mathrm{D}^{\mathrm{b}}<\mathrm{D}^{\mathrm{d}}$ (s. Tab. 6.4) und bei Leo8 bis auf die Ausnahme von $\mathrm{D}_{1}$, exponentielle Schrittweiten, $\mathrm{D}^{\mathrm{b}}<\mathrm{D}^{\mathrm{d}}$ (s. Tab. 6.5). Die für Leo7 und Leo8 gemessenen $\mathrm{D}_{\mathrm{T}}$ weisen in Bezug auf die Messverfahren Boxcounting oder Dilatation eine geringe Differenz auf $(\Delta \leq 0,005)$ während die $\mathrm{D}_{\mathrm{T}}$ von Leo9 um maximal $\Delta=0,037$ differieren. Die 
Spanne der $\mathrm{D}_{\mathrm{T}}$ reicht für exponentielle Schrittweiten von $\mathrm{D}^{\mathrm{d}}=1,716$ für Leo9 bis $\mathrm{D}^{\mathrm{b}}=1,812$ für Leo7.

Tab. 6.5: Fraktale Dimensionen der Serie Leo8, horizontale Schliffausrichtung, gemessen mit Boxcounting $\left(D^{b}\right)$ und Dilatation $\left(D^{d}\right)$.

\begin{tabular}{|c|c|c|c|c|}
\hline $\begin{array}{l}\text { Skalierungs- } \\
\text { schrittweite }\end{array}$ & $\mathrm{D}_{\mathrm{T}}^{\mathrm{b}}$ & $\mathrm{D}_{1}^{\mathrm{b}}$ & $\mathrm{D}_{2}^{\mathrm{b}}$ & $\begin{array}{c}\text { Schnittpunkt } \\
{[\mu \mathrm{m}]}\end{array}$ \\
\hline linear & $1,747(0,037)$ & & & - \\
\hline exp. & $1,746(0,038)$ & & & - \\
\hline linear & & 1,589 & $1,880 \quad(0,031)$ & 14,022 \\
\hline exp. & & 1,601 & $1,886 \quad(0,022)$ & 14,579 \\
\hline $\begin{array}{l}\text { Skalierungs- } \\
\text { schrittweite }\end{array}$ & $\mathrm{D}_{\mathrm{T}}^{\mathrm{d}}$ & $\mathrm{D}_{1}^{\mathrm{d}}$ & $\mathrm{D}_{2}^{\mathrm{d}}$ & $\begin{array}{c}\text { Schnittpunkt } \\
{[\mu \mathrm{m}]}\end{array}$ \\
\hline linear & $1,778(0,030)$ & & & - \\
\hline exp. & $1,783(0,038)$ & & & - \\
\hline linear & & 1,653 & $1,910 \quad(0,013)$ & 16,559 \\
\hline exp. & & 1,591 & $1,894 \quad(0,017)$ & 12,350 \\
\hline
\end{tabular}

Tab. 6.6: Fraktale Dimensionen der Serie Leo9, horizontale Schliffausrichtung, gemessen mit Boxcounting $\left(D^{b}\right)$ und Dilatation. $\left(D^{d}\right)$.

\begin{tabular}{|c|c|c|c|c|}
\hline $\begin{array}{l}\text { Skalierungs- } \\
\text { schrittweite }\end{array}$ & $\mathrm{D}_{\mathrm{T}}^{\mathrm{b}}$ & $\mathrm{D}_{1}^{\mathrm{b}}$ & $\mathrm{D}_{2}^{\mathrm{b}}$ & $\begin{array}{c}\text { Schnittpunkt } \\
{[\mu \mathrm{m}]}\end{array}$ \\
\hline linear & $1,774(0,030)$ & & & - \\
\hline exp. & $1,754(0,034)$ & & & - \\
\hline linear & & 1,620 & $1,862 \quad(0,019)$ & 5,692 \\
\hline exp. & & 1,624 & $1,865 \quad(0,019)$ & 5,842 \\
\hline $\begin{array}{l}\text { Skalierungs- } \\
\text { schrittweite }\end{array}$ & $\mathrm{D}_{\mathrm{T}}^{\mathrm{d}}$ & $\mathrm{D}_{1}^{\mathrm{d}}$ & $\mathrm{D}_{2}^{\mathrm{d}}$ & $\begin{array}{c}\text { Schnittpunkt } \\
{[\mu \mathrm{m}]}\end{array}$ \\
\hline linear & $1,733(0,028)$ & & & - \\
\hline exp. & $1,716(0,030)$ & & & - \\
\hline linear & & 1,614 & $1,841 \quad(0,015)$ & 6,430 \\
\hline exp. & & 1,604 & $1,829 \quad(0,018)$ & 5,855 \\
\hline
\end{tabular}

Die der Textur zugeordnete kleinere fraktale Dimension reicht von $\mathrm{D}_{1}=1,572$ (für Leo7, Boxcounting und exponentielle Schrittweiten, s. Tab. 6.4) bis $\mathrm{D}_{1}=1,653$ (für Leo8, Dilatation, lineare Schrittweiten s. Tab. 6.5). Die größere fraktale Dimension, die der Struktur zugeordnet wird, erstreckt sich von $\mathrm{D}_{2}=1,829$ für Leo9, Dilatation und exponentielle Schrittweiten (s. Tab. 6.6) bis $\mathrm{D}_{2}=1,913$ für Leo7, Dilatation und exponentielle Schrittweiten.

Werden Leo7 (vertikal) und Leo8 (horizontal), die aus dem gleichen Kubiena-Klotz stammen, miteinander verglichen, ergibt sich für $\mathrm{D}_{\mathrm{T}}$ immer ein höherer Wert für Leo7. Für 
Boxcounting betragen die Differenzen $\Delta=0,051$ (lineare Schrittweiten) und $\Delta=0,066$ (exponentielle Schrittweiten). Bei der Dilatation beträgt die Differenz $\Delta=0,019$ (lineare Schrittweiten) und $\Delta=0,025$ (exponentielle Schrittweiten). $\mathrm{D}_{1}$ und $\mathrm{D}_{2}$ der beiden Schliffe direkt zu vergleichen ist nicht sinnvoll, da der als optimal bestimmte Schnittpunkt der Geraden für jeden Schliff und jedes Messverfahren bei einer anderen Größe des skalierenden Elementes liegt. Wie bereits erwähnt, wächst die fraktale Dimension kontinuierlich mit der Größe des für die Messung verwendeten skalierenden Elements. Da der Größenbereich der Messungen an beiden Schliffen nicht übereinstimmt (für Leo7 reicht er von 1,72 bis $221,95 \mu \mathrm{m}$ und für Leo8 von 1,38 bis $185,60 \mu \mathrm{m}$ ), kann die beobachtete Differenz nicht auf die unterschiedlichen Schliffrichtungen zurückgeführt werden.

Der Schnittpunkt beider Geraden liegt für Leo9 mit 5,855 bis 6,430 $\mu \mathrm{m}$ (s. Tab. 6.6) deutlich niedriger als für Leo7 mit 11,310 bis $14,979 \mu \mathrm{m}$ (s. Tab. 6.4) und für Leo8 mit 12,350 bis $16,559 \mu \mathrm{m}$ (s. Tab. 6.6). Der kleinste Wert für den Schnittpunkt wurde für Leo7 und Leo9 für Boxcounting und lineare Schrittweiten ermittelt, für Leo8 für Dilatation und exponentielle Schrittweiten. Der jeweils größte Wert für den Schnittpunkt findet sich für alle Messserien bei Dilatation und linearen Schrittweiten, hier ist auch die Differenz zu den für exponentielle Schrittweiten ermittelten Schnittpunkten am größten. Ob die deutlich „kleineren“ Schnittpunkte von Leo9 auf die im Bereich einer kleineren Skalierung durchgeführten Messungen (0,44 bis 98,26 $\mu \mathrm{m}$, s. Tab. 6.1) oder auf die doppelt so hohe Auflösung zurückzuführen sind, lässt sich anhand der bis hier aufgeführten Auswertungen nicht beurteilen.

\subsubsection{Messungen an lichtmikroskopischen Aufnahmen}

Die hier dargestellten Ergebnisse beziehen sich zunächst auf die für die Serien Leo7, Leo8 und Leo9 untersuchten Schliffe, die bei den verwendeten Einstellungen im Lichtmikroskop fast in ihrer vollständigen Größe abgebildet werden (s. Abb. 4.5, A.1 und A.2). Die Ergebnisse sind nicht direkt mit den aus REM-Aufnahmen erzielten vergleichbar. Auffällig ist vor allem, dass im Größenbereich von kleinen Skalierungselementen (bei einer Bildauflösung von rund $8 \mu \mathrm{m} /$ Pixel) die fraktale Dimension gegen 1 geht, was der euklidischen Dimension einer Länge entspricht (s. Abb. 6.7 und 6.8). Erst ab einer Größe des skalierenden Elementes von rund $500 \mu \mathrm{m}$ kann eine fraktale Dimension gemessen werden. Der Grund ist in der Schichtdicke des Dünnschliffs von ca. $30 \mu \mathrm{m}$ zu sehen, die bei mikroskopischen Aufnahmen im Durchlicht in ihrer Gesamtheit durchstrahlt wird. Im Gegensatz dazu beträgt die Eindringtiefe der Elektronen bei den REM-Aufnahmen ca. $1 \mu \mathrm{m}$. So werden bei den Aufnahmen mit dem Wild-Photomakroskop einzelne Poren mit geglätteten Rändern abgebildet und nicht das komplexe Netzwerk wie in den REMBildern. Das erklärt, weshalb $\mathrm{D}$ für die erste Gerade im Bereich kleiner Skalierungselemente nahe 1 bzw. sogar $<1$ liegt (s. die Geradengleichungen in den Abb. 6.7 und 6.8 und die Werte für $\mathrm{D}_{1}$ in den Tabellen 6.7 und 6.9). Gemessen wird die Rauhigkeit der Grenzlinie einzelner Poren. 


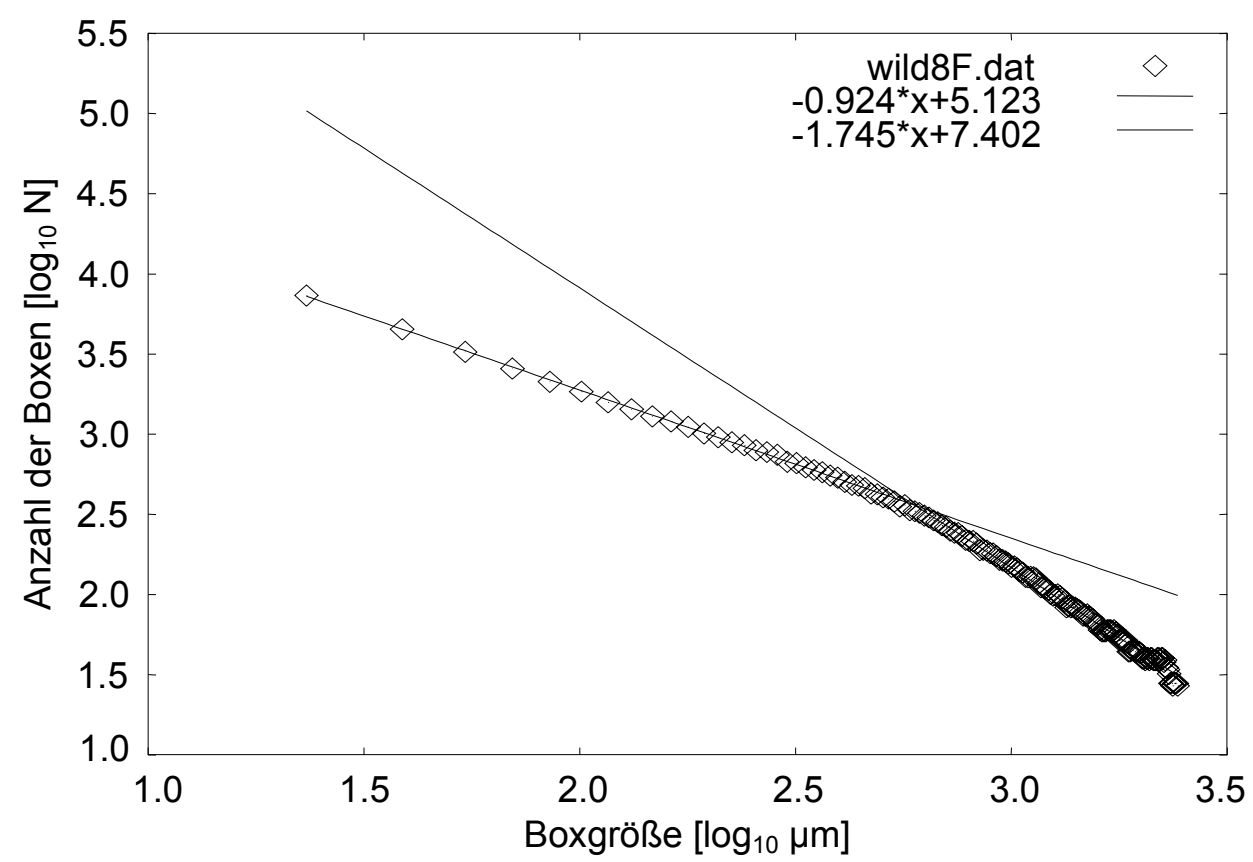

Abb. 6.7: Anpassung von zwei Geraden an die mit dem Boxcounting-Verfahren gewonnenen Daten für das mit dem Wild-Photomakroskop aufgenommene Einzelbild des für die Serie Leo8 verwendeten Dünnschliffs.

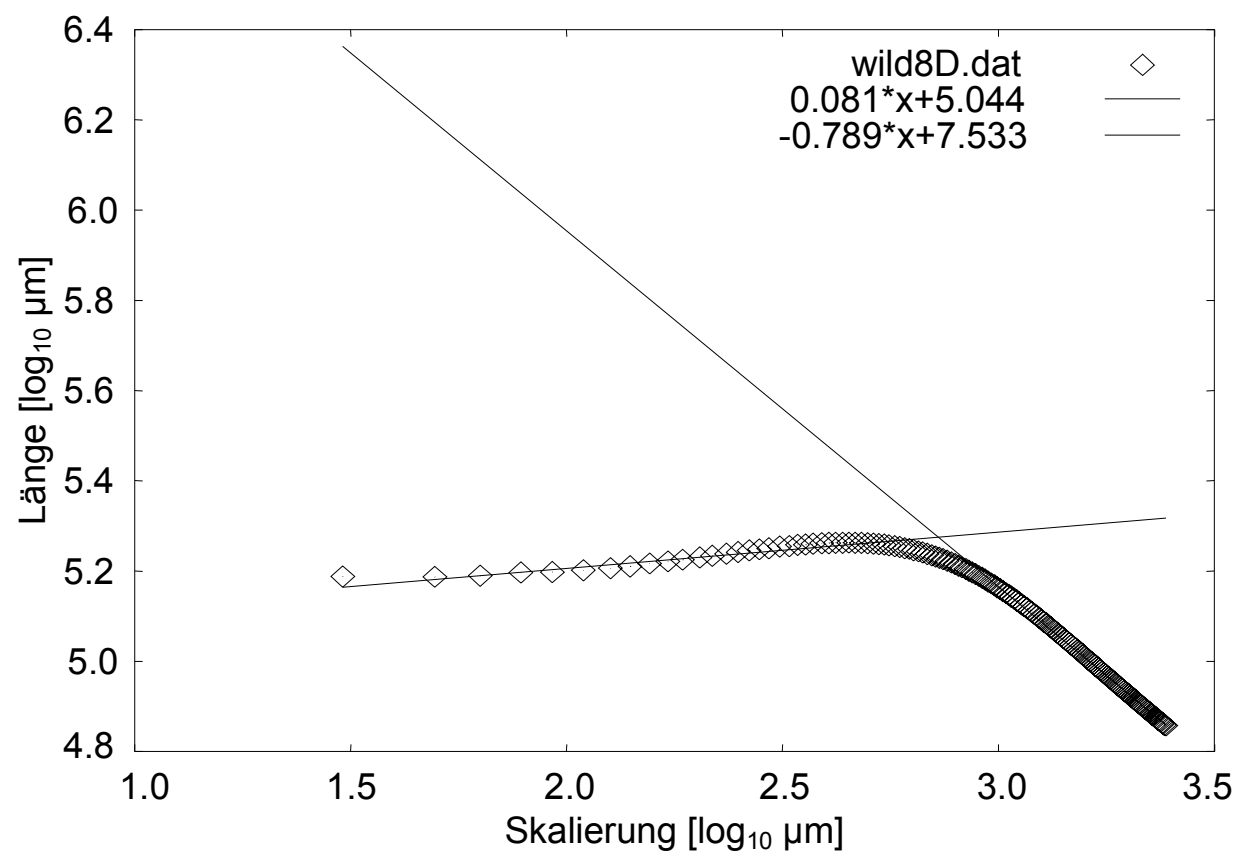

Abb. 6.8: Die gleiche Aufnahme wie für Abb. 6.7, jedoch mit Dilatation gemessen.

Beim Dilatationsverfahren nimmt die Länge der Umhüllenden zunächst zu und nicht, wie nach der Definition von selbstähnlichen Objekten zu erwarten, ab. Das liegt daran, dass die Länge der Umhüllenden aus dem Quotienten von Fläche und Durchmesser des Strukturelementes bestimmt wird, und für einzelne isolierte Poren diese Fläche in das Poreninnere hinein nicht unbegrenzt wachsen kann. Erst wenn die Skalierungselemente die Größenordnung der Anordnung der einzelnen Poren zueinander erreichen, nimmt die Länge der Umhüllenden mit wachsendem Skalierungselement ab, und aus den jeweils logarithmisch aufgetragenen Werten kann die fraktale Dimension abgelesen werden. Beim 
Boxcounting-Verfahren tritt ein ähnlicher Effekt auf, wenn die Boxen die Grenzlinie einzelner Poren bedecken und bei ihrer Vergrößerung die Anordnung der Poren zueinander erfassen.

Bei der Berechnung von $\mathrm{D}_{1}$ aus der Geradengleichung des Dilatationsverfahrens muss beachtet werden, dass laut Gl. 5.2 die Steigung aus dem Summanden 1- $\mathrm{D}_{\mathrm{L}}^{\mathrm{d}}$ besteht. Wird im Bereich kleiner Skalierungselemente eine positive Steigung beobachtet führt dies zu einer Längendimension $\mathrm{D}<1$ (s. Abb. 6.8 und Tab. 6.4).

\subsubsection{Untersuchung der für die REM-Aufnahmen verwendeten Dünnschliffe}

In der Tabelle 6.7 sind die Ergebnisse für eine Anpassung von zwei Geraden (s. Kapitel 5.1.5) für die drei lichtmikroskopischen Bilder Wild7, 8 und 9 dargestellt.

Tab. 6.7: Fraktale Dimensionen der für die Leo-Serien 7, 8 und 9 benutzten Schliffe, gemessen an mit dem Wild-Photomakroskop aufgenommenen Einzelbildern mit Boxcounting $\left(D^{\mathrm{b}}\right)$ und Dilatation $\left(\mathrm{D}^{\mathrm{d}}\right.$. Für Wild7 ist die Schliffausrichtung vertikal, für Wild8 und 9 horizontal. $\mathrm{D}_{\text {I }}$ ist die fraktale Dimension im Größenbereich einzelner Poren bzw. ihrer Grenzlinien und $\mathrm{D}_{\mathrm{II}}$ ist die fraktale Dimension der Anordnung der Einzelporen zueinander. Die als Optimierungskriterium benutzte absolute Abweichung ist in Klammern dargestellt und bezieht sich auf $D_{\mid}$und $D_{\|}$. Eine lineare Schrittweite bedeutet, dass alle Daten berücksichtigt wurden. Eine exponentiell wachsende Schrittweite bedeutet, dass der Abstand der Skalierungsschritte exponentiell ansteigt. Der Skalierungsbereich erstreckt sich von 23,29 bis $2437,82 \mu \mathrm{m}$.

\begin{tabular}{|c|c|c|c|c|}
\hline Schliff & $\begin{array}{l}\text { Skalierungs- } \\
\text { schrittweite }\end{array}$ & $\mathrm{D}_{\mathrm{I}}^{\mathrm{b}}$ & $\mathrm{D}_{\mathrm{II}}^{\mathrm{b}}$ & $\begin{array}{c}\text { Schnittpunkt } \\
{[\mu \mathrm{m}]}\end{array}$ \\
\hline \multirow{2}{*}{ Wild7 } & linear & 0,922 & $1,791 \quad(0,023)$ & 596,33 \\
\hline & exp. & 0,921 & $1,783 \quad(0,022)$ & 590,85 \\
\hline \multirow{2}{*}{ Wild8 } & linear & 0,924 & $1,745 \quad(0,026)$ & 596,51 \\
\hline & exp. & 0,919 & $1,699 \quad(0,024)$ & 567,17 \\
\hline \multirow{2}{*}{ Wild9 } & linear & 0,995 & $1,642 \quad(0,034)$ & 329,71 \\
\hline & exp. & 1,046 & $1,626 \quad(0,033)$ & 362,19 \\
\hline Schliff & $\begin{array}{l}\text { Skalierungs- } \\
\text { schrittweite }\end{array}$ & $\mathrm{D}_{\mathrm{I}}^{\mathrm{d}}$ & $\mathrm{D}_{\mathrm{II}}^{\mathrm{d}}$ & $\begin{array}{l}\text { Schnittpunkt } \\
{[\mu \mathrm{m}]}\end{array}$ \\
\hline \multirow{2}{*}{ Wild7 } & linear & 0,910 & $1,808 \quad(0,014)$ & 693,13 \\
\hline & exp. & 0,918 & $1,796 \quad(0,016)$ & 693,66 \\
\hline \multirow{2}{*}{ Wild8 } & linear & 0,919 & $1,789 \quad(0,014)$ & 725,33 \\
\hline & exp. & 0,918 & $1,765 \quad(0,016)$ & 707,88 \\
\hline \multirow{2}{*}{ Wild9 } & linear & 1,004 & $1,647 \quad(0,025)$ & 404,79 \\
\hline & exp. & 1,004 & $1,528 \quad(0,028)$ & 326,94 \\
\hline
\end{tabular}

Im Gegensatz zu der an den rasterelektronischen Bildern beobachteten strukturellen und texturellen Fraktalität stellt hier $\mathrm{D}_{\mathrm{I}}$ die Dimension der Umrisslinien einzelner Poren dar und $\mathrm{D}_{\mathrm{II}}$ die Anordnung der Einzelporen zueinander. Der Schnittpunkt beider Geraden zeigt die Größenordnung des skalierenden Elementes an, ab der diese Anordnung in der Fläche erfasst wird (s. Abb. 6.7 und 6.8 sowie A.8 und A.9). 
Im Größenbereich der Anordnung der Poren zueinander ist mit Ausnahme von Wild9 bei exponentiellen Schrittweiten $D_{\mathrm{II}}^{\mathrm{d}}>\mathrm{D}_{\mathrm{II}}^{\mathrm{b}}$. Die Differenz ist für Wild8 am größten. Hier macht sich die programmbedingte Wahl des Schnittpunkts bemerkbar. Er liegt für das Dilatationsverfahren im Bereich größerer Skalierungselemente als für Boxcounting, bei Wild8 ist diese Differenz mit $\Delta \approx 129 \mu \mathrm{m}$ besonders hoch. Der Übergangsbereich zwischen den beiden linearen Bereichen im doppellogarithmischen Plot ist bei der Dilatation besonders ausgeprägt (s. Abb. 6.8 sowie A.8 b und A.9 b). Um die Daten in diesem Bereich erfassen zu können, erreicht die zweite Gerade nicht die maximale Steigung, die sie für die größten Skalierungselemente aufweisen müsste.

Deutlich wird dieser Sachverhalt für die in Tabelle 6.8 dargestellten Ergebnisse. Es ist jeweils die fraktale Dimension aus dem linearen Teil des Richardson-Plots innerhalb des unteren und oberen Cutoffs angegeben (s. Kapitel 5.1.1). Der untere Cutoff wurde für jedes Bild einzeln bestimmt, wobei für Boxcounting das in Kapitel 5.1.1.1 beschriebene Verfahren eingesetzt wurde. Für Dilatation wurde der Bereich, ab dem der Korrelationskoeffizient $<-0,999$ wurde, als linear eingestuft. Die oberen Cutoffs waren programmbedingt vorgegeben und lagen bei rund 1/3 der Breite der schmalen Bildseite. Da mit Dilatation im Bereich größerer Skalierungselemente genauer gemessen werden kann als mit Boxcounting, liegt der obere Cutoff für Dilatation etwas höher. Die auf diese Weise ermittelten D stimmen für beide Messverfahren gut überein, obwohl der untere Cutoff für Dilatation (vor allem für Wild8 und Wild9) in einem Bereich von wesentlich größeren Skalierungselementen als für Boxcounting liegt. Die Übereinstimmung mit den in Tab. 6.7 dargestellten $\mathrm{D}_{\mathrm{II}}^{\mathrm{b}}$ und $\mathrm{D}_{\mathrm{II}}^{\mathrm{d}}$ ist für die Bilder Wild7 und Wild8 ebenfalls gut. Bei Wild9 ist dies nicht der Fall, hier wird mit der Anpassung einer Geraden im optimalen Bereich eine um rund 0,1 höhere fraktale Dimension ermittelt als durch die Anpassung von zwei Geraden. Die Differenz zwischen den für Wild9 angegebenen Schnittpunkten (Tab. 6.7) und unteren Cutoffs (Tab. 6.8) ist vor allem für Dilatation erheblich $(\Delta \approx 449 \mu \mathrm{m})$ und drückt sich in der in Richtung größerer Skalierungselemente höheren fraktalen Dimension aus.

Interessant ist der Vergleich zwischen den fraktalen Dimensionen der einzelnen Schliffe im makroskopischen Bereich, also $\mathrm{D}_{\mathrm{II}}$ in Tab. 6.7 und $\mathrm{D}$ in Tab. 6.8, mit den in den Tab. 6.4 bis 6.6 dargestellten $\mathrm{D}_{\mathrm{T}}$ der mit dem REM im mikroskopischen und submikroskopischen Bereich aufgenommenen Bilder. Für Wild7 und Wild8 stimmen die Ergebnisse gut überein (z. B. $\mathrm{D}_{\mathrm{T}}^{\mathrm{d}}=1,797$ für Leo7, lineare Schrittweiten (Tab. 6.4) und $\mathrm{D}=1,796$ für Wild7, Dilatation (Tab. 6.8) sowie $\mathrm{D}_{\mathrm{T}}^{\mathrm{b}}=1,747$ für Leo8 und lineare Schrittweiten (Tab. 6.5) und $\mathrm{D}_{\mathrm{II}}^{\mathrm{b}}=1,745$ für Wild8 (Tab. 6.7)). Bei Wild9 ist die Übereinstimmung der $\mathrm{D}_{2}$ beider Messverfahren in Tab. 6.7 mit den $\mathrm{D}_{\mathrm{T}}$ in Tab. 6.6 mit $0,086 \leq \Delta \leq 0,149$ nicht so gut. Beim Vergleich der gleichen $\mathrm{D}_{2}$ mit den in Tab. 6.8 fallen die Differenzen mit $-0,046 \leq \Delta \leq 0,033$ deutlich niedriger aus. Erstaunlich ist, dass die Ergebnisse aus unterschiedlichen bildgebenden Verfahren gewonnen wurden, deren Skalierungsbereiche nicht übereinstimmen. Während der den REM-Aufnahmen zugrunde liegende Skalierungsbereich für $\mathrm{D}_{\mathrm{T}}$ von Leo7 und Leo8 von 0,05 bis rund $200 \mu \mathrm{m}$ reicht, liegt der für $\mathrm{D}_{\mathrm{II}}$ bzw. D der lichtmikroskopischen Aufnahmen zwischen rund 600 und 2500 $\mu \mathrm{m}$. 
Tab. 6.8: Fraktale Dimensionen und für deren Berechnung relevante Parameter der für die Leo-Serien 7, 8 und 9 benutzten Schliffe, gemessen an mit dem Wild-Photomakroskop aufgenommenen Einzelbildern. $\mathrm{n}$ (B) ist die Anzahl der Datenpaare und se der Standardfehler des Regressionskoeffizienten für die Steigung. D wurde für jedes Bild und jede Methode aus dem optimalen linearen Bereich des Richardson-Plots bestimmt.

\begin{tabular}{|c|c|c|c|c|c|c|}
\hline Schliff & Methode & $\begin{array}{l}\text { Auflösung } \\
\text { [ } \mu \mathrm{m} / \text { Pixel] }\end{array}$ & $\begin{array}{c}\text { unterer } \\
\text { Cutoff }[\mu \mathrm{m}]\end{array}$ & $\begin{array}{c}\text { oberer } \\
\text { Cutoff }[\mu \mathrm{m}]\end{array}$ & $\mathrm{n}(\mathrm{B})$ & D mit se \\
\hline \multirow{2}{*}{ Wild7 } & Boxcounting & \multirow{2}{*}{7,763} & 613,28 & 2429,82 & 118 & $1,814(0,011)$ \\
\hline & Dilatation & & 761,43 & 2717,23 & 127 & $1,796(0,003)$ \\
\hline \multirow{2}{*}{ Wild8 } & Boxcounting & \multirow{2}{*}{7,763} & 566,70 & 2429,82 & 121 & $1,757(0,013)$ \\
\hline & Dilatation & & 839,10 & 2717,23 & 122 & $1,789(0,003)$ \\
\hline \multirow{2}{*}{ Wild9 } & Boxcounting & \multirow{2}{*}{7,763} & 489,07 & 2429,82 & 126 & $1,741(0,013)$ \\
\hline & Dilatation & & 853,78 & 2717,23 & 121 & $1,775(0,003)$ \\
\hline
\end{tabular}

Anhand der soweit dargestellten Ergebnisse kann kein Unterschied zwischen dem vertikal ausgerichteten Schliff Wild7 und den horizontal ausgerichteten Schliffen Wild8 und 9 festgestellt werden, was auf eine Isotropie der untersuchten Bodenstruktur hinweist. Im Folgenden soll daher die Bedeutung der Schliffrichtung auf die Fraktalität näher untersucht werden.

\subsubsection{Untersuchung von horizontal und vertikal ausgerichteten Dünnschliffen}

Die Serien WK2hor und WK2ver bestehen aus 7 bzw. 10 Bildern, die je einen einzelnen Schliff zeigen und einer Kubiena-Box entstammen. Leo7 ist Teil der Serie WK2ver und Leo8 ist Teil der Serie WK2hor. Die Dünnschliffe, die ebenfalls aus der für Leo9 verwendeten Box stammten, wurden nicht weiter untersucht. So liegt der Schwerpunkt der lichtmikroskopischen Untersuchungen auf den Serien WK2hor und WK2ver, um (i) den Größenbereich $>100 \mu \mathrm{m}$ zu erfassen und (ii) zu zeigen, wie die Struktur der für Leo7 und Leo8 verwendeten Dünnschliffe hinsichtlich eines größeren Stichprobenumfangs einzuordnen ist.

In den Abb. 6.9 und 6.10 sind die Ergebnisse beider Messverfahren für die vertikal ausgerichteten Schliffe dargestellt, in den Abb. 6.11 und 6.12 die für die horizontal ausgerichteten. Die Daten der Einzelbilder wurden arithmetisch gemittelt (Methode B, s. Abschnitt 5.1.2). Die Geradengleichungen sind jeweils angegeben und in der Tabelle 6.9 zusammengestellt. 


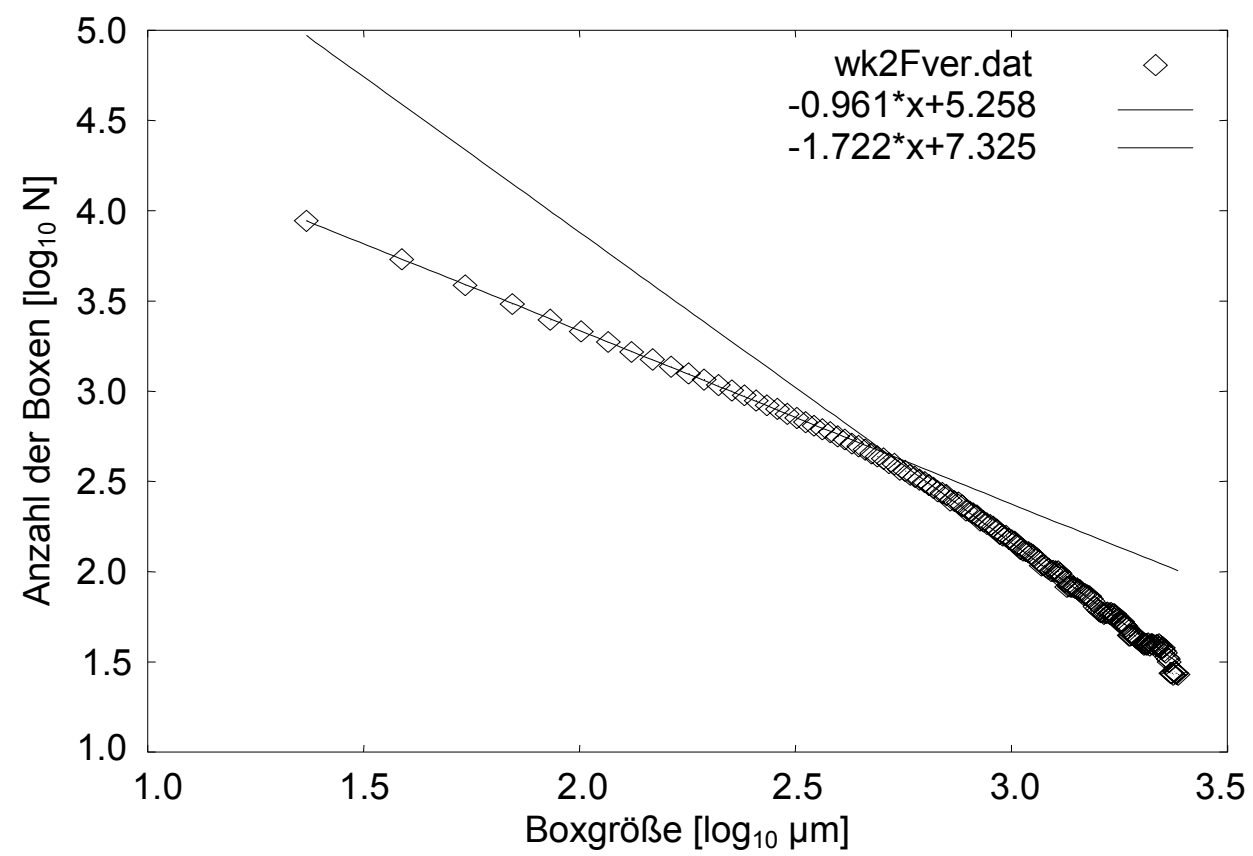

Abb. 6.9: Anpassung von zwei Geraden an die mit dem Boxcounting-Verfahren gewonnenen Daten (Mittelwerte von 10 Bildern) für die lichtmikroskopischen Aufnahmen der vertikal ausgerichteten Dünnschliffe einer Kubiena-Box.

Für Boxcounting und Dilatation sind die $\mathrm{D}_{1}<1$, was wie bei den Einzelbildern Wild7, 8 und 9 auf das bildgebende Verfahren zurückzuführen ist. In Tab. 6.9 ist $\mathrm{D}_{2}^{\mathrm{b}}<\mathrm{D}_{2}^{\mathrm{d}}$, wobei die Differenzen durch die jeweilige Wahl der Schnittpunkte beider Geraden beeinflusst werden. Hier wird deutlich, dass eine kontinuierliche Funktion, die die Abhängigkeit der fraktalen Dimension vom betrachteten Skalenbereich wiedergibt, eindeutigere Ergebnisse für D liefern sollte.

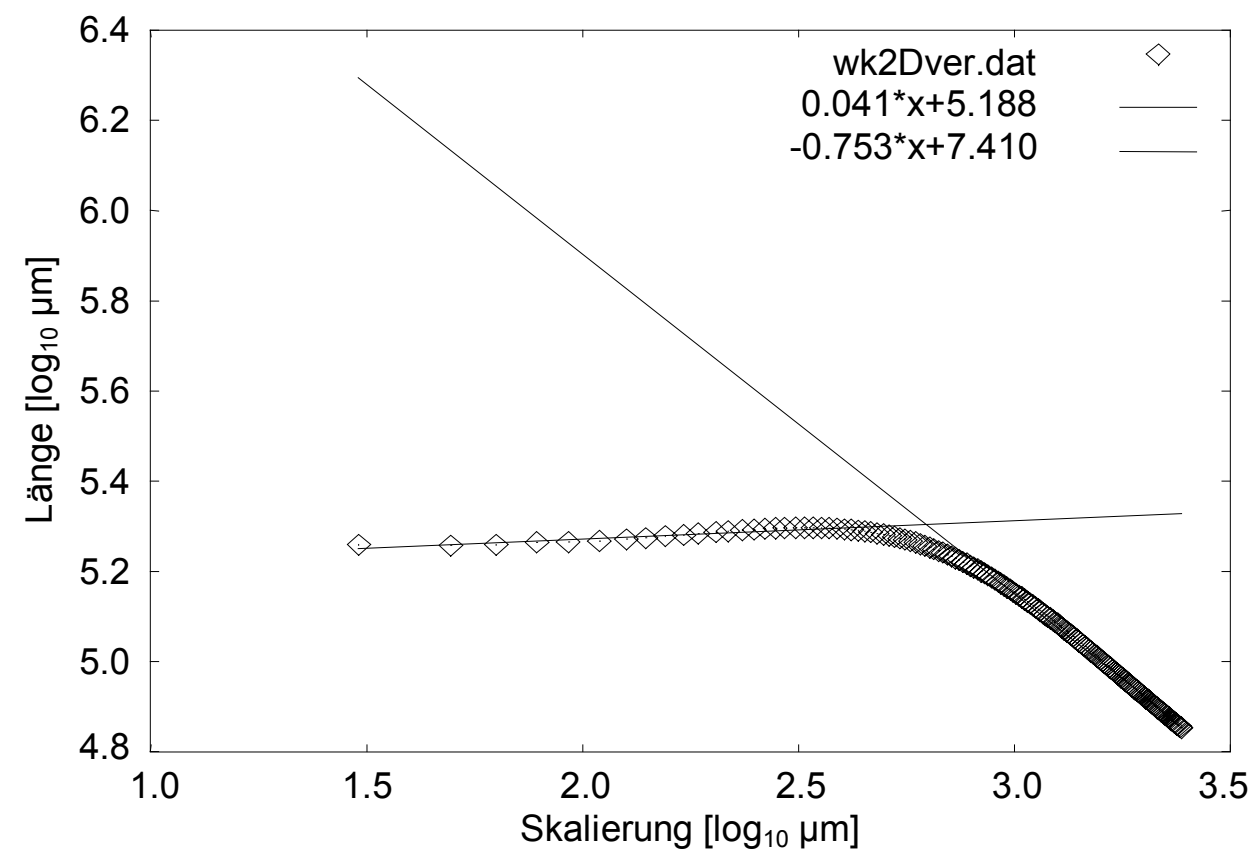

Abb. 6.10: Die gleichen Aufnahmen wie für Abb. 6.9, gemessen mit Dilatation. 
Die Ergebnisse für exponentiell wachsende Schrittweiten sind in Tab. 6.9 zusammengefasst.

Tab. 6.9: Fraktale Dimensionen der Serie Wild Klotz 2, gemessen mit Boxcounting $\left(D^{b}\right)$ und Dilatation $\left(D^{d}\right)$. Die Berechnung erfolgte differenziert in horizontale und vertikale Schliffausrichtungen aus den gemittelten Rohdaten (Methode B). $\mathrm{D}_{\mathrm{I}}$ ist die fraktale Dimension im Größenbereich einzelner Poren bzw. ihrer Grenzlinien und $\mathrm{D}_{\mathrm{II}}$ ist die fraktale Dimension der Anordnung der Einzelporen zueinander. Die als Optimierungskriterium benutzte absolute Abweichung ist in Klammern dargestellt und bezieht sich auf $D_{\mid}$und $D_{\| 1}$. Eine lineare Schrittweite bedeutet, dass alle Daten berücksichtigt wurden. Eine exponentiell wachsende Schrittweite bedeutet, dass der Abstand der Skalierungsschritte exponentiell ansteigt. Der Skalierungsbereich erstreckt sich von 23,29 bis $2437,82 \mu \mathrm{m}$.

\begin{tabular}{cccccc}
\hline \multirow{2}{*}{ Serie } & $\begin{array}{c}\text { Skalierungs- } \\
\text { schrittweite }\end{array}$ & $\mathrm{D}_{\mathrm{I}}^{\mathrm{b}}$ & \multicolumn{2}{c}{$\mathrm{D}_{\mathrm{II}}^{\mathrm{b}}$} & $\begin{array}{c}\text { Schnittpunkt } \\
{[\mu \mathrm{m}]}\end{array}$ \\
\hline \multirow{2}{*}{ WK2 ver } & linear & 0,961 & 1,722 & $(0,024)$ & 520,83 \\
\cline { 2 - 6 } & exp. & 0,961 & 1,680 & $(0,022)$ & 488,98 \\
\hline \multirow{2}{*}{ WK2 hor } & linear & 0,941 & 1,741 & $(0,026)$ & 428,64 \\
\cline { 2 - 6 } & exp. & 0,964 & 1,711 & $(0,025)$ & 429,22 \\
\hline \multirow{2}{*}{ Serie } & $\begin{array}{c}\text { Skalierungs- } \\
\text { schrittweite }\end{array}$ & $\mathrm{D}_{\mathrm{I}}^{\mathrm{d}}$ & & $\mathrm{D}_{\mathrm{II}}^{\mathrm{d}}$ & $\begin{array}{c}\text { Schnittpunkt } \\
{[\mu \mathrm{m}]}\end{array}$ \\
\hline \multirow{2}{*}{ WK2 ver } & linear & 0,959 & 1,753 & $(0,016)$ & 625,77 \\
\cline { 2 - 6 } & exp. & 0,959 & 1,715 & $(0,017)$ & 602,51 \\
\hline \multirow{2}{*}{ WK2 hor } & linear & 0,956 & 1,753 & $(0,018)$ & 514,40 \\
\cline { 2 - 6 } & exp. & 0,966 & 1,728 & $(0,021)$ & 511,88 \\
\hline
\end{tabular}

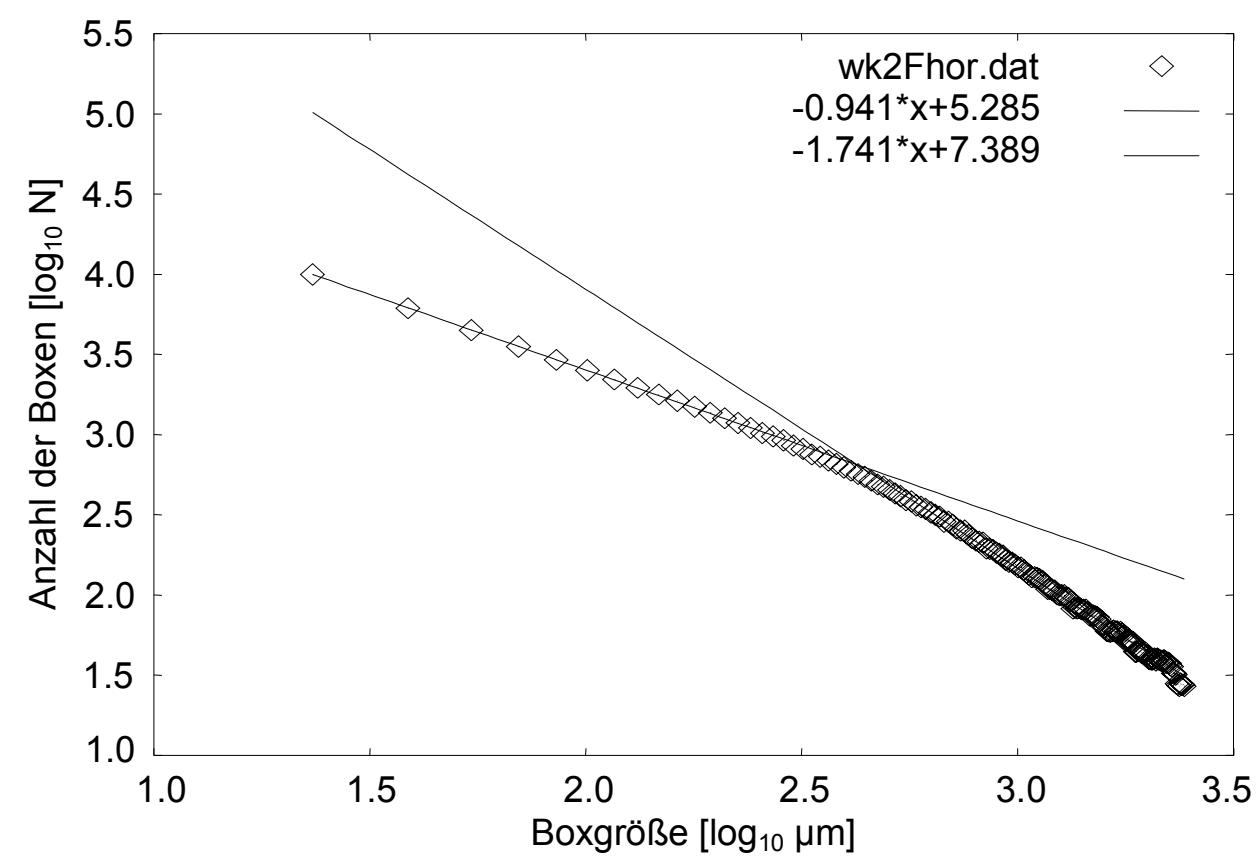

Abb. 6.11: Anpassung von zwei Geraden an die mit dem Boxcounting-Verfahren gewonnenen Daten (Mittelwerte von 7 Bildern) für die lichtmikroskopischen Aufnahmen der horizontal ausgerichteten Dünnschliffe einer Kubiena-Box. 


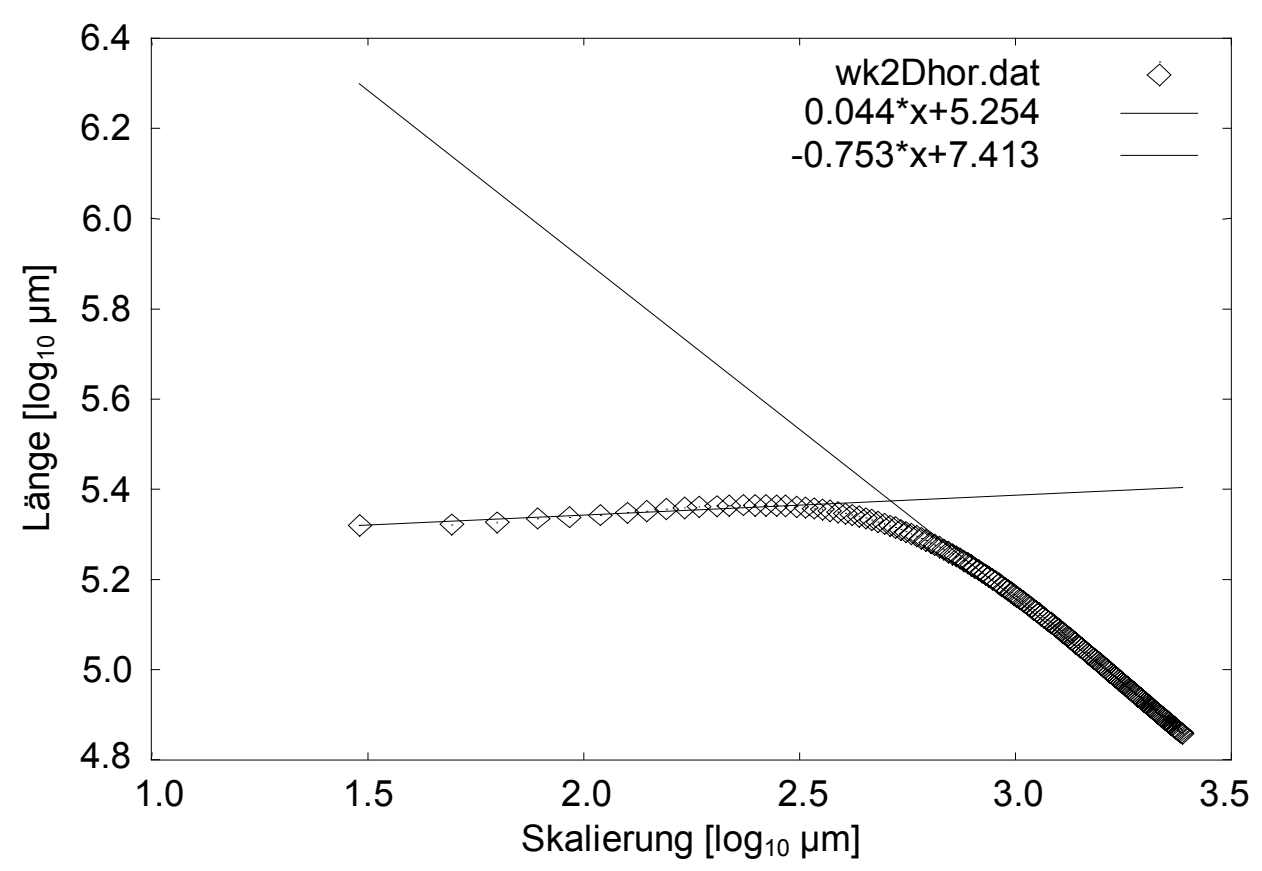

Abb. 6.12: Die gleichen Aufnahmen wie für Abb. 6.11, gemessen mit Dilatation.

Wie für die Einzelbilder in Kapitel 6.1.2.1 beschrieben, wurden auch für die vertikal und horizontal ausgerichteten Dünnschliffe die D für den jeweiligen optimalen Bereich innerhalb des unteren und oberen Cutoffs berechnet. Die Ergebnisse sind in Tab. 6.10 dargestellt. $\mathrm{D}^{\mathrm{d}}$ ist kleiner als $\mathrm{D}^{\mathrm{b}}$, wobei die Schwankungsbreite gering ist: Die Werte bewegen sich zwischen $\mathrm{D}^{\mathrm{d}}=1,770$ für WK2ver und $\mathrm{D}^{\mathrm{b}}=1,779$ für WK2hor, die Differenzen für Boxcounting sind etwas geringer als die Standardfehler, für Dilatation entsprechen sie ihm.

Tab. 6.10: Fraktale Dimensionen und für deren Berechnung relevante Parameter der Serie Wild Klotz 2. Die Berechnung erfolgte differenziert in horizontale und vertikale Schliffausrichtungen aus den gemittelten Rohdaten (Methode B) gemessen an mit dem Wild-Photomakroskop aufgenommenen Einzelbildern. $\mathrm{n}$ (B) ist die Anzahl der Datenpaare und se der Standardfehler des Regressionskoeffizienten für die Steigung. D wurde für jedes Bild und jede Methode aus dem optimalen linearen Bereich des Richardson-Plots bestimmt.

\begin{tabular}{|c|c|c|c|c|c|c|}
\hline Serie & Methode & $\begin{array}{l}\text { Auflösung } \\
\text { [um/Pixel] }\end{array}$ & $\begin{array}{c}\text { unterer } \\
\text { Cutoff }[\mu \mathrm{m}]\end{array}$ & $\begin{array}{c}\text { oberer } \\
\text { Cutoff }[\mu \mathrm{m}]\end{array}$ & $\mathrm{n}(\mathrm{B})$ & D mit se \\
\hline \multirow{2}{*}{ WK2 ver } & Boxcounting & \multirow{2}{*}{7,763} & 566,70 & 2429,82 & 121 & $1,771(0,011)$ \\
\hline & Dilatation & & 776,60 & 2717,23 & 126 & $1,770(0,003)$ \\
\hline \multirow{2}{*}{ WK2 hor } & Boxcounting & \multirow{2}{*}{7,763} & 489,07 & 2429,82 & 126 & $1,779(0,010)$ \\
\hline & Dilatation & & 667,57 & 2717,23 & 133 & $1,773(0,003)$ \\
\hline
\end{tabular}

Um festzustellen, ob es einen signifikanten Unterschied für die mit den verwendeten Verfahren gemessene Komplexität der Struktur (am Beispiel der Porenraumgrenzlinie) zwischen vertikal und horizontal ausgerichteten Schliffen gibt, wurde ein T-Test durchgeführt. Dafür wurde der gesamte Skalenbereich mit Ausnahme der Messung mit einer Sondengröße von 1 Pixel berücksichtigt, von 23,29 bis 2429,82 $\mu \mathrm{m}$ für Boxcounting und bis 2717,23 $\mu \mathrm{m}$ für Dilatation. Die Messwerte beider Methoden wurden getrennt verglichen, wobei je eine Gerade über den gesamten Bereich angepasst wurde. Das 
Ergebnis ist eindeutig: $\mathrm{D}$ (hor) $>\mathrm{D}$ (ver) mit einer Irrtumswahrscheinlichkeit von $\mathrm{p}<10^{-6}$ für Boxcounting und $\mathrm{p}<10^{-8}$ für Dilatation.

Die Ergebnisse für die Einzelbilder Wild7 und Wild8 hingegen stellen genau den umgekehrten Sachverhalt dar: In Tab. 6.8 ist zwar ebenfalls $\mathrm{D}^{\mathrm{d}}<\mathrm{D}^{\mathrm{b}}$, aber $\mathrm{D}($ Wild7) $>\mathrm{D}$ (Wild8), und somit die Komplexität des vertikal ausgerichteten Schliffes höher als die des horizontal ausgerichteten. Der T-Test wurde wie für die WK2-Serien für Geradenanpassungen über den gleichen Skalierungsbereich durchgeführt und ergibt $D($ Wild7) $>$ D(Wild8), allerdings auf einem Signifikanzniveau $<99,9 \%$, und zwar 0,983 für Boxcounting und 0,972 für Dilatation.

In allen bis hier gezeigten Tabellen zeigt sich die Abhängigkeit der berechneten fraktalen Dimension von dem Größenbereich der Strukturlemente innerhalb der Cutoffs. Im folgenden Kapitel soll daher die Abhängigkeit der fraktalen Dimension von der Auflösung untersucht werden, da die Problematik eng mit der Wahl der Cutoffs verknüpft ist.

\subsubsection{Abhängigkeit der fraktalen Dimension von der Bildauflösung}

Wie im Methodenteil unter 4.6.3 beschrieben wurden zwei Bildausschnitte der Serie Leo8 mit Vergrößerungen von 150x und 500x und je vier verschiedenen Auflösungen abgespeichert und mit dem Boxcountingverfahren gemessen. Mittels des unter Kapitel 5.1.1.1 beschriebenen Verfahrens der besten linearen Regression wurde der optimale Datenbereich für jede Auflösungsstufe einzeln bestimmt. Dann wurde ein für alle Stufen einheitlicher Skalierungsbereich festgelegt und die so ermittelten fraktalen Dimensionen verglichen (s. Tab. 6.11 und 6.12).

Wird der optimale Datenbereich für jede Auflösungsstufe einzeln bestimmt, ändert sich der Wert für D der Vergrößerung 150x für höhere Auflösungen lediglich um $\Delta=0,002$. Wird ein einheitlicher Datenbereich festgelegt, wächst D mit der Auflösung um maximal $\Delta=0,024$ (s. Tab. 6.11). 
Tab. 6.11: Fraktale Dimensionen und für deren Berechnung relevante Parameter eines mit 150x Vergrößerung und verschiedenen Auflösungen am FE-REM aufgenommenen Einzelbildes der Serie Leo8. n (B) ist die Anzahl der Datenpaare und se der Standardfehler des Regressionskoeffizienten für die Steigung. D wurde nach zwei Verfahren bestimmt: (i) Für jede Auflösung aus dem optimalen linearen Bereich des Richardson-Plots (oben) und (ii) für einen gemeinsamen Skalenbereich innerhalb der Cutoffs (unten).

\begin{tabular}{cccccc}
\hline $\begin{array}{c}\text { Bildgröße } \\
{\left[\text { Pixel }^{2}\right]}\end{array}$ & $\begin{array}{c}\text { Auflösung } \\
{[\mu \mathrm{m} / \text { Pixel] }}\end{array}$ & $\begin{array}{c}\text { unterer Cutoff oberer Cutoff } \\
{[\mu \mathrm{m}]}\end{array}$ & $\begin{array}{c}\mathrm{n}(\mathrm{B}) \\
{[\mu \mathrm{m}]}\end{array}$ & D mit se \\
\hline $512 \times 345$ & 3,333 & 23,33 & 83,33 & 10 & $1,953(0,012)$ \\
\hline $1024 \times 690$ & 1,724 & 18,97 & 108,62 & 27 & $1,940(0,007)$ \\
\hline $2048 \times 1380$ & 0,870 & 16,52 & 92,26 & 48 & $1,939(0,006)$ \\
\hline $3072 \times 2304$ & 0,592 & 17,16 & 75,15 & 50 & $1,938(0,005)$ \\
\hline $\begin{array}{c}\text { Bildgröße } \\
{\left[\text { Pixel }^{2}\right]}\end{array}$ & $\begin{array}{c}\text { Auflösung } \\
{[\mu \mathrm{m} / \text { Pixel] }}\end{array}$ & $\begin{array}{c}\text { unterer Cutoff } \\
{[\mu \mathrm{m}]}\end{array}$ & $\begin{array}{c}\text { oberer Cutoff } \\
{[\mu \mathrm{m}]}\end{array}$ & $\mathrm{n}(\mathrm{B})$ & $\mathrm{D}$ mit se \\
\hline $512 \times 345$ & 3,333 & 16,66 & 76,66 & 10 & $1,914(0,016)$ \\
\hline $1024 \times 690$ & 1,724 & 15,52 & 74,14 & 18 & $1,927(0,009)$ \\
\hline $2048 \times 1380$ & 0,870 & 16,52 & 75,65 & 35 & $1,937(0,007)$ \\
\hline $3072 \times 2304$ & 0,592 & 17,16 & 75,15 & 50 & $1,938(0,005)$ \\
\hline
\end{tabular}

Tab. 6.12: Wie Tab. 6.11 für ein mit 500x Vergrößerung und verschiedenen Auflösungen am FE-REM aufgenommenes Einzelbild der Serie Leo8.

\begin{tabular}{cccccc}
\hline $\begin{array}{c}\text { Bildgröße } \\
{\left[\text { Pixel }^{2}\right]}\end{array}$ & $\begin{array}{c}\text { Auflösung } \\
{[\mu \mathrm{m} / \text { Pixel] }}\end{array}$ & $\begin{array}{c}\text { unterer Cutoff oberer Cutoff } \\
{[\mu \mathrm{m}]}\end{array}$ & $\begin{array}{c}\mathrm{n}(\mathrm{B}) \\
{[\mu \mathrm{m}]}\end{array}$ & D mit se \\
\hline $512 \times 345$ & 1,000 & 9,00 & 63,00 & 28 & $1,877(0,011)$ \\
\hline $1024 \times 690$ & 0,513 & 8,72 & 42,56 & 34 & $1,875(0,012)$ \\
\hline $2048 \times 1380$ & 0,260 & 9,61 & 37,14 & 54 & $1,882(0,009)$ \\
\hline $3072 \times 2304$ & 0,177 & 7,61 & 35,22 & 79 & $1,913(0,005)$ \\
\hline $\begin{array}{c}\text { Bildgröße } \\
{\left[\text { Pixel }^{2}\right]}\end{array}$ & $\begin{array}{c}\text { Auflösung } \\
{[\mu \mathrm{m} / \text { Pixel] }}\end{array}$ & $\begin{array}{c}\text { unterer Cutoff } \\
{[\mu \mathrm{m}]}\end{array}$ & $\begin{array}{c}\text { oberer Cutoff } \\
{[\mu \mathrm{m}]}\end{array}$ & $\mathrm{n}(\mathrm{B})$ & $\mathrm{D}$ mit se \\
\hline $512 \times 345$ & 1,000 & 9,00 & 35,00 & 14 & $1,873(0,020)$ \\
\hline $1024 \times 690$ & 0,513 & 8,72 & 35,38 & 27 & $1,860(0,013)$ \\
\hline $2048 \times 1380$ & 0,260 & 9,09 & 35,06 & 51 & $1,866(0,009)$ \\
\hline $3072 \times 2304$ & 0,177 & 9,03 & 35,22 & 75 & $1,920(0,005)$ \\
\hline
\end{tabular}



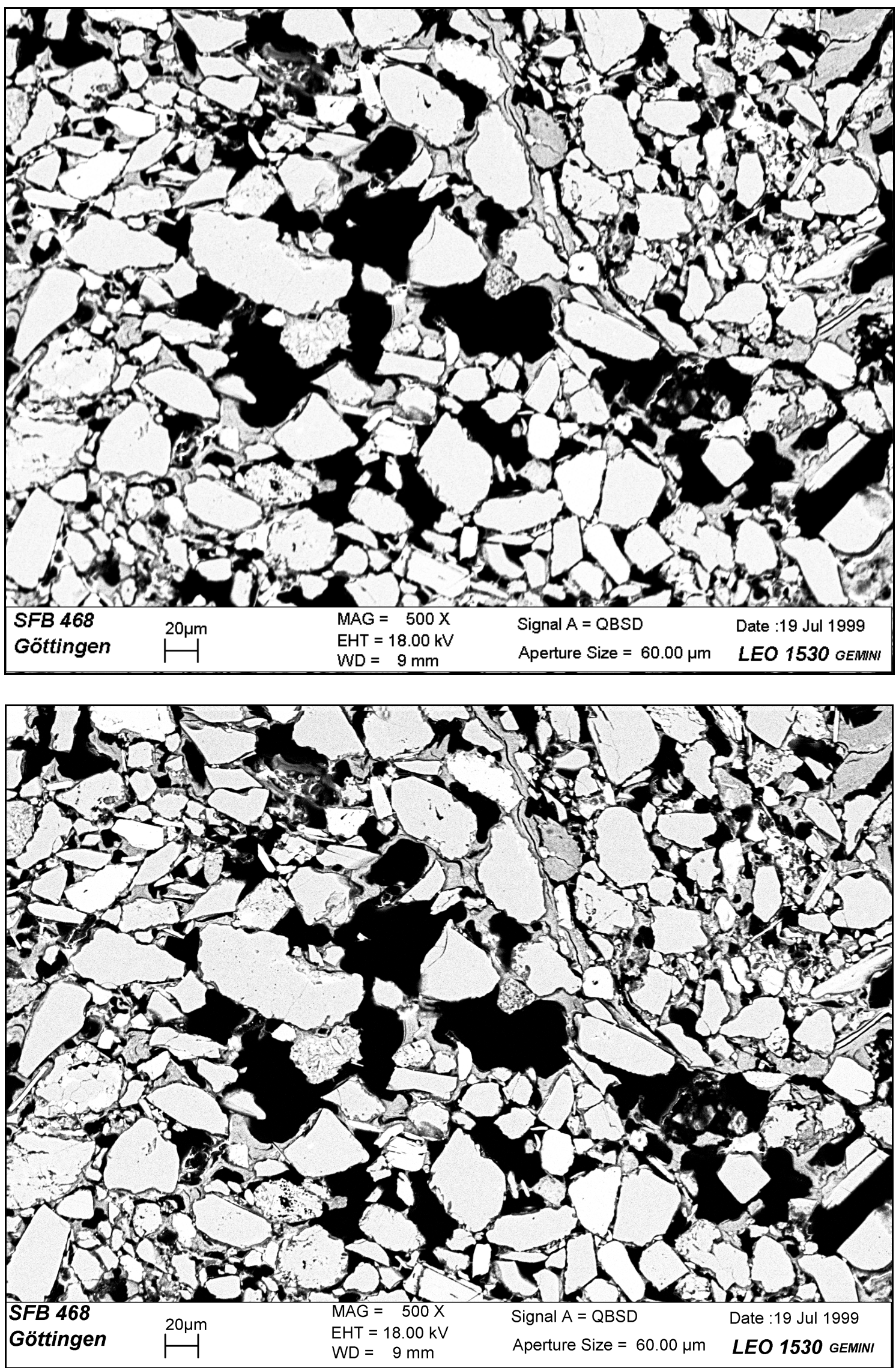

Abb. 6.13: Hier macht sich nicht nur die unterschiedliche Auflösung bemerkbar (oben: 1024 x 690 Pixel, unten: 3072 x 2304 Pixel, jeweils ohne die Legende), sondern auch der höhere Kontrast im unteren Bild. Dadurch sind kleinere Details deutlicher erkennbar und die fraktale Dimension nimmt zu. 
Für die mit Vergrößerung 500x aufgenommenen Bilder ist kein so eindeutiger Trend erkennbar. Vor allem das vierte, mit der höchsten Auflösungsstufe gespeicherte Bild weist eine deutlich höhere fraktale Dimension als die übrigen drei auf (Tab. 6.12). Der Grund ist nicht die höhere Auflösung, sondern ein bei der Bildaufnahme entstandener höherer Kontrast, der dazu führt, dass kleinere Strukturen deutlicher erkennbar werden (Abb. 6.13). Bei den übrigen drei Auflösungsstufen macht sich vorrangig die Wahl des Datenbereichs bemerkbar. Die Differenz für die niedrigste Auflösungsstufe fällt mit $\Delta=0,004$ am geringsten aus, obwohl der obere Cutoff bei der Optimierung für jedes einzelne Bild deutlich höher liegt als bei einem einheitlichen Datenbereich für alle Auflösungsstufen. Die zweite und dritte Auflösungsstufe liefern sowohl für den optimalen als auch für den einheitlichen Skalierungsbereich ein höheres D für die bessere Auflösung, wobei die Differenz $(\Delta=0,007$ bzw. $\Delta=0,006)$ innerhalb des Standardfehlers liegt. Hier macht sich der Skalierungsbereich deutlicher bemerkbar, ein kleinerer oberer Cutoff führt zu einem kleineren D, die Differenzen sind größer als die jeweiligen Standardfehler $(\Delta=0,015$ für die Auflösung 1024x690 Pixel und $\Delta=0,016$ für die Auflösung 2048x1380 Pixel).

Aus den Tab. 6.11 und 6.12 ergeben sich folgende Erkenntnisse: (i) die Wahl eines optimalen linearen Bereichs im log-log-Plot führt unabhängig von der Auflösung zu vergleichbaren Ergebnissen und (ii) die Wahl des Cutoffs hat für höhere Vergrößerungsstufen eine größere Bedeutung als die Wahl der Auflösung. Daher kann die zweitniedrigste (1024 x 690 Pixel) Auflösungsstufe als hinreichend genau angesehen werden, die für Leo9 verwendete dritte Stufe (2048 x 1380) führt zu langen Laufzeiten der Messprogramme und beim Dilatationsverfahren an die Grenzen der verwendeten Software - die Grenzlinie wird so komplex abgebildet, dass der interne Speicher im KS400 nicht ausreicht oder das Programm beim Bestimmen der Verzweigungsrichtungen (s. Abschnitt 4.6.2) abbricht. Die Abhängigkeit der fraktalen Dimension von der Vergrößerungsstufe bzw. von der Wahl der Cutoffs scheint nur untergeordnet eine Funktion der Auflösung zu sein. Diese Problematik wird im folgenden Kapitel behandelt.

\subsection{Abhängigkeit der fraktalen Dimension von der Auflösung bei verschiedenen Vergrößerungsstufen}

Wie in den vorangegangenen Kapiteln dargelegt, wächst die fraktale Dimension der Porenraumgrenzlinie und damit die Komplexität der REM-Aufnahmen mit abnehmender Vergrößerungsstufe. Zunächst wurde der Zusammenhang zwischen dem mittleren logarithmischen Cutoff und der Bildauflösung untersucht (Alex McBratney, mündliche Mitteilung). Durch lineare Regression ergeben sich die folgenden Geradengleichungen, wobei die in den Tab. 6.1 bis 6.3 gezeigten Cutoffs und Auflösungen mit allen verfügbaren Nachkommastellen verwendet wurden.

Tab. 6.13: Zusammenhang zwischen dem mittleren logarithmischen Cutoff (y) und der für die jeweilige Vergrößerungsstufe verwendeten Auflösung (x).

\begin{tabular}{ccc}
\hline Serie & Geradengleichung & Regressionskoeffizient \\
\hline Leo7 & $\mathrm{y}=28,466 \mathrm{x}+3,204$ & $\mathrm{r}^{2}=0,9915$ \\
\hline Leo8 & $\mathrm{y}=28,632 \mathrm{x}+2,142$ & $\mathrm{r}^{2}=0,9984$ \\
\hline Leo9 & $\mathrm{y}=48,804 \mathrm{x}-0,027$ & $\mathrm{r}^{2}=0,9952$ \\
\hline
\end{tabular}


In Abb. 6.14 ist dieser Zusammenhang für Leo8 graphisch dargestellt.

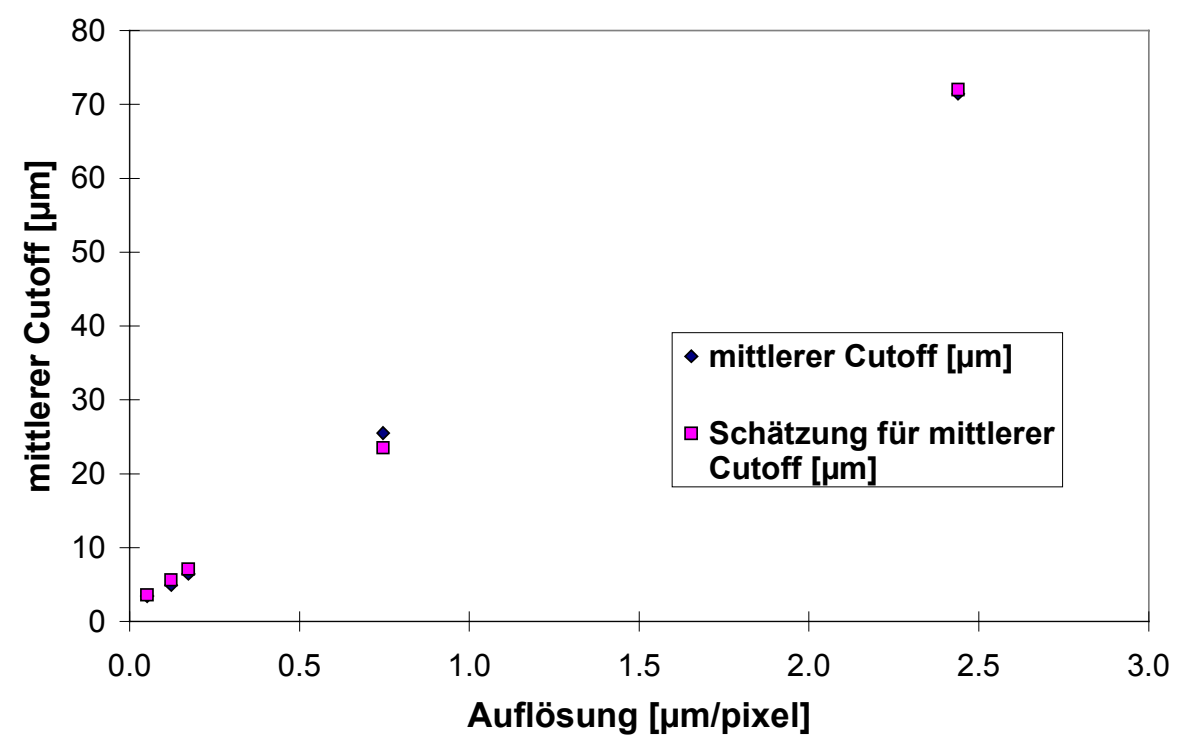

Abb. 6.14: Der mittlere Cutoff (als logarithmisches Mittel zwischen dem unteren und oberen Cutoff jeder Vergrößerungsstufe) am Beispiel von Leo8, dargestellt über der Bildauflösung sowie die mit linearer Regression geschätzten mittleren Cutoffs (s. Tab. 6.13).

Im nächsten Schritt wurde für jede Messserie und Methode eine einfache Sättigungsfunktion (Boltzmann-Funktion) angepasst.

$$
\mathrm{D}_{\mathrm{S}}=\mathrm{D}_{\mathrm{Smin}}+\left(\mathrm{D}_{\mathrm{Smax}}-\mathrm{D}_{\mathrm{Smin}}\right)\left(1-\mathrm{e}^{-\varepsilon \mathrm{k}}\right)
$$

Diese Funktion bietet den Vorteil, dass in Abhängigkeit von der Auflösung $\varepsilon$ eine minimale $\left(\mathrm{D}_{\mathrm{Smin}}\right)$ und eine maximale $\left(\mathrm{D}_{\mathrm{Smax}}\right)$ fraktale Dimension angepasst werden können. Der Index S steht für „surface“ und beinhaltet die Annahme, dass die in einer Fläche gemessene fraktale Dimension einer Länge $\left(\mathrm{D}_{\mathrm{L}}\right)$ durch die Addition von 1 die fraktale Dimension einer Oberfläche $\left(D_{S}\right)$ im Raum darstellt (Mandelbrot 1983, Kapitel 14).

$$
\mathrm{D}_{\mathrm{S}}=\mathrm{D}_{\mathrm{L}}+1
$$

Streng genommen gilt dies nur, wenn der dreidimensionale Körper in hinreichend vielen Raumrichtungen geschnitten und dann ein Mittelwert gebildet wird. Wie die Auswertung verschiedener Schliffrichtungen zeigte, sind die Unterschiede bzgl. der Fraktalität tatsächlich klein, wenngleich signifikant (s. Kap. 6.1.2.2). Daher erscheint die Verwendung der obigen Gleichung gerechtfertigt.

Die Konstante k in Gl. 6.1 ist eine Maßzahl dafür, wie schnell bzw. bei welcher Auflösung $D_{\text {Smax }}$ erreicht wird. Je größer k, desto steiler verläuft der Anstieg der Kurve im quasilinearen Bereich. Für $\varepsilon \rightarrow 0$ geht $\mathrm{e}^{-\varepsilon \mathrm{k}} \rightarrow 1$, so dass für kleine Auflösungen $\mathrm{D}_{\text {Smin }}$ erreicht wird. Für $\varepsilon \geq 2,5 \mu \mathrm{m}$ (die geringste verwendete Auflösung bei den REM-Aufnahmen) und das entsprechende $\mathrm{k}$ geht $\mathrm{e}^{-\mathrm{k}} \rightarrow 0$, so dass sich für geringe Auflösungen (bzw. große $\varepsilon$ ) $\mathrm{D}_{\text {Smax }}$ ergibt. In Abb. 6.15 sind die Anpassungen für Leo7 und Leo8, Boxcounting-Verfahren, sowie für alle Messserien dargestellt, die Werte für die Parameter finden sich in Tab. 6.14. Die entsprechenden Abbildungen für das Dilatationsverfahren sowie für Leo9, Boxcounting und Dilatation, befinden sich im Anhang unter A.10 und A.11. 

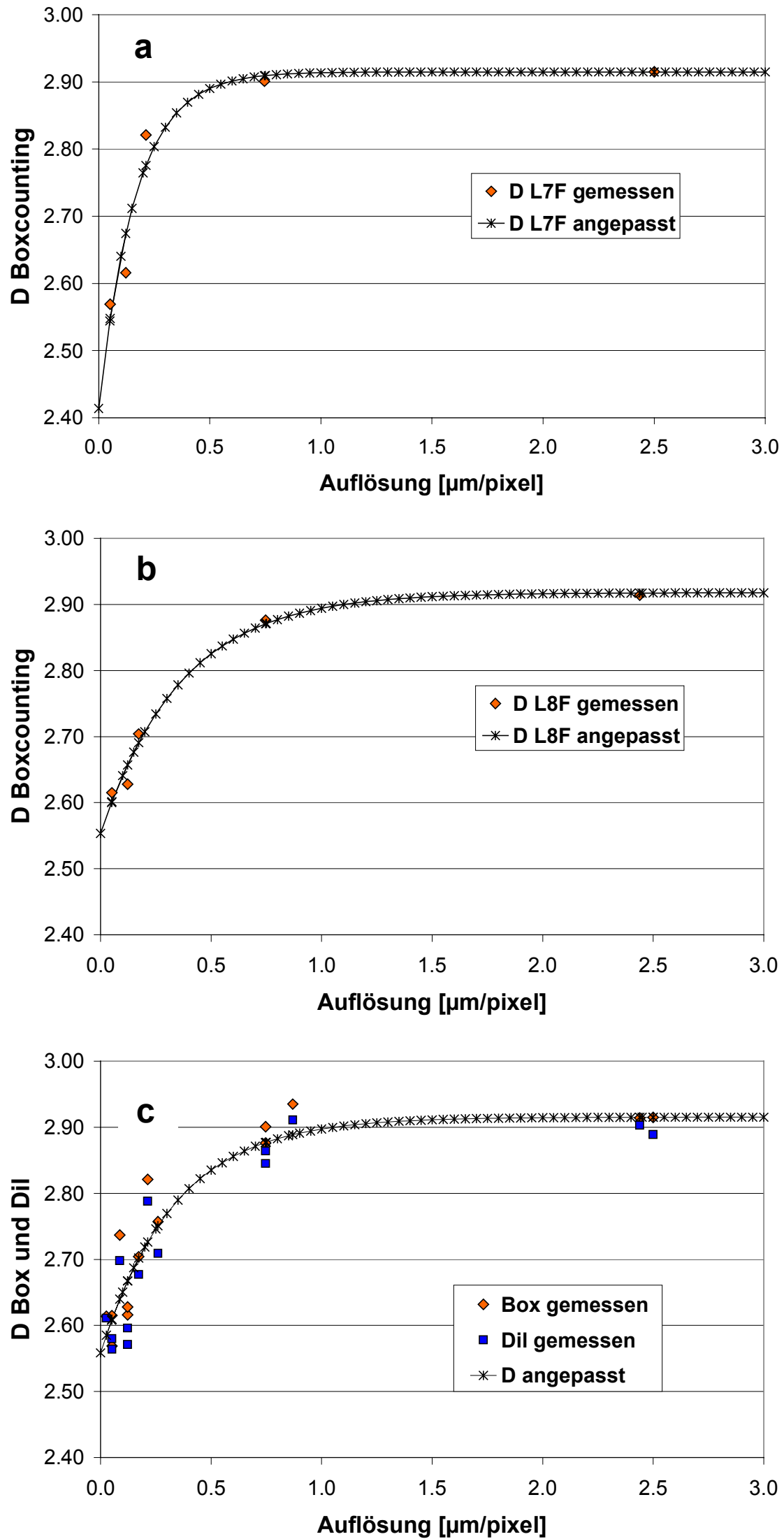

Abb. 6.15: Abhängigkeit der gemessenen fraktalen Dimension von der Auflösung bzw. Vergrößerungsstufe. (a) Graphische Darstellung für Leo7, Boxcounting; (b) für Leo8, Boxcounting und (c) für alle drei Messserien und die Methoden Boxcounting und Dilatation. Die Parameter der Gl. 6.1 sind in Tab. 6.14 zusammengestellt. 
Tab. 6.14: Mit dem Levenberg-Marquard-Verfahren und einer Optimierung durch Minimierung der Abweichungsquadrate geschätzte Parameter für die minimale $\left(\mathrm{D}_{\text {Smin }}\right)$ und die maximale $\left(\mathrm{D}_{\text {Smax }}\right)$ fraktale Dimension sowie den Anpassungsparameter k. Als Kriterium für die Güte der Anpassung ist der Regressionskoeffizient angegeben.

\begin{tabular}{cccccc}
\hline Serie & Methode & $D_{\text {Smin }}$ & $D_{\text {Smax }}$ & $k[$ Pixel/ $\mu \mathrm{m}]$ & Regressionskoeffizient \\
\hline \multirow{2}{*}{ Leo7 } & Boxcounting & 2,414 & 2,915 & 6,017 & $\mathrm{r}^{2}=0,9429$ \\
\cline { 2 - 6 } & Dilatation & 2,429 & 2,886 & 5,185 & $\mathrm{r}^{2}=0,9093$ \\
\hline \multirow{2}{*}{ Leo8 } & Boxcounting & 2,554 & 2,918 & 2,743 & $\mathrm{r}^{2}=0,9840$ \\
\cline { 2 - 6 } & Dilatation & 2,561 & 2,911 & 1,984 & $\mathrm{r}^{2}=0,9720$ \\
\hline \multirow{2}{*}{ Leo9 } & Boxcounting & 2,627 & 2,993 & 2,062 & $\mathrm{r}^{2}=0,9233$ \\
\cline { 2 - 6 } & Dilatation & 2,611 & 3,000 & 1,548 & $\mathrm{r}^{2}=0,9423$ \\
\hline \multirow{2}{*}{ alle } & Box u. Dil & 2,558 & 2,915 & 2,984 & $\mathrm{r}^{2}=0,9997$ \\
\hline
\end{tabular}

Von praktischem Interesse ist nun der Punkt bzw. die Bildauflösung, für den $\mathrm{D}_{\max }$ mit hinreichender Genauigkeit erreicht ist. Eine Genauigkeit in der Größenordnung der dritten Nachkommastelle wird als hinreichend erachtet, so dass gefordert wird

$$
\mathrm{e}^{-\varepsilon \mathrm{k}} \leq 0,001=\mathrm{e}^{\ln 0,001}
$$

Daraus folgt

$$
-\mathrm{k} \varepsilon=\ln 0,001 \approx 6,9078 .
$$

Der Parameter k wurde für jede Messserie berechnet (s. Tab. 6.14), so dass die optimale Auflösung $\varepsilon_{\text {opt }}$ bestimmt werden kann. Der entsprechende kritische Cutoff kann nach den Regressionsgleichungen aus Tab. 6.13 ebenfalls angegeben werden. Er ist als die Boxgröße bzw. der Durchmesser der Umhüllenden bei Dilatation zu sehen, bei der $\mathrm{D}_{\max }$ annährend erreicht ist, (s. Tab. 6.15).

Tab. 6.15: Nach den Gl. 6.3 und 6.4 berechnete optimale Auflösung $\varepsilon_{\text {opt }}$ und der kritische Cutoff, bei dem die maximale fraktale Dimension $\mathrm{D}_{\max }$ erreicht ist.

\begin{tabular}{cccc}
\hline Serie & Methode & $\varepsilon_{\text {opt }}[\mu \mathrm{m} /$ Pixel] & $\begin{array}{c}\text { kritischer } \\
\text { Cutoff }[\mu \mathrm{m}]\end{array}$ \\
\hline \multirow{2}{*}{ Leo7 } & Boxcounting & 1,148 & 35,885 \\
\cline { 2 - 4 } & Dilatation & 1,332 & 41,130 \\
\hline \multirow{2}{*}{ Leo8 } & Boxcounting & 2,518 & 74,239 \\
\cline { 2 - 4 } & Dilatation & 3,482 & 101,829 \\
\hline \multirow{2}{*}{ Leo9 } & Boxcounting & 3,349 & 163,438 \\
\cline { 2 - 4 } & Dilatation & 4,463 & 217,777 \\
\hline
\end{tabular}

Wie auch aus den Abb. 6.15 und A.10 und A.11 zu erkennen ist, wird $\mathrm{D}_{\max }$ der Serie Leo7 bereits bei einer recht kleinen Auflösung von 1,148 bis 1,332 $\mu \mathrm{m} /$ Pixel erreicht, was im Bereich der ersten und zweiten Vergrößerungsstufe liegt (s. Tab. 6.1). Die für die Serie Leo8 gemessenen fraktalen Dimensionen erreichen $\mathrm{D}_{\max }$ bereits im Bereich der ersten 
Vergrößerungsstufe (geringste Auflösung, s. Tab. 6.2) und für Leo9 unterhalb der verwendeten Auflösungsstufen (s. Tab. 6.3). Die Anpassungskurven der G1. 6.1 verlaufen für Dilatation immer flacher als für das Boxcounting-Verfahren. Bei Leo9, Dilatation, wird der vorgegebene Grenzwert von $D_{\max }=3,00$ erreicht. Weitere Anmerkungen zu dem verwendeten Parameterschätzverfahren finden sich in der Diskussion unter 7.3.

Zusammenfassend sei festgehalten, dass die hier gemessene fraktale Dimension auch eine Funktion der Größe des Strukurelementes bzw. der Bildauflösung darstellt. Für den Richardson-Plot ergibt sich empirisch aus den Gl. 5.1 und 6.1 für das BoxcountingVerfahren

$$
\log N\left(\varepsilon_{\mathrm{p}}, \varepsilon_{\mathrm{s}}\right)=-\left(\mathrm{D}_{\text {min }}^{\mathrm{b}}+\left(\mathrm{D}_{\text {max }}^{\mathrm{b}}-\mathrm{D}_{\text {min }}^{\mathrm{b}}\right)\left(1-\mathrm{e}^{-\varepsilon_{\mathrm{p}} \mathrm{k}}\right)\right) \log \varepsilon_{\mathrm{s}}+\mathrm{c}
$$

bzw. aus den G1. 5.2 und 6.1 für das Dilatationsverfahren

$$
\log L\left(\varepsilon_{\mathrm{p}}, \varepsilon_{\mathrm{s}}\right)=1-\left(\mathrm{D}_{\min }^{\mathrm{d}}+\left(\mathrm{D}_{\max }^{\mathrm{d}}-\mathrm{D}_{\text {min }}^{\mathrm{d}}\right)\left(1-\mathrm{e}^{-\varepsilon_{\mathrm{p}} \mathrm{k}}\right)\right) \log \varepsilon_{\mathrm{s}}+\mathrm{c}
$$

Diese Gleichungen weisen zusätzlich zu der üblicherweise verwendeten Abhängigkeit der Messgröße von der Größe des skalierenden Elementes $\varepsilon_{\mathrm{s}}$ eine Abhängigkeit von der physikalischen Pixelgröße $\varepsilon_{\mathrm{p}}$ auf. Da $\varepsilon_{\mathrm{s}}$ und $\varepsilon_{\mathrm{p}}$ laut den in Tab. 6.13 gezeigten Geradengleichungen eine lineare Abhängigkeit aufweisen, stellt $\varepsilon_{\mathrm{p}}$ keinen zusätzlichen Parameter dar. Für die Anpassungen nach G1. 6.1 kann auch $\varepsilon_{\mathrm{s}}$ verwendet werden, dann ändert sich die Skalierung der x-Achse in Abb. 6.15 und der Wert für k entsprechend.

\subsection{Ergebnisse der Messung der Porengrößenverteilung}

Das verwendete Programm liefert als Ergebnis für jeden Opening-Schritt die Fläche aller Poren, die kleiner als der Durchmesser des verwendeten skalierenden Elements sind. Dies ist in Abb. 6.16 exemplarisch dargestellt. Poren oder Porenbereiche, die einem sukzessive wachsenden definierten Durchmesser zugeordnet werden können, sind in derselben Farbe markiert. In den Ergebnistabellen erscheint für jeden Opening-Schritt die Fläche der zugeordneten Poren. Die ebenfalls aufgenommene Anzahl der Poren wurde zunächst nicht weiter verarbeitet. 


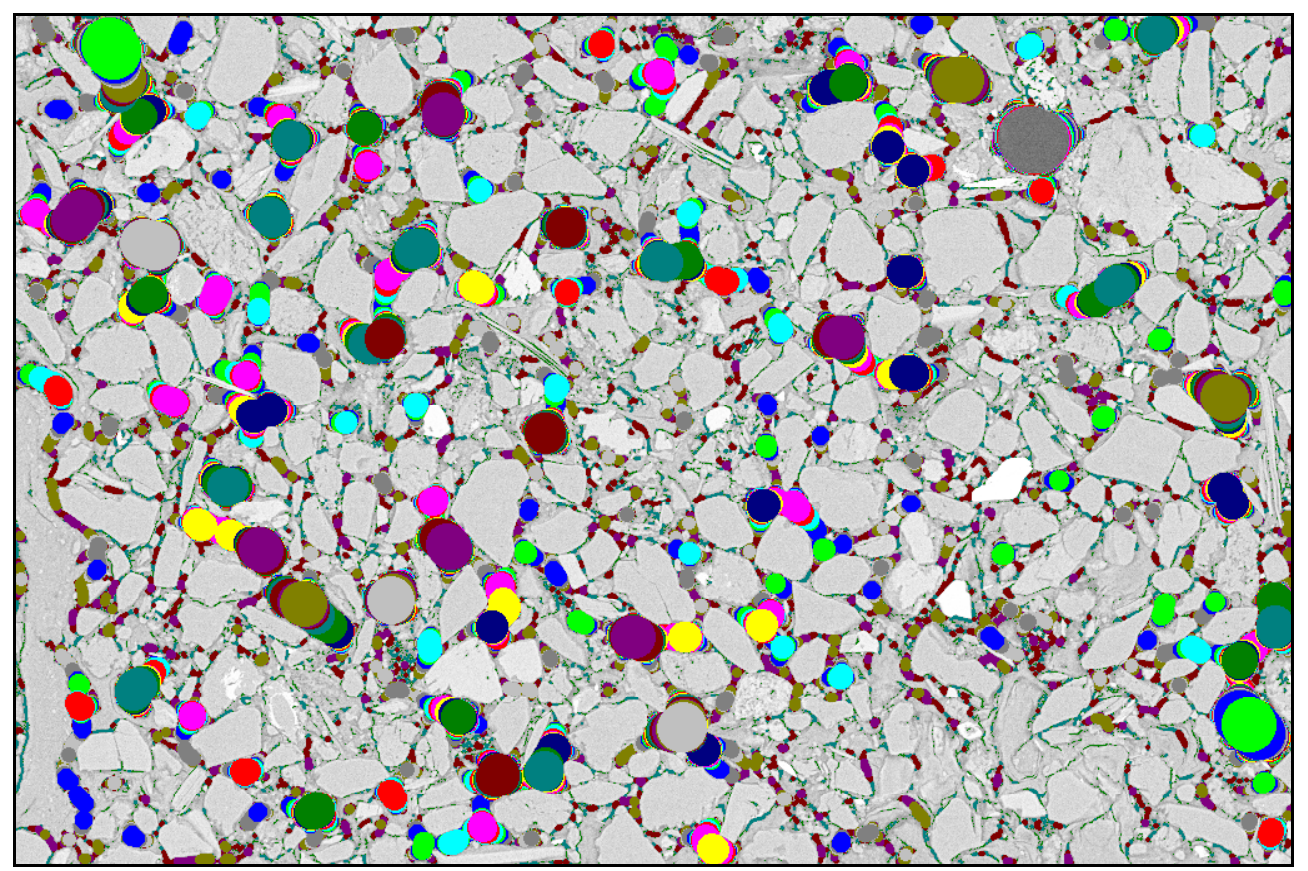

Abb. 6.16: Ergebnisbild des Opening-Verfahrens über dem Originalbild. Es handelt sich um eine Aufnahme der Serie Leo8, 500x Vergrößerung (s. Abb. 4.2 und 4.10). Poren gleichen Durchmessers sind mit der gleichen Farbe markiert. Da nur eine begrenzte Anzahl Farben zur Verfügung steht, kommen Wiederholungen vor (z. B. für hellgrün).

Beim Vergleich der Häufigkeiten verschiedener Auflösungsstufen muss beachtet werden, dass die Fläche der Prozentangabe proportional zur Häufigkeit ist und nicht ihre Höhe. Bei unterschiedlichen Klassenbreiten muss also durch die Klassenbreite dividiert werden (s. Stahel 2000, Kapitel 2). In Abb. 6.17 sind die Porenhäufigkeiten zunächst für jede Vergrößerungsstufe einzeln dargestellt.

\subsubsection{Messungen an FE-REM Aufnahmen}

Die distributive Porengrößenverteilung ist hier für die Serie Leo7 (s. Abb. 6.17) dargestellt. Es handelt sich um Mittelwerte der Porenflächenhäufigkeiten von je 19 bis 21 Bildern pro Auflösungsstufe und Durchmesser des skalierenden Elementes. Die Ergebnisse für Leo8 sind im Anhang als Abb. A.12 dargestellt. Dort finden sich in den Tab. A.1 und A.2 detaillierte Angaben über die Auflösung, die Anzahl der Bilder, die physikalische Bildgröße, die jeweils größte und kleinste erfasste Pore sowie die größte Pore die auf der Hälfte bzw. allen Aufnahmen vorkommt und die kleinste Pore, bei der der Variationskoeffizient $<100 \%$ bleibt. Der letzte Wert wurde benutzt, um die kumulative Porengrößenverteilung analog zur Retentionskurve darstellen zu können. In den Abb. 6.18 und 6.19 sind die Werte von den kleinsten bis zu den größten erfassten Poren dargestellt.

Bei der Betrachtung der als Verteilungskurven dargestellten distributiven Porengrößen fällt auf, dass die kleinen Poren jeweils am häufigsten vorkommen und erst bei der höchsten Vergrößerungsstufe ein Maximum bei einem Porendurchmesser von 1,5 $\mu \mathrm{m}$ deutlich sichtbar wird. Mit zunehmender Auflösung streuen die Werte von Messung zu Messung immer mehr. Die einzelnen Spitzen in Abb. 6.17e sind keine Messfehler, sondern proportional zum Bildausschnitt recht große Poren einzelner Bilder, die gemessen werden, wenn die entsprechende Größe des skalierenden Elementes erreicht ist. 

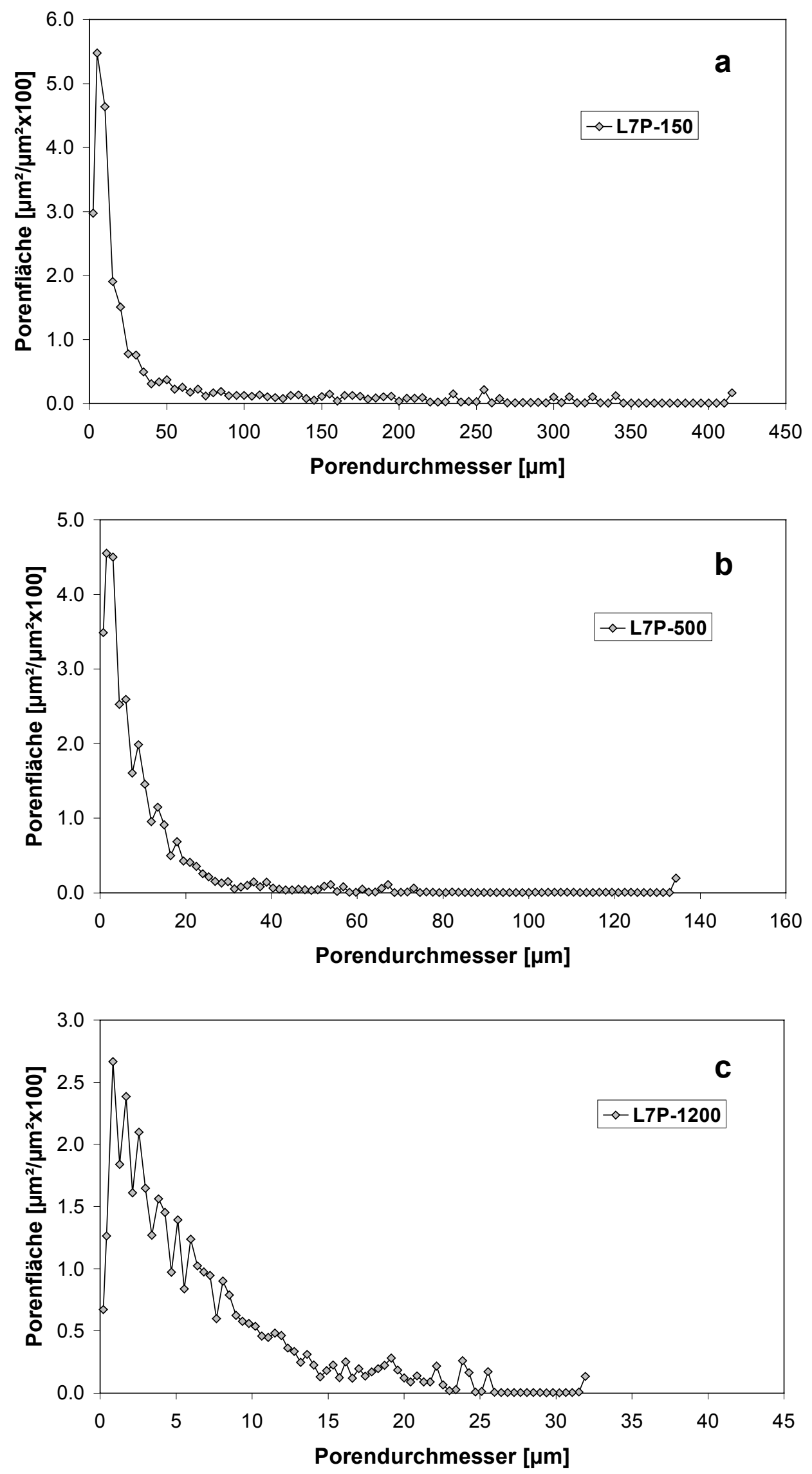

Abb. 6.17: Distributive Porengrößenverteilung der Serie Leo7. Dargestellt sind die Mittelwerte der Porenflächenhäufigkeiten von je 19 bis 21 Bildern pro Auflösungsstufe und Durchmesser des skalierenden Elementes. Angaben zu den Datenbereichen sind in Tab. A.1 aufgelistet. 

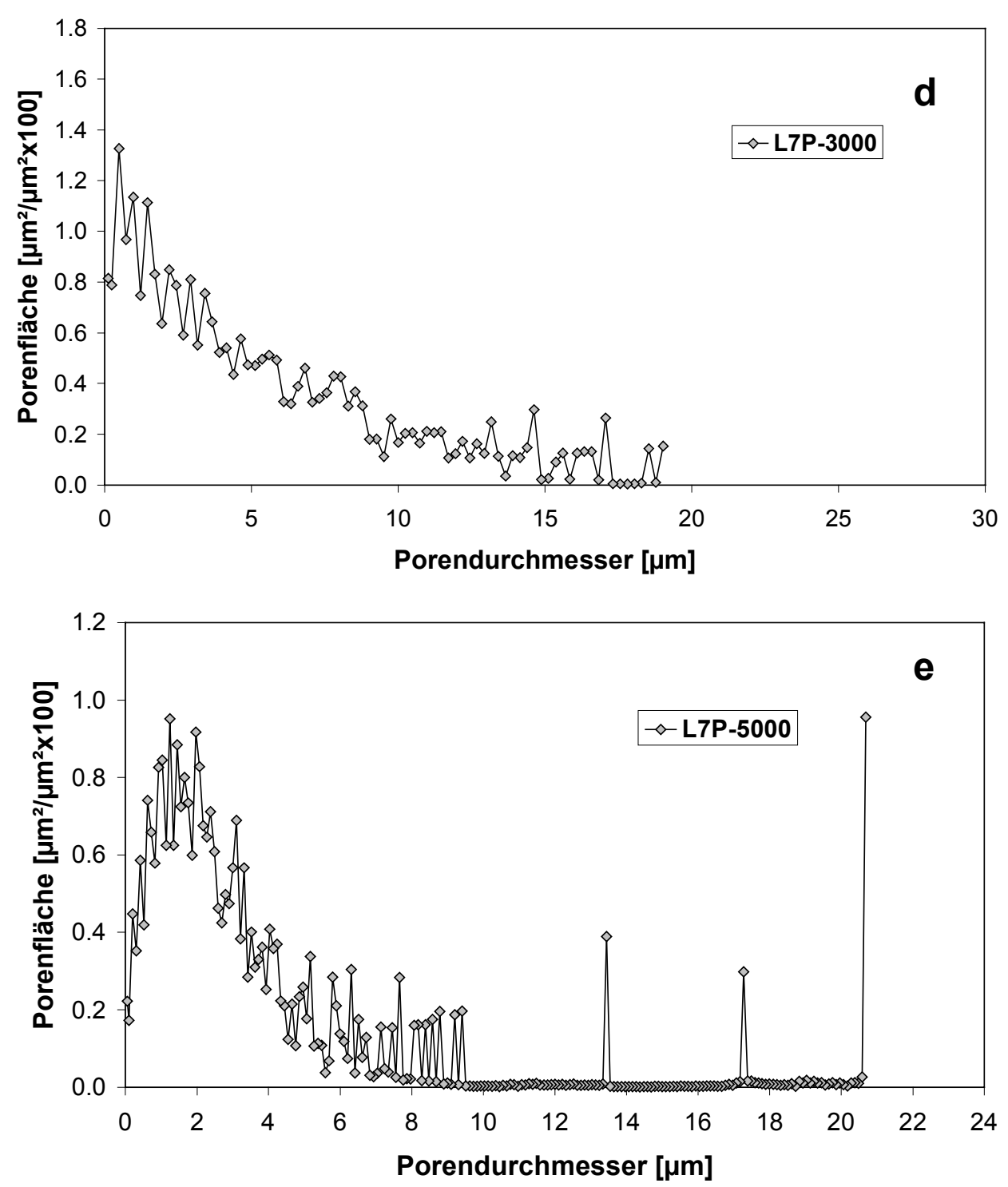

Abb. 6.17: Distributive Porengrößenverteilung der Serie Leo7. Dargestellt sind die Mittelwerte der Porenflächenhäufigkeiten von je 19 bis 21 Bildern pro Auflösungsstufe und Durchmesser des skalierenden Elementes. Angaben zu den Datenbereichen sind in Tab. A.1 aufgelistet.

Werden die distributiven Porengrößenverteilungen der Dünnschliffe Leo7 (vertikal) und Leo8 (horizontal) miteinander verglichen fällt auf, dass die Verteilungen von Leo8 für die drei niedrigsten Vergrößerungsstufen (Abb. A.12 a, b, c) eine geringere Häufigkeit der kleinen Poren als die von Leo7 (Abb. 6.17 a, b, c) aufweisen. Die hohen Vergrößerungsstufen zeigen diesen Trend nicht so deutlich (s. jeweils $d$ und $e$ in den Abb. 6.17 und A.12).

\subsubsection{Vergleich der kumulierten Porengrößenverteilung mit der Retentionskurve}

Der Vergleich von bildanalytisch gemessenen Porengrößenverteilungen als Summenkurve mit der im Labor erstellten Retentionskurve erbringt für beide Bildserien erstaunlich gute Übereinstimmungen vor allem im Bereich geringer Wasserspannungen (Abb. 6.18 \& 6.19). Dort übersteigt die kumulierte Porenfläche den in Volumenprozent angegebenen 
Wassergehalt. Für hohe Saugspannungen dagegen verläuft die Summenkurve der Porengrößen deutlich im Bereich geringerer Porenanteile als der Äquivalentporendurchmesser der Retentionskurve vermuten lässt. Die Überschneidung der beiden Kurven liegt bei Leo7 zwischen 260 und $280 \mathrm{hPa}$ bzw. 11,54 und 10,71 $\mu \mathrm{m}$ Äquivalentporendurchmesser (s. Abb. 6.18). Die kumulierten Porenflächen der Serie Leo8 schneiden die Retentionskurve deutlich früher: Der Schnittpunkt liegt zwischen 70 und 100 hPa bzw. 42,86 und 30,00 $\mu \mathrm{m}$ Porendurchmesser. Die parallel verschobenen Kurven aller Vergrößerungsstufen, ihre Aufsummierung und die Darstellung ohne die ersten fünf Messwerte bis zu der Porengrößenklasse, die auf mindestens $50 \%$ aller Bilder vorkommt findet sich für Leo8 im Anhang als Abb. A.13.

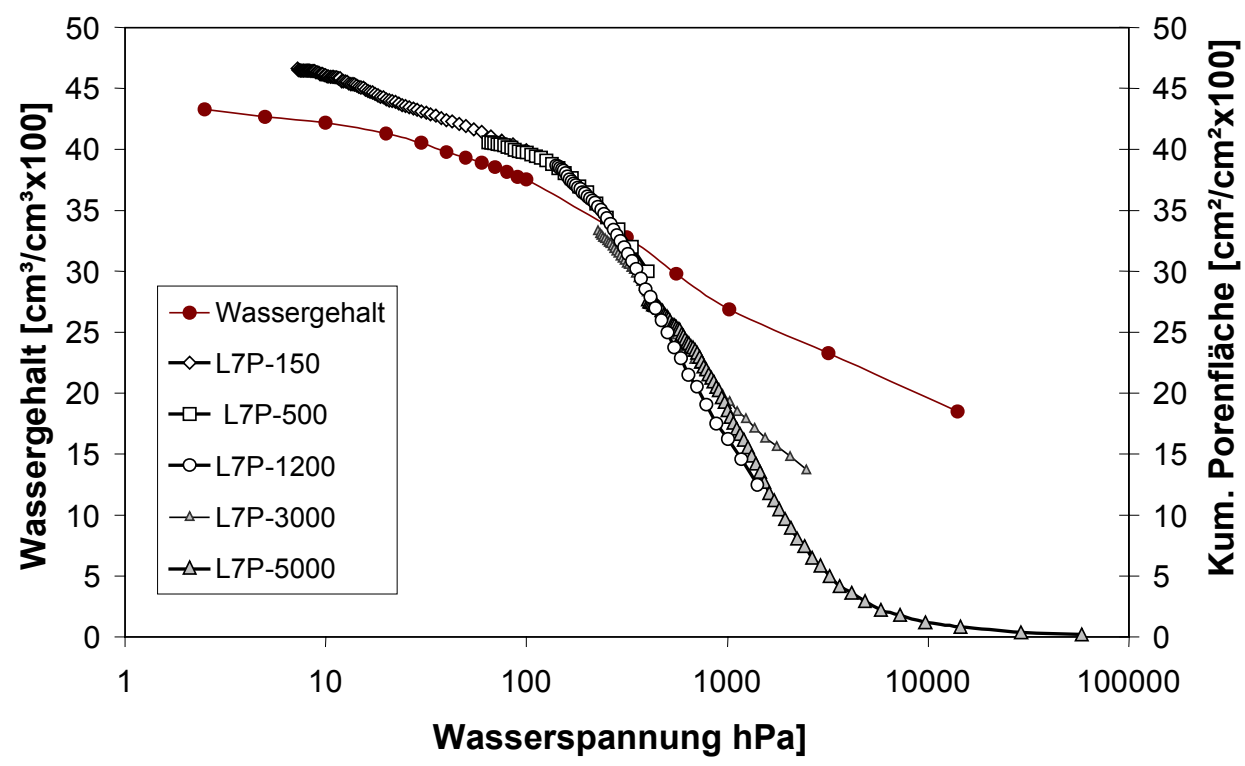

Abb. 6.18: Vergleich der Porengrößenverteilungen für Leo7 mit der am selben Bodenhorizont aufgenommenen Retentionskurve. Die Verteilung für die kleinsten Poren der höchsten und die größten Poren der niedrigsten Vergrößerungsstufe sind zusätzlich zu den in $(5.10 \mathrm{c})$ gezeigten Daten abgebildet.

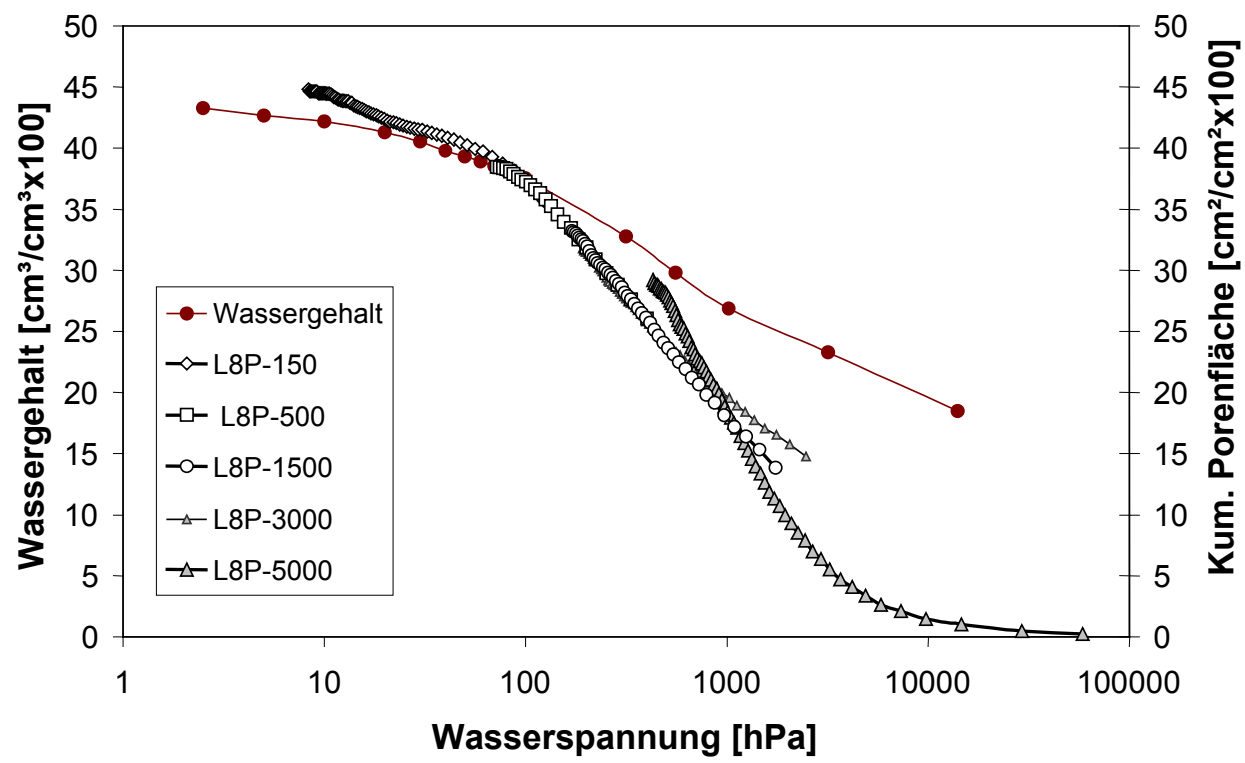

Abb. 6.19: Vergleich der Porengrößenverteilungen für Leo8 mit der am selben Bodenhorizont aufgenommenen Retentionskurve. Die Verteilung für die kleinsten Poren der höchsten und die größten Poren der niedrigsten Vergrößerungsstufe sind zusätzlich zu den in (A.13 c) gezeigten Daten abgebildet. 
Der höchste hier gezeigte Wassergehalt beträgt 43,29 Vol \% bei einer Saugspannung von 2,5 hPa. Nach der Kapillarengleichung entspricht dies einem Porendurchmesser von 1,20 mm. Die größten mit dem FE-REM erfassten Poren haben einen Durchmesser von $415 \mu \mathrm{m}$ (Serie Leo7, s. Abb. 6.18) und $361 \mu \mathrm{m}$ für die Serie Leo8 (s. Abb. 6.19). Wird die Porenfläche der lichtmikroskopischen Aufnahmen der für Leo7 und 8 verwendeten Dünnschliffe ab diesem Bereich addiert, kommen für Leo7 0,36 \% und für Leo8 1,19\% Porenfläche hinzu (nicht abgebildet). Damit liegt die maximal erfasste Porengröße bei 598 $\mu \mathrm{m}$ (Leo7) bzw $621 \mu \mathrm{m}$ (Leo8), was zu einem Maximum der kumulierten Porenfläche von 47,00 bzw. 45,98 \% führt. Die den größten lichtmikroskopisch erfassten Porendurchmessern entsprechenden Wasserspannungen betragen 5,08 und 4,83 hPa.

\subsubsection{Darstellung der Porengrößenverteilung aller Vergrößerungsstufen}

Wie in Kapitel 5.3 beschrieben, können die Porengrößenverteilungen verschiedener Vergrößerungsstufen, also unterschiedlicher Klassenbreiten, in einer Abbildung dargestellt werden wenn durch die jeweilige Klassenbreite geteilt wird. Die Klassenbreite ist hier gleichbedeutend mit der Schrittweite des skalierenden Elementes, die zwei Pixel betrug und somit der Auflösung proportional ist. Die Darstellung erfolgt logarithmisch, da dann auch die geringen Häufigkeiten (wie die Daten nahe der x-Achse in Abb. 6.17 a,b,c und e) deutlich erkennbar sind.

Um auch die größten Poren erfassen zu können, ist die Häufigkeitsverteilung der Poren mit einem Durchmesser $>85 \mu \mathrm{m}$ (Leo7, s. Abb. 6.20) und $>87,81 \mu \mathrm{m}$ (Leo8, s. Abb. 6.21) anhand der mit dem Wild-Photomakroskop erstellten Aufnahmen ebenfalls abgebildet. Für die an Hand der REM Aufnahmen gewonnenen Daten ist für jede Vergrößerungsstufe der Porengrößenbereich von den kleinsten erfassten Poren an bis zu dem Porendurchmesser dargestellt, an dem der Variationskoeffizient $\mathrm{cv}<100 \%$ wurde. Von daher ist im Gegensatz zu den Abb. 6.18 und 6.19 in Richtung großer Poren ein jeweils kleinerer Bereich und in Richtung kleiner Poren sind alle Messwerte jeder Vergrößerungsstufe dargestellt.

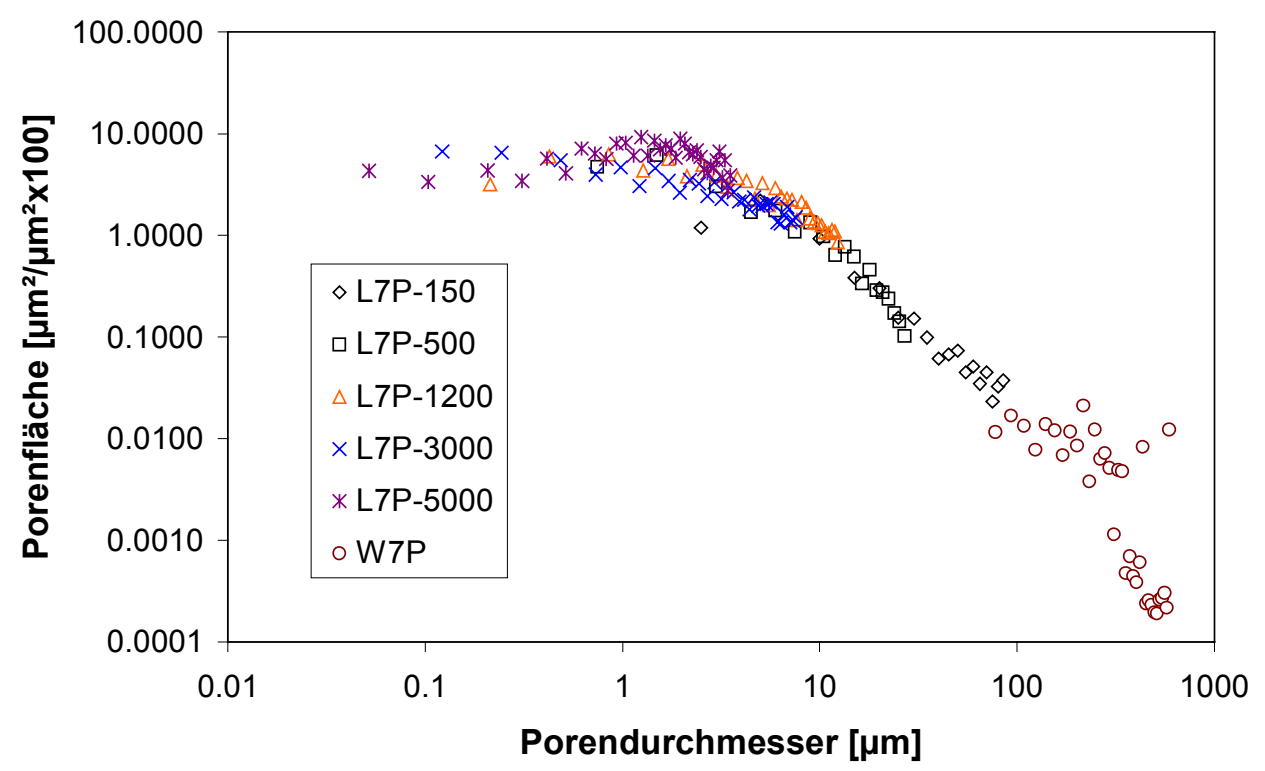

Abb. 6.20: Klassennormierte Porengrößenverteilung aller Vergrößerungsstufen für den Dünnschliff Leo7. Die lichtmikroskopisch ermittelten großen Poren desselben Schliffes (W7P) sind ebenfalls dargestellt. 
Auffällig ist, dass die Porengrößenverteilung ein Maximum bei 1,24 $\mu \mathrm{m}$ Porendurchmesser zeigt, wenn die Maxima für Poren $<0,50 \mu \mathrm{m}$ für Leo8 (Abb. 6.21) nicht berücksichtigt werden. In Richtung großer Durchmesser nehmen die Häufigkeiten ab. Erst im lichtmikroskopisch erfassten Größenbereich deutet sich ein sekundäres Maximum zwischen 200 und $600 \mu \mathrm{m}$ Porendurchmesser an (s. die Serien W7P und W8P in den Abb. 6.20 und 6.21).

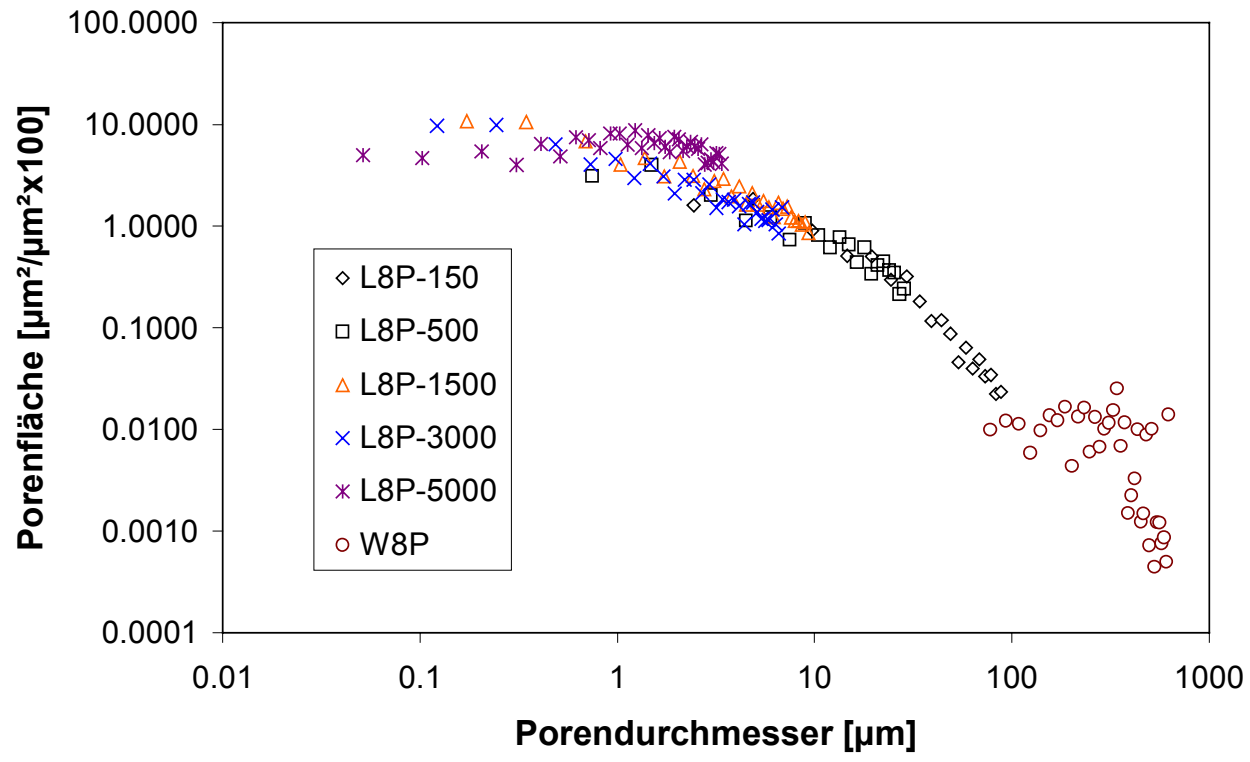

Abb. 6.21: Klassennormierte Porengrößenverteilung aller Vergrößerungsstufen für den Dünnschliff Leo8. Die lichtmikroskopisch ermittelten großen Poren desselben Schliffes (W8P) sind ebenfalls dargestellt.

\subsubsection{Messungen an lichtmikroskopischen Aufnahmen}

In den Abb. 6.22 und 6.23 sind die Porenflächenhäufigkeiten der lichtmikroskopisch aufgenommenen Serien WK2hor und WK2ver dargestellt. Auch hier zeigt sich ein Maximum für kleine Porendurchmesser, was bei den horizontal ausgerichteten Schliffen ausgeprägter ist. Es sind jeweils die mit dem Opening-Verfahren erzielten Ergebnisse von den kleinsten erfassten Poren an bis zu dem Porendurchmesser, der auf mindestens $50 \%$ aller Bilder vorkommt, dargestellt. 


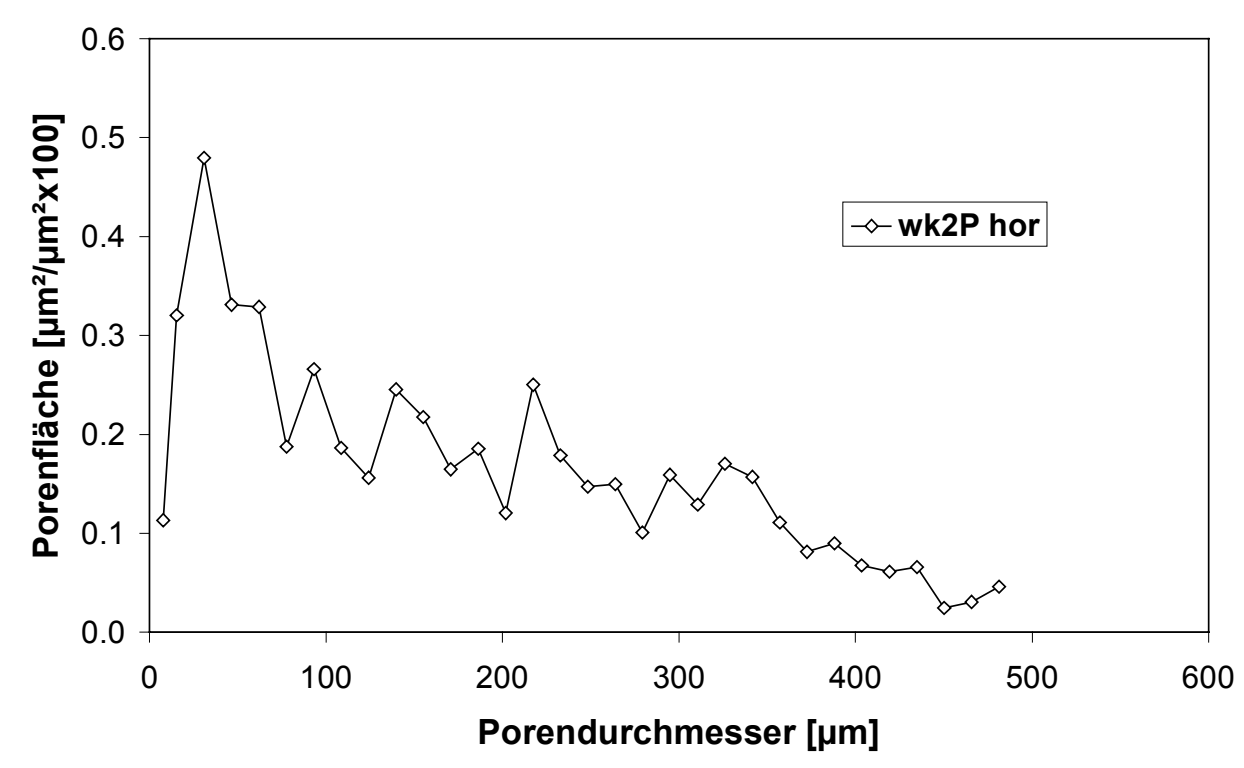

Abb. 6.22: Distributive Porengrößenverteilung der Serie WK2P hor. Dargestellt sind die Mittelwerte der Porenflächenhäufigkeiten von 7 Bildern bezogen auf den Durchmesser des skalierenden Elementes $(15,53 \mu \mathrm{m})$. Die Daten sind von den kleinsten Poren mit der Größe eines Pixels bis zu der Porengröße dargestellt, die auf mindestens $50 \%$ aller Bilder vorkommt.

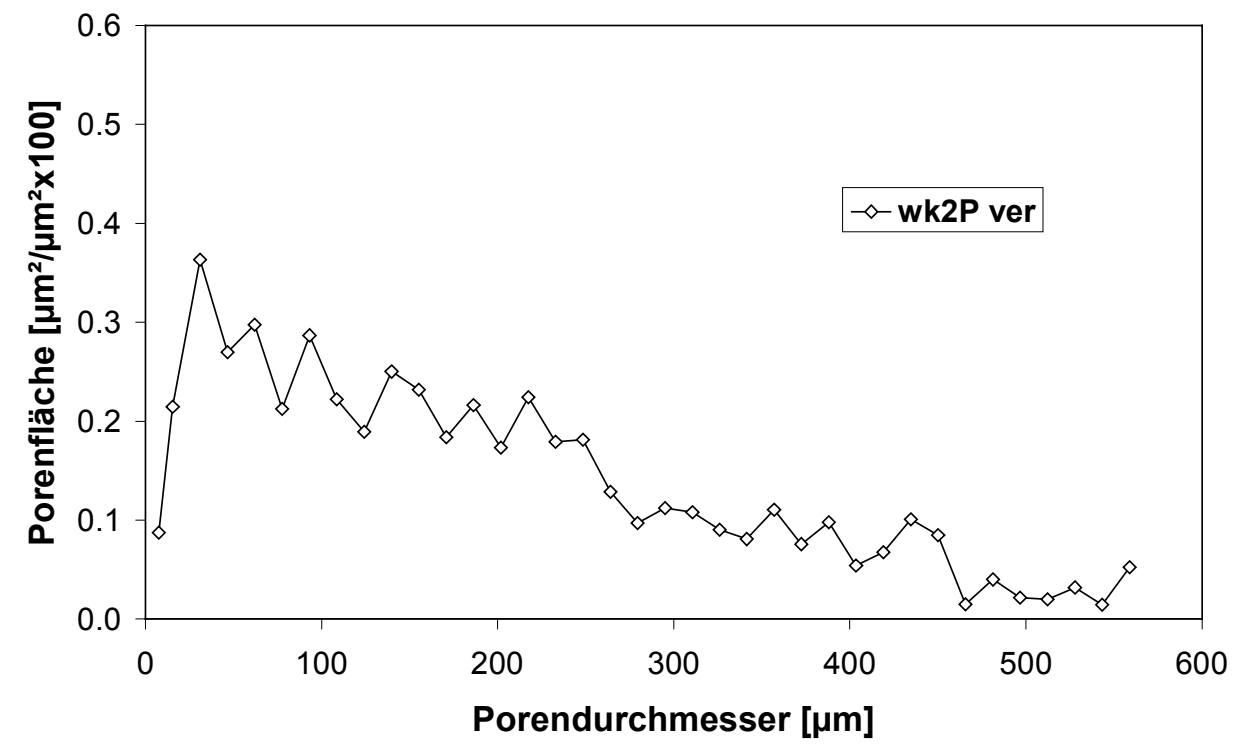

Abb. 6.23: Distributive Porengrößenverteilung der Serie WK2P ver. Dargestellt sind die Mittelwerte der Porenflächenhäufigkeiten von 10 Bildern bezogen auf den Durchmesser des skalierenden Elementes $(15,53 \mu \mathrm{m})$. Die Daten sind von den kleinsten Poren mit der Größe eines Pixels bis zu der Porengröße dargestellt, die auf mindestens $50 \%$ aller Bilder vorkommt.

Die Summe der auf diese Weise erfassten Poren beträgt für die horizontal ausgerichteten Dünnschliffe 5,14 \% der gesamten Bildfläche für Porendurchmesser von 7,76 bis 483,31 $\mu \mathrm{m}$ und für die horizontal ausgerichteten Dünnschliffe 5,30 \% für die Durchmesser von 7,76 bis $558,94 \mu \mathrm{m}$. 


\subsection{Die Steigung der Ableitung der Porengrößenverteilung}

Um eine bzw. zwei Geraden für die in Kapitel 6.3.3 dargestellten distributiven Porengrößenverteilung der Serien Leo7 und Leo8 anpassen zu können, wurde das in Kapitel 5.1.5 beschriebene robuste Schätzverfahren verwendet. Es handelt sich um die in Abb. 6.20 und 6.21 gezeigten Daten, wobei nur die mit dem REM Aufnahmen erhaltenen Messergebnisse verwendet wurden. Die Ergebnisse für eine Gerade sind in Abb. 6.24 für Leo7 und in Abb. 6.25 für Leo8 dargestellt, für zwei Geraden in den Abb. 6.26 b und c (Leo7) und $6.27 \mathrm{~b}$ und c (Leo8). In der Tab. 6.16 sind alle Ergebnisse zusammengestellt. Dort werden die negativen Steigungen der Geraden wie fraktale Dimensionen behandelt. Die Abb. 6.24 bis 6.25 skalieren auf der x-Achse umgekehrt zu den Abb. 6.18 und 6.19. So werden in dem hier gezeigten Bereich die Häufigkeiten der kleinen Porendurchmesser viel deutlicher abgebildet als in den Abb. 6.18 und 6.19. Dort ist auf der y-Achse die kumulative Porenhäufigkeit aufgetragen, nur die x-Achse skaliert logarithmisch. Dadurch ist der Bereich der Wasserspannung, der großen Poren zuzuordnen ist, deutlicher erkennbar.

Für die Serie Leo8 erwies sich die Anpassung einer Geraden als die beste, die absolute Abweichung von 0,193 wurde durch die Anpassung von zwei Geraden nicht verbessert. Für zwei Geraden ergaben sich pro Messserie zwei lokale Minima der absoluten Abweichungen (s. Abb. 6.26 a und 6.27 a), für die die Geradengleichungen in den Abbildungen angegeben sind (jeweils unter $b$ und $c$ ) und die Schnittpunkte der Geraden berechnet wurden (s. Tab. 6.16).

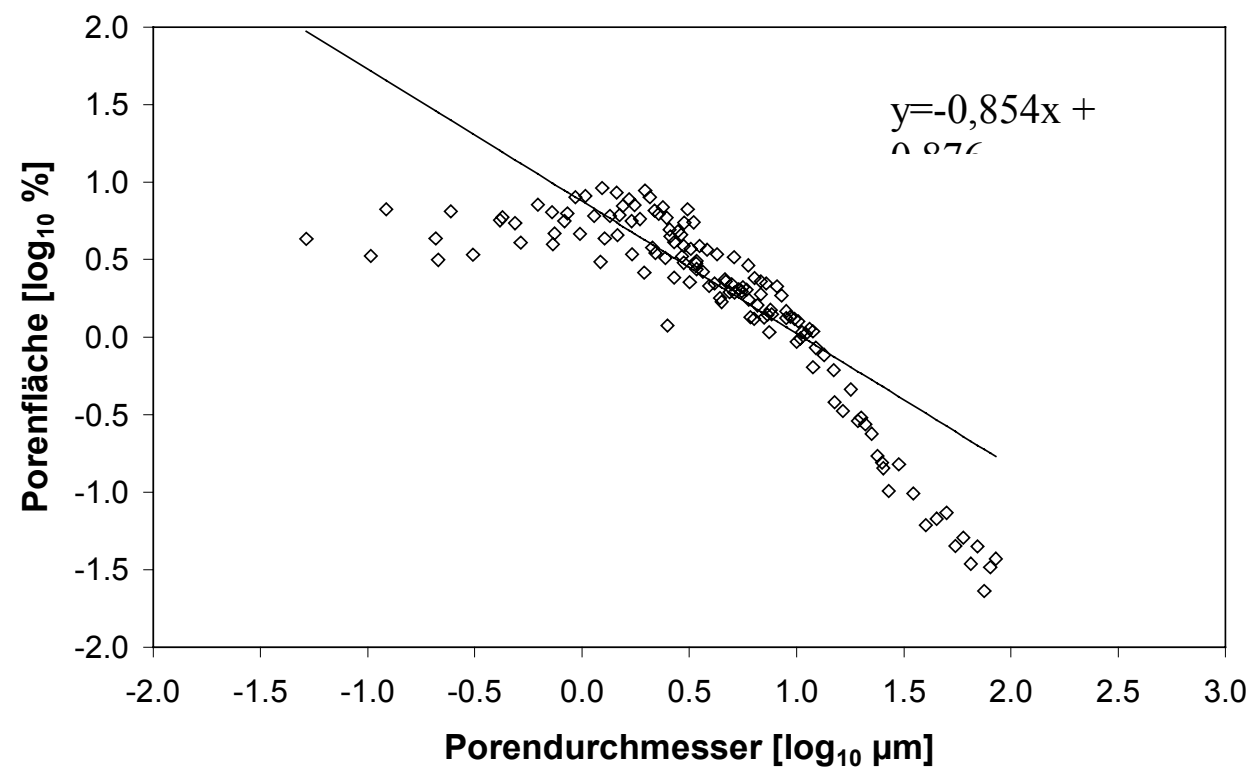

Abb. 6.24: Anpassung von einer Geraden mit dem robusten Schätzverfahren an die logarithmisch dargestellte distributive Porengrößenverteilung von Leo7. Die Geradengleichung ist angegeben. 


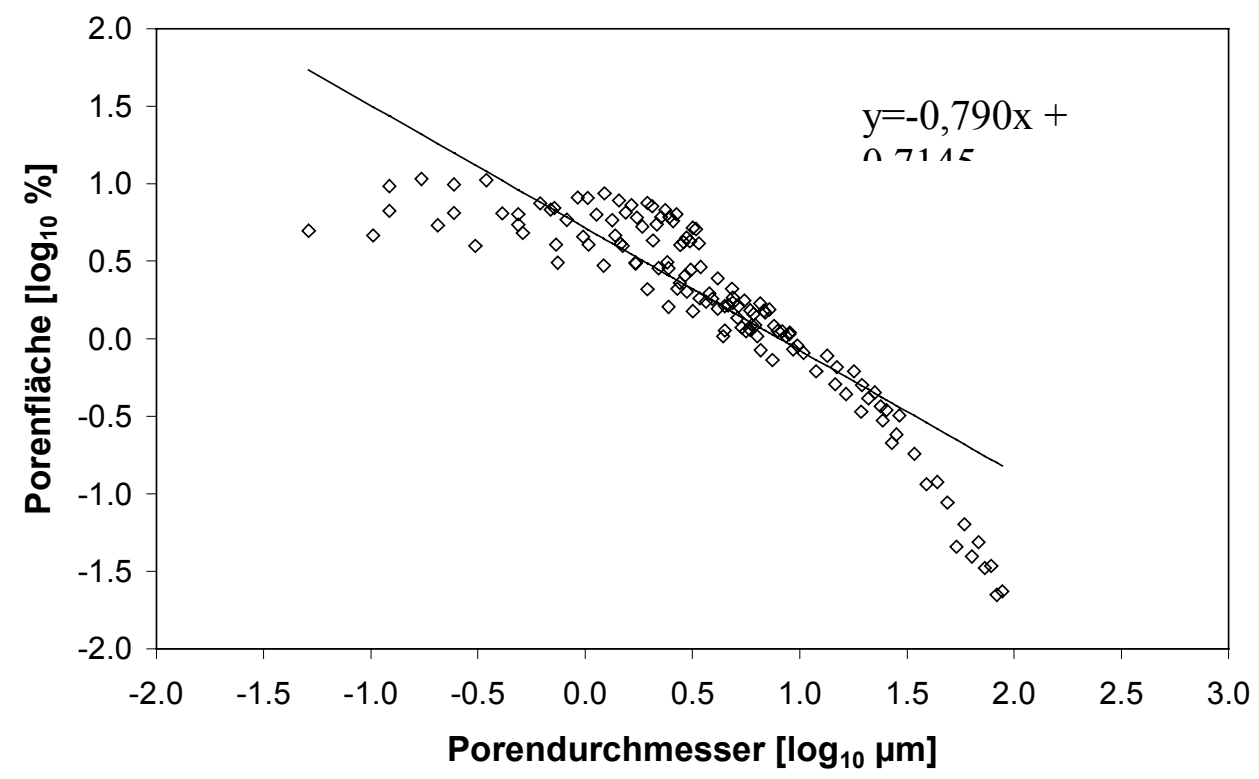

Abb. 6.25: Anpassung von einer Geraden mit dem robusten Schätzverfahren an die logarithmisch dargestellte distributive Porengrößenverteilung von Leo8. Die Geradengleichung ist angegeben.

Einfache Skalierungsgleichungen mit einem fraktalen Exponenten D für die Abhängigkeit einer Anzahl oder Häufigkeit (von Körnern, Poren etc.) von ihrem Durchmesser liegen nach Kenntnisstand der Autorin lediglich für kumulierte Verteilungen vor. Daher ist hier von Steigungen und nicht von Dimensionen die Rede, auch wenn die in Tabelle 6.16 angegebenen Steigungen der logarithmierten distributiven Porengrößenverteilung mit $D^{P}$ abgekürzt werden. Man sollte sich jedoch der Tatsache bewusst sein, dass die kumulierte Verteilung das Integral der distributiven Verteilung über die Größe der skalierenden Elemente darstellt. 

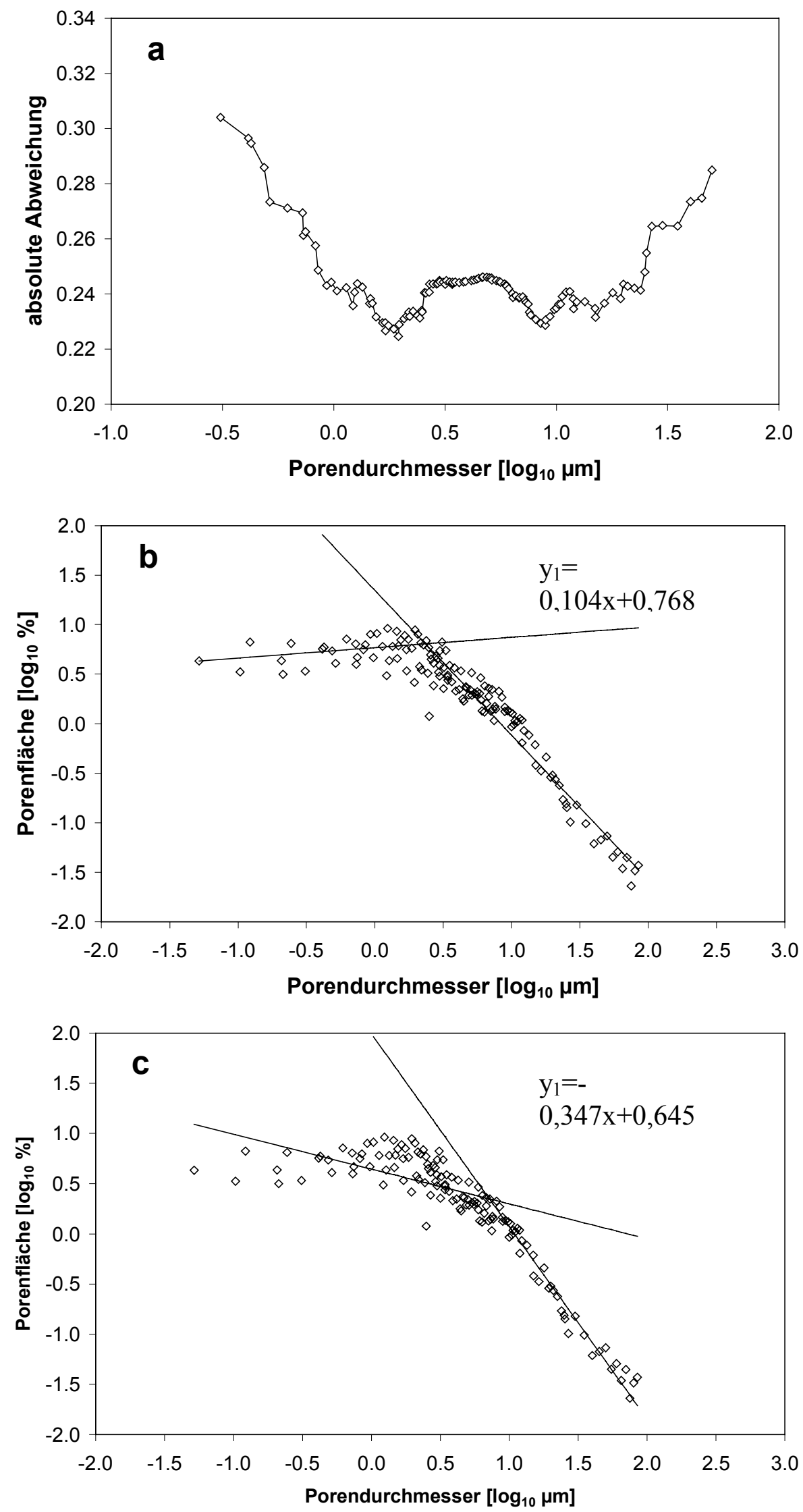

Abb. 6.26: Anpassung von zwei Geraden an die logarithmisch dargestellte distributive Porengrößenverteilung von Leo7. (a) Die absoluten Abweichungen weisen zwei Maxima auf; (b) Anpassung von zwei Geraden für das erste Maximum und (c) für das zweite Maximum. Die Geradengleichungen sind jeweils angegeben. 

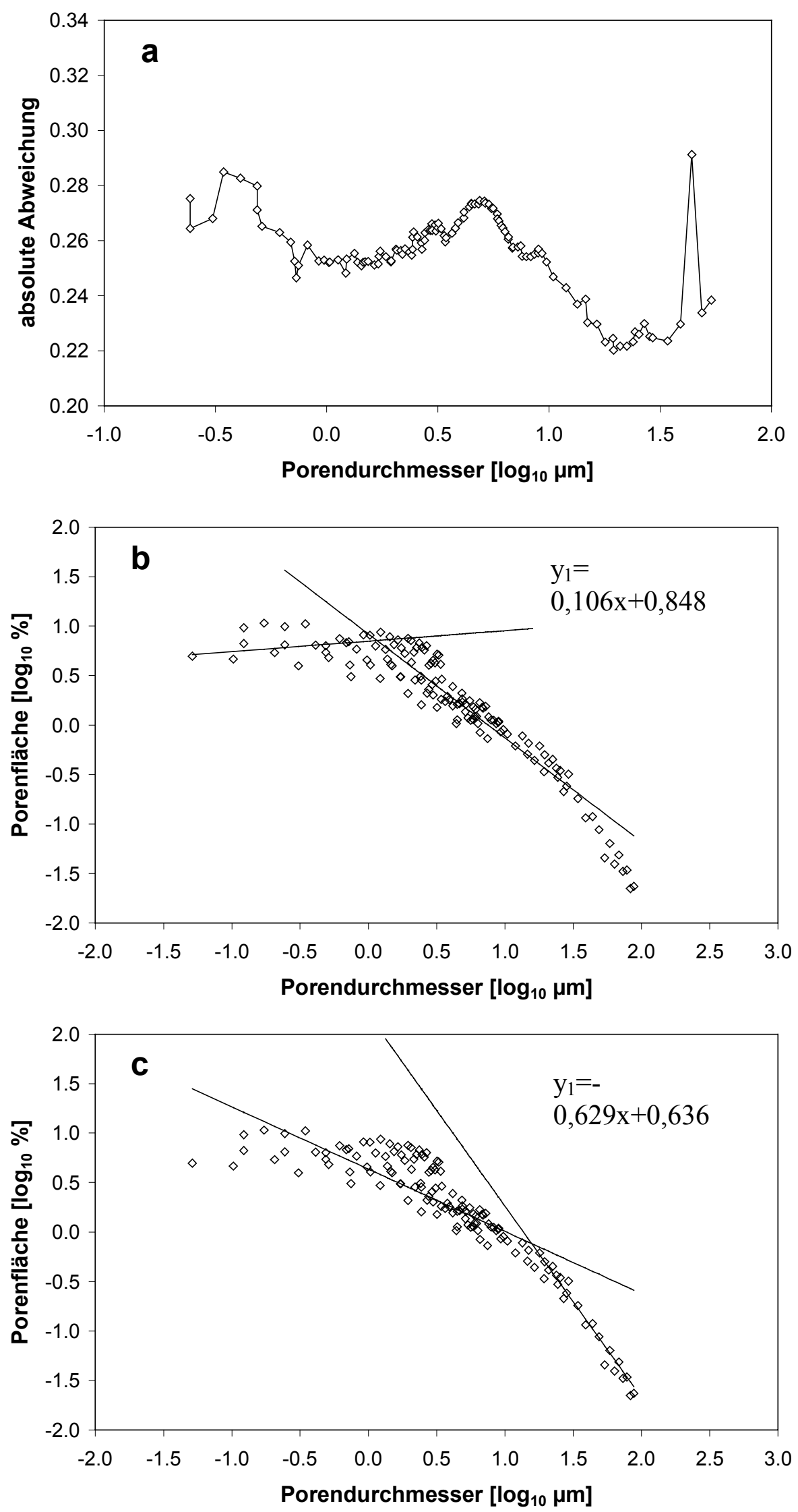

Abb. 6.27: Anpassung von zwei Geraden an die logarithmisch dargestellte distributive Porengrößenverteilung von Leo8. (a) Die absoluten Abweichungen weisen zwei Maxima auf; (b) Anpassung von zwei Geraden für das erste Maximum und (c) für das zweite Maximum. Die Geradengleichungen sind jeweils angegeben. 
Eine fraktal skalierende Porengrößenverteilung ist in beiden Fällen nur in den Bereichen gegeben, in denen die Steigung der Verteilung über mindestens eine, möglichst zwei oder mehr Größenordnungen konstant bleibt. Diese Steigung verläuft für die distributive Porengrößenverteilung von Leo7 mit $-0,854$ (s. Abb. 6.24) steiler als für Leo8 mit $-0,790$ (s. Abb. 6.25 und jeweils Tab. 6.16). Für die kumulierten Verteilungen (vergleiche Abb. 6.18 und 6.19) gilt das Gleiche. Auch die Porengrößen, zwischen denen ein linearer Zusammenhang zwischen dem logarithmierten Porendurchmesser und dem distributiven (logarithmierten) bzw. kumulierten (nicht logarithmierten) Porenflächenanteil besteht, stimmen gut überein: Während der abfallende Ast der kumulierten Porenfläche von Leo7 (Abb. 6.18) bei ca. $220 \mathrm{hPa}$ Wasserspannung bzw. 13,64 $\mu \mathrm{m}$ Porendurchmesser beginnt, endet der lineare Bereich in Abb. 6.24 bei 13,43 $\mu \mathrm{m}$. Die kumulierten Porengrößenverteilungen skalieren bis rund $2636 \mathrm{hPa}$ bzw. 1,14 $\mu \mathrm{m}$ linear (Abb. 6.18), für die distributive Porengrößenverteilung (Abb. 6.24) erhält man denselben Wert.

Der abfallende Ast der kumulierten Porengrößenverteilung von Leo8 (Abb. 6.19) beginnt bei rund $112 \mathrm{hPa}$ Wasserspannung bzw. 26,79 $\mu \mathrm{m}$ Porendurchmesser, was gut mit dem Ende des linearen Bereichs in Abb. 6.25 übereinstimmt. Im Bereich kleiner Poren können für die kumulierte Verteilung $3250 \mathrm{hPa}$ bzw. 0,92 $\mu \mathrm{m}$ Porendurchmesser angegeben werden, was sehr gut mit dem Wert von $0,62 \mu \mathrm{m}$ in Abb. 6.25 übereinstimmt, wo die Anpassungsgerade den ersten Messpunkt schneidet.

Tab. 6.16: Steigungen $\left(D^{\mathrm{P}}\right)$ der distributiven Porengrößenverteilung der Serien Leo7 und Leo8, gemessen mit dem Opening-Verfahren. Die für jede Vergrößerungsstufe gemittelte Porenfläche pro Bildfläche in Prozent wurde auf die jeweilige Schrittweite des skalierenden Elementes bezogen (s. Kapitel 5.3). Die als Optimierungskriterium benutzten absoluten Abweichungen stehen in Klammern. $\mathrm{D}_{\mathrm{T}}$ ist die Steigung über den gesamten Vergrößerungsbereich, $\mathrm{D}_{1}$ wird der Textur im Bereich kleiner Skalierungselemente, $\mathrm{D}_{2}$ der Struktur im Bereich großer Skalierungselemente zugeordnet. Die hinter $D_{2}$ dargestellte absolute Abweichung bezieht sich auf $D_{1}$ und $D_{2}$.

\begin{tabular}{cccccc}
\hline Messserie & $\mathrm{D}_{\mathrm{T}}^{\mathrm{P}}$ & $\mathrm{D}_{1}^{\mathrm{P}}$ & $\mathrm{D}_{2}^{\mathrm{P}}$ & $\begin{array}{c}\text { Schnittpunkt } \\
{[\mu \mathrm{m}]}\end{array}$ \\
\hline \multirow{3}{*}{ Leo7 } & $0,854(0,240)$ & & & & - \\
\cline { 2 - 6 } & & $-0,104$ & 1,463 & $(0,225)$ & 2,343 \\
\hline \multirow{2}{*}{ Leo8 } & 1,463 & 1,919 & $(0,229)$ & 7,186 \\
\cline { 2 - 6 } & & & & & - \\
\cline { 2 - 6 } & & $-0,790(0,193)$ & 1,050 & $(0,246)$ & 1,156 \\
\hline
\end{tabular}

Werden zwei Geradengleichungen angepasst, erstrecken sich die Steigungen über Werte von $-0,106<\mathrm{D}^{\mathrm{P}}<1,934$. Dies zeigt die Schwierigkeit, die Werte als Dimensionen aufzufassen. Für die distributiven Verteilungen sind positive Steigungen möglich, so dass $\Delta \mathrm{D}^{\mathrm{P}}>2$ ist. 


\subsection{Ergebnisse der bodenphysikalischen Untersuchungen}

\subsubsection{Bestimmung der Phasenanteile}

In der Tabelle 6.17 sind die Ergebnisse der Messung der Phasenanteile dargestellt. Die Korngrößenverteilung zeigt mit 41,37 Gew. \% ein Maximum für Grobschluff $(20-63 \mu \mathrm{m}$ Durchmesser). Der Anteil der Sandfraktion $(63-2000 \mu \mathrm{m})$ beträgt 8,88 Gew. \%, der der Schlufffraktion $(2-63 \mu \mathrm{m}) 77,01$ Gew. \% und der der Tonfraktion $(<2 \mu \mathrm{m})$ 21,11 Gew. \%. Laut Bodenkundlicher Kartieranleitung (AG Boden 1994) ist der untersuchte Bodenhorizont als stark toniger Schluff (Ut4) anzusprechen.

Die Porosität wurde entsprechend Gl. 2.19 aus der Dichte der Festsubstanz und der Lagerungsdichte berechnet und beträgt 40,90 Vol. \%. Sie liegt damit unter dem höchsten gemessenen Wassergehalt (s. Abb. 6.29). Die Dichte der Festsubstanz ist mit 2,59 g/ $\mathrm{cm}^{3}$ etwas geringer als die von Quarz $\left(2,65 \mathrm{~g} / \mathrm{cm}^{3}\right)$.

Die in Tab. 6.17 angegebenen Eigenschaften sind für die Beschreibung der Bodenstruktur der untersuchten Proben von Bedeutung und können zu den Ergebnissen der digitalen Bildanalyse, insbesondere der Messung der fraktalen Dimension der Porenraumgrenzlinie und der Porengrößenverteilung, in Beziehung gebracht werden. Die Werte korrelieren gut mit denen aus publizierten Untersuchungen an tonigen Schluffhorizonten von Böden aus Löss in Südniedersachsen und Nordhessen (Rhodenburg \& Meyer 1979, Ahl et al. 1985).

Tab 6.17: Ergebnisse der Korngrößenanalyse (s. Tab. 2.1) und der Bestimmung der Phasenanteile. Die Porosität wurde aus der Dichte der Festsubstanz und der Lagerungsdichte berechnet.

\begin{tabular}{ccccc}
\hline Korngröße $[\mu \mathrm{m}]$ & $\begin{array}{c}\text { Massenanteil } \\
{\left[\mathrm{kg} \mathrm{kg}^{-1} \cdot 100\right]}\end{array}$ & $\begin{array}{c}\text { Lagerungs- } \\
\text { dichte }\left[\mathrm{g} \mathrm{cm}^{-3}\right]\end{array}$ & $\begin{array}{c}\text { Dichte der } \\
\text { Festsubstanz } \\
{\left[\mathrm{g} \mathrm{cm}^{-3}\right]}\end{array}$ & $\begin{array}{c}\text { Porosität } \\
{\left[\mathrm{cm}^{3} \mathrm{~cm}^{-3} \cdot 100\right]}\end{array}$ \\
\hline$>2000$ & 0,00 & & & \\
\hline $630-2000$ & 0,18 & & & \\
\hline $200-630$ & 3,16 & & & 40,90 \\
\hline $63-200$ & 5,54 & \multirow{2}{*}{1,53} & & \\
\hline $20-63$ & 41,37 & & & \\
\hline $6,3-20$ & 22,04 & & & \\
\hline $2,0-6,3$ & 6,60 & & & \\
\hline$<2,0$ & 21,11 & & & \\
\hline
\end{tabular}




\subsubsection{Messung der gesättigten und der ungesättigten Wasserleitfähigkeit}

Für die vertikal entnommenen Proben (16 Einzelproben) ergab sich eine mittlere gesättigte Wasserleitfähigkeit von $1,94 \mathrm{~cm} / \mathrm{d}$ mit einer Standardabweichung von $\pm 1,83 \mathrm{~cm} / \mathrm{d}$. Nach der Bodenkundlichen Kartieranleitung (AG Boden 1994) ist der untersuchte Bodenhorizont somit als gering durchlässig (kf2) einzustufen. Die Leitfähigkeiten der horizontal entnommen Proben (10 Parallelproben) schwankten zwischen 1,40 und 1779 $\mathrm{cm} / \mathrm{d}$ und damit so stark, dass von einer Mittelwertbildung abgesehen wurde. Die Standardabweichung betrug $\pm 567,49 \mathrm{~cm} / \mathrm{d}$. Tendenziell weisen die horizontal entnommenen Proben eine höhere gesättigte Wasserleitfähigkeit auf als die vertikal entnommenen. Dies deckt sich mit Beobachtungen während der Probennahme: Der Boden weist eine ausgeprägte horizontale Schichtung von Mächtigkeiten einiger mm auf, was häufig dazu führte, dass der obere Stechzylinderinhalt abplatzte und die Probe verworfen werden musste.

Die Ergebnisse der Messung der ungesättigten Wasserleitfähigkeit sind in Abb. 6.28 für 8 Messreihen an $250 \mathrm{~cm}^{3}$ Stechzylinderproben dargestellt. Die gemessenen Werte liegen zwischen $2,92 \mathrm{~cm} / \mathrm{d}$ für $25,60 \mathrm{~cm}$ Wassersäule (WS), bzw. $25,11 \mathrm{hPa}$ und $6,31 \cdot 10^{-4} \mathrm{~cm}$ WS bei $629,75 \mathrm{~cm}$ WS $(613,86 \mathrm{hPa})$. Der begrenzte Messbereich der Tensiometer ist deutlich zu erkennen, über $800 \mathrm{~cm}$ WS liegt keine Messung mehr vor. Dadurch, dass sich bei der Verdunstungsmethode (Gl 4.4 und 4.5) der angegebene Wert für die Saugspannung im Boden als Differenz zwischen oberem und unterem Tensiometer bestimmt wird, liegen die in Abb. 6.28 gezeigten Werte deutlich unter $800 \mathrm{~cm}$ WS.

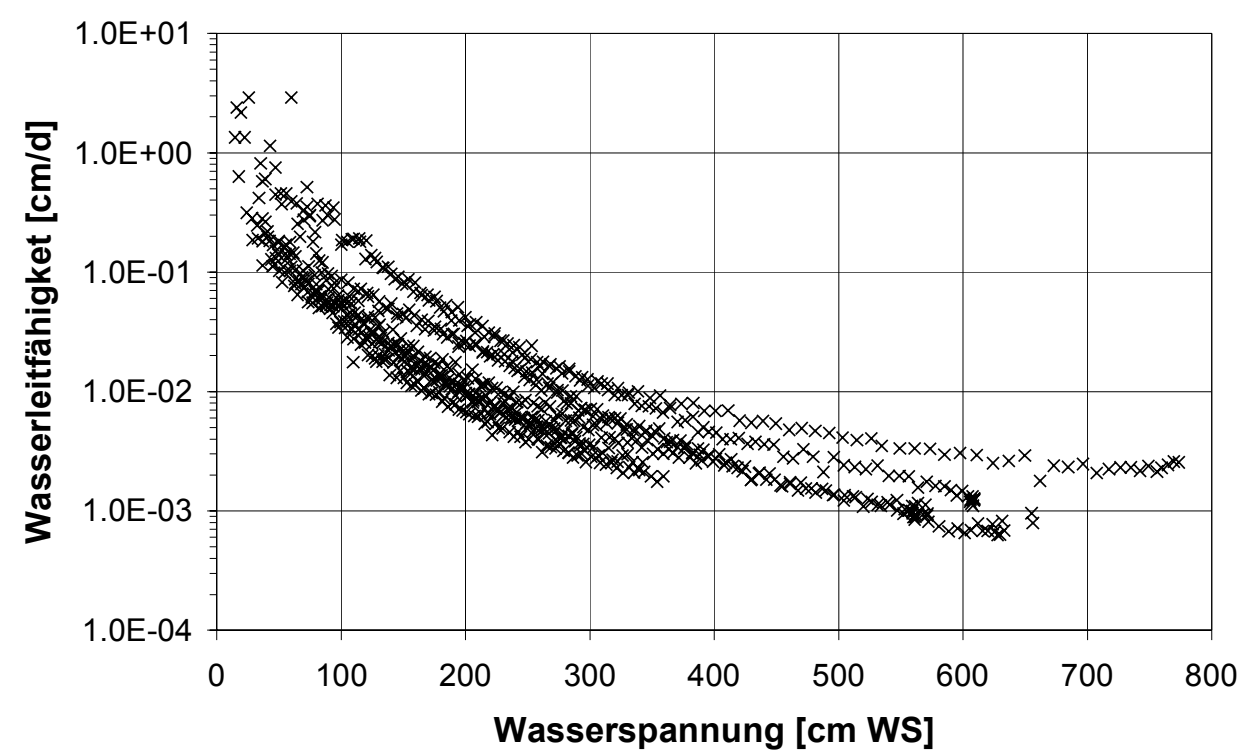

Abb. 6.28: Ungesättigte Wasserleitfähigkeiten von 8 an der Ku-Apparatur gemessenen Parallelproben. „WS“ bedeutet Wassersäule, $1 \mathrm{~cm} \mathrm{WS} \approx 0,981 \mathrm{hPa}$.

Bereits in diesem methodisch begrenzten Messbereich nimmt die hydraulische Leitfähigkeit um mehr als drei Größenordnungen ab. Die Differenz zur gesättigten Wasserleitfähigkeit $(1,94 \mathrm{~cm} / \mathrm{d})$ liegt innerhalb der Standardabweichung derselben und erklärt sich aus der relativ großen Schwankungsbreite bei der Messung der gesättigten 
Leitfähigkeit und dem zu Beginn des Verdunstungsprozesses geringem hydraulischen Gradienten.

\subsubsection{Schätzung der Parameter für die Retentionskurve}

Basierend auf den in Kapitel 2.2.1 beschriebenen mathematischen Formulierungen wurden die Parameter für die Retentionskurve (Wassergehalt als Funktion der Saugspannung) entsprechend den G1. 2.28 (Brooks \& Corey 1964) und 2.29 (van Genuchten 1980) angepasst. Dies erfolgte mit dem in Kapitel 5.4 kurz vorgestellten Programm RETC (van Genuchten et al. 1991). Die Ergebnisse sind in Tab. 6.18 und in den Abb. 6.29 und 6.30 dargestellt. Für die doppelte van Genuchten-Gleichung wurden zwei Parametersätze angepasst, der erste bis $100 \mathrm{hPa}(<\mathrm{pF} 2,0)$ und der zweite von 100 bis $14000 \mathrm{hPa}(\mathrm{pF} 2,0-$ $4,2)$. Der quadrierte Produktmomenten-Korrelationskoeffizient ist jeweils angegeben.

Tab. 6.18: Anpassungsparameter zur Beschreibung der unter statischen Gleichgewichtsbedingungen ermittelten Retentionskurve unter der Annahme eines unimodalen und eines bimodalen Porensystems (maximal 30 Iterationen), $\mathrm{r}^{2}=$ Korrelationskoeffizient. Erweiterert aus Friedrichs, 1999.

\begin{tabular}{|c|c|c|c|}
\hline \multirow{2}{*}{ Parameter } & \multirow{2}{*}{$\begin{array}{c}\text { einfache van } \\
\text { Genuchten-Gleichung }\end{array}$} & \multicolumn{2}{|c|}{ doppelte van Genuchten-Gleichung } \\
\hline & & $\begin{array}{l}\text { sättigungsnaher } \\
\text { Bereich }(<\mathrm{pF} 2,0)\end{array}$ & $\begin{array}{l}\text { sättigungsferner } \\
\text { Bereich (pF 2,0-4,2) }\end{array}$ \\
\hline$\theta_{\mathrm{s}}\left[\mathrm{cm}^{3} \mathrm{~cm}^{-3}\right]$ & 0,43333 & 0,43828 & 0,44393 \\
\hline$\theta_{\mathrm{r}}\left[\mathrm{cm}^{3} \mathrm{~cm}^{-3}\right]$ & 0,01992 & 0,00000 & 0,10082 \\
\hline$\alpha[1 / \mathrm{cm}]$ & 0,02039 & 0,07334 & 0,01576 \\
\hline $\mathrm{n}[1]$ & 1,16173 & 1,07260 & 1,25352 \\
\hline $\mathrm{m}[1]$ & 0,13921 & 0,06769 & 0,20225 \\
\hline$r^{2}$ & 0,9989 & \multicolumn{2}{|c|}{0,9997} \\
\hline & Brooks und Corey & & \\
\hline$\theta_{\mathrm{s}}\left[\mathrm{cm}^{3} \mathrm{~cm}^{-3}\right]$ & 0,427015 & & \\
\hline$\theta_{\mathrm{r}}\left[\mathrm{cm}^{3} \mathrm{~cm}^{-3}\right]$ & 0,00000 & & \\
\hline$\Psi_{\mathrm{b}}[\mathrm{cm}]$ & 27,62431 & & \\
\hline$\lambda[1]$ & 0,12412 & & \\
\hline$r^{2}$ & 0,9835 & & \\
\hline
\end{tabular}

Für die Anpassung nach van Genuchten korrelieren die Werte gut mit Angaben aus der Literatur (Carsel \& Parrish 1988, zitiert in van Genuchten et al. 1991, Kapitel 2) für tonigen Lehm bzw. schluffig tonigen Lehm. Eine Ausnahme bildet der sättigungsnahe Bereich bei der Anpassung von zwei Parametersätzen: Während $\alpha=0,07334$ auf einen sandigen Lehm hinweist, deutet $\mathrm{n}=1,07260$ auf einen schluffigen Ton hin. Der 
Parametersatz für den sättigungsfernen Bereich hingegen steht für einen schluffig tonigen Lehm als Bodenart. Die Parameter nach Brooks und Corey beschreiben einen recht frühen Lufteintrittspunkt mit $\psi_{\mathrm{b}} \approx 27,62 \mathrm{~cm}$ WS und mit $\lambda=0,12412$ eine sehr weite Porengrößenverteilung.

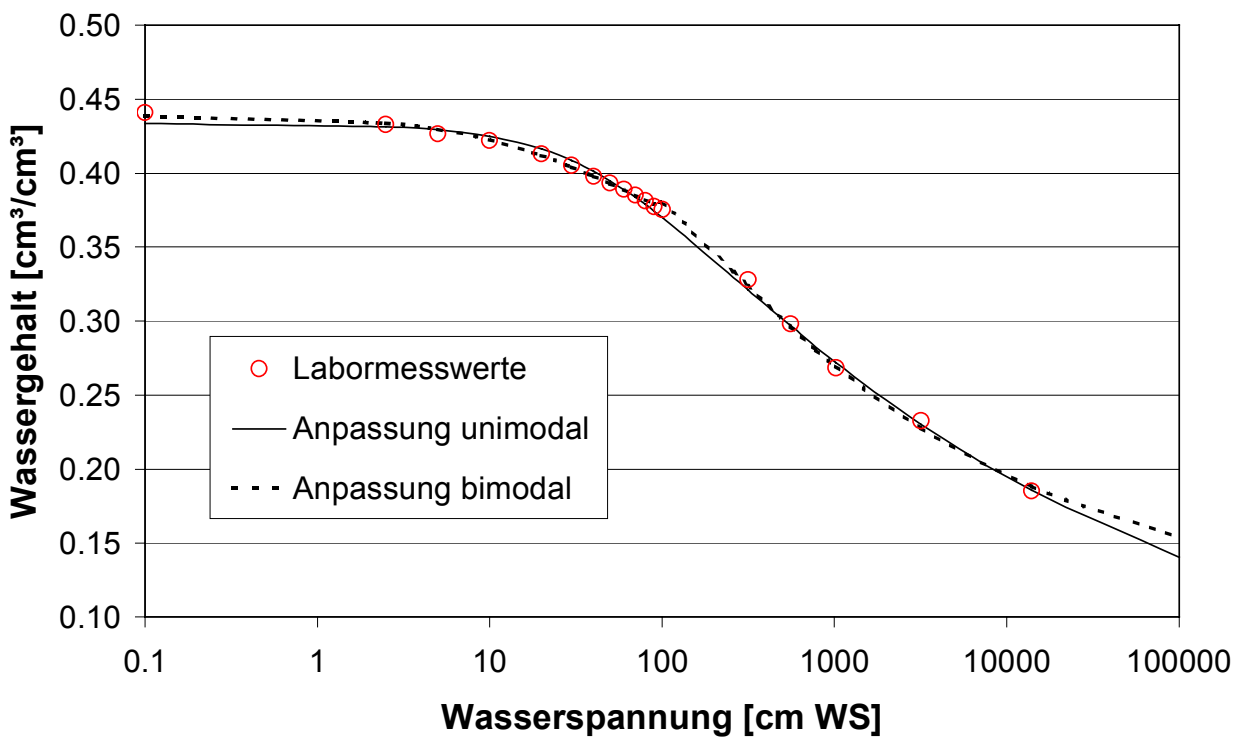

Abb. 6.29: Im Labor unter statischen Gleichgewichtsbedingungen gemessene Werte von Wassergehalt und Wasserspannung und die Anpassung von Retentionskurven nach van Genuchten. Es wurde ein unimodales (durchgezogene Kurve) und ein bimodales (gestrichelte Kurve) Porensystem angenommen. Die Anpassungsparameter sind in Tab. 6.18 angegeben.

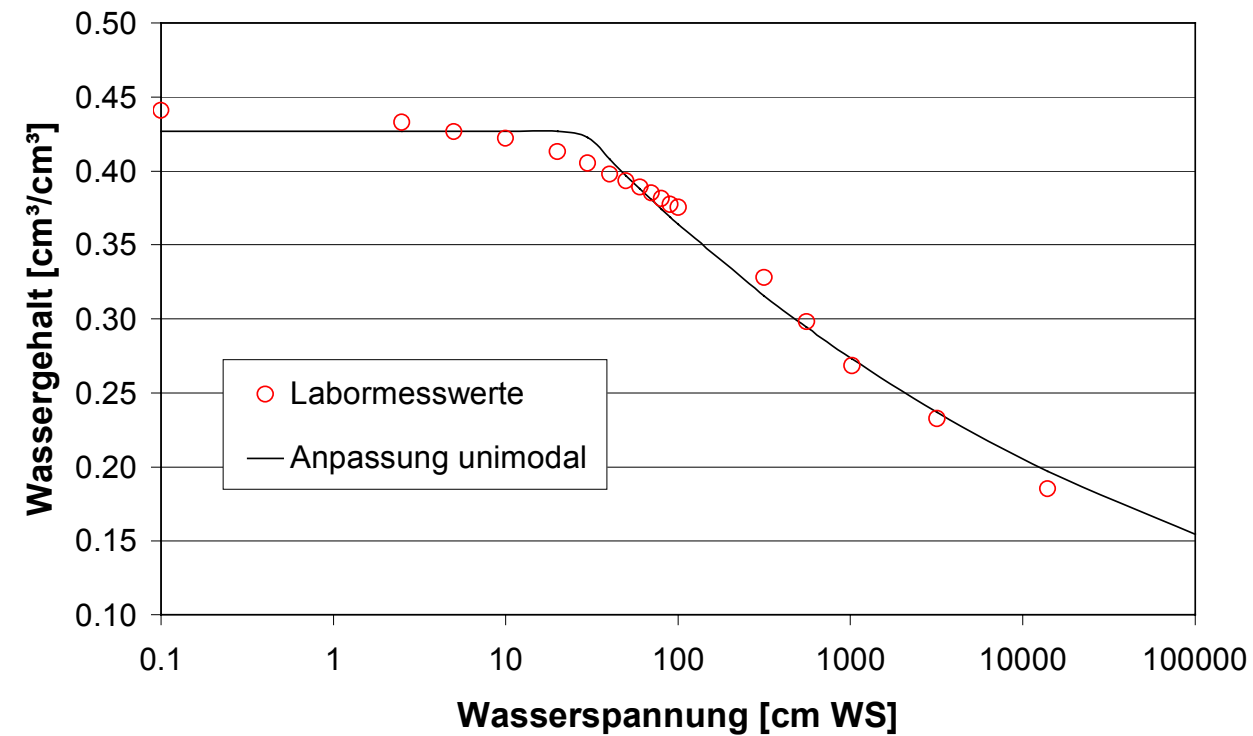

Abb. 6.30: Im Labor unter statischen Gleichgewichtsbedingungen gemessene Werte und die Anpassung einer Retentionskurve nach Brooks und Corey unter der Annahme eines unimodalen Porensystems. Die Anpassungsparameter sind in Tab. 6.18 angegeben. 


\subsubsection{Berechnung der ungesättigten Wasserleitfähigkeit und Vergleich mit den Messdaten}

Die Berechnung der ungesättigten Wasserleitfähigkeit erfolgte analog zu den Modellvorstellungen von Mualem (1976) aus den nach van Genuchten erhobenen Parametern. Unter der Annahme einer unimodalen Porengrößenverteilung wurde die Gl. 2.31 direkt benutzt, für die bimodale Porengrößenverteilung wurden die Leitfähigkeiten aus beiden Retetentionskurven über den gesamten betrachteten Wasserspannungsbereich summiert (Diekkrüger 1992, Kapitel 4). Dieses Vorgehen implementiert die Annahme, dass zwei Porensysteme vorhanden sind. Die Ergebnisse sind in Abb. 6.31 zusammen mit den gemessenen ungesättigten Leitfähigkeiten dargestellt.

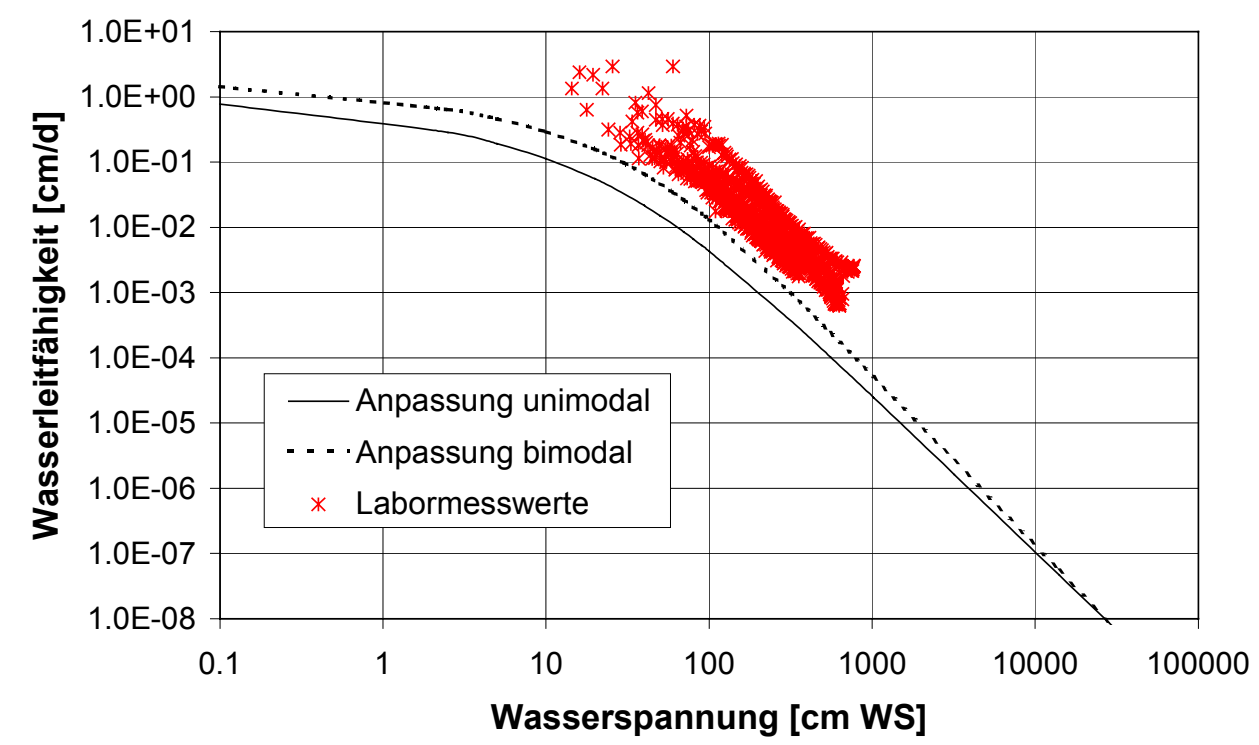

Abb. 6.31: Berechnung der ungesättigten Wasserleitfähigkeit nach den Modellvorstellungen von Mualem und van Genuchten unter der Annahme eines unimodalen (durchgezogene Kurve) und eines bimodalen (gestrichelte Kurve) Porensystems. Zum Vergleich sind die unter quasi-stationären Bedingungen gemessenen ungesättigten Wasserleitfähigkeiten dargestellt.

Beide Anpassungskurven erstrecken sich über rund 7 Größenordnungen und verlaufen in Richtung geringerer Leitfähigkeiten als die gemessenen Werte. Es ist eindeutig erkennbar, dass die unter der Annahme eines bimoden Porensystems angepasste Leitfähigkeitskurve die Messwerte besser wiedergibt. Durch die Anpassung von zwei Retentionskurven wird die Leitfähigkeit in dem Wasserspannungsbereich, der mit den Porendurchmessern an der Grenze zwischen beiden Porensystemen korreliert, besser wiedergegeben.

\subsection{Vergleich zwischen den Parametern der Retentionskurve und den bildanalytisch gemessenen fraktalen Dimensionen}

Der von Brooks \& Corey (1964) als Porengrößenindex beschriebene Parameter $\lambda$ kann nach Perrier et al. (1996) basierend auf den Arbeiten von Ahl \& Niemeyer (1989) und Rieu \& Sposito (1991 a,b,c) in Bezug auf eine fraktale Porengrößenverteilung beschrieben werden. Wird der residuale Wassergehalt $\theta_{\mathrm{r}}$ vernachlässigt, erhält G1. 2.28 die folgende Form: 


$$
\theta(\psi)=\theta_{\mathrm{S}}\left(\frac{\psi_{\mathrm{b}}}{\psi}\right)^{\lambda}
$$

Der Exponent kann dann durch $\lambda=\mathrm{E}-\mathrm{D}$ ausgedrückt werden, wobei $\mathrm{E}$ die einbettende euklidische Dimension und D die fraktale Dimension ist. Wird $E=3$ gesetzt und D ebenfalls auf den dreidimensionalen Raum bezogen, ergeben sich gute Übereinstimmungen von gemessenen und berechneten Retentionskurven (Perrier et al. 1996).

Für das in Tab. 6.18 angegebene $\lambda$ ergibt sich mit $D=3-\lambda$ eine fraktale Dimension von $\mathrm{D} \approx 2,876$. Werden die Beziehungen zwischen der Parametrisierung nach Brooks und Corey und der von van Genuchten berücksichtigt (s. Kapitel 2.2.1), so ergibt sich aus

$$
\lambda=\mathrm{n}-1=3-\mathrm{D}
$$

für n die einfache Gleichung

$$
\mathrm{n}=4-\mathrm{D} \text {. }
$$

Damit lassen sich die fraktalen Dimensionen des Parameters $\mathrm{n}$ der Retentionskurve nach van Genuchten berechnen, und es ergeben sich folgende Werte:

Tab. 6.19: Aus den Parametrisierungen der Retentionskurve berechnete fraktale Dimensionen.

\begin{tabular}{|c|cc|}
\hline Brooks und Corey & $\lambda=0,12412$ & $\mathrm{D} \approx 2,876$ \\
einfache van Genuchten Gleichung & $\mathrm{n}=1,16173$ & $\mathrm{D} \approx 2,838$ \\
van Genuchten für $\mathrm{pF}<2$ & $\mathrm{n}=1,07260$ & $\mathrm{D} \approx 2,927$ \\
van Genuchten für $\mathrm{pF} \geq 2$ & $\mathrm{n}=1,25352$ & $\mathrm{D} \approx 2,746$ \\
\hline
\end{tabular}

Diese stimmen gut mit den bildanalytisch gemessenen D überein, besonders wenn der jeweilige Porengrößenbereich berücksichtigt wird. Wird die aus dem Porengrößenverteilungsindex $\lambda$ (nach Brooks und Corey) berechnete fraktale Dimension mit den durch das robuste Schätzverfahren ermittelten $\mathrm{D}_{2}$ verglichen (Tab. 6.4, 6.5 und 6.6), ergibt sich eine gute Übereinstimmung. Dies gilt vor allem für die mit dem Boxcounting-Verfahren ermittelten Werte, die von $D_{2}^{b}=1,862$ bis $D_{2}^{b}=1,897$ reichen. Die aus dem Parameter $\mathrm{n}$ der einfachen van Genuchten-Gleichung berechnete fraktale Dimension stimmt eher mit den Werten des Schliffs Leo9 für Dilatation überein: $\mathrm{D}_{2}^{\mathrm{d}}=1,841$ für lineare bzw. $\mathrm{D}_{2}^{\mathrm{d}}=1,829$ für exponentielle Schrittweiten. Bei der Anpassung einer doppelten van Genuchten-Gleichung für Poren mit Durchmessern größer als $30 \mu \mathrm{m}$ ist $\mathrm{D}=2,927$, was in der Größenordnung von $\mathrm{D}_{\mathrm{Smax}}$ der empirischen Funktion (D als Funktion der Bildauflösung, s. Tab. 6.14) liegt. Im Bereich der kleineren Poren, die den unteren Ast der Retentionskurve bestimmen, trifft das abgeleitete $\mathrm{D}=2,746$ exakt den mit Boxcounting und exponentiellen Schrittweiten gemessenen Wert über alle Vergrößerungsstufen von Leo8 (s. Tab. 6.5). 
Auch die Übereinstimmung mit der in Tab. 6.16 gezeigten fraktalen Dimension von $\mathrm{D}^{\mathrm{P}}=0,854$ für die distributive Porengrößenverteilung (Mittelwerte an 10 vertikalen Dünnschliffen, s. Abb. 6.24) ist gut, wenn die Differenz um den Summanden „2" nicht berücksichtigt wird.

\subsection{Zusammenfassung der Ergebnisse}

Die Vielzahl der in Kapitel 6 vorgestellten Ergebnisse soll hier zusammengefasst werden, um die wichtigsten Erkenntnisse hervorzuheben. Mit dem FE-REM wurden an drei Dünnschliffen je ca. 100 Bilder verschiedener Vergrößerungsstufen aufgenommen. Die Schliffe Leo8 und Leo9 waren horizontal, Leo7 vertikal ausgerichtet. Die Aufnahmen von Leo9 wurden mit einer höheren Abtastrate gerastert. Leo7 und Leo8 stammten aus einem Kubiena Kasten. Für 15 weitere Dünnschliffe dieses Kastens wurden mit einem Polarisationsmikroskop Aufnahmen erstellt und untersucht. Die Übersichtsbilder der ebenfalls für FE-REM Aufnahmen verwendeten Schliffe tragen die Namen Wild7, Wild8 und Wild9.

\subsubsection{Die fraktale Dimension der Porenraumgrenzlinie}

Das Hauptergebnis der Messung der fraktalen Dimension (D) der Porenraumgrenzlinie mit den Methoden Boxcounting und Dilatation ist der extrem hohe Wert für D. Mit Werten von bis $\mathrm{zu} \mathrm{D}_{\mathrm{L}}=1,935$ ist fast die euklidische Dimension von 2 erreicht. Das heißt, die Grenzlinie zwischen Poren und Matrix im untersuchten Boden ist so komplex, dass sie nahezu die nächsthöhere Dimension erreicht.

Ein weiteres wichtiges Ergebnis ist, dass D mit steigender Vergrößerung bzw. Auflösung abnimmt. Diese Abnahme äußert sich in einer systematischen Abweichung von einer Geraden im log-log-Plot und ist für hohe Vergrößerungsstufen besonders ausgeprägt. Der Theorie entsprechend hätte ein fraktales, also über mehrere Größenordnungen selbstähnliches Objekt, mit höherer Auflösung feinere Strukturen erkennen lassen und D müsste demnach steigen. Die asymptotische Abweichung im Skalierungsbereich weniger Pixel wurde auch von anderen Autoren (Banerji 1987, Rigaut et al. 1983) beobachtet und u. A. als Messfehler interpretiert. Orford und Whalley schlugen (1983) unten aufgeführte Erklärung vor. Sie vermaßen die Umrisse einzelner Partikel mit unterschiedlich großen Schrittweiten (Yardstick- oder Zirkelmethode) und fanden den in Abb. 6.32 dargestellten Zusammenhang: 


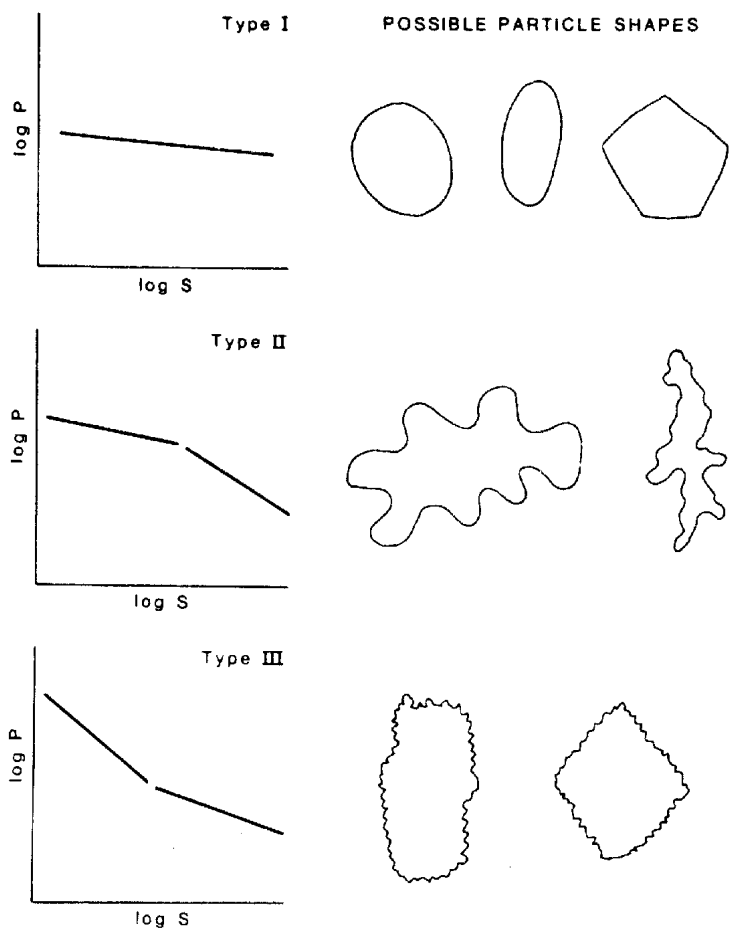

Abb. 6.32: Schematische Darstellung von gemessenen fraktalen Dimensionen für drei typische Partikelformen. Dargestellt ist die Länge des Umrisses über der Schrittweite für dessen Messung. Typ I zeigt Partikel von regelmäßiger Form, die zu einer gleichmäßigen, geringen Steigung im log-log-Plot führen. Partikel vom Typ II haben eine dominante äußere unregelmäßige Struktur bei ebenmäßiger Form des Umfangs (Textur) und führen zu einem konvexen Verlauf im log-log-Plot. Partikel vom Typ III sind geprägt durch eine ebenmäßige Struktur und eine unregelmäßige Umrisslinie und führen zu einem konkaven Verlauf des log-log-Plots. Aus Orford \& Whalley 1983.

Die im Rahmen dieser Arbeit durchgeführten Messungen zeigen für die log-log-Plots der aus allen Vergrößerungsstufen kombinierten FE-REM Aufnahmen (s. Abb. 5.7, 6.5, A.3 und A.4) eine Form vom Typ 2. Der konkave Verlauf wird dem entsprechend als Ausdruck zweier verschiedener Fraktalitäten interpretiert: Der texturellen Fraktalität im Bereich kleiner Skalierungselemente, wo die Oberflächenrauhigkeit einzelner Partikel gemessen wird und der strukturellen Fraktalität im Bereich großer Skalierungselemente, wo die äußere Form der Partikel und insbesondere ihre Anordnung zueinander erfasst wird.

Um einen Messfehler auszuschließen, wurde großen Wert auf die sorgfältige Wahl des für die Berechnung der Steigung relevanten Messbereichs (Cutoffs) gelegt. Die Datensätze der verschiedenen Vergrößerungsstufen wurden so angepasst, dass sich ein kontinuierlicher Datensatz ergab. Daneben wurden auch exponentielle Schrittweiten verwendet, da lineare Schrittweiten in logarithmischer Auftragung zu einer Häufung der Daten im Bereich großer Skalierungselemente führen, der dann stärker zur Steigung beiträgt. Für die so erhaltenen Datensätze wurde eine lineare Regression mit einem robusten Schätzverfahren (lokale M-Schätzer) durchgeführt, das die absoluten Abweichungen optimiert und bessere Anpassung für langschwänzige Verteilungen liefert als die Methode der kleinsten Abweichungsquadrate.

Die systematische Abweichung der Messdaten von einer Gerade blieb bestehen. So kann davon ausgegangen werden, dass die fraktale Dimension der Porenraumgrenzlinie im REM-Bereich $(0,5$ bis $200 \mu \mathrm{m})$ tatsächlich unterschiedliche Muster erfasst. Diese werden 
der Terminologie von Orford \& Whalley entsprechend in textuerelle (die Oberfläche der Partikel betreffend) und strukturelle (die Gesamtform der Partikel betreffend) bezeichnet. Vor allem drückt die hohe Komplexität der Grenzlinie im Bereich großer Skalierungselemente die Anordnung der Partikel zueinander und die Form des Netzwerks aus.

Um den Größenbereich zu bestimmen, der die Grenze zwischen textureller und struktureller Fraktalität bildet, wurden mit dem robusten Schätzverfahren zwei Geraden an die kombinierten Datensätze angepasst und ihre Schnittpunkte berechnet. Diese liegen zwischen 5 und $17 \mu \mathrm{m}$ (Größe des skalierenden Elementes) und können den Porendurchmessern zugeordnet werden, die sich aus der Anordnung der Körner der Hauptkörnungsfraktion (Grobschluff, Durchmesser $20-63 \mu \mathrm{m}$ ) ableiten lassen. Laut Kuntze et al. (1984), zitiert in Friedrichs (1999) besteht ein geometrischer Zusammenhang zwischen Teilchendurchmesser und Kapillarendurchmesser: Wird die Form der Bodenteilchen als Kugel approximiert, gibt es hinsichtlich ihrer Anordnung zwei Extrema. Eine lockere, würfelförmige und eine dichte, hexagonale Anordnung. Für die Anordnungen dieser Kugelpackungen können folgende Beziehungen zwischen Teilchenradius $\mathrm{r}_{\mathrm{T}}$ und Kapillarenradius $\mathrm{r}_{\mathrm{C}}$ errechnet werden:

würfelförmig: $\mathrm{r}_{\mathrm{C}}=\mathrm{r}_{\mathrm{T}} \cdot(\sqrt{2}-1)$

hexagonal: $\quad \mathrm{r}_{\mathrm{C}}=\mathrm{r}_{\mathrm{T}} \cdot\left(\frac{2 \cdot \sqrt{3}}{3}-1\right)$

Für den Grobschluff ergeben sich demnach Kapillarendurchmesser von 3,09 bis 8,28 $\mu \mathrm{m}$ für die kleinsten und 9,75 bis 26,10 $\mu \mathrm{m}$ für die größten Partikel. Geht man von einer Lagerung aus, die zwischen den oben aufgeführten Extrema liegt, ergeben sich Kapillarendurchmesser von 5,69 bis $17,93 \mu \mathrm{m}$. Dies korreliert sehr gut mit den oben angeführten Schnittpunkten zwischen textureller und struktureller Fraktalität.

Über die Anpassung von zwei Geraden hinaus wurde eine kontinuierliche Funktion angepasst, die die Abhängigkeit der gemessenen fraktalen Dimension von der Bildauflösung bzw. dem mittleren logarithmischen Cutoff jeder Vergrößerungsstufe beschreibt. Für die drei untersuchten Dünnschliffe und die Methoden Boxcounting und Dilatation ergibt sich für die fraktale Dimension der Oberfläche $D_{S}\left(D_{S}=D_{L}+1\right)$ eine minimale und eine maximale fraktale Dimension: $\mathrm{D}_{\mathrm{Smin}}=2,558$ und $\mathrm{D}_{\mathrm{Smax}}=2,984$.

Die Auswertung der lichtmikroskopisch aufgenommen Bilder ergibt für die horizontal ausgerichteten Schliffe mit $D_{L}=1,779$ eine gering, aber signifikant höhere fraktale Dimension als für die vertikal ausgerichteten Schliffe mit $D_{L}=1,771$ (jeweils für das Boxcounting-Verfahren). Interessanterweise stimmen diese Ergebnisse, die für den Skalierungsbereich von 600-2500 $\mu \mathrm{m}$ gelten, gut mit der Anpassung einer Geraden für alle REM-Vergrößerungen mit einem Skalierungsbereich von 0,05-200 $\mu \mathrm{m}$ überein. Unterhalb einer Größe des skalierenden Elementes von ca. $600 \mu \mathrm{m}$ zeigen die lichtmikroskopischen Bilder keine Fraktalität, da die Ränder der abgebildeten Poren glatt erscheinen. Oberhalb von $600 \mu \mathrm{m}$ wird die Anordnung der Poren zueinander erfasst. 


\subsubsection{Die Messung der Porengrößenverteilung}

Die Porengrößenverteilung wurde an den FE-REM Bildern der Dünnschliffe Leo7 und Leo8 sowie an den lichtmikroskopischen Bildern von Schliffen unterschiedlicher Ausrichtungen eines Kubiena Kastens gemessen. Der Vergleich von kumulierter Porenverteilung mit der für denselben Boden erstellten Retentionskurve zeigt für kleine Poren eine deutliche Überschätzung der Volumenanteile durch die Retentionskurve (s. Abb. 6.18 und 6.19). Dies kann mit dem Flaschenhalseffekt erklärt werden, der besagt, dass bei verbundenen Poren immer die kleinste ausschlaggebend für den zur Entwässerung benötigten Unterdruck ist. Die am vertikal ausgerichteten Schliff Leo7 bildanalytisch gemessene Verteilung schneidet die Retentionskurve bei ca. $270 \mathrm{hPa}$ bzw. 11,11 $\mu \mathrm{m}$ Porendurchmesser. Die am horizontal ausgerichteten Schliff Leo 8 gemessene Porenverteilung verläuft zwischen 70 und $100 \mathrm{hPa}$ parallel zur Retentionskurve. Der stark abfallende Ast verläuft für Leo7 steiler als für Leo8. Für große Poren liegt die kumulierte Porenverteilung über der Retentionskurve. Damit kann gezeigt werden, dass aus der Retentionskurve nicht auf die Porenverteilung geschlossen werden kann, weil die Konnektivität des Porensystems bei der Desorption mit erfasst wird. Dies deckt sich mit den Erkenntnissen von Vogel (2000), der mit einem numerischen Experiment zeigt, dass für völlig unterschiedliche Porengrößenverteilungen identische Retentionskurven erzeugt werden können.

Zusammen mit den lichtmikroskopischen Aufnahmen der entsprechenden Dünnschliffe ergibt sich eine Gesamtporenfläche von 47 bzw. $46 \%$, der höchste gemessene Wassergehalt beträgt 44,10 Gew. \%. Aus Lagerungsdichte und Dichte der Festsubstanz wurde ein Gesamtporenvolumen von 40,90 Vol. \% berechnet, was für einen Lößboden recht gering erscheint. Wenn berücksichtigt wird, dass diese Angaben mit völlig unterschiedlichen Methoden ermittelt wurden, stimmen die Angaben recht gut überein.

Die distributive Porengrößenverteilung zeigt ein Maximum für die kleinsten Poren bei einem Durchmesser von rund 1,50 $\mu \mathrm{m}$. Die Form der Verteilung deutet auf eine (log-log) Normalverteilung hin (Abb. 6.20 und 6.21), wobei Porendurchmesser $<1,50 \mu \mathrm{m}$ nicht oder nicht hinreichend genau erfasst werden konnten. Die lichtmikroskopischen Bilder zeigen ein zweites Maximum für Poren mit Durchmessern von 200 bis $600 \mu \mathrm{m}$. Dies deutet auf ein Sekundärporensystem hin, das u. A. für die hohen Wassergehalte bei Sättigung verantwortlich ist, die mit der Parametrisierung der Retentionskurve nicht erfasst werden konnten. Solch ein Struktur-Porensystem findet sich laut Durner (1991, Kapitel 4) in unbearbeiteten Böden jeder Textur, was bei dem untersuchten Waldboden gegeben ist.

\subsubsection{Vergleich mit der Parametrisierung der Retentionskurve}

$\mathrm{Zu}$ Beginn entwässert der Boden recht langsam und zeigt keinen deutlichen Lufteintrittspunkt. Ab ca. $30 \mathrm{hPa}$ nimmt die Entwässerung deutlich zu, bei $14000 \mathrm{hPa}$ beträgt der Wassergehalt immer noch über 18 Vol. \%. Der flache Kurvenverlauf nahe der Sättigung und das langsame Abflachen in Richtung großer Wasserspannung wird durch den hohen Tonanteil ( $>20$ Gew. \%) wesentlich beeinflusst. Bei Wassersättigung quillt der Ton und verkleinert so die Porendurchmesser, während bei hohen Saugspannungen immer noch Wasser in den Feinporen vorhanden ist. Der dazwischen liegende Bereich der stärksten Entwässerung wird durch ein Netzwerk aus körnungsbedingten Poren der 
Grobschlufffraktion geprägt, das für Wasserspannungen von rund 167 bis $527 \mathrm{hPa}$ besteht (s. Gl. 6.10 und 6.11). Genau in dem Bereich der entsprechenden Porendurchmesser liegt auch die Grenze zwischen struktureller und textureller Fraktalität.

Die Parametrisierung der Retentionskurve zeigt für den Ansatz nach van Genuchten (s. Abb. 6.29) eine wesentlich bessere Anpassung als nach Brooks und Corey (s. Abb. 30). Um die im Labor gemessenen ungesättigten Wasserleitfähigkeiten mit der von Mualem vorgeschlagenen Funktion (Gl. 2.27) wiedergeben zu können, erbringt ein bimodaler Ansatz für die Retentionskurve die bessere Übereinstimmung. Die dafür berechneten van Genuchten Parameter weisen für den sättigungsnahen Bereich auf eine gröbere Textur hin als die für den sättigungsfernen Bereich $(\geq 100 \mathrm{hPa})$ Bereich.

Der Parameter $n$ der van Genuchten-Gleichung steht ähnlich wie der Exponent von Brooks und Corey für die Porengrößenverteilung. Je größer n gewählt wird, desto steiler verläuft der abknickende Ast der Retentionskurve. Über die Beziehung 4-n=D kann dem Anpassungsparameter eine fraktale Dimension zugeordnet werden. Der einfache van Genuchten Ansatz ergibt $D=2,838$ und der doppelte für den sättigungsnahen Bereich $\mathrm{D}=2,927$ und für den sättigungsfernen $\mathrm{D}=2,746$. Besonders die Ergebnisse aus dem bimodalen Ansatz stimmen mit den bildanalytisch gemessenen Dimensionen der Porenraumgrenzlinie gut überein. Die höhere fraktale Dimension entspricht der hohen Komplexität der Grenzlinie zwischen Pore und Matrix für Poren mit Durchmessern von bis zu $200 \mu \mathrm{m}$. Die niedrigere (wenngleich mit $\mathrm{D}=2,746$ immer noch etwas höher als die des Menger-Schwamms von 2,727) entspricht etwa der Dimension, die über alle Vergrößerungsstufen der REM-Aufnahmen gemessen wurde. Somit kann gezeigt werden, dass sich die bildanalytisch gemessenen D direkt mit aus der Desorptionskurve abgeleiteten fraktalen Dimensionen vergleichen lassen, wobei insbesondere der Trend zu höherer Komplexität des Porensystems für gröbere Bereiche im untersuchten Boden bestätigt werden kann. 



\section{$7 \quad$ Diskussion}

Die Diskussion ist gegliedert in einen methodischen Teil und die Interpretation der Ergebnisse. Die Diskussion der präparativen Methoden erfolgt in der Reihenfolge der durchgeführten Arbeitsschritte bis zur Erstellung des digitalen Bildes, an dem die bildanalytische Weiterverarbeitung (Segmentierung) und Messung erfolgte. Die klassischen bodenphysikalischen Methoden werden einschließlich der Parametrisierung der Retentionskurve besprochen.

\subsection{Diskussion der verwendeten präparativen und mikroskopischen Methoden}

Die sehr lange Aushärtezeit des Polyesterharzes von mehreren Monaten verzögerte die Weiterverarbeitung der Proben erheblich. Versuche mit einer Verminderung des Styrolanteils und höheren Zugaben von Härter und Beschleuniger gingen aber zu Lasten der Präparationsqualität. Ein weiteres methodisches Problem stellt die Lufttrocknung der Proben dar: Da die tonhaltige Matrix schrumpft, verändert sich bereits das Porensystem. Daher wurden wenige Proben feldfrisch in das Aceton überführt, allerdings verringerte sich die Präparationsqualität erheblich, die Proben waren nicht vollständig mit Vestopal durchtränkt. Bei der Verwendung dieses Harzes wird also bei einer langen Aushärtzeit eine sehr gute Durchtränkung der Probe erreicht, während die durch die Lufttrocknung erfolgte Veränderung des Porenraums in Kauf genommen werden sollte. Für die Erstellung von Dünnschliffen können kleinere Bodenvolumina eingegossen werden, was die Aushärtzeit wesentlich verkürzen sollte.

Ein von Protz \& VandenBygaart (1998) vorgeschlagenes Verfahren, von einem Bildausschnitt des Dünnschliffs je eine Aufnahme im Durchlicht, mit gekreuzten Polarisatoren und im Auflicht zu erstellen, lässt sich mit gescannten Dias im Sinne einer pixelgetreuen Abbildung nicht durchführen. Die Autoren arbeiten mit einer digitalen Kamera und weisen ausdrücklich darauf hin, dass der Schliff nicht bewegt und nur die Lichtquelle bzw. der Strahlengang verändert wird. So liegen ihre Abbildungen exakt übereinander und die Bilder können morphometrisch verschnitten werden. Bei eingescannten Dias ist es auch bei identischen Bildausschnitten nicht möglich, die Dias so exakt im Scanner zu positionieren, dass die erhaltenen Bilder Pixel für Pixel den gleichen Bildausschnitt wiedergeben.

Während im Lichtmikroskop (LM) mit demselben Objektiv und Okular immer dieselbe Vergrößerungsstufe erzielt wird ist dies beim FE-REM nicht der Fall. Es kann zwar eine Vergrößerungsstufe eingestellt werden, die tatsächliche Vergrößerung wird aber durch die Anregungsspannung und den Arbeitsabstand mit beeinflusst. Beim verwendeten Leo Gemini 1530 bezieht sich die angegebene Vergrößerungsstufe auf den als Standard eingerichteten Drucker, was zu Abweichungen von Vergrößerungsstufe und Auflösung führen kann. Das nichtlineare Verhältnis beider Größen, wie es sich in Tab. 4.1, Spalte Leo Gemini, Bildgröße 1024 x 768 Pixel zeigt, basiert auf der Tatsache, dass die Bilder mit den Vergrößerungsstufen 1200x, 1500x und 5000x zu einem späteren Zeitpunkt angefertigt wurden und ein anderer Drucker als Standard eingerichtet war. Die tatsächliche Vergrößerung am REM kann nur über die Messung des Kalibrierbalkens ermittelt werden. 
Mit einem Eichpräparat (ein konstantes Kohlenstoffgitter) wurde der in jeder REM Aufnahme eingeblendete Kalibrierbalken überprüft. Es konnte feststellt werden, dass dieser in jedem Fall die korrekte Vergrößerung wiedergibt.

Die für das FE-REM verwendete Beschleunigungsspannung von $18 \mathrm{kV}$ ist für das verwendete Gerät immer noch recht hoch. Es können niedrigere Spannungen von bis zu 1 $\mathrm{kV}$ erreicht werden, was sich bei den durchgeführten Untersuchungen jedoch nicht als notwendig erwies. Um bei hohen REM Vergrößerungen das Durchscheinen tiefer liegender Matrixanteile durch das Gießharz zu vermeiden, könnte mit einem größeren Anteil an Sekundärelektronen, deren Eindringtiefe geringer ist als die der Rückstreuelektronen, gearbeitet werden.

Um sehr kleine Skalierungsbereiche zu erfassen, muss die Abtastrate des FE-REM erhöht werden, eine höhere Vergrößerung bringt weniger Informationsgewinn. So wird für Leo7 mit der höchsten Vergrößerungsstufe kein weiterer Bereich als mit der zweithöchsten erreicht, bei Leo8 zusätzliche 0,50 $\mu \mathrm{m}$. Erst die mit höherer Abtastrate aufgenommenen Bilder der Serie Leo9 bringen einen deutlich niedrigeren unteren Cutoff für die höchste Vergrößerungsstufe (s. Tab. 6.1, 6.2 und 6.3).

\subsection{Diskussion der digitalen Bildanalyse}

Mit dem verwendete Programmsystem KS400 und den dafür entwickelten Programmen konnte nach erfolgter Binarisierung mit einer enormen Genauigkeit gemessen werden. Beim Boxcounting-Verfahren handelt es sich um eine etablierte Methode, die bereits häufig zur Messung der fraktalen Dimension der Bodenstruktur eingesetzt wurde (s. Bartoli et al. 1991, 1999, Anderson et al. 1996, Pachepsky et al. 1996, Giménez et al. 1997, Ogawa et al. 1999). Das Dilatations-Verfahren wird nicht so häufig angewendet, hier war Flook (1978) der Erste, der das Verfahren in der digitalen Bildverarbeitung verwendete. Diese Methode wurde später von Eins (1995) verbessert und von Dathe et al. (2001) zur Charakterisierung der Bodenstruktur benutzt. Es ergab sich, dass mit dem BoxcountingVerfahren exakter im Bereich weniger Pixel und mit dem Dilatations-Verfahren im Bereich großer Strukturelemente gemessen werden kann. Die Dilatation benötigt eine höhere Rechenzeit, bei der für die Serie Leo9 verwendeten hohen Auflösung in Kombination mit sehr komplexen Bildern wird häufig die Kapazität des internen Arbeitsspeichers des verwendeten Systems erreicht und das Programm bricht ab.

Es ist nicht trivial, aus den erhobenen Rohdaten (skalenabhängige Anzahl der Kästchen für Boxcounting bzw. Länge der Umhüllenden für Dilatation) die Steigung des RichardsonPlots $\mathrm{zu}$ bestimmen. Natürliche Strukturen sind nicht über mehrere Größenordnungen selbstähnlich. Häufig kann daher eine gleichmäßige Steigung nur in einem begrenzten Skalierungsbereich beobachtet werden oder es sind mehrere lineare Abschnitte vorhanden. Besonders groß ist die Abweichung nahe der Auflösungsgrenze und es muss entschieden werden, welche Daten zur Berechnung beitragen sollen (Bestimmung der Cutoffs).

Bei der Messung der Porengrößenverteilung werden die größeren Poren zunächst vom Rand her dilatiert bis die mittlere Pore annährend der Form des Strukturelementes entspricht. So werden raue Randbereiche einer relativ großen Pore kleineren Porengrößenklassen zugeordnet, obwohl sie eigentlich zu der großen Pore gehören. Dies 
führt zu einer Überschätzung der Porengrößenverteilung für kleine Poren der jeweiligen Auflösungsstufe. In Hinblick auf den Wassertransport im Boden könnte diese durch die Messung hervorgerufene Ungenauigkeit allerdings gerechtfertigt werden: Bei hohen Saugspannungen, also kleinen Porenradien, kann sich das Bodenwasser auch an den Wänden großer Poren befinden, besonders wenn diese unregelmäßig geformt und nicht vollständig mit Wasser gefüllt sind. Die Zählung der einzelnen Poren diente vor allem der Kontrolle des Messalgorithmus. Die erhaltene Anzahl stellt bei den REM-Vergrößerungen keinen physikalisch relevanten Wert dar, da der Porenraum so hochgradig vernetzt erscheint, dass einzelne Poren nur im Bereich einzelner weniger Pixel oder für geringe Vergrößerungsstufen (s. Abb. $4.7 \mathrm{a}$ und b) erfasst wurden.

Die bildanalytische Messung der Porengrößenverteilung stellt ein Verfahren dar, das in der Bodenkunde nur selten angewendet wird (Vogel 1996). Um eine physikalisch nachvollziehbare Grundlage zur Modellierung der Retentionskurve zu schaffen wird jedoch genau diese Methodik von Perrier et al. (1996) vorgeschlagen, wenn die Einschränkungen, die mit einer Messung im zweidimensionalen Raum verbunden sind, überwunden werden können.

\subsection{Diskussion der verwendeten mathematischen und statistischen Methoden}

Die Bestimmung des linearen Bereichs im Richardson-Plot durch die Berechnung eines optimalen Korrelationkoeffizienten (unterer Cutoff für Boxcounting) hat sich als eine robuste Methode erwiesen. Für Dilatation kann altenativ zur Berechnung des Differenzenquotienten ein definierter Korrelationskoeffizient als Entscheidungskriterium für die Wahl des verwendeten Datenbereichs vorgegeben werden. Sowohl für die REMals auch für die LM-Aufnahmen konnte festgestellt werden, dass die Differenzen für D sowohl zwischen den verwendeten Methoden als auch zwischen den beiden untersuchten Ausrichtungen der Dünnschliffe größer werden, wenn der Richardson-Plot auf Daten eines einheitlichen Datenbereichs (innerhalb der Cutoffs) für unterschiedliche Messserien bezogen wird.

Für die Messung an aus beiden mikroskopischen Verfahren gewonnenen Bildern ist fast immer die mit Boxcounting gemessene Dimension kleiner als die mit Dilatation gemessene. Ein möglicher Grund ist darin zu sehen, dass sich die Angabe der Größe des strukturierenden Elements auf die Seitenlänge der Box bzw. auf den Durchmesser des für Dilatation verwendeten Kreises bezieht. Um den größten, im Quadrat möglichen Durchmesser $\mathrm{zu}$ erhalten muss jedoch die Seitenlänge mit $\sqrt{2}$ multipliziert werden. Demnach wäre die Größengabe des Strukturelements für Boxcounting immer etwas zu niedrig, wobei der tatsächliche Wert zwischen der Diagonalen und der Seitenlänge liegt.

Die Mittelwertbildung der Steigungen im Richardson-Plot für alle Bilder einer Vergrößerungsstufe und eines Schliffs (Methode A) ist für die Bestimmung der Varianz zwischen den Einzelbildern unerlässlich. Soll nicht auf die Besonderheiten einzelner Bilder eingegangen werden, bietet sich für die Bestimmung der fraktalen Dimension die Methode B (Mittelwertbildung der Rohdaten, ein Richardson-Plot pro Vergrößerungsstufe), kombiniert mit der Optimierung des Korrelationskoeffizienten für den unteren Cutoff, an. 
Dann ist es sinnvoller, als oberen Cutoff für alle Bilder einer Vergrößerungsstufe eine einheitliche Größe des Strukturelements zu wählen. So wird vermieden, dass einzelne Messreihen nicht in die Mittelwertbildung einbezogen werden können, wenn ihre fraktale Dimension schon nach relativ wenigen Messschritten hinreichend genau gemessen wurde.

Zur Berechnung der asymptotisch verlaufenden Funktion, die die Abhängigkeit der gemessenen fraktalen Dimension von der Bildauflösung beschreibt, sei Folgendes gesagt: Bei der Anpassung der Parameter der empirischen Gleichung an die Messwerte kann die Optimierung der Schätzung (durch Minimierung der Abweichungsquadrate in y-Richtung) zwangsläufig zu Abweichungen von den gemessenen D der höchsten Auflösungsstufe nahe der y-Achse führen. So ist für die Abb. $6.15 \mathrm{c}$ auch eine Kurve denkbar, die bei einem wesentlich geringeren $\mathrm{D}$ für Auflösungen nahe 0 beginnt oder sich an die $\mathrm{y}$-Achse asymptotisch anschmiegt. Dann wären die Abweichungen $\mathrm{zu}$ den gemessenen $\mathrm{D}$ in $\mathrm{x}$ Richtung gering, was nur mit einem anderen Optimierungsverfahren möglich ist.

Bei der Summierung der Porengrößenverteilungen verschiedener Vergrößerungsstufen eines Schliffs muss beachtet werden, dass sich die Wahl des Verknüpfungspunktes stark auf den Verlauf der Gesamtkurve auswirkt. Der Grund ist, dass die Kurven nicht einfach parallel verschoben verlaufen, sondern häufig in Richtung größerer Porendurchmesser konvergieren. Daher wurde großen Wert auf eine nachvollziehbare Verknüpfung der Datenreihen gelegt. Die Addition erfolgte an exakt dem Punkt, wo der Variationskoeffizient der jeweils höheren Vergrößerungsstufe $<100 \%$ wurde. Eine andere Möglichkeit, die kumulative Verteilung zu erhalten, ist die Integration der normierten distributiven Verteilungen (s. Abb. 6.20 und 6.21).

\subsection{Diskussion der bodenphysikalischen Methoden}

Wie für physikalische Messmethoden allgemein gilt auch hier, dass die Wahl der verwendeten Methode das Ergebnis beeinflussen kann. So ist die gesättigte Leitfähigkeit auch eine inverse Funktion der Länge der durchflossenen Bodensäule. Je höher die verwendeten Stechzylinder, desto geringer wird die gesättigte Leitfähigkeit (Anderson \& Bouma 1973). Der Grund ist in der mit zunehmender Fließstrecke wachsenden Wahrscheinlichkeit der Isolierung des Grobporensystems zu sehen. Bei der Erstellung der Retentionskurve kann sich der Wechsel von der Unterdruck- auf die Überdruckmethode, der in der vorliegenden Arbeit bei $316 \mathrm{hPa}$ stattfand, in den Ergebnissen widerspiegeln. Maximal kann bis zur Höhe des Luftdrucks (rund $1000 \mathrm{hPa}$ ) mit Unterdruck gearbeitet werden, in der Spanne der angegebenen Bereiche liegt jedoch die größte Steigung der Retentionskurve. So können Abweichungen von einem gleichförmigen Verlauf der Retentionskurve, wie sie bei strukturierten Böden beobachtet werden, fälschlicherweise auf einen Messfehler zurückgeführt werden (Durner 1991, Kap. 4).

Wichtig ist immer die Vorgeschichte bezüglich Be- und Entwässerung eines auf hydrologische Eigenschaften untersuchten Bodens (s. u. a. Klute 1996). So wird für die Erstellung von Retentionskurven üblicherweise eine Desorption durchgeführt. Bei einer Aufsättigung werden bei gleichen Unterdrücken, bedingt durch den Flaschenhalseffekt und damit verbundene Lufteinschlüsse, geringere Wassergehalte gemessen (Hysterese). Dies kann zu Ungenauigkeiten bei der Berechnung des Wassertransports führen. 
Eine Sättigung der Stechzylinder, wie sie im Labor vor der Erstellung der Desorptionskurve durchgeführt wird, findet in der Natur unter diesen Bedingungen nicht statt. Die $100 \mathrm{~cm}^{3}$ Stechzylinder waren nach der Sättigung einige $\mathrm{mm}$ über den Zylinderrand hoch gequollen, was zu einer Überschätzung des Wassergehalts bei Sättigung führt. Bei den $250 \mathrm{~cm}^{3}$ Stechzylindern, wie sie für die Messung der ungesättigten Wasserleitfähigkeit verwendet wurden, war dies nicht zu beobachten. An diesen wurde ein Sättigungswassergehalt von $41,16 \mathrm{Vol} \%$ gemessen, der eher mit dem berechneten Gesamtporenvolumen von 40,90 Vol. \% korreliert. Da bei den großen Stechzylindern die Gefahr besteht, dass sie nicht vollständig gesättigt waren, wurde für die Parametrisierung der Retentionskurve der höhere Wert der $100 \mathrm{~cm}^{3}$ Proben verwendet.

Die Messung der ungesättigten Wasserleitfähigkeit an der Ku-Apparatur verlief, von einigen Mängeln der Steuerungssoftware abgesehen, relativ schnell und zuverlässig. Das allmähliche Aufsättigen der $250 \mathrm{~cm}^{3}$ Stechzylinder dauerte lange. Gegen Ende des Sättigungsvorgangs wurde ein Vakuum angelegt. Die in den Abb. 6.28 und 6.31 gezeigten Ergebnisse wurden aus den ermittelten Rohdaten gemäß G1. 4.5 berechnet. Die extrem hohe gesättigte Wasserleitfähigkeit, die an den horizontal entnommenen Proben gemessen wurde und die im Gelände beobachtete feine Schichtung des Bodens können einen Grund für die relativ hohen Wassergehalte bei $\mathrm{pF}$ 4,2 darstellen: Ein horizontal verlaufendes Porensystem hemmt den Wassertransport in vertikaler Richtung, wenn die Saugspannung für die Entwässerung der entsprechenden Poren überschritten ist.

Die Parametrisierung der im Labor erhobenen Retentionskurve nach Brooks und Corey erbrachte kein überzeugendes Ergebnis (s. Abb. 6.30). Daher wurden aus diesem Parametersatz keine Wasserleitfähigkeiten berechnet. Die Anpassung nach van Genuchten zeigt eine sehr gute Übereinstimmung mit den gemessenen Werten. Die Unterschiede zwischen der Anpassung einer einfachen und einer doppelten van Genuchten Gleichung zeigen sich vor allem in den aus der Retentionskurve berechneten Leitfähigkeiten.

\subsection{Diskussion der Ergebnisse}

Es ist allgemein bekannt, dass die Bodenstruktur und insbesondere das Porensystem im Boden ein äußerst komplexes System darstellen. Die bildanalytisch gemessene fraktale Dimension der Porenraumgrenzlinie, die als quantitative Maßzahl der Komplexität aufgefasst werden kann, übertraf jedoch die vorher angestellten Überlegungen. So werden Werte von bis zu $D_{L}=1,935$ für die niedrigste Auflösungsstufe der mit dem FE-REM angefertigten Bilder erzielt (s. Tab. 6.3). Dies ist die Dimension einer Linie, gemessen in einer Fläche, weshalb der Wert zwischen 1 und 2 liegen muss. Ein Messwert nahe 2 besagt, dass die Grenzlinie nahezu die gesamte Fläche ausfüllt.

Der Theorie entsprechend war mit einem wachsenden D für höhere Auflösungen gerechnet worden, weil dann auch feinste Strukturen erkannt werden können. Allerdings weisen natürliche fraktale Objekte immer Grenzen der Selbstähnlichkeit auf. Die im Rahmen dieser Arbeit durchgeführten Messungen ergeben alle ein kleineres D für höhere Auflösungen. Als Erklärung wird postuliert, dass bei der Messung der fraktalen Dimension der Grenzlinie zwischen Poren und Matrix sowohl die Rauhigkeit der Kurve als auch die Anzahl der Verzweigungspunkte erfasst wird. Dieser Sachverhalt deckt sich mit den Erkenntnissen anderer Autoren. Giménez et al. (1997) beschreiben, dass $\mathrm{D}_{\mathrm{S}}$ für kleine 
Boxgrößen Informationen über die Porenrauhigkeit enthält und für große Boxgrößen sensitiv für die räumliche Anordnung der Poren ist. Anderson et al. (1996) zitieren Crawford et al. (1993a) und sprechen von konkurrierenden Effekten von Heterogenität und Tortuosität. Die in der vorliegenden Untersuchung gemessenen fraktalen Dimensionen der Porenraumgrenzlinie korrespondieren mit denen von Anderson et al. (1996), die $\mathrm{D}_{\mathrm{S} 1}=2,15$ bis 2,25 und $\mathrm{D}_{\mathrm{S} 2}=2,91$ bis 2,99 mit der Closing-Methode (Dilatation gefolgt von Erosion) ermittelten, und Bartoli et al. (1999), die $D_{S}=2,61-2,73$ durch Quecksilber-Porosimmetrie berechneten.

Die Anpassung von zwei Geraden über den gesamten mit REM-Aufnahmen abgedeckten Größenbereich impliziert zwei Aspekte (Dathe et al. 2001): (i) Die physikalische Bedeutung des Schnittpunkts der Geraden und (ii) die Frage, ob es sich um zwei oder mehr lineare Abschnitte im Richardson-Plot handelt oder um ein asymptotisches Verhalten. In Sinne von (i) ist die Anpassung von zwei Geraden als erste Approximation zu sehen, die in Kapitel 6.2 beschriebene Funktion für die Abhängigkeit der fraktalen Dimension von der Auflösung stellt einen weiterführenden Ansatz in Sinne von (ii) dar.

(i) Der aus der Anpassung von zwei Geraden berechnete Schnittpunkt wird als Grenze zwischen textureller (im Sinne von Oberflächenrauigkeit einzelner Partikel) und struktureller Fraktalität (Anordnung der Partikel zueinander bzw. Beschreibung des Porennetzwerks) interpretiert. Da die mit lokalen MSchätzern berechneten Anpassungen geringe absolute Abweichung aufweisen, sollte der berechnete Schnittpunkt vorsichtig interpretiert werden. Bei der Betrachtung der REM-Aufnahmen wird deutlich, dass zwei Bereiche des Porensystems existieren, hervorgerufen durch die Anordnung der Schluffkörner und die unregelmäßig verteilte tonige Matrix. Es gibt in der Natur jedoch keinen zwingenden Grund für einen scharfen Übergang zwischen beiden Systemen. Den Messungen zufolge befindet er sich in einer Größenordnung zwischen 5 und $17 \mu \mathrm{m}$.

(ii) Der Richardson-Plot eines ,,asymptotischen Fraktals“, wie er von Rigaut (1984) diskutiert wird, zeigt eine lineare Steigung im Bereich großer Strukturelemente und ein asymptotisches Verhalten für infinitesimal kleine Strukturelemente, das gegen einen konstanten Wert auf der Ordinate tendiert. Was könnte der physikalische Grund für ein derartiges Verhalten sein? Der Schnittpunkt mit der Ordinate steht für die logarithmierte Anzahl der Boxen, die bei einer Seitenlänge von „1“ die Porengrenzfläche beinhalten. Dieser Wert hängt von der Bildgröße und -auflösung $a b$ und wird - im Gegensatz zu den Geradengleichungen - bei der Messung der Porenraumgrenzlinie nicht erreicht. Werden die Daten nicht logarithmiert aufgetragen, wird deutlich, wie sensibel der Skalenbereich für kleine strukturierende Elemente ist.

Zusammenfassend sei gesagt, dass im Bereich weniger Pixel eine auflösungsbedingte Grenze erreicht ist. Strukturen, die unterschiedliche Muster zeigen, hervorgerufen durch verschiedene Prozesse, führen zu verschiedenen fraktalen Dimensionen. Befindet sich ein Muster im Größenbereich der Auflösungsgrenze, überlappen sich beide Phänomene.

Ein Beispiel für die unter (i) diskutierten Sachverhalte kann das für Wild9 verwendete lichtmikrokopische Einzelbild darstellen. Es weist deutlich zwei unterschiedliche 
Strukturbereiche auf (s. Abb. A.2). In dem Gebiet mit der höheren Porosität wird bei der Messung die Anordnung der Poren zueinander schneller erreicht als in dem Gebiet mit der niedrigeren Porosität. So wird der Übergangsbereich zwischen dem Erfassen der Umrisslinie der Poren und ihrer Anordnung zueinander weiter (s. Abb. A.9 b im Vergleich zu A.8 b) und die zweite Gerade erreicht nicht die Steigung, die eine einzelne Gerade im optimalen Skalierungsbereich aufweist.

Der Vergleich zwischen der im Labor gemessenen Retentionskurve mit der bildanalytisch ermittelten kumulierten Porengrößenverteilung weist eindeutig auf die Bedeutung der Konnektivität des Porensystems für den Wassertransport im Boden und das Speichervermögen hin. Für einen Boden mit einer geringeren Spanne der Porengrößenverteilung wäre der Unterschied wohl nicht so gravierend ausgefallen und auch die Anpassung nach Brooks und Corey hätte bessere Ergebnisse geliefert. Die distributive Porengrößenverteilung weist für Poren mit Durchmessern von 1 bis $100 \mu \mathrm{m}$ keine bimodale Verteilung auf, wie sie auf Grund der guten Anpassung der aus den Parametern der doppelten van Genuchten-Gleichung abgeleiteten Wasserleitfähigkeit an die Messwerte angenommen werden könnte. Scheinbar kommt in diesem Teil der Retentionskurve die räumliche Anordnung des durch den Grobschluff geformten Porensystems zum Tragen, ohne dass sich dies in der Porengrößenverteilung widerspiegelt.

Der Vergleich zwischen den aus der Retentionskurve abgeleiteten fraktalen Dimensionen für die einfache und die doppelte van Genuchten-Gleichung und den bildanalytisch gemessenen D zeigt gute Übereinstimmungen. Die für die höchsten Vergrößerungsstufen bildanalytisch gemessenen Werte tauchen allerdings nicht auf, dafür müsste der Anpassungsparameter $\mathrm{n} \approx 1,42$ sein. Dies entspricht ungefähr dem Wert für einen schluffigen Lehm (van Genuchten et al. 1991). Hier zeigt sich eine Schwierigkeit für den Einsatz der bildanalytisch gemessenen D für die Parametrisierung von Retentionskurven: Ein sich verändernder Wert für $\mathrm{n}$ entspricht nicht den Modellvorstellungen. Es könnte sich aber als sinnvoll erweisen, die gemessenen fraktalen Dimensionen der Porenraumgrenzfläche auf ihre Relevanz bezüglich der Tortuosität zu überprüfen. Diese taucht bei der Berechnung der relativen Wasserleitfähigkeit nach Burdine (1953, s. Gl. 2.25) und Mualem (1976, s. Gl. 2.27) als Exponent der relativen Wassersättigung auf. Bei der Anpassung nach Mualem ist der häufig verwendete Wert von $a=0,5$ nur eine mögliche Anpassung, üblicherweise wird $0<\mathrm{a}<2$ angepasst. Roth (1996, Kapitel 5) beschreibt a als zusätzlichen Parameter, der wie die gesättigte Wasserleitfähigkeit $k_{s}$ nicht aus der Retentionskurve abgeleitet werden kann. Vogel (2000) deutet auf die physikalische Relevanz dieses Parameters hin: Positive Werte besagen, dass die Tortuosität mit abnehmender Wassersättigung zunimmt und negative Werte bedeuten das Gegenteil.

Die gemessene Kennzahl D steht jedoch nicht nur für die Tortuosität, sondern beinhaltet auch, wie oben aufgeführt, Aspekte der Heterogenität und Konnektivität. Weitere Untersuchungen sollten in der Richtung erfolgen, diese Größen zu trennen, um z. B. eine konkrete Angabe über Tortuosität und Konnektivität zu erhalten. Die Tortuosität hat die Eigenschaft, dass bei großen fraktalen Dimensionen die Länge der Fließstrecke so sehr zunimmt, dass kein Transport (von z. B. Wasser) mehr stattfindet (Korvin 1992). Die Konnektivität kann bildanalytisch als Anzahl der Verzweigungspunkte und -richtungen gemessen werden. Den Wassertransport im Boden betreffend sollte dies im dreidimensionalen Raum erfolgen. Als theoretische Grundlage bietet sich die Perkolationstheorie an (z. B. Gouyet 1996), mit der die Leitfähigkeit von Netzwerken beschrieben wird. An dem 
Punkt, an dem ein Netzwerk leitend wird, ist es selbstähnlich mit einer charakteristischen Kennzahl, und es steht außer Frage, dass dies im Boden der Fall ist. 


\section{$8 \quad$ Zusammenfassung}

Ein Boden besteht, vereinfacht betrachtet, aus Festsubstanz (Mineralkörnern) und Poren. Die Poren können mit Wasser oder Luft gefüllt sein und bilden ein hochgradig vernetztes System, das für Transportprozesse von großer Bedeutung ist. Die Erfassung des Porensystems erfolgt in der Bodenkunde über die Erstellung der Retentionskurve, die direkt die hydraulischen Eigenschaften der untersuchten Probe wiedergibt. Aussagen über das tatsächlich vorhandene Porensystem können somit bisher nur eingeschränkt getroffen werden. Eine Möglichkeit der direkten Untersuchung bietet die digitale Bildanalyse, die hier an imprägnierten Proben des Tonanreicherungshorizonts einer Parabraunerde aus Löss erfolgt.

Es werden rasterelektronen- und lichtmikroskopische Aufnahmen von Bodendünnschliffen mit unter dem System KS400 (ZeissVision) entwickelten Programmen bildanalytisch untersucht. Der Schwerpunkt liegt auf den rasterelektronenmikroskopischen Aufnahmen. Mit fünf verschiedenen Auflösungsstufen werden Porengrößen von rund 1 bis $200 \mu \mathrm{m}$ erfasst. Der Bereich bis rund $2500 \mu \mathrm{m}$ Durchmesser wird durch die Lichtmikroskopie abgedeckt. Mit dem FE-REM werden an drei Dünnschliffen je ca. 100 Bilder verschiedener Vergrößerungsstufen aufgenommen. Die Schliffe Leo8 und Leo9 waren horizontal, Leo7 vertikal ausgerichtet. Die Aufnahmen von Leo9 wurden mit einer höheren Abtastrate gerastert. Leo7 und Leo8 stammen aus einem Kubiena-Kasten. Für 15 weitere Dünnschliffe dieses Kastens werden mit einem Polarisationsmikroskop Aufnahmen erstellt und untersucht. Zunächst erfolgt die Erstellung eines Binärbildes, wobei jedes Pixel entweder die Information „Pore“ oder die Information „Matrix“ erhält. So kann die Porengrößenverteilung direkt gemessen werden, wobei das Opening-Verfahren zum Einsatz kommt.

Hauptbestandteil dieser Arbeit ist die Messung der fraktalen Dimension der Grenzlinie zwischen Poren und Matrix, im Folgenden Porenraumgrenzlinie genannt. Zum Einsatz kommen das Boxcounting- und das Dilatations-Verfahren, die sich theoretische Aspekte der Selbstähnlichkeit zu Nutze machen, um die Komplexität einer untersuchten Struktur mit einer quantitativen Kennzahl, der fraktalen Dimension (D) zu beschreiben. D drückt das Verhältnis zwischen einem grundlegenden Muster (bzw. einem Generator oder einer Verzweigungsregel) und einer Iterationsregel aus. Viele in der Natur vorkommende Formen können so beschrieben werden, wie z.B. Flusseinzugssysteme, Bäume und Kapillarsysteme von Blutgefäßen. In der Natur stellen fraktale Geometrien die Regel und nicht die Ausnahme dar. Allerdings existieren hier immer eine natürliche untere und obere Grenze der Selbstähnlichkeit, z.B. im Größenbereich von Molekülen.

Es ist ein aufwändiges Verfahren, aus den ermittelten Rohdaten (Anzahl der Boxen für das Boxcounting- bzw. Länge der Ümhüllenden für das Dilatations-Verfahren, jeweils als Funktion der Größe des Strukturelements) die fraktale Dimension zu bestimmen. Zunächst wird für jedes einzelne Bild der Datenbereich ermittelt, der mit einer linearen Regression als log-log-Plot approximiert werden kann. Messwerte für Strukturelemente einer Größe von wenigen Pixeln zeigen eine methodenbedingte Abweichung von einer Geraden. Dieses Phänomen ist in der Literatur bekannt und wird u. a. auf die sogenannte Pixelrauhigkeit zurückgeführt. Für jede Vergrößerungsstufe wird der optimale lineare Bereich innerhalb der Cutoffs bestimmt. 
Ein wesentliches Ergebnis der Messung der fraktalen Dimension (D) der Porenraumgrenzlinie mit den Methoden Boxcounting und Dilatation ist der extrem hohe Wert für D. Mit Werten von bis $z u D_{L}=1,935$ ist fast die euklidische Dimension von 2 erreicht. Das bedeutet, die Grenzlinien zwischen Poren und Matrix im untersuchten Boden sind so komplex, dass sie nahezu die nächsthöhere Dimension erreichen.

Für die einzelnen Vergrößerungsstufen ergeben sich mit höherer Auflösung kleinere Werte für D. Dieses Ergebnis war unerwartet, daher wird das Phänomen eingehend untersucht. Dafür werden die jeweiligen, als optimal angesehenen Rohdaten jeder Vergrößerungsstufe gemittelt und so angepasst, dass sich ein kontinuierlicher Datenbereich ergibt (einzeln dargestellt erscheinen die Geraden der von verschiedenen Vergrößerungsstufen stammenden Bilder parallel verschoben). Zusätzlich wird der Einfluss der Schrittweiten auf die fraktale Dimension untersucht. Eine Möglichkeit ist die Umrechnung der Skalierungsschritte in exponentielle Schrittweiten, was zu gleichen Abständen der Daten im logarithmischen Plot führt.

Die systematische Abweichung der Messdaten von einer Gerade bleibt bestehen. Für hohe Vergrößerungen bzw. Auflösungen nimmt die fraktale Dimension ab, d.h. die Bodenstruktur im Bereich einiger $\mu \mathrm{m}$ ist weniger komplex als im Bereich einiger $100 \mu \mathrm{m}$. Somit ist davon auszugehen werden, dass die fraktale Dimension der Porenraumgrenzlinie im REM-Bereich $(0,5$ bis $200 \mu \mathrm{m})$ tatsächlich unterschiedliche Muster erfasst. Diese werden der Terminologie von Orford \& Whalley (1983) entsprechend in textuerelle (die Oberfläche der Partikel betreffend) und strukturelle (die Gesamtform der Partikel betreffend) bezeichnet. Vor allem drückt die hohe Komplexität der Grenzlinie im Bereich großer Skalierungselemente die Anordnung der Partikel zueinander und die Form des Porennetzwerks aus. An die kontinuierlichen Datensätze werden mit einem robusten Schätzverfahren zwei Geraden angepasst, um den Übergang zwischen beiden Systemen zu quantifizieren. Für die untersuchten Dünnschliffe befindet dieser sich zwischen 5 und 17 $\mu \mathrm{m}$.

Im nächsten Schritt wird für jede Messserie und Methode eine einfache Sättigungsfunktion angepasst. Diese Funktion bietet den Vorteil, dass in Abhängigkeit von der Auflösung eine minimale $\left(\mathrm{D}_{\text {Smin }}\right)$ und eine maximale $\left(\mathrm{D}_{\text {Smax }}\right)$ fraktale Dimension angepasst werden kann. So kann angegeben werden, ab welcher Größe des Strukturelements $D_{\text {Smax }}$ erreicht ist und welchen Wert $\mathrm{D}_{\text {Smin }}$ für infinitisimal kleine Strukturelemente annimmt. Die Relevanz dieser Funktion ist dahingehend zu überprüfen, ob sie sich mit der zweiten Ableitung der Kurve im log-log-Plot beschreiben lässt.

Die Porengrößenverteilung wird als distributive und kumulierte Verteilung dargestellt und die kumulierte Verteilung analog zur Retentionskurve aufgetragen. Für kleine Porendurchmesser ergibt sich eine signifikante Abweichung in Richtung kleiner Poren, deren Volumenanteile von der Retentionskurve deutlich überschätzt werden. Der Grund ist in der Konnektivität der Porensystems zu sehen, die von der Retentionskurve mit erfasst wird. Somit kann gezeigt werden, dass von der Retentionskurve nicht ohne Einschränkungen auf die Porengrößenverteilung geschlossen werden darf. 
Die distributive Porengrößenverteilung zeigt ein Maximum für die kleinsten Poren bei einem Durchmesser von rund $1,50 \mu \mathrm{m}$. Die Form der Verteilung deutet auf eine Normalverteilung im log-log Plot hin. Die lichtmikroskopischen Bilder zeigen ein zweites Maximum für Poren mit Durchmessern von 200 bis $600 \mu \mathrm{m}$. Dies weist auf ein Sekundärporensystem hin, das u. A. für die hohen Wassergehalte bei Sättigung verantwortlich ist, das mit der Parametrisierung der Retentionskurve nicht erfasst werden konnte. Solch ein Struktur-Porensystem findet sich laut Durner (1991) in unbearbeiteten Böden jeder Textur, somit auch bei dem untersuchten Waldboden.

Die ungesättigte Wasserleitfähigkeit beträgt $1,94 \mathrm{~cm} / \mathrm{d}$, womit der Boden als gering durchlässig (kf2) einzustufen ist. Die Korngrößenanalyse beschreibt den untersuchten Bodenhorizont als einen stark tonigen Schluff (Ut4), wobei Grobschluff die Hauptkornfraktion darstellt. Eine Berechnung der zugehörigen Porendurchmesser für würfelförmige und hexagonale Kugelpackungen ergibt Kapillarendurchmesser von 5,69 bis $17,93 \mu \mathrm{m}$. Dies korreliert sehr gut mit der bildanalytisch gemessenen Grenze zwischen struktureller und textureller Fraktalität.

Die Parametrisierung der Retentionskurve zeigt für den Ansatz nach van Genuchten (1980) eine wesentlich bessere Anpassung als nach Brooks \& Corey (1964). Um die im Labor gemessenen ungesättigten Wasserleitfähigkeiten mit der von Mualem (1976) vorgeschlagenen Funktion wiedergeben zu können, erbringt ein bimodaler Ansatz für die Retentionskurve die bessere Übereinstimmung. Die dafür berechneten van GenuchtenParameter weisen für den sättigungsnahen Bereich auf eine gröbere Textur hin als die für den sättigungsfernen Bereich.

Über die Beziehung 4-n=D kann dem Anpassungsparameter $\mathrm{n}$ der van GenuchtenFunktion eine fraktale Dimension zugeordnet werden. Der einfache van Genuchten Ansatz ergibt $\mathrm{D}=2,838$ und der doppelte für den sättigungsnahen Bereich $\mathrm{D}=2,927$ und für den sättigungsfernen $\mathrm{D}=2,746$. Besonders die Ergebnisse aus dem bimodalen Ansatz stimmen mit den bildanalytisch gemessenen Dimensionen der Porenraumgrenzlinie gut überein. Somit wird gezeigt, dass sich die bildanalytisch gemessenen D direkt mit aus der Desorptionskurve abgeleiteten fraktalen Dimensionen vergleichen lassen, wobei insbesondere der Trend zu höherer Komplexität des Porensystems für gröbere Bereiche im untersuchten Boden bestätigt wird. 



\section{Literatur}

AG Boden, 1994. Bodenkundliche Kartieranleitung, 3., verbesserte und erweiterte Auflage, Hannover.

Ahl, C., Frede, H.-G., Gäth, S., Meyer, B., 1985. Böden aus Löss im Leinetalgraben und seiner Hochflächen-Umrandung. Mitteilungen Dt. Bodenkundl. Gesellsch., 42: 359434.

Ahl, C., Niemeyer, J., 1989. The fractal dimension of the pore-volume inside soils. Z. Pflanzenernähr. Bodenk., 152:457-458.

Altemüller, H.-J., 1995. Polarisations- und Phasenkontrastmikroskopie mit dünnen Bodenschliffen. In: Babel, U., Fischer, W.R., Kaupenjohann, M., Roth, K., Stahr, K. (Hrsg.), Mikromorphologische Methoden in der Bodenkunde. Hohenheimer Bodenkundliche Hefte, 40:21-88.

Anderson, J.L., Bouma, J. 1973. Relationships between saturated hydraulic conductivity and morphometric data of an argillic horizon. Soil Sci. Soc. Am. Proc., 37:408-413.

Anderson, A.N., McBratney, A.B., Crawford, J.W., 1998. Applications of fractals to soil studies. In: Sparks, D.L. (Editor), Advances in Agronomy, Vol. 63, Academic Press, pp 1-76.

Anderson, A.N., McBratney, A.B., FitzPatrick, E.A., 1996. Soil mass, surface, and spectral fractal dimensions estimated from thin section photographs. Soil Sci. Soc. Am. J., 60:962-969.

Bässmann, H., Besslich, Ph.W., 1991. Bildverarbeitung ad oculus. Springer, 249 S.

Banerji, K., 1987. Estimation of the "true" fracture roughness parameters by iterative optimization of a reversed sigmoidal fractal model. Acta Stereol., 6/III:815-820.

Bartoli, F., Philippy, R., Doirisse, M., Niquet, S., Dubuit, M., 1991. Structure and selfsimilarity in silty and sandy soils: The fractal approach. J. Soil Sci., 42:167-185.

Bartoli, F., Bird, N.R., Gomendy, V., Vivier, H., Niquet, S., 1999. The relation between silty soil structures and their mercury porosimetry curve counterparts: fractals and percolation. Europ. J. Soil Sci., 50:9-22.

Baveye, P., Boast, C.W., 1998. Fractal geometry, fragmentation processes and the physics of scale invariance: an introduction. In: Baveye, P., Parlange, J.Y., Stewart, B.A. (Editors), Fractals in Soil Science. CRC Press, Boca Raton, pp. 1-54.

Baveye, P., Boast, C.W., Ogawa, S., Parlange, J.Y., Steenhuis, T., 1998. Influence of image resolution and thresholding on the apparent mass fractal characteristics of preferential flow patterns in field soils. Water Resources Research, 34:2783-2796.

Becher, H.H., 1971. Ein Verfahren zur Messung der ungesättigten Wasserleitfähigkeit. Z. Pflanzenernähr. Bodenk., 128:1-12.

Bird, N.R.A., Bartoli, F., Dexter, A.R., 1996. Water retention models for fractal soil structures. Europ. J. Soil Sci., 47:1-6.

Bird, N.R.A., Dexter, A.R., 1997. Simulation of soil water retention using random fractal networks. Europ. J. Soil Sci., 48:633-641.

Brooks, R.H., Corey, A.T., 1964. Hydraulic properties of porous media. In: Hydrology Paper 3:22-27, Colorado State University, Fort Collins, Colorado. 
Bullock, P., Federoff, N., Jongerius, A., Stoops, G., Tursina, T., 1985. Handbook for Soil Thin Section Description. Waine Research Publications, Wolverhampton, UK: 152 $\mathrm{S}$.

Burdine, N.T., 1953. Relative permeability calculation from size distribution data. Trans. AIME, 198:71-78.

Carsel, R.F., Parrish, R.S., 1988. Developing joint probability distributions of soil water retention characteristics. Water Resources Research 24: 755-769.

Celis, R., Cornejo, J., Hermosin, M.C., 1996. Surface fractal dimensions of synthetic clayhydrous iron oxide associations from nitrogen adsorption isotherms and mercury porosimetry. Clay minerals, 31:355-363.

Comegna, V., Damiani, P., Sommella, A., 1998. Use of a fractal model for determining soil water retention curves. Geoderma 85:307-323.

Crawford, J.W., 1994. The relationship between structure and the hydraulic conductivity of soil. Europ. J. Soil Sci., 45:493-502.

Crawford, J. W.; Ritz, K.; Young, I.M., 1993a. Quantification of fungal morphology, gaseous transport and microbial dynamics in soil: an integrated framework utilising fractal geometry. Geoderma, 56: 157-172.

Crawford, J. W.; Sleeman, B. D.; Young, I. M., 1993b. On the relation between numbersize distributions and the fractal dimension of aggregates. Journal of Soil Science, 44: 555-565.

Crawford, J.W., Verall, S., Young, I.M., 1997. The origin and loss of fractal scaling in simulated soil aggregates. Europ. J. Soil Sci., 48:643-650.

Crawford, J.W., Matsui, N., Young, I.M., 1995. The relation between the moisture-release curve and the structure of soil. Europ. J. Soil Sci., 46:369-375.

Crawford, J.W., Pachepsky, Ya.A., Rawls, W.J., 1999. Integrating processes in soils using fractal models. Editorial of Geoderma 88, Special Issue: Fractals in Soil Science, pp. 103-107.

Cross, S.S., 1994. The application of fractal geometric analysis to microscopic images. Micron, 25:101-113.

Dathe, A., Eins, S., Niemeyer, J., Gerold, G., 2001. The surface fractal dimension of the soil-pore system as measured by image analysis. Geoderma 103/1-2:203-229.

Dathe, A., Walter, N., Eins, S., Gerold, G., 1998. Bildanalytische Charakterisierung des Porenraums im Boden im Vergleich mit hydraulischen Prozessparametern. Mitteilungen Dt. Bodenkundl. Gesellsch. 88:359-362

Diekkrüger, B., 1992. Standort- und Gebietsmodelle zur Simulation der Wasserbewegung in Agrarökosystemen. Landschaftsökologie und Umweltforschung, Institut für Geographie und Geoökologie der TU Braunschweig, Heft 19, 169 S.

Dünger, O., Durner, W. Papritz, A., 1995. Veränderungen der Porenstruktur eines Bodens durch die Messung der Retentionskurve. Mitteilungen Dt. Bodenkundl. Gesellsch., 76:95-98.

Durner, W., 1991: Vorhersage der hydraulischen Leitfähigkeit strukturierter Böden. Bayreuther Bodenkundliche Berichte, Bd. 20.

Ehlers, W., Wendroth, O., de Mol, F., 1995. Characterising pore organisation by soil physical parameters. In: Hartge, K.H., Stewart, B.A. (Editors.). Soil Structure: Its Development and Function. Advances in Soil Science, pp. 257-275. 
Eins, S., 1994. An image analysis approach to the measurement of fractal dimensions: application to glial cells. J. Brain Res. 35: 129-130.

Eins, S., 1995. An improved dilation method for the measurement of fractal dimension. Acta Stereol., 14:169-178.

Eins, S., 1998. Special approaches of image analysis for the measurement of fractal dimension. In: Losa, G.A., Merlini, D., Nonnenmacher, T.F., Weibel, E.R. (Editors), Fractals in Biology and Medicine II. Birkhäuser, Basel, pp. 86-96.

Eins, S., Stiller, K.J., 1995. Automatische Bildanalyse. In: Robenek, H. (Hrsg.), Mikroskopie in Forschung und Praxis, GIT Verlag: 325-392.

Falconer, K.J., 1990. Fractal Geometry. Mathematical Foundations and Applications. John Wiley \& Sons. Ltd.. Chichester.

Feder, J., 1988. Fractals. Plenum Press, New York.

FitzPatrick, E.A., 1993. Soil Microscopy and Micromorphology. Wiley: 304 S.

Flook, A.G., 1978. The use of dilation logic on the Quantimet to achieve fractal dimension characterisation of textured and structured profiles. Powder Technol., 21:295-298.

Frede, H.G., Meyer, B., 1983. Bestimmung der Dichte von Boden-Aggregaten durch Messung des Auftriebs. Z. Pflanzenernähr. Bodenkd., 146:365-368.

Friedrichs, O., 1999. Vergleichende bodenphysikalische Untersuchungen an Stechzylinderproben und Einzelaggregaten. Unveröffentlichte Diplomarbeit Univ. Göttingen, $118 \mathrm{~S}$.

Gerlach, D., 1995. Lichtmikroskopie. In: Robenek, H. (Hrsg.), Mikroskopie in Forschung und Praxis, GIT Verlag: 399 S.

Germann, P., 2001. Macropore Modelling: A Hydromechanical Approach. In: Anderson, M.G., Bates, P.D. (Editors), Model Validation: Perspectives in Hydrological Science. Wiley, Chichester. In Press: 320 p.

Germann, P., Di Pietro, L., 1999. Scales and dimensions of momentum dissipation during flow in soils. Water Resources Research 35, No. 5:1443-1454.

Giménez, D., Allmaras, R.R., Nater, E.A., Huggins, D.R., 1997. Fractal dimensions for volume and surface of interaggregate pores - scale effects. Geoderma, 77:19-38.

Glasow, A., 2000. Stoffliche Veränderungen im Mikro- und Submikrobereich von Mineralkörnern und Bildung neuer Phasen bei Verwitterung und Bodenbildung in Löß. Dissertation Universität Göttingen: $108 \mathrm{~S}$.

Göttlein, A., Hell, U. Blasek, R., 1996. A system for microscale tensiometry and lysimetry Geoderma, 69:147-156.

Gouyet, J.-F. 1996. Physics and Fractal Structures. Masson, Springer-Verlag: 234 S.

Gunzelmann, M., 1990. Die Quantifizierung und Simulation des Wasserhaushalts von Einzelaggregaten und strukturierten Gesamtböden unter besonderer Berücksichtigung der Wasserspannungs/Wasserleitfähigkeitsbeziehung von Einzelaggregaten. Bayreuther Bodenk. Ber., Bd. 15: 130 S.

Hamblin, M.G., Stachowiak, W.G., 1994. Measurement of fractal surface profiles obtained from scanning electron and laser scanning microscope images and contact profile meter. J. Comput. Assist. Microsc., 6:181-194.

Hartge, K.H., 1966. Ein Haubenpermeameter zum schnellen Durchmessen zahlreicher Stechzylinderproben. Z. f. Kulturtechnik u. Flurber., 7:155-163. 
Heijs, A.W.J., de Lange, J., 1997. Determination of pore networks and water content distributions from 3-D computed tomography images of a clay soil. Bioimaging, 5:194-204.

Junkersfield, L.E., 1995. Dynamik der Wasserhaushaltsparameter von Aggregaten und Gesamtboden einer pseudovergleyten Parabraunerde aus Geschiebemergel. Schriftenreihe des Instituts für Pflanzenernährung und Bodenkunde der Universität Kiel, Nr. 31, 108 S.

Junkersfield, L. Horn, R., 1997. Über die räumliche und zeitliche Variabilität scheinbar fixer Wasserhaushaltsgrößen am Beispiel von Bodenaggregaten. Z. Pflanzenernähr. Bodenkunde, 160:179-186.

Kaye, B.H., 1989. Image analysis techniques for characterizing fractal structures. In: Avnir, D. (ed.): The fractal approach to heterogeneous chemistry. Chichester, J. Wiley \& Sons: 55-66.

Klute, A. 1986. Water retention: Laboratory methods. In: Klute, A. (Editor), Methods of Soils Analysis, Part 1, Physical and Mineralogical Methods, Second Edition. Agronomy Series 9, American Society of Agronomy, Madison, Wisconsin:635-662.

Korvin, G., 1992. Fractal models in the earth sciences. Amsterdam, Elsevier: 396 S.

Krahmer, U., 1987. EDV-gestütztes Meß- und Auswerteverfahren zur Bestimmung der ungesättigten Wasserleitfähigkeit nach der Verdunstungsmethode. Z. Pflanzenernähr. Bodenk., 150:392-394.

Kruhl, J.H. (ed.), 1994. Fractals and dynamic systems in the earth sciences. Berlin, Springer- Verlag: $421 \mathrm{~S}$.

Kuntze, H., Roeschmann, G. Schwerdtfeger, G. (1994): Bodenkunde. 5. Aufl., Ulmer, Stuttgart. $424 \mathrm{~S}$.

Mandelbrot, B.B., 1967. How long is the coast of Britain? Statistical self similarity and fractional dimension. Science, 156:636-638.

Mandelbrot, B.B., 1983. The Fractal Geometry of Nature. W.H. Freeman \& Co., New York.

Mandelbrot, B.B., Passoja, D.E., Paullay, A.J., 1984. Fractal character of fracture surfaces of metals. Nature Vol. 308: 721-722.

Meyberg; K., Vachenauer, P, 1993. Höhere Mathematik. 1. Differential- und Intergralrechnung, Vektor- und Matrizenrechnung. Springer-Verlag, $529 \mathrm{~S}$.

Moreau, E., Velde, B., Terribile, F., 1999. Comparison of 2D and 3D images of fractures in a Vertisol. Geoderma 92: 55-72.

Mualem, Y., 1976. A new model for predicting the hydraulic conductivity of unsaturated porous media. Water Resources Research 12: 513-522.

Neimark, A.V., 1989. Multiscale percolation systems. Sov. Phys.-JETP, 69: 786-791.

Niemeyer, J., Machulla, G., 1999. Description of soil pore systems accessible for water by fractal dimensions. Physica A, 266:203-208.

Ogawa, S., Baveye, P., Boast, C.W., Parlange, J.Y., Steenhuis, T., 1999. Surface fractal characteristics of preferential flow patterns in field soils: evaluation and effect of image processing. Geoderma, 88:109-136.

Opara-Nadi, O.A., 1979. A comparision of some methods for determing the hydraulic conductivity of unsaturated soils in the low suction range. Göttinger Bodenkundliche Berichte 57, $104 \mathrm{~S}$. 
Orford, J.D., Whalley, W.B., 1983. The use of the fractal dimension to quantify the morphology of irregular-shaped particles. Sedimentology, 30:665-668.

Pachepsky, Ya., Yakovchenko, V., Rabenhorst, M.C., Pooley, C., Sikora, L.J., 1996. Fractal parameters of pore surfaces as derived from micromorphological data: effect of long-term management practices. Geoderma, 74:305-319.

Pape, H., 1996. Fractal geometry and fractal „music of the earth“ (according to a term of H. Cloos): Zbl. Geol. Paläont., 9/10:969-980.

Pape, H., Riepe, L., Schopper, J.R., 1987. Theory of self-similar network structures in sedimentary and igneous rocks and their investigation with microscopical and physical methods. Journal of microscopy, 148:121-147.

Perfect, E., Kay, B.D., 1991. Fractal theory applied to soil aggregation. Soil Sci. Soc. Am. J., 55:1552-1558.

Perfect, E., McLaughlin, N.B., Kay, B.D., Topp, G.C., 1996. An improved fractal equation for the soil water retention curve. Water Resources Research, 32:281-287.

Perrier, E., Bird, N., Rieu, M., 1999. Generalizing the fractal model of soil structure: the pore-solid fractal approach. Geoderma, 88:137-164.

Perrier, E., Mullon, C., Rieu, M., 1995. Computer construction of fractal soil structures: Simulation of their hydraulic and shrinkage properties. Water Resources Research, 31:2927-2943.

Perrier, E., Rieu, M., Sposito, G., de Marsily, G., 1996. Models of the water retention curve for soils with a fractal pore size distribution. Water Resources Research, 32:3025-3031.

Plagge, R., 1991. Bestimmung der ungesättigten Leitfähigkeit im Boden. Bodenökologie und Bodengenese, Heft 3, Berlin.

Press, W.H., Teukolsky, S.A., Vetterling, W.T., Flannery, W.P., 1992. Numerical Recipes in C. The Art of Scientific Computing. Cambridge University Press, Cambridge, second edition.

Protz, R., VandenBygaart, A.J., 1998. Towards systematic image analysis in the study of soil micromorphology. Internet Publikation. Science of Soils, 3:4.

Reichelt, R. 1995. Rasterelektroenenmikroskopie und Röntgenmikroanalyse. In: Robenek, H. (Hrsg.), Mikroskopie in Forschung und Praxis, GIT Verlag: 399 S.

Richardson, L.F., 1961. The problem of contiguity: an appendix to statistics of deadly quarrels. Gen. Sys. Yearb., 6:139-187.

Rieu, M., Sposito, G., 1991a. Fractal fragmentation, soil porosity, and soil water properties:

I. Theory. Soil Sci. Soc. Am. J., 55:1231-1238.

Rieu, M., Sposito, G., 1991b. Fractal fragmentation, soil porosity, and soil water properties: II. Applications. Soil Sci. Soc. Am. J., 55:1239-1244.

Rieu, M., Sposito, G., 1991c. Relation pression capillaire-teneur en eau dans les milieux poreux fragmentés et identification du caractère fractal de la structure des sols. C. R. acad. sci. Paris, Sèrie II, 312: 1483-1489.

Rigaut, J.P., Berggren, P., Robertson, B., 1983. Resolution-dependence of stereological estimations: interpretation with a new fractal concept of automatic image analyserobtained results on lung sections. Acta Stereol., 2 (suppl 1):121-124. 
Rohdenburg, H., Meyer, B., 1979. Zur Feinstratigraphie und Paläopedologie des Jungpleistozäns nach Untersuchungen an südniedersächsischen und nordhessischen Lößprofilen. Landschaftsgenese und Landschaftsökologie, 3:1-89.

Roth, K. (1996). Soil Physics Lecture Notes. University of Hohenheim, Institute of Soil Science, Stuttgart, Germany.

Schachtschabel, P., Blume, H.-P., Brümmer, G., Hartge, K.-H., Schwertmann, U., 1989. Scheffer/Schachtschabel: Lehrbuch der Bodenkunde. Enke, Stuttgart. 491 S.

Schindler, U., 1980. Ein Schnellverfahren zur Messung der Wasserleitfähigkeit im teilgesättigten Boden an Stechzylinderproben. Archiv für Acker- und Pflanzenbau und Bodenkunde, 24:1-7.

Schlichting, E., Blume, H.P., Stahr, K., 1995. Bodenkundliches Praktikum. 2. Aufl., Blackwell, Berlin. $295 \mathrm{~S}$.

Schmidt, P.F., 1994. Praxis der Rasterelektronenmikroskopie und Mikrobereichsanalyse. Kontakt und Studium, 444, expert-Verlag, Renningen-Malmsheim: $810 \mathrm{~S}$.

Schroeder, M., 1991. Fractals, Chaos, Power Laws. Freeman, New York: 429 S.

Schultze, B. Durner, W., 1997. Bestimmung der hydraulischen Eigenschaften von Bodenproben durch inverse Simulation instationärer Fließexperimente: Dynamische Effekte. Mitteillungen. Dt. Bodenkundl. Gesellsch., 85, I:163-166.

Schultze, B., Zurmühl, T., Durner, W., 1995. Ein Vergleich von Onestep-, Multistep- und Kontinuierlichen Gradientenverfahren zur Bestimmung hydraulischer Funktionen von Bodensäulen. Mitteilgn. Dtsch. Bodenkundl. Gesellsch., 76, 157-160.

Sernetz, M., 2000. Die fraktale Geometrie des Lebendigen. Spektrum der Wissenschaft 7, 2000:72-79.

Serra, J. 1982. Image Analysis and Mathematical Morphology. Academic Press, London: $610 \mathrm{~S}$.

Stacheder, M., 1996. Die Time Domain Reflektometry in der Geotechnik. Schriftenreihe Angewandte Geologie Karlsruhe, 40, Universität Karlsruhe: 170 S.

Stahel, W. A., 2000. Statistische Datenanalyse. Eine Einführung für Naturwissenschaftler. Dritte Auflage, Vieweg Braunschweig/Wiesbaden, 379 S.

Stolte, J., Freijer, J.I., Bouten, W. u. a., 1994. Comparison of six methods to determine unsaturated soil hydraulic conductivity. Soil Sci. Soc. Am. J., 58: 1596-1603.

Tietje, O., Tapkenhinrichs, M., 1993. Evaluations of Pedo-Transfer Functions. Soil Sci. Soc. Am. J., 57:1088-1095.

Tyler, S.W., Wheatcraft, S.W., 1989. Application of fractal mathematics to soil water retention estimation. Soil Sci. Soc. Am. J., 53:987-996.

Universitäts-Rechenzentrum Trier (Hrsg.), 1998. Statistische Datenanalyse mit Excel: 29 S.

van Genuchten, M.Th., 1980. A closed-form equation for predicting the hydraulic conductivity of unsaturated soils. Soil Sci. Soc. Am. J. 44: 892-898.

van Genuchten, M.Th., Leij, F.J., Yates, S.R., 1991. The RETC Code for Quantifying the Hydraulic Functions of Unsaturated Soils, Version 1.0. EPA Report 600/2-91/065, U.S. Salinity Laboratory, USDA, ARS, Riverside, California.

Vogel, H.-J., 1996. Die Kontinuität des Porenraums in Abhängigkeit der Porengröße - eine morphologische Materialfunktion. Mitteilungen Dt. Bodenkundl. Gesellsch., 80:291294. 
Vogel, H.-J., 2000: A numerical experiment on pore size, pore connectivity, water retention, permeability, and solute transport using network models. Europ. J. Soil Sci., 51:99-105.

Vogel, H.-J., Kretzschmar, A., 1996. Topological characterization of pore space in soil sample preparation and digital image-processing. Geoderma, 73:23-38.

Vogel, H.-J., Roth, K., 1997. Modellierung der Porenstruktur aus morphologischen Daten zur Schätzung effektiver Materialfunktionen. Mitteilungen Dt. Bodenkundl. Gesellsch., 85, I:179-182.

Walker, A., 1994. ModelMaker. Cherwell Scientific Publishing, Oxford.

Weibel, E.R., 1991. Fractal geometry: a design for living organisms. American Journal of Physiology, 261:361-369.

Witten, T.A., Sander, L.M., 1981. Diffusion-limited aggregation, a kinetic critical phenomenon. Physical Review Letters 47/19: 1400-1403. 

Anhang 


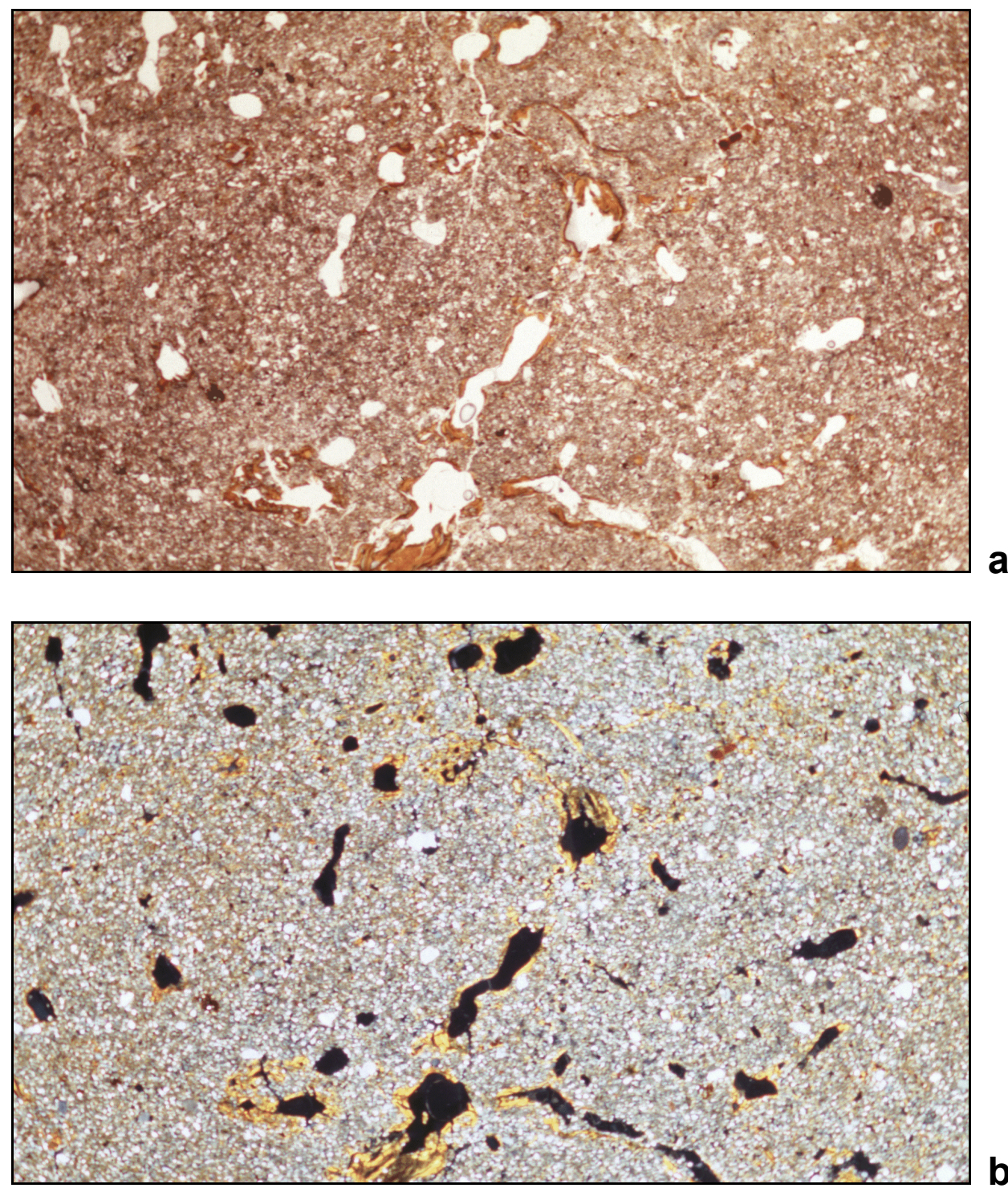

b

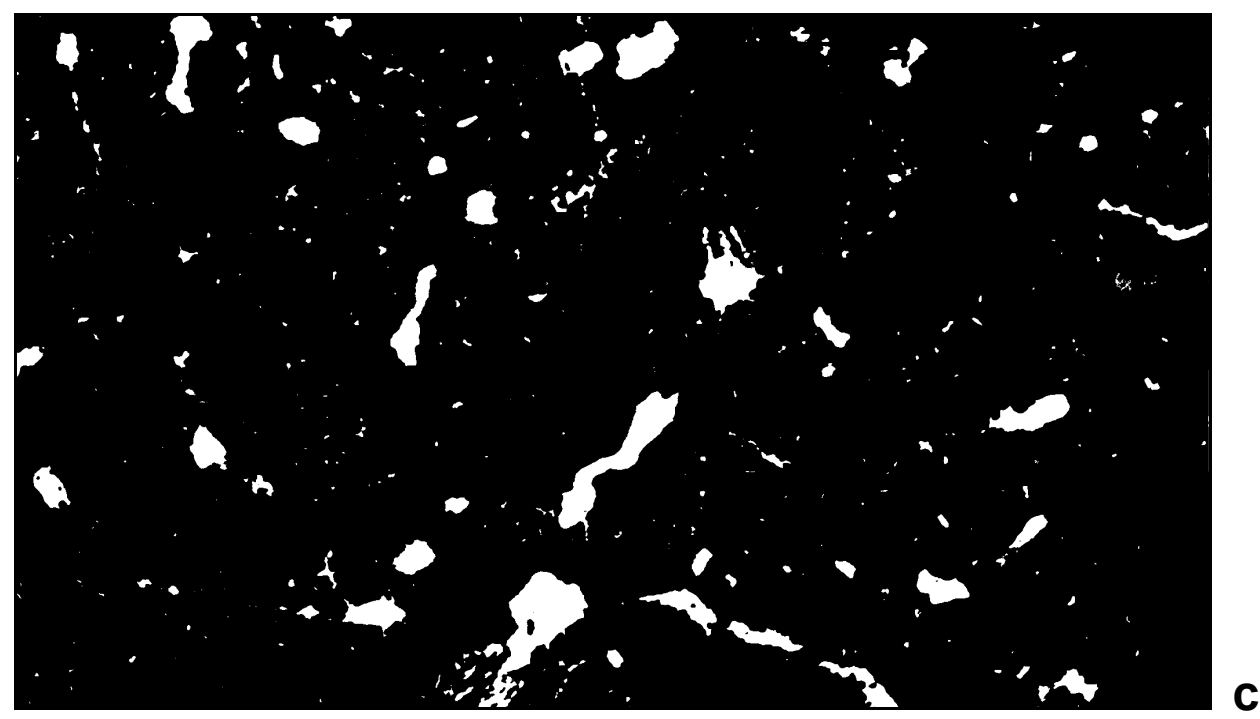

Abb. A1: Durchlichtaufnahmen des Dünnschliffes Wild 8 (horizontale Ausrichtung) mit dem Photomakroskop (Wild), Objektiv 6.3. (a) im Hellfeld, (b) mit polarisiertem Licht, (c) nach Grauwertsegmentierung erhaltenes Binärbild von (b). Die Bildgröße beträgt rund 16,0 x 9,3 mm. 

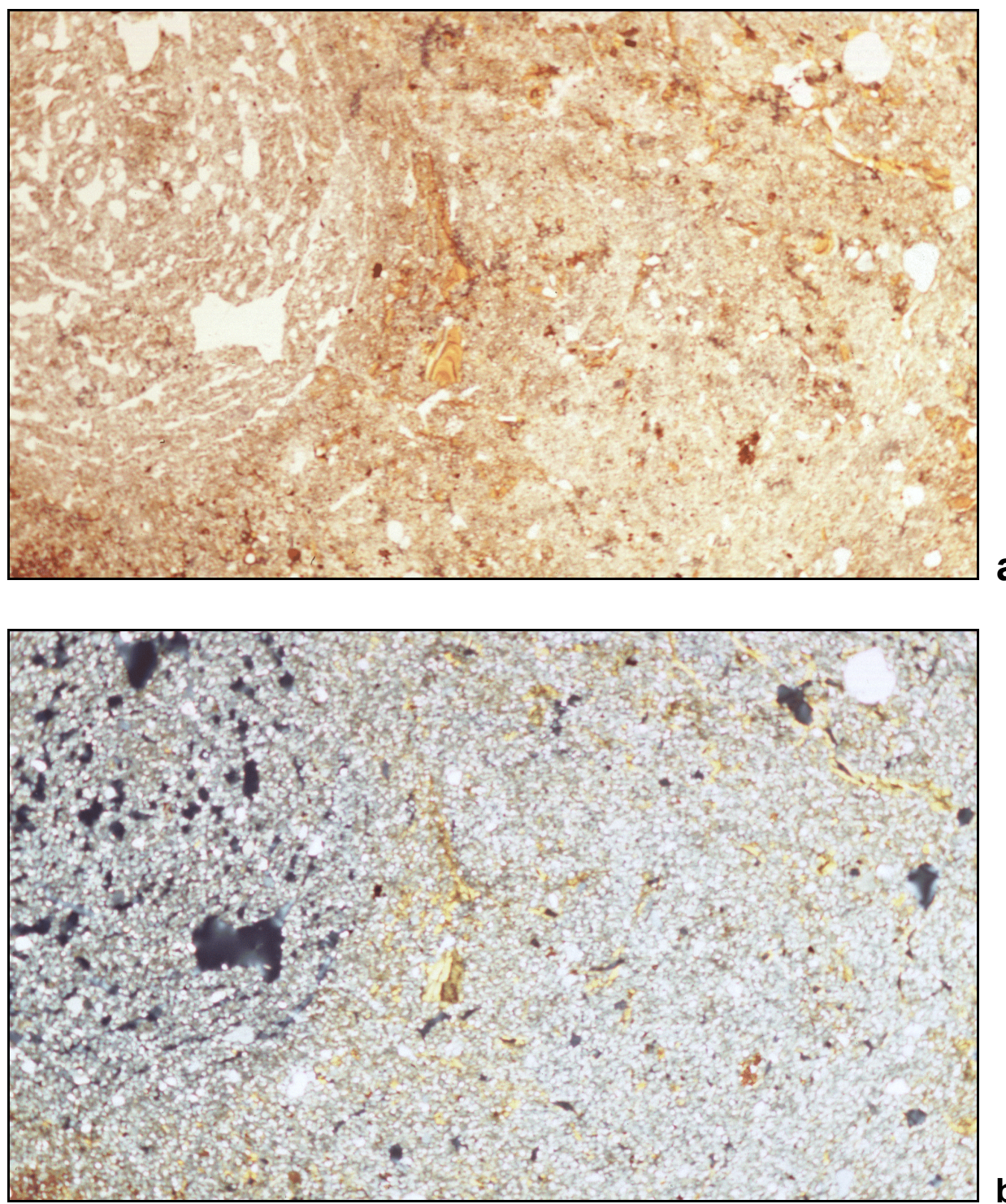

b

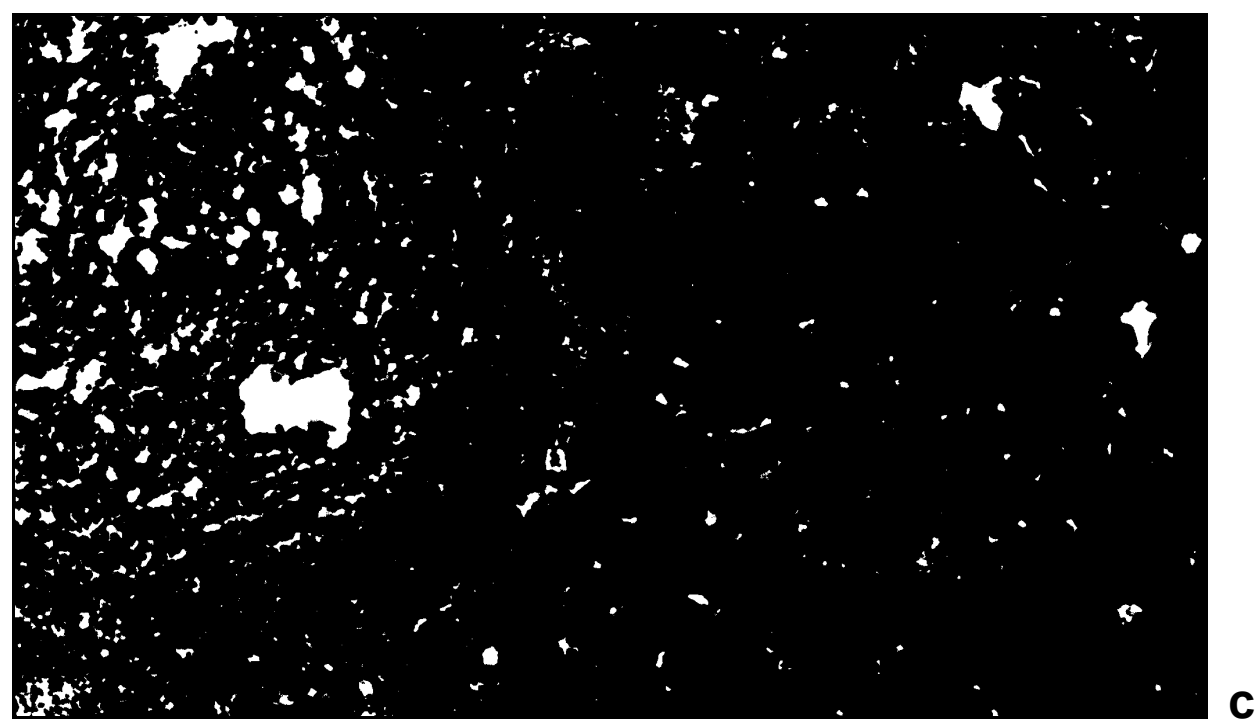

Abb. A2: Durchlichtaufnahmen des Dünnschliffes Wild 9 (horizontale Ausrichtung) mit dem Photomakroskop (Wild), Objektiv 6.3. (a) im Hellfeld, (b) mit polarisiertem Licht, (c) nach Grauwertsegmentierung erhaltenes Binärbild von (b). Die Bildgröße beträgt rund 16,0 x 9,3 mm. 

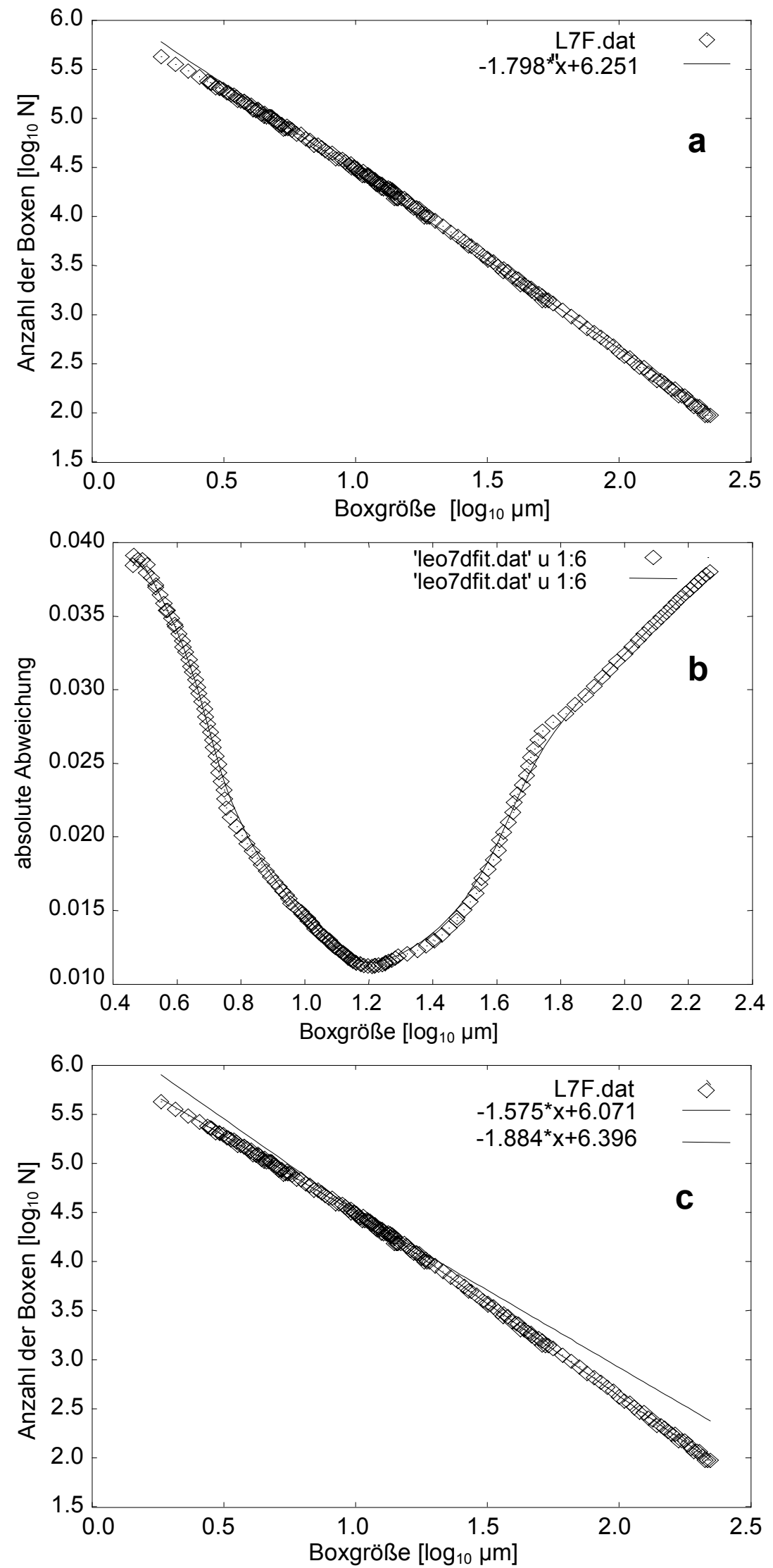

Abb. A.3: Ergebnisse des robusten Schätzverfahrens (lokale M-Schätzer) für alle Vergrößerungsstufen des Schliffes Leo7. (a) Anpassung einer Regressionsgeraden für die nach der Methode B gemittelten Daten des Boxcounting-Verfahrens; (b) Darstellung der absoluten Abweichungen für die Anpassung von zwei Geraden für Boxcounting; (c) die gleichen Daten wie in (a), die Anpassung von zwei Geraden erfolgte für die gering7ste absolute Abweichung; 

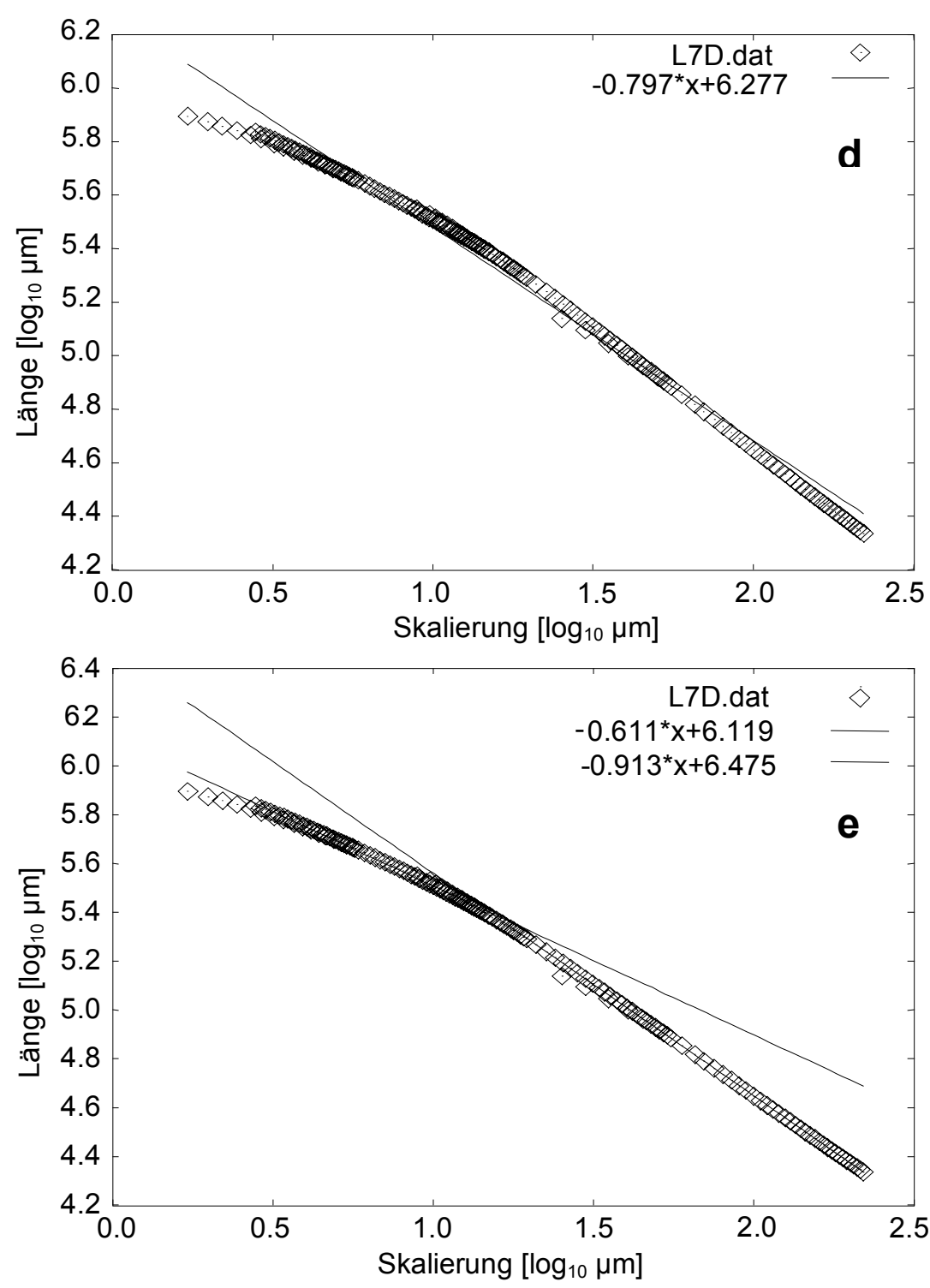

Abb. A.3: Ergebnisse des robusten Schätzverfahrens (lokale M-Schätzer) für alle Vergrößerungsstufen des Schliffes Leo7. (d) Anpassung einer Geraden für die nach der Methode B gemittelten Daten des Dilatationsverfahrens und (e) Anpassung von zwei Geraden für die Daten von (d), die Optimierung erfolgte ebenfalls durch die Minimierung der absoluten Abweichung. 

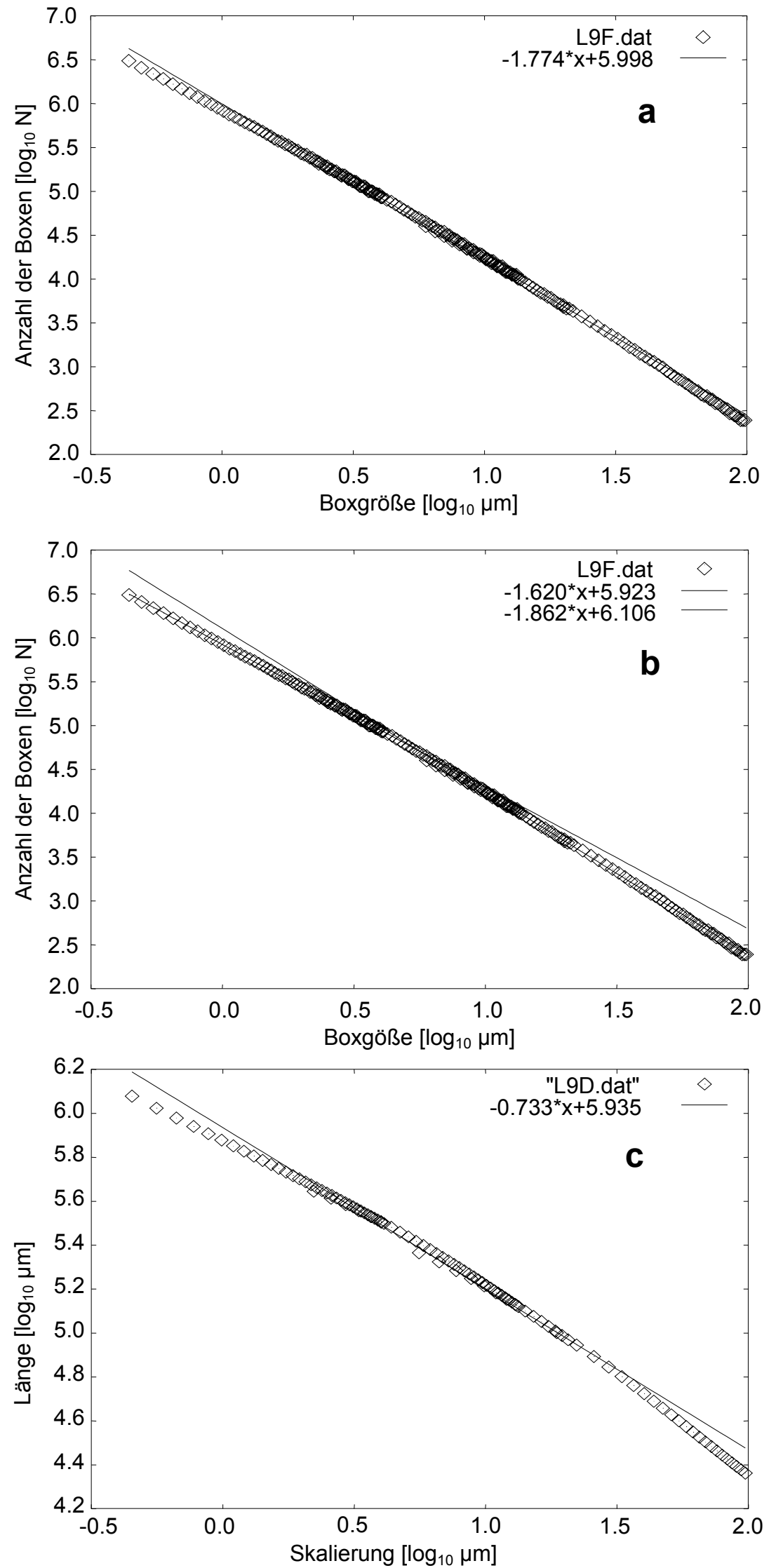

Abb. A.4: Ergebnisse des robusten Schätzverfahrens (lokale M-Schätzer) für alle Vergrößerungsstufen des Schliffes Leo9. (a) Anpassung einer Regressionsgeraden für die nach der Methode B gemittelten Daten des Boxcounting-Verfahrens; (b) die gleichen Daten wie in (a), die Anpassung von zwei Geraden erfolgte für die geringste absolute Abweichung; (c) Anpassung einer Geraden für die nach der Methode B gemittelten Daten des Dilatationsverfahrens; 

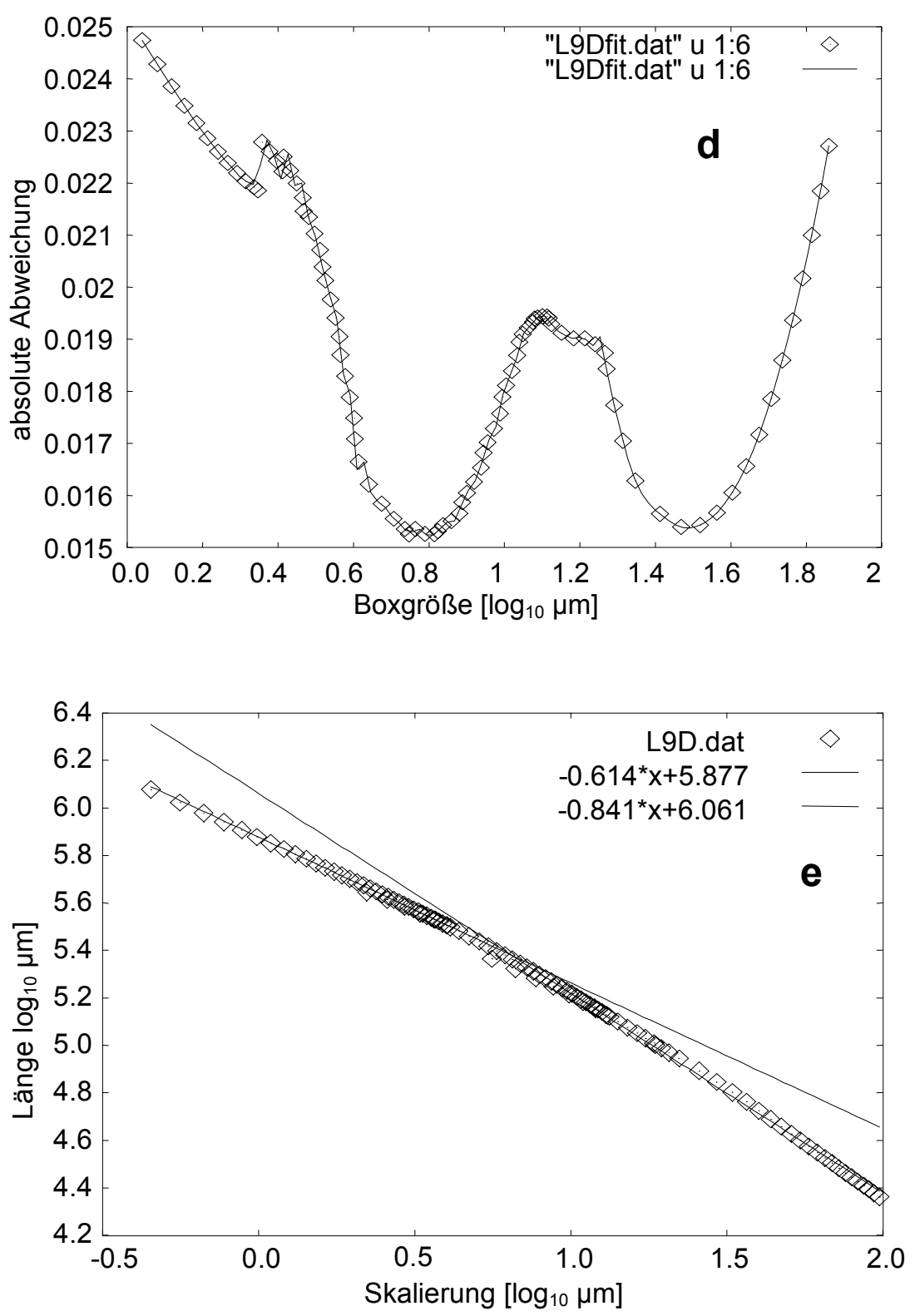

Abb. A.4: Ergebnisse des robusten Schätzverfahrens (lokale M-Schätzer) für alle Vergrößerungsstufen des Schliffes Le09. (d) Darstellung der absoluten Abweichungen für die Anpassung von zwei Geraden für Dilatation und (e) Anpassung von zwei Geraden für die Daten von (c), die Optimierung erfolgte durch die Minimierung der absoluten Abweichung. 

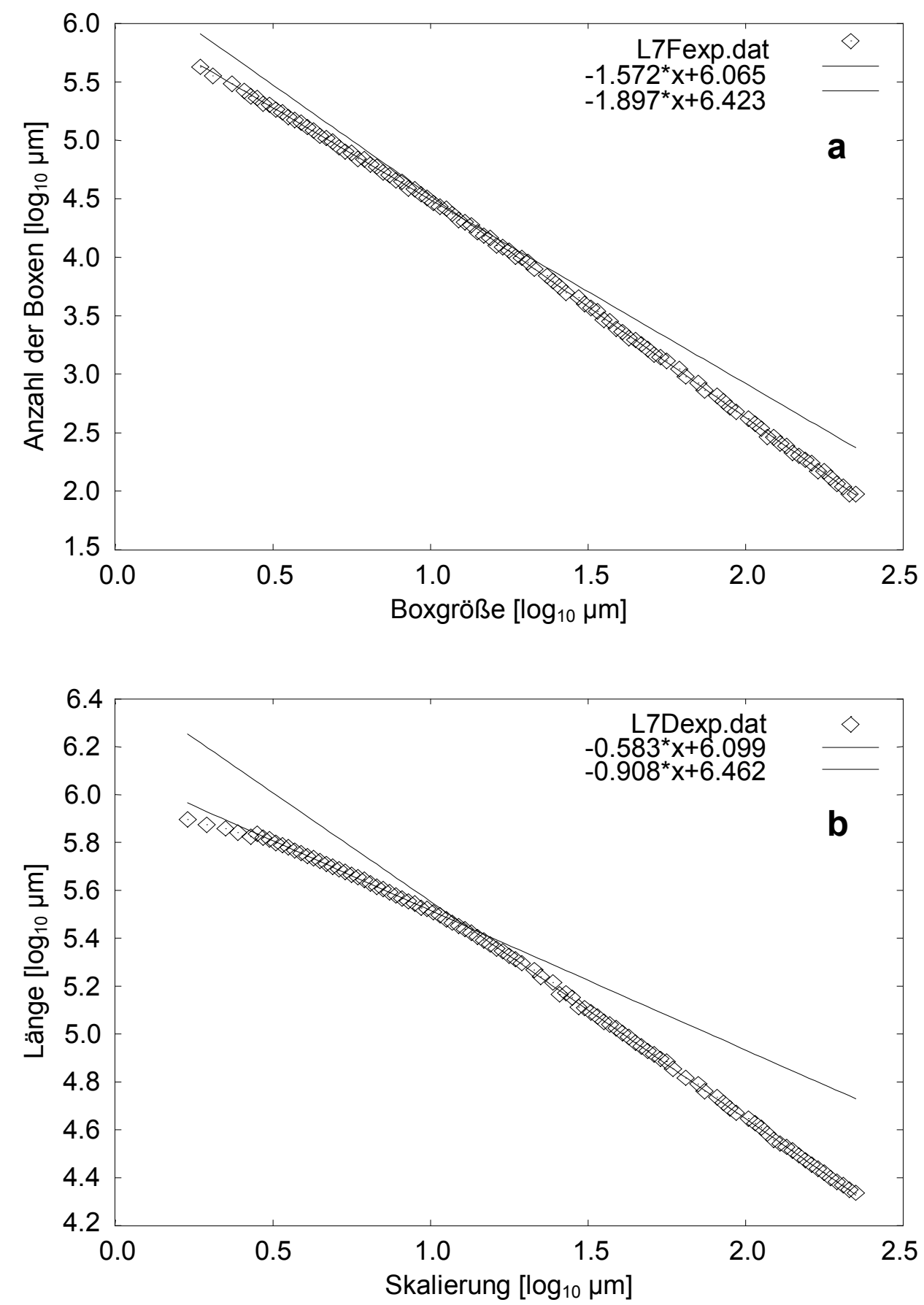

Abb. A.5: Alle Vergrößerungsstufen des Schliffes Leo7 unter Verwendung einer exponentiellen Schrittweite des Strukturelements. Die Rohdaten wurden gemittelt (Methode B) und bezüglich des yAchsenabschnitts angepasst; (a) zeigt die Anpassung von zwei Geraden für die mit Boxcounting erhobenen Messdaten und (b) zeigt die Anpassung für die mit Dilatation erhobenen Messdaten. 

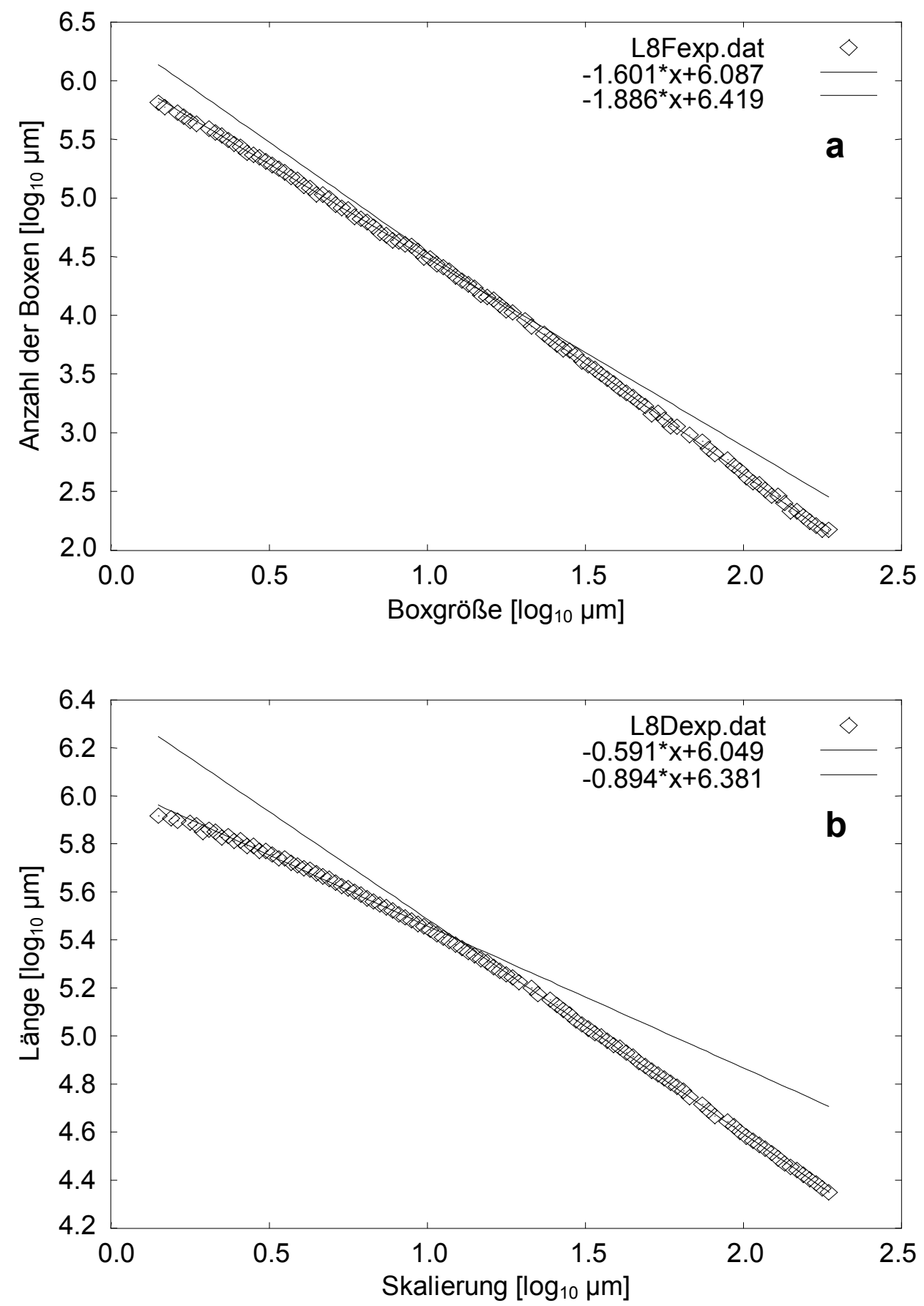

Abb. A.6: Alle Vergrößerungsstufen des Schliffes Leo8 unter Verwendung einer exponentiellen Schrittweite des Strukturelements. Die Rohdaten wurden gemittelt (Methode B) und bezüglich des yAchsenabschnitts angepasst; (a) zeigt die Anpassung von zwei Geraden für die mit Boxcounting erhobenen Messdaten und (b) zeigt die Anpassung für die mit Dilatation erhobenen Messdaten. 

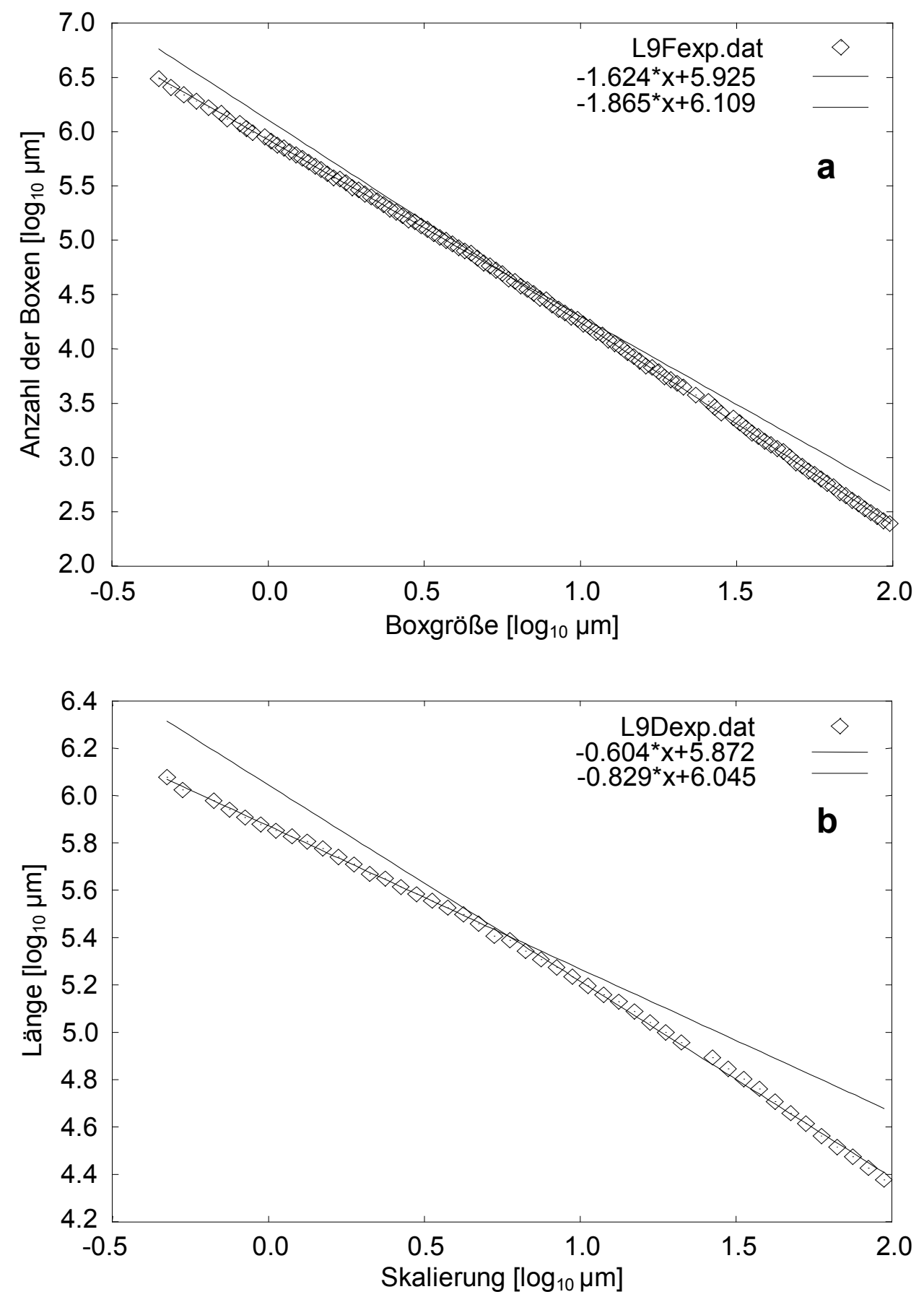

Abb. A.7: Alle Vergrößerungsstufen des Schliffes Leo9 unter Verwendung einer exponentiellen Schrittweite des Strukturelements. Die Rohdaten wurden gemittelt (Methode B) und bezüglich des yAchsenabschnitts angepasst; (a) zeigt die Anpassung von zwei Geraden für die mit Boxcounting erhobenen Messdaten und (b) zeigt die Anpassung für die mit Dilatation erhobenen Messdaten. 

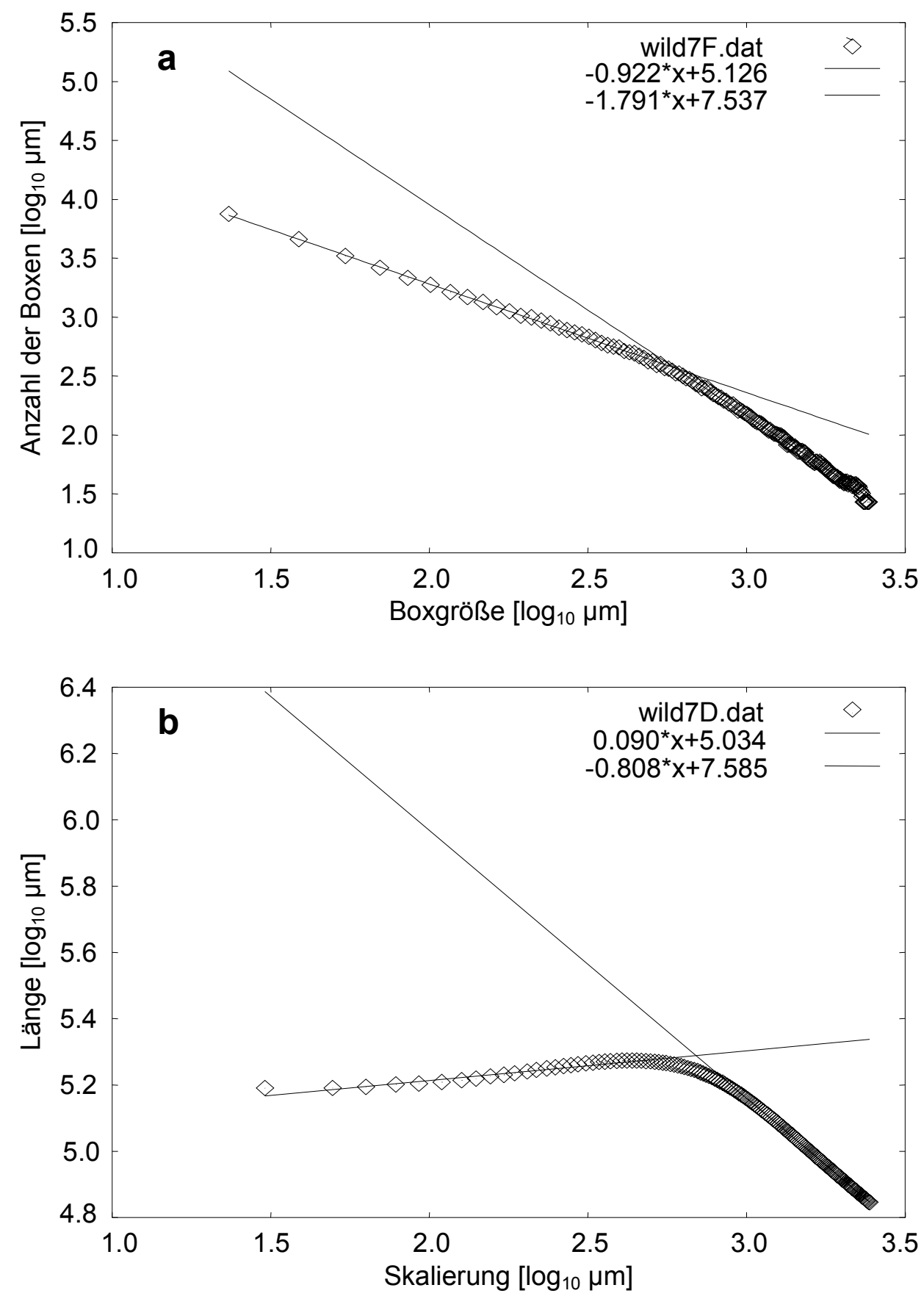

Abb. A.8: Anpassung von zwei Geraden mit dem robusten Schätzverfahren (lokale M-Schätzer) für das mit dem Wild-Photomakroskop aufgenommene Einzelbild des für die Serie Leo7 verwendeten Dünnschliffs. (a) Mit Boxcounting und (b) mit Dilatation erhobene Messdaten. 

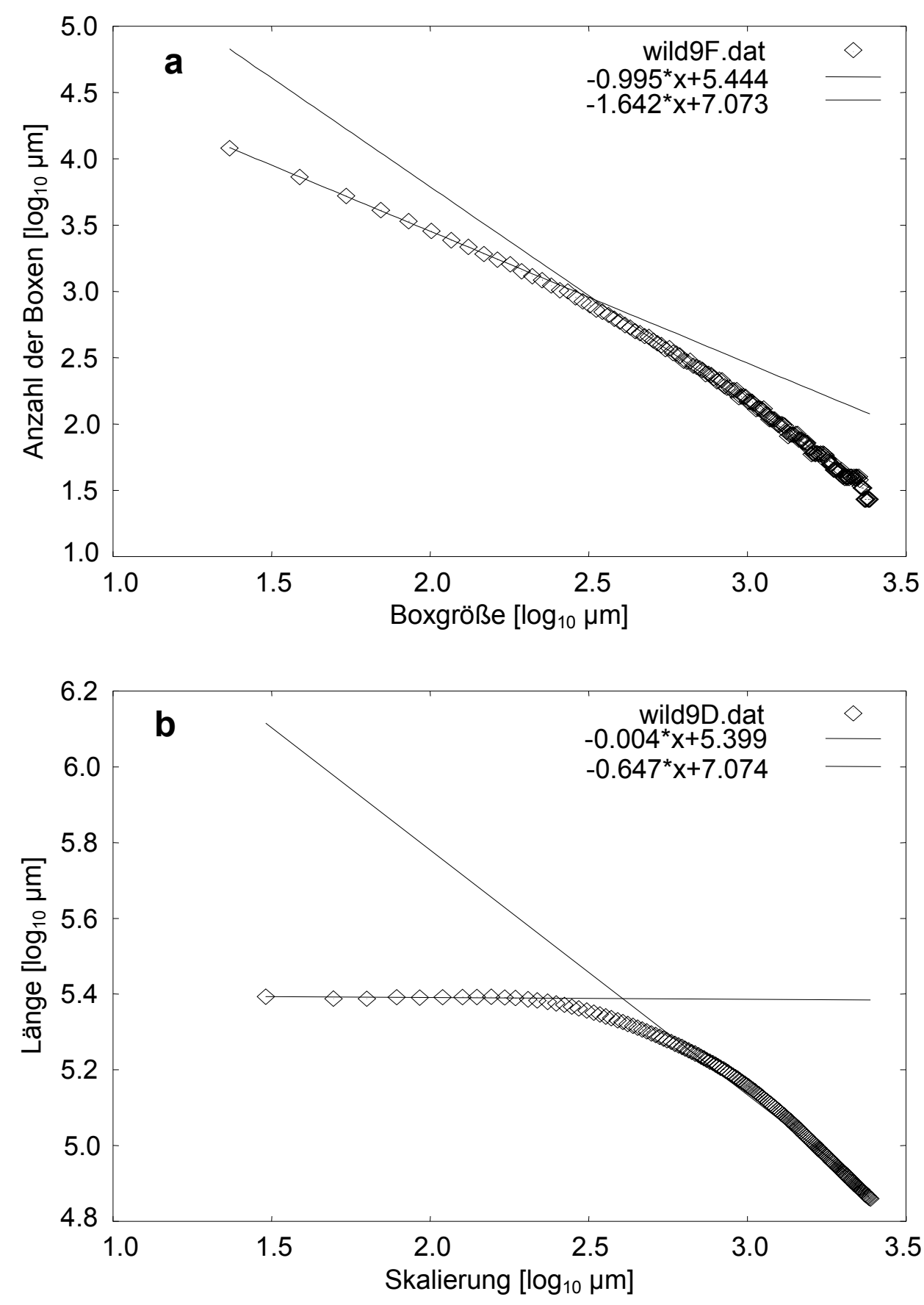

Abb. A.9: Anpassung von zwei Geraden mit dem robusten Schätzverfahren (lokale M-Schätzer) für das mit dem Wild-Photomakroskop aufgenommene Einzelbild des für die Serie Leo9 verwendeten Dünnschliffs. (a) Mit Boxcounting und (b) mit Dilatation erhobene Messdaten. 

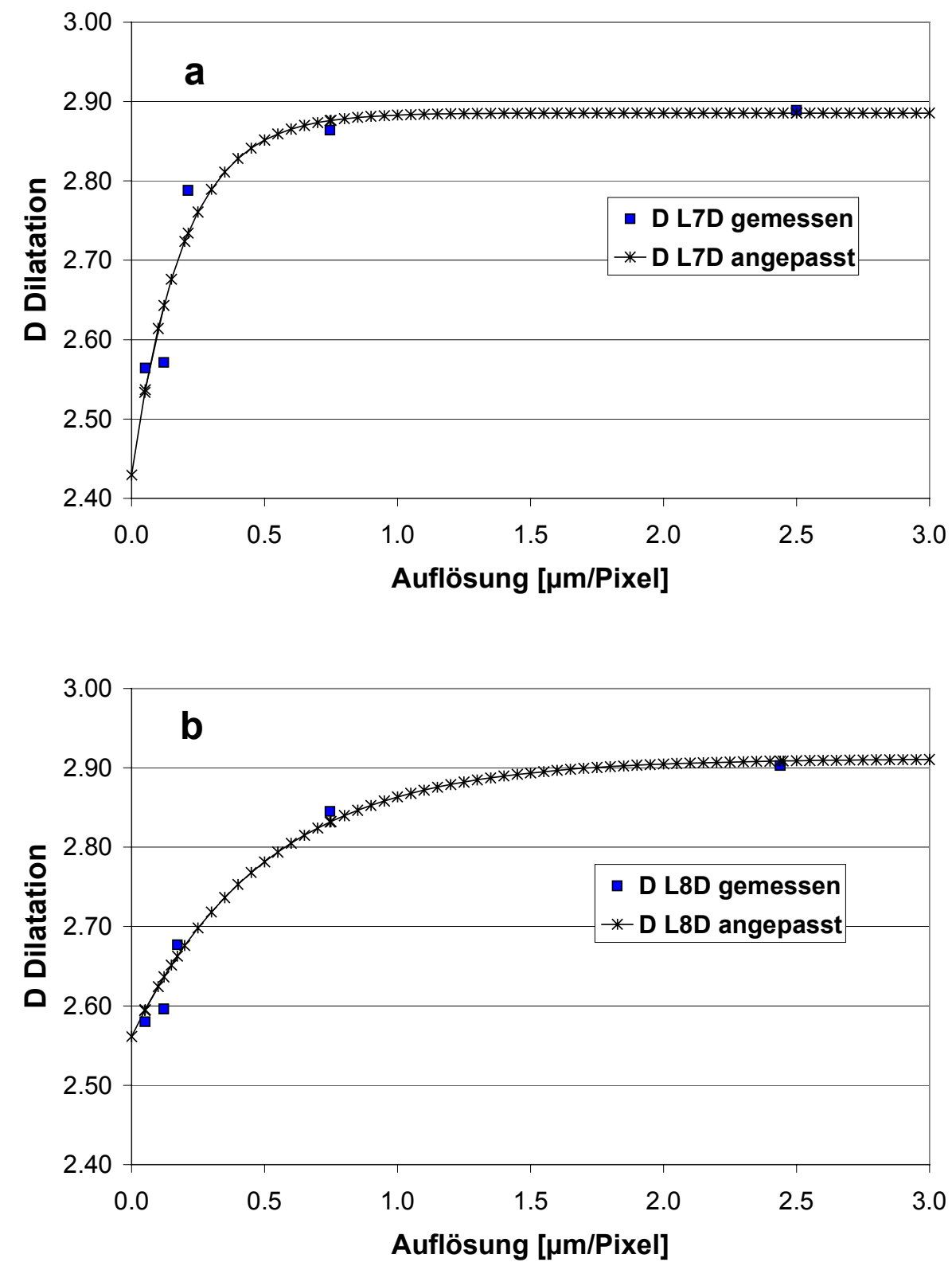

Abb. A.10: Abhängigkeit der gemessenen fraktalen Dimension von der Auflösung, mit der die REM-Bilder aufgenommen wurden. a) Graphische Darstellung für Leo7, Dilatation und b) für Leo8, Dilatation. Die Anpassungsparameter der Gl. 6.1 (Kapitel 6.2) sind in Tab. 6.14 zusammengestellt. 

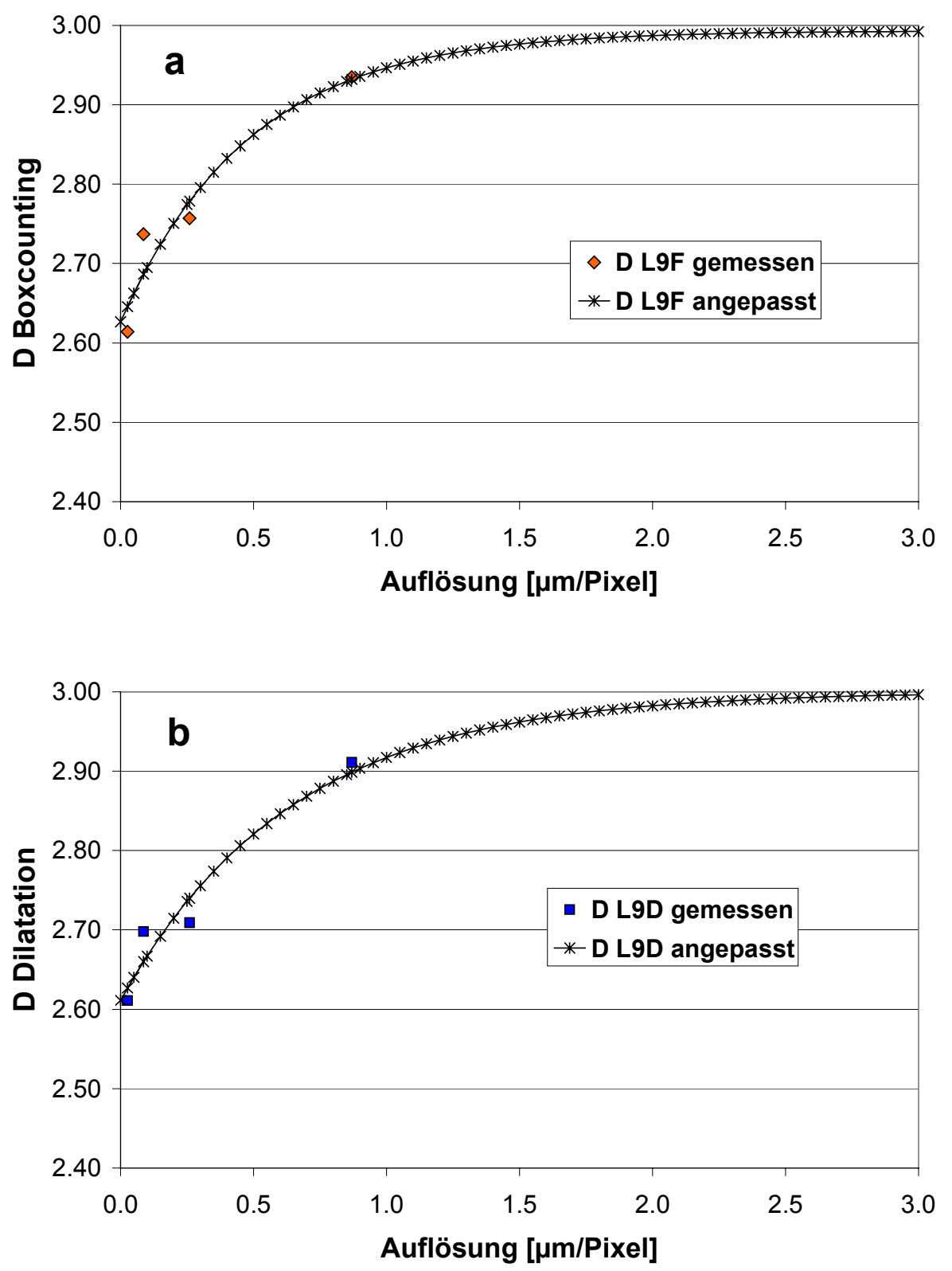

Abb. A.11: Abhängigkeit der gemessenen fraktalen Dimension von der Auflösung, mit der die REM-Bilder aufgenommen wurden. a) Graphische Darstellung für Leo9, Boxcounting und b) für Leo9, Dilatation. Die Anpassungsparameter der Gl. 6.1 (Kapitel 6.2) sind in Tab. 6.14 zusammengestellt. 

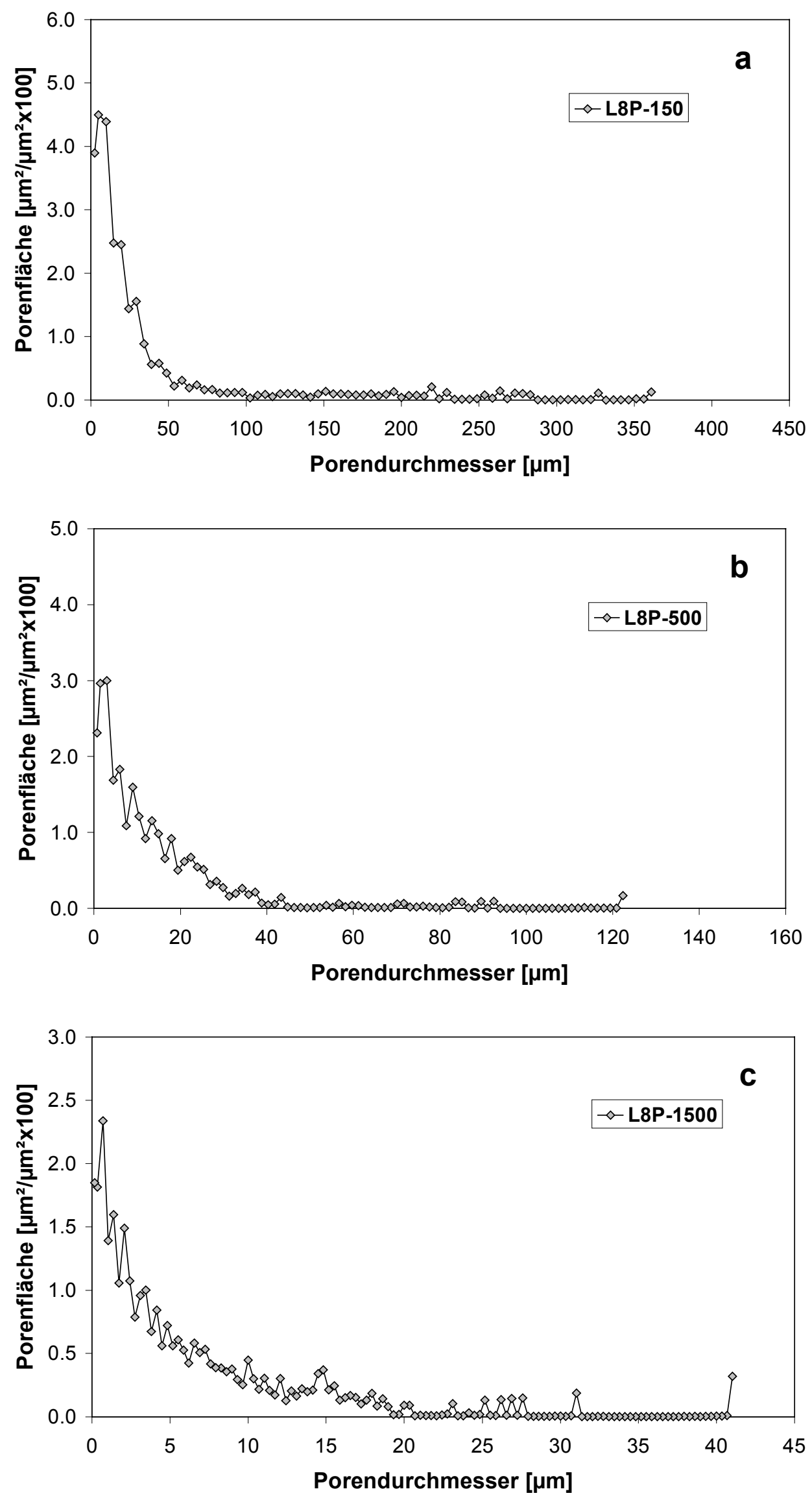

Abb. A.12: Distributive Porengrößenverteilung Serie Leo8. Dargestellt sind die Mittelwerte der Porenflächenhäufigkeiten von je 19 bis 21 Bildern pro Auflösungsstufe und Durchmesser des skalierenden Elementes. Angaben zu den Datenbereichen sind in Tab. A.2 aufgelistet. 

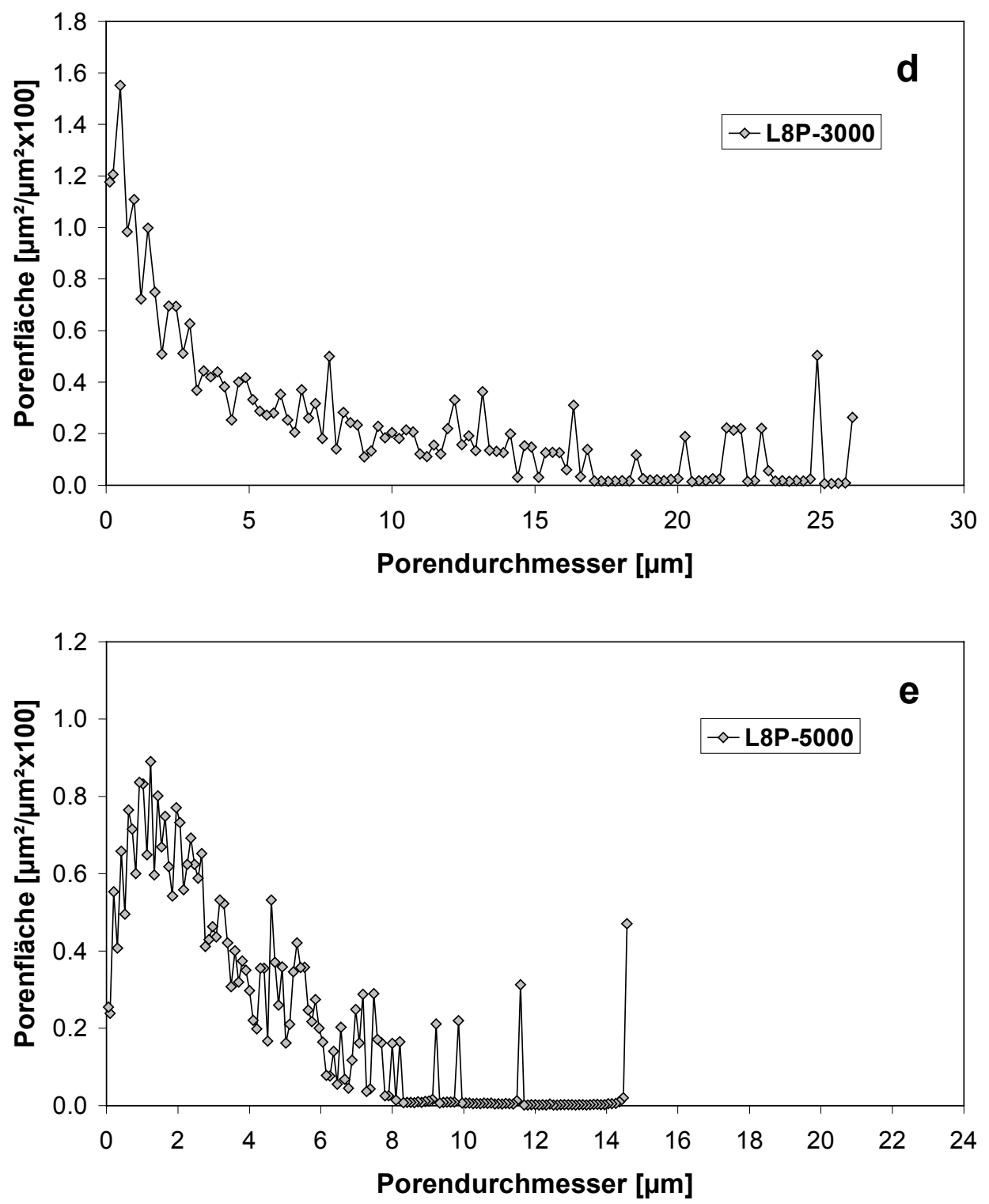

Abb. A.12: Distributive Porengrößenverteilung Serie Leo8. Dargestellt sind die Mittelwerte der Porenflächenhäufigkeiten von je 19 bis 21 Bildern pro Auflösungsstufe und Durchmesser des skalierenden Elementes. Angaben zu den Datenbereichen sind in Tab. A.2 aufgelistet. 

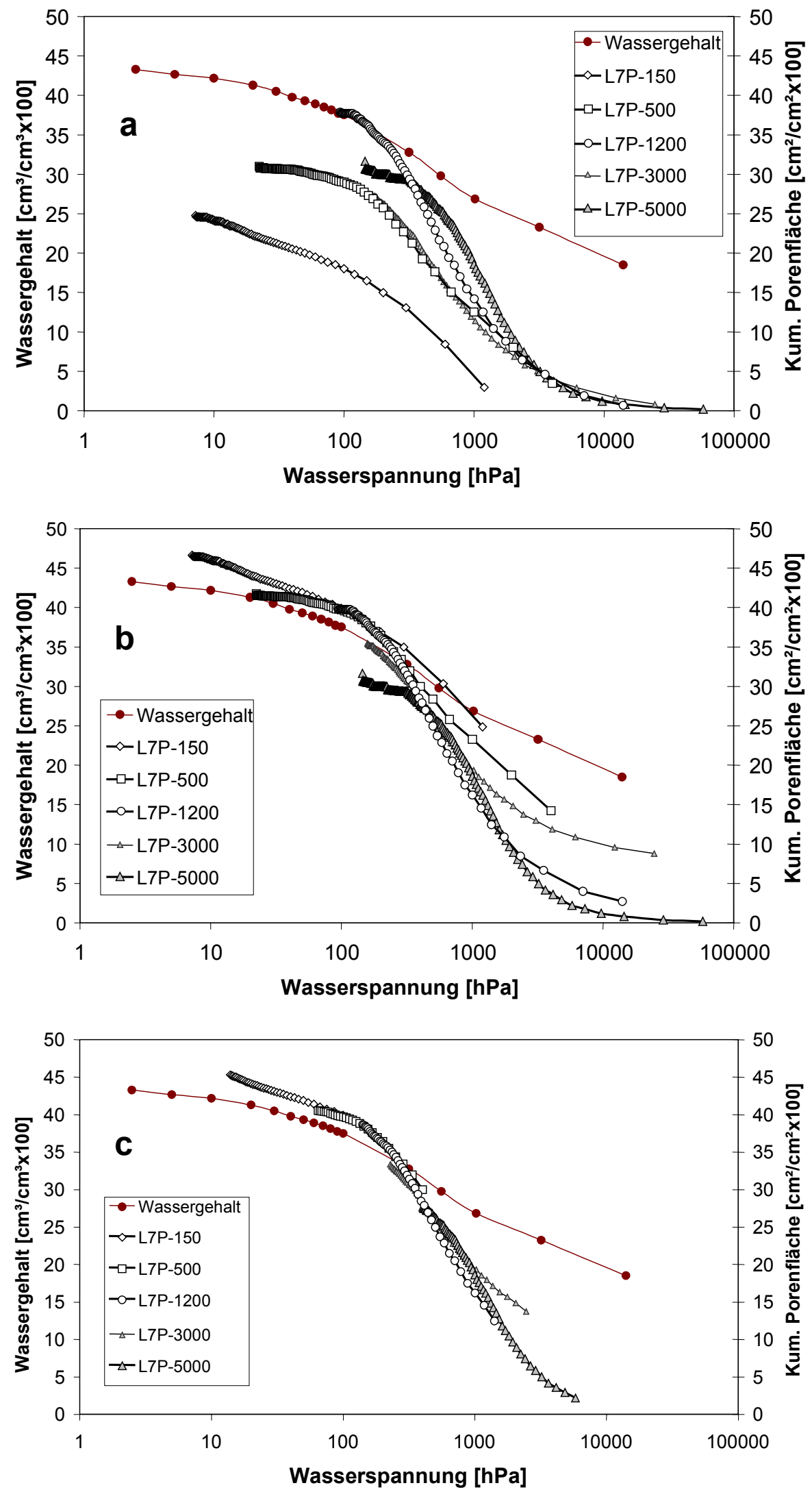

Abb. A.13: Vergleich der Porengrößenverteilungen für Leo7 mit der am selben Bodenhorizont aufgenommenen Retentionskurve. (a) Einzeln dargestellt erscheinen die Kurven parallel verschoben; (b) Aufsummierung und Darstellung aller Werte; (c) Darstellung ohne die ersten fünf Messwerte für die jeweils kleinsten Poren bis zu der Größenklasse, die auf mindestens $50 \%$ aller Bilder vorkommt. 


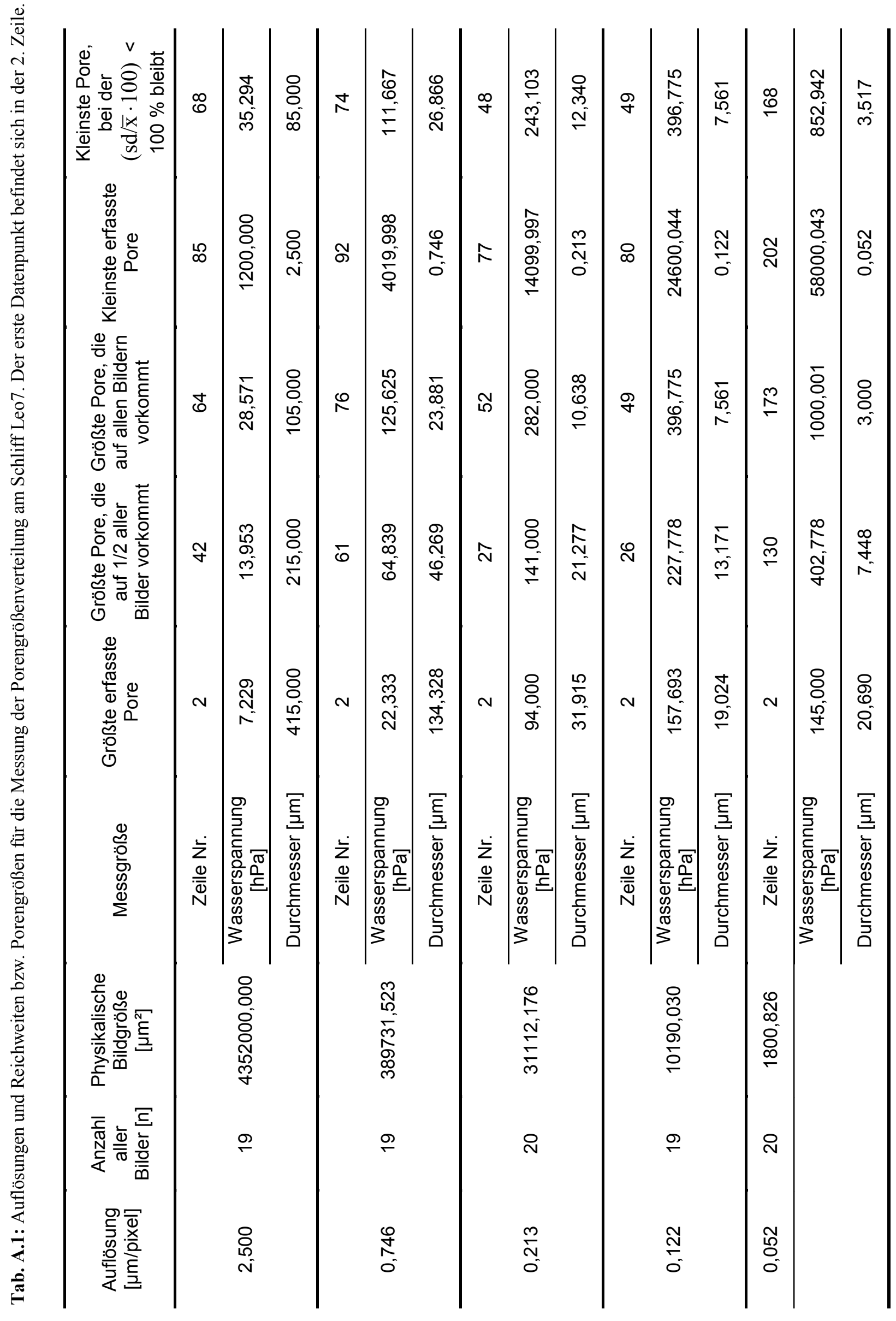





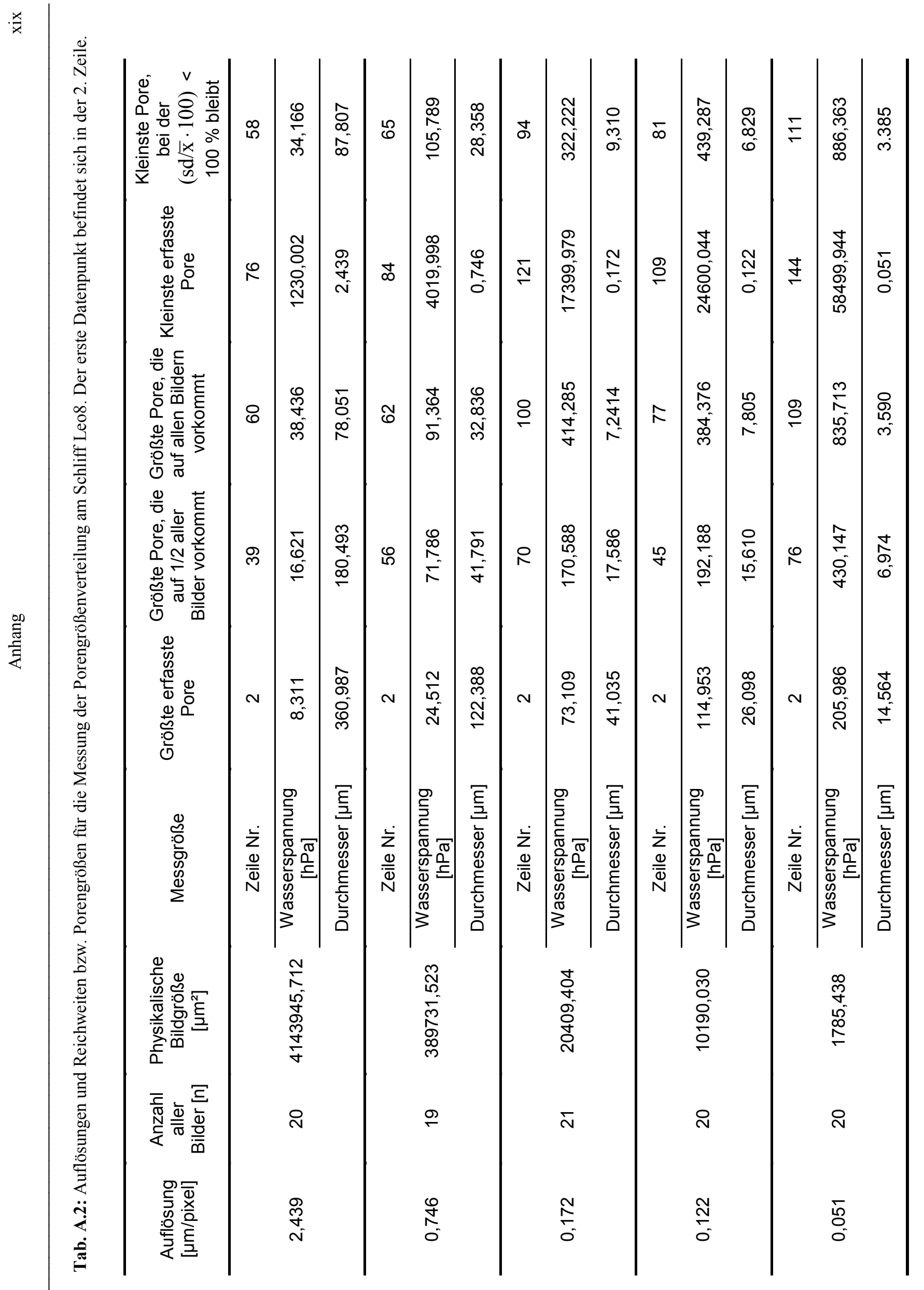





\section{Lebenslauf}

\section{Persönliche Daten}

\section{Schulausbildung}

$$
\begin{array}{r}
1967-1968 \\
1968-1971 \\
1971-1977 \\
1986-1989
\end{array}
$$

\section{Studium}

$01.10 .1989-30.09 .1996$

25.09.1996

$01.04 .1997-30.09 .2001$

27.06.2001

\section{Berufstätigkeit}

$01.10 .1992-31.03 .1993$

$01.10 .1996-31.12 .1996$

$01.03 .1997-31.12 .2001$
Annette Dathe, geb. 18.03.1961 in Braunschweig deutsch geschieden, 2 Kinder Lena Dathe, geb. 1983 Kolja Löblich, geb. 1988

Grundschule in Ganderkesee

Grundschule Heidberg-Süd in Braunschweig Gymnasium Raabeschule in Braunschweig Braunschweig-Kolleg

Erlangung der allgemeinen Hochschulreife

Diplomstudiengang Geoökologie mit den Nebenfächern Ökologie und Ökologische Chemie, Technische Universität Carolo-Wilhelmina zu Braunschweig

Abschluss als Diplom-Geoökologin

Promotionsstudium Geographie, Georg-August-Universität Göttingen

Abschluss des Promotionsverfahrens

Studentische Hilfskraft an der Technischen Universität Carolo-Wilhelmina zu Braunschweig Wissenschaftliche Hilfskraft an der Technischen Universität Carolo-Wilhelmina zu Braunschweig Wissenschaftliche Angestellte an der Georg-August-Universität Göttingen 